\title{
PRECIPITATION DATA FOR WATER YEARS 1992 AND 1993 FROM A NETWORK OF NONRECORDING GAGES AT YUCCA MOUNTAIN, NEVADA
}

\section{U.S. GEOLOGICAL SURVEY}

Open-File Report 95-146

Prepared in cooperation with the NEVADA OPERATIONS OFFICE, U.S. DEPARTMENT OF ENERGY under Interagency Agreement DE-AI08-92NV10874

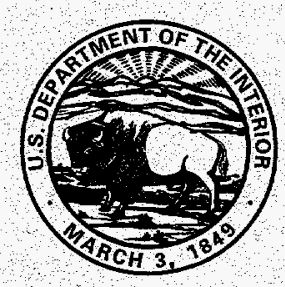




\section{USGS-OFR-- 95-146}

\section{PRECIPITATION DATA FOR WATER YEARS 1992 AND 1993 FROM A NETWORK OF NONRECORDING GAGES AT YUCCA MOUNTAIN, NEVADA}

by Dale S. Ambos, Science Applications International Corporation; Alan L. Flint and Joseph A. Hevesi, U.S. Geological Survey

U.S. GEOLOGICAL SURVEY

Open-File Report 95-146

Prepared in cooperation with the

NEVADA OPERATIONS OFFICE,

U.S. DEPARTMENT OF ENERGY under

Interagency Agreement DE-Al08-92NV10874

Denver, Colorado

1995 


\section{U.S. DEPARTMENT OF THE INTERIOR BRUCE BABBITT, Secretary}

U.S. GEOLOGICAL SURVEY

Gordon P. Eaton, Director

The use of trade, product, industry, or firm names is for descriptive purposes only and does not imply endorsement by the U.S. Government.

For additional information write to: Chief, Earth Science Investigations Program

Yucca Mountain Project Branch U.S. Geological Survey Box 25046, MS 421 Denver Federal Center Denver, CO 80225
Copies of this report can be purchased from: U.S. Geological Survey Earth Science Information Center Open-File Reports Section Box 25286, MS 517 Denver Federal Center Denver, CO 80225 


\section{DISCLAIMER}

Portions of this document may be illegible in electronic image products. Images are produced from the best available original document. 


\section{CONTENTS}

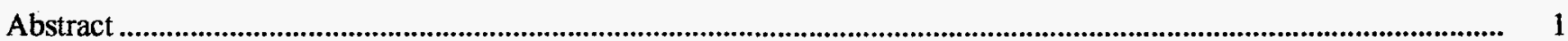

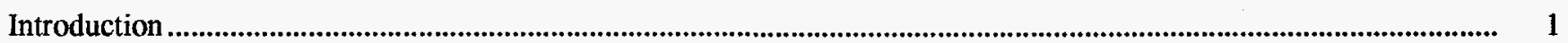

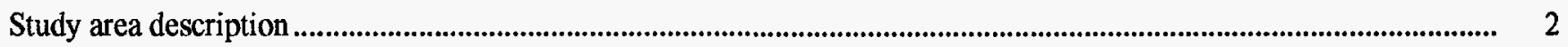

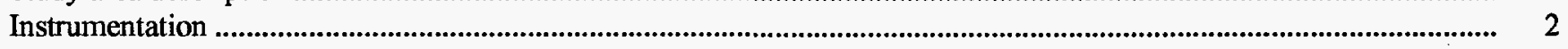

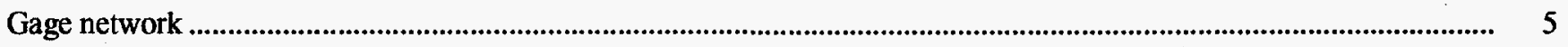

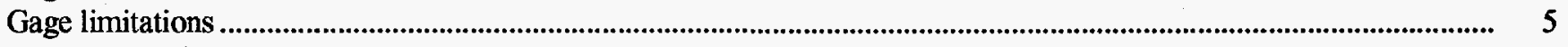

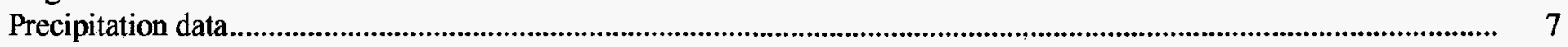

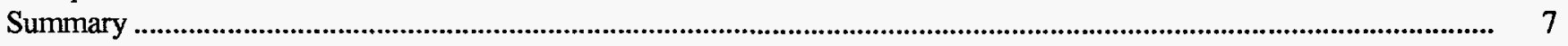

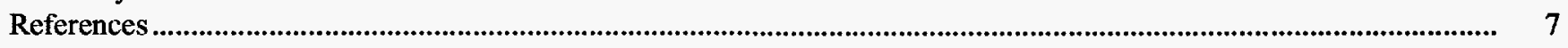

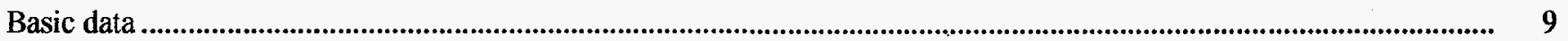

\section{FIGURES}

1. The four primary study area boundaries for regional and site meteorology .......................................................... 3

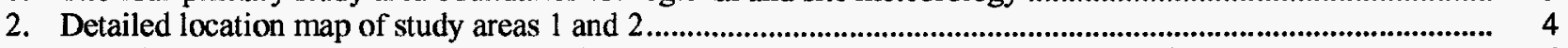

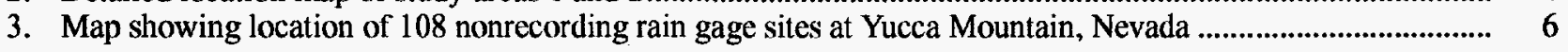

\section{TABLES}

1. U.S. Geological Survey nonrecording precipitation gage network at Yucca Mountain, Nevada

2. Summary of precipitation data from the nonrecording gage network at Yucca Mountain, Nevada, for water years 1992 and 1993.

3. Network precipitation totals for water year 1992

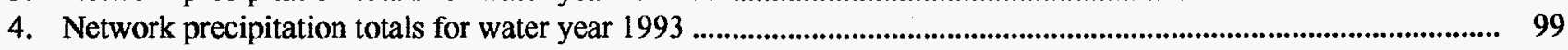

\section{CONVERSION FACTORS}

\begin{tabular}{llll}
\hline Multiply & By & To obtain \\
\hline & inch & 25.4 & millimeter (mm) \\
foot & 0.3048 & meter (m) \\
mile & 1.61 & kilometer (km) \\
\hline
\end{tabular}

Sea level: In this report "sea level" refers to the National Geodetic Vertical Datum of 1929 (NGVD of 1929) - a geodetic datum derived from a general adjustment of the first-order level nets of both the United States and Canada, formerly called Sea Level Datum of 1929.

\section{DISCLAIMER}

This report was prepared as an account of work sponsored by an agency of the United States Government. Neither the United States Government nor any agency thereof, nor any of their employees, makes any warranty, express or implied, or assumes any legal liability or responsibility for the accuracy, completeness, or usefulness of any information, apparatus, product, or process disclosed, or represents that its use would not infringe privately owned rights. Reference herein to any specific commercial product, process, or service by trade name, trademark, manufacturer, or otherwise does not necessarily constitute or imply its endorsement, recommendation, or favoring by the United States Government or any agency thereof. The views and opinions of authors expressed herein do not necessarily state or reflect those of the United States Government or any agency thereof. 


\title{
Precipitation Data for Water Years 1992 and 1993 from a Network of Nonrecording Gages at Yucca Mountain, Nevada
}

\author{
By Dale S. Ambos, Alan L. Flint, and Joseph A. Hevesi
}

\begin{abstract}
This report presents precipitation data collected in a storage gage network at Yucca Mountain, Nevada, from October 1, 1991, to

September 30, 1993. The measured values indicate total accumulated precipitation for specified time intervals approximately corresponding to separate storm events.

Installation of a precipitation monitoring network was initiated in January 1990, and was continually expanded and upgraded throughout the period ending in September 1993. The final network included 3 different gage types for a total of 133 gages at 108 locations within the three drainages overlying the potential repository site. Measured precipitation indicated above average accumulations for water years 1992 and 1993 relative to the most recent estimate of 6.7 inches for long-term average annual precipitation over the area of the network. The total precipitation averaged over the network in 1992 was about 8.2 inches with a maximum of about 11.2 inches measured at borehole USW GA-1. The total precipitation averaged over the network in 1993 was about 10.3 inches with a maximum of about 12.1 inches at neutron-access borehole UE-25 UZN \#4.
\end{abstract}

\section{INTRODUCTION}

Yucca Mountain, Nevada, is a north-south trending block-faulted ridge located within the southern portion of the Basin and Range physiographic province and within the northern extent of the Mojave Desert, and is being evaluated as a potential site for the storage of high-level radioactive waste in a geologic repository (U.S. Department of Energy, 1988). As part of this study, the U.S. Geological Survey (USGS) is collecting precipitation data. Precipitation is the major source of infiltration to the unsaturated zone, and a potential source of recharge for the saturated zone. Precipitation is also the source of all runoff, which is intermittent under the current arid climatic conditions at Yucca Mountain. Precipitation data are needed to help characterize present-day net infiltration rates, to develop 3-dimensional models for predicting changes in infiltration rates in response to potential changes in climate and surface characteristics, and to help predict the hydrologic response of the surface environment to extreme precipitation events which can occur over long periods of time.

To accurately characterize the spatial distribution of precipitation in arid, mountainous terrain, and to study patterns of storm-cell development, movement, and size, dense sampling networks are important (Jones, 1981). For example, a dense sampling network of 100 to 150 monitoring sites covering the area overlying and also directly adjacent to the potential repository site was considered necessary to satisfy the data requirements for detailed 3-dimensional site-scale unsaturated flow modeling. Preliminary studies using available regional data indicated that this density was needed to meet accuracy requirements of approximately 10-percent error for areal estimations and for the characterization of the spatial variability of precipitation. An initial network consisting of 13 automated tipping-bucket precipitation gages (both heated and nonheated) was installed at Yucca Mountain in 1987. However, limited resources prevented the installation of a sufficiently dense network using this relatively expensive instrumentation.

To satisfy the density requirement for the 3-dimensional model, a network of nonrecording or storage-type gages was installed beginning in 1990 to supplement the tipping-bucket gages. Although storage gages do not record the timing and intensity of precipitation, they can provide an accurate measurement of accumulated precipitation. A primary disadvantage of the 3 different types of storage gages used in the network is that measurements must be manually obtained from each gage; total precipitation depth following a precipitation event is recorded by visual observation of a fluid level relative to a calibration mark. Due to the 
number of gages involved and the area of coverage, daily readings of the network were not practical. Instead, an effort was made to read the entire network immediately following a period of relatively continuous precipitation, referred to in this report as a "precipitation event". Specifically, precipitation events were defined by periods of continuous daily precipitation separated by one or more dry days. However, it was not always possible to record total precipitation depth at each gage for each individual event, especially when events were separated by only one dry day. In such cases, measured precipitation amounts represented accumulations resulting from "storm periods", which were more generally defined as periods of two or more sequential precipitation events. Storm periods were interpreted as precipitation amounts resulting from a single synoptic-scale upper-level weather system. Measurements obtained from the tipping-bucket gage network were used to indicate the timing and duration of precipitation events, and also to identify separate events within storm periods.

This report presents precipitation data as they were recorded following each significant precipitation event or storm period. The data will directly support the water balance studies being conducted at 98 borehole sites, geostatistical analysis of the spatial variability of precipitation for isohyetal mapping and stochastic simulation, and for the analysis of the influence of topography and site exposure on precipitation measurements at Yucca Mountain. For example,

Hevesi and others (1994) presented the results of a geostatistical analysis using these data to investigate differences in the spatial variability of precipitation events relative to seasonal influences on storm genesis. Future studies have been planned to use these data for developing and calibrating distributed rainfall-runoff models needed to predict the influence of extreme precipitation events, extended periods of precipitation, changing climatic conditions, and changing surface conditions on infiltration and the redistribution of moisture at Yucca Mountain.

\section{STUDY AREA DESCRIPTION}

Hydrologic studies relevant to site characterization at Yucca Mountain area are being conducted for various different study areas having different scales and levels of need in terms of the accuracy and detail of precipitation measurements. Study area 1 is the smallest, but most critical site in terms of hydrologic characterization, and includes the primary physiographic features overlying the potential repository and all closely adjacent areas. These features consist of the general north-south trending ridge that forms Yucca Mountain, and the three major surface drainages over- lying the potential repository; Drillhole Wash drainage, Busted Butte drainage, and Solitario Canyon drainage. Study area 1 is the most extensively monitored in terms of collecting precipitation data. Precipitation gages also are located in study area 2, the Upper Fortymile Wash drainage, which is important because of its potential contribution to regional ground-water flow and recharge to the saturated zone. Most of the gages that compose the network discussed in this report are located in area 1; a few gages are located in areas 2 and 3 to the northeast and east of area 1 . In general, precipitation data collected for studies conducted in areas 3 and 4 are obtained using a regional network of cooperative stations operated by various agencies, such as the National Oceanic and Atmospheric Administration, the Bureau of Land Management, and the State of Nevada's Desert Research Institute, and are not presented in this report. The boundaries and location of the study areas are shown in figures 1 and 2.

\section{INSTRUMENTATION}

The initial type of gage used in the network was an inexpensive wedge-shaped plastic gage with a 2.5 -inch by 2.75 -inch rectangular opening. The gages have the scale imprinted on the front side in inches and the reverse side in millimeters. Measurements are recorded by visually inspecting the fluid level relative to the calibrated scale. Measurement resolution is 0.01 inches for accumulations less than 0.2 inches, 0.02 inches for accumulations between 0.2 and 1.00 inches, and 0.05 inches for accumulations greater than 1.00 inch.

A second type of gage used is a plastic canister with a circular orifice diameter of 4 inches and a funnel that directs water into an internal measuring tube with a capacity of 1 inch and a measurement resolution of 0.01 inches. Measurements are again recorded by a visual inspection of the fluid level. If precipitation is in excess of 1 inch, the measuring tube overflows into the canister. The total accumulation can be measured by accounting for the contents of the inner tube, emptying the tube, then transferring the overflow water in the canister into the inner tube to be measured and repeating the process as necessary until the canister is emptied. The readings are combined into a total catch.

The third type of nonrecording gage used is a metal canister with a circular 8-inch diameter orifice, that is the standard of the National Weather Service. Precipitation accumulations are measured by inserting a dipstick and observing the fluid mark along a graduated scale having a resolution of 0.01 inches.

For all three gage types, a small amount of mineral oil is used inside the gage to minimize evaporation, and a screen is used inside the orifice to minimize con- 


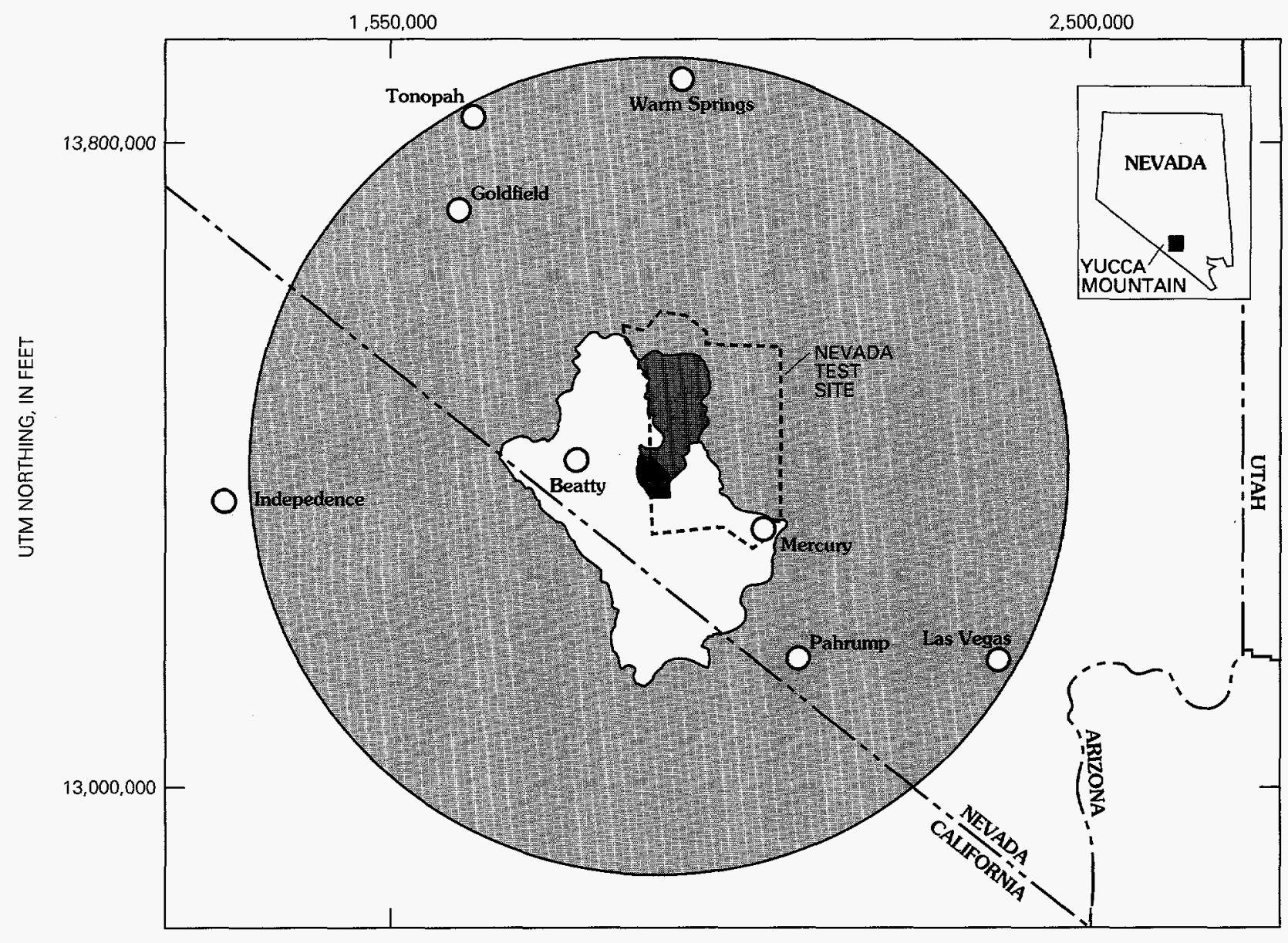

EXPLANATION

Study Area 1-Yucca Mountain

Study Area 2-Upper Fortymile Canyon watershed

Study Area 3-Upper Amargosa River watershed

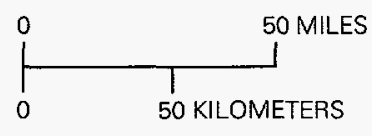

Study Area 4-150 km Radius

Figure 1. The four primary study area boundaries for regional and site meteorology. 


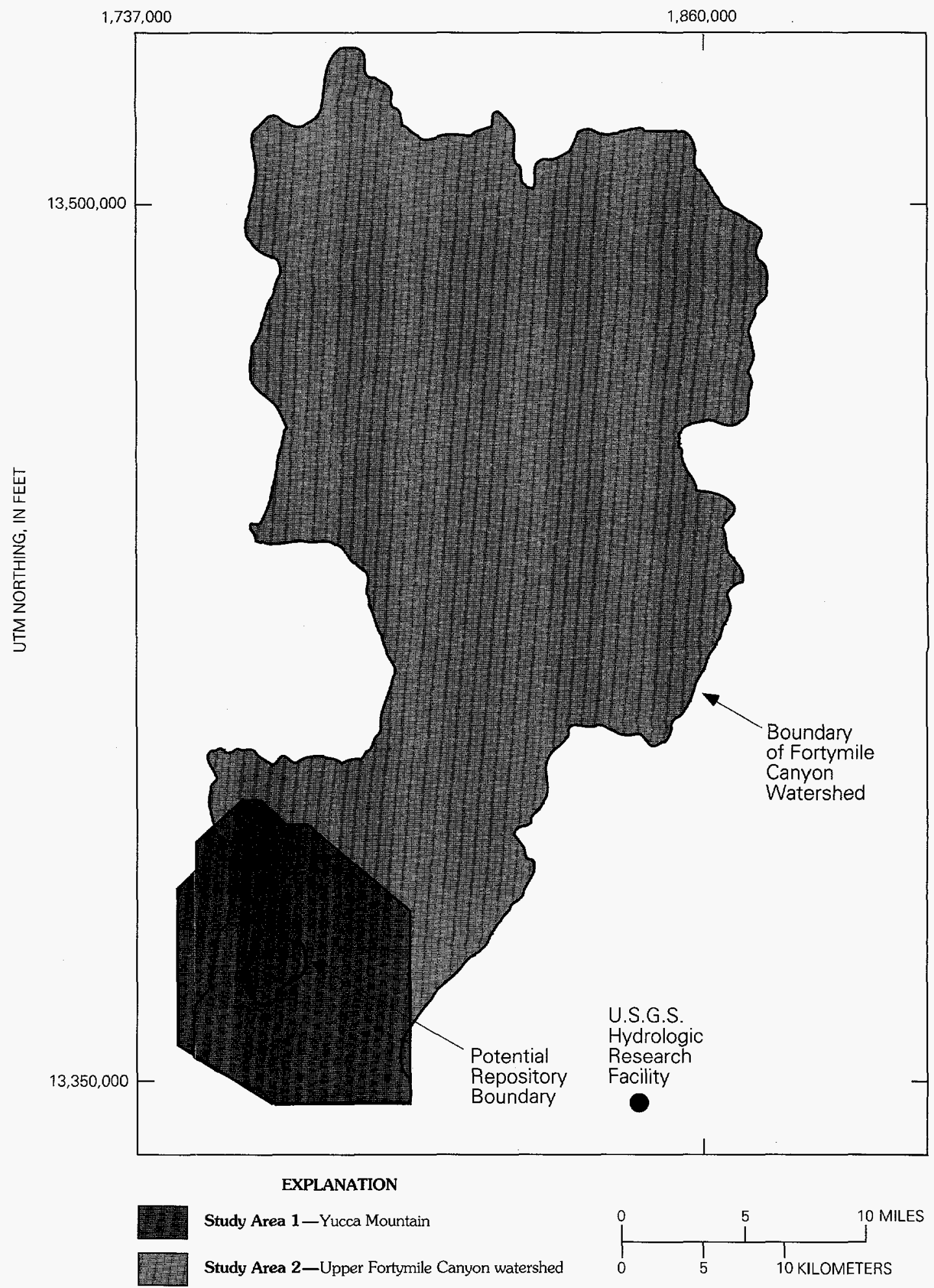

Figure 2. Detailed location map of study areas 1 and 2. 
tamination from debris and wildlife. For the plastic wedges, antifreeze is also used under the oil to mix with collected precipitation to minimize freezing. Locations and elevations for all gages are listed in table 1 , and the different gage types are identified. It should be noted that table 1 indicates the network that existed on September 30,1993, and that the installation date refers to the date which the field site first became operational, and does not necessarily indicate the date which the gage type indicated became operational. For example, the initial network in January 1990 consisted entirely of the plastic wedge-type gage. The network was then continually upgraded at later dates by replacing the wedges with the more accurate 4-inch canisters and 8 -inch metal gages.

\section{GAGE NETWORK}

Before January 1990, the USGS storage-gage network at Yucca Mountain consisted of 14 plastic wedge-type rain gages. The gages were located at streamflow-measurement sites and provided some information on rainfall quantities in specific subdrainages for the purpose of studying intermittent runoff events and for water balance studies. Beginning in January 1990, the network was expanded to a total of 93 plastic wedge gages. Eighty five gages were mounted on 2 -inch by 4 -inch posts, which were attached to the exposed casing of vertical boreholes used in monitoring soil water content by logging with hand-held neutron-moisture meters. The gages were installed at these locations because precipitation measurements were needed to help analyze observed changes in the water content profiles. The remaining gages were mounted on steel fenceposts and installed at proposed borehole sites and also sites where the spatial coverage of boreholes was sparse. Gages were installed at heights of 4 to 5 feet above ground surface at all locations to prevent splash-in and to help minimize contamination.

During 1991, the network was expanded to 96 sites, and retrofitting of the plastic wedge gages with the 4-inch canister gages was initiated. At some sites, the wedge gage was not replaced but was supplemented with the canister gage for the purpose of comparing catch measurements. During 1992, the network was again expanded to a total of 108 sites. Retrofitting continued, and the 8-inch metal canister-type gage was added to the network. The present nonrecording gage network consists of 108 sites and 133 gages. The use of multiple gages at certain locations is continuing for the purpose of identifying and quantifying systematic differences in measurements between the three nonrecording gage types. The location of the nonrecording storage-gage network at the time of September 30, 1993, is indicated in figure 3.

\section{GAGE LIMITATIONS}

Analysis of measurements obtained at sites where both the 4 -inch and the 8-inch canister gages were located consistently indicated good agreement to within 0.01 inches. At sites where both the wedge-type gage and the 4-inch canisters gage were located, comparisons indicated that the wedge-type gage readings were consistently 10 to 15 percent less than readings from the 4-inch canisters when measuring liquid precipitation. The catch deficit is attributed to an increase in the influence of wind turbulence above the orifice of the wedge gage because of a combination of orifice shape and size. In the case of measuring precipitation occurring as snow, measurement deficit problems due to wind turbulence are much greater, and additional problems are encountered in the use of storage-type gages for measuring frozen precipitation amounts. Some modifications to the gages were made during winter season operation in an effort to mitigate these problems. This included the removal of all screens in gages because insect contamination was at a minimum, and the screens accumulated frozen precipitation which could be blown out of the gage by wind. For the 4 -inch canister gage, the funnel top does not allow much snow to accumulate before overflowing or getting blown out. The wedge provides a deeper cavity in which to collect snowfall, and therefore, tended to measure higher precipitation amounts than the 4-inch canister during snowfall events.

The circular gages can be set up for collecting snow by removing the top funnel and the measuring tube. The canister will trap snowfall which then is melted and measured using the measuring tube. This technique was tested during the winter of 1992-93, without oil and antifreeze, but met with limited success. The disadvantage of this technique is that the gage must be attended to as soon after the storm as possible to prevent sublimation of the snow or evaporation of the melt. On average, measurement of snowfall depth was estimated to be only $50-60$ percent of the true snowfall depth using the storage gages. Additional tests are currently being conducted to include oil and antifreeze when setting up to measure snow catch along the crest of Yucca Mountain (average elevation 4,900 feet). In general, it should be noted that the wedge-type and 4-inch canister-type gages were not specifically intended by the manufacturer to be used for measuring snowfall amounts; measurements were often taken after the snow collected in the gage had melted. During the 1992 to 1993 winter season (water year 1992), some relatively cold periods occurred 


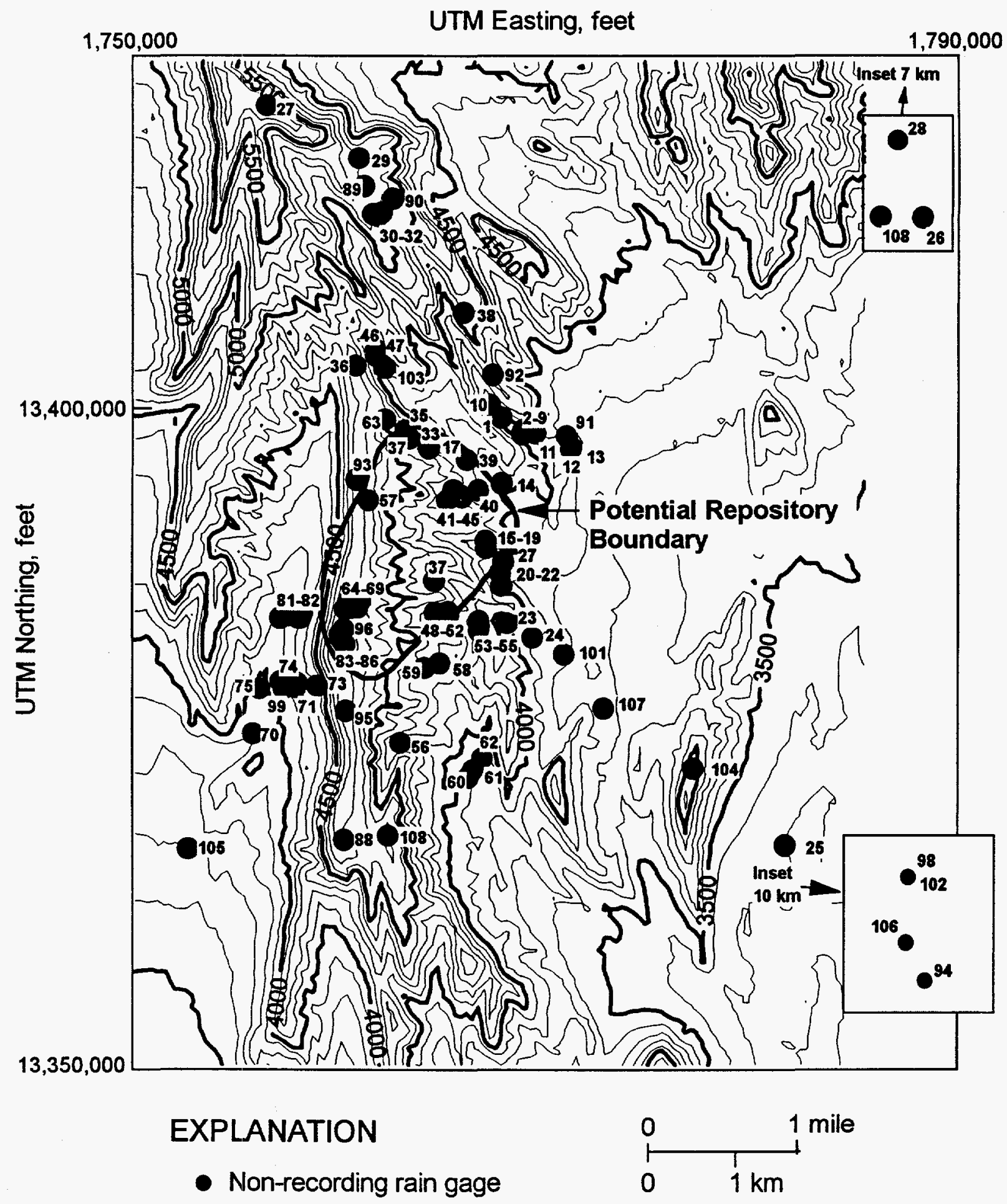

Figure 3. Map showing location of 108 nonrecording rain gage sites at Yucca Mountain, Nevada. 
when the collected snowfall did not melt soon after the precipitation event, and an attempt was made to measure snow density which could then be used for calculating an equivalent liquid precipitation depth. Snow samples were taken, and the measured water-to-snow density ratio ranged from 7 for relatively dry snow to 3.5 for relatively wet snow. In all cases, the presence of frozen precipitation in storage gages was carefully noted in the record. Studies investigating the comparison of measurements obtained using the propaneheated tipping-bucket precipitation gages and the storage gage network are continuing.

\section{PRECIPITATION DATA}

The precipitation data for the period October 1, 1991, through September 30, 1993 (water years 1992 and 1993), are presented in table 2. The table is organized to show separate storm events for the dates indicated. There were 42 significant storm events over the 2-year period, 21 in each of the water years. Although every attempt was made to gather the data after each storm event, the network was not always read after each event due to the large number of gages, the abnormally high frequency of storms, and the remote location of many of the gages. When readings were missed, the record includes two or more days of precipitation; however, the dates of each event are included in the table (the total catch for the specific gage is still valid). Total precipitation at each gage with a complete annual record for water years 1992 and 1993 are listed in tables 3 and 4, respectively.

\section{SUMMARY}

Precipitation data were collected during water years 1992 and 1993 using a network of 93 to 133 nonrecording, storage-type gages located at 108 monitoring sites on Yucca Mountain. The network consists of three different types of gages and was established initially to supplement the areal coverage of the automated tipping-bucket gage network and to provide sitespecific data for infiltration and water balance studies being conducted at borehole locations. The average total precipitation for 84 continuously read gages in water year 1992 was about 8.2 inches, with a maximum total of about 11.2 inches recorded at borehole USW GA-1 (map code: 89, figure 3). For water year 1993, the average total precipitation for 100 continuously read gages was about 10.3 inches, with a maximum total precipitation of about 12.1 inches recorded at UE-25 USN \#4 (map code: 4, figure 3).

Problems concerning catch deficit at more exposed sites, particularly at higher elevation sites where snowfall is more likely, were identified, and studies are continuing in an effort to improve measurement accuracy.

\section{REFERENCES}

Hevesi, J.A., Ambos, D.S., and Flint, A.L., 1994, A preliminary characterization of the spatial variability of precipitation at Yucca Mountain, Nevada: Proceedings, High Level Radioactive Waste Management Meeting, Las Vegas, Nevada.

Jones, K.R., 1981, Arid zone hydrology for agricultural development: Rome, Food and Agriculture Organization of the United Nations, FAO Irrigation and Drainage Paper No. 37.

Nichols, W.D., 1987, Geohydrology of the unsaturated zone at the burial site for low-level radioactive waste near Beatty, Nye County, Nevada: U.S. Geological Survey Water-Supply Paper 2312, $57 \mathrm{p}$.

U.S. Department of Energy, 1988, Yucca Mountain Site Characterization Plan. DOE/RW-0199, U.S. Department of Energy, Office of Civilian Radioactive Waste Management Office, Washington, D.C. 

BASIC DATA 
Table 1. U.S. Geological Survey nonrecording precipitation gage network at Yucca Mountain, Nevada

[*, installation date of first gage at multiple gage sites: 2, wedge gage; 4 and 8, 4-inch and 8-inch canisters, respectively; Northing and Easting are in Universal Transverse Mercator coordinates]

\begin{tabular}{|c|c|c|c|c|c|c|}
\hline $\begin{array}{l}\text { Location } \\
\text { code }\end{array}$ & $\begin{array}{c}\text { Site } \\
\text { nomenclature }\end{array}$ & Gage type & $\begin{array}{l}\text { Northing } \\
\text { (feet) }\end{array}$ & $\begin{array}{c}\text { Easting } \\
\text { (feet) }\end{array}$ & $\begin{array}{l}\text { Altitude } \\
\text { (feet) }\end{array}$ & Date installed ${ }^{*}$ \\
\hline 1 & UE-25 UZN \#1 & 4 & $13,384,730$ & $1,803,107$ & 3,995 & $1 / 26 / 90$ \\
\hline 2 & UE-25 UZN \#2 & 4 & $13,384,010$ & $1,803,999$ & 3,947 & $1 / 23 / 90$ \\
\hline 3 & UE-25 UZN \#3 & 2 & $13,384,034$ & $1,804,004$ & 3,941 & $1 / 23 / 90$ \\
\hline 4 & UE-25 UZN \#4 & 4 & $13,384,067$ & $1,804,012$ & 3,942 & $1 / 08 / 90$ \\
\hline 5 & UE-25 UZN \#5 & 2 & $13,384,093$ & $1,804,019$ & 3,943 & $1 / 10 / 90$ \\
\hline 6 & UE-25 UZN \#6 & 2 & $13,384,110$ & $1,804,022$ & 3,938 & $1 / 10 / 90$ \\
\hline 7 & UE-25 UZN \#7 & 4 & $13,384,128$ & $1,804,026$ & 3,939 & $1 / 08 / 90$ \\
\hline 8 & UE-25 UZN \#8 & 2 & $13,384,147$ & $1,804,031$ & 3,939 & $1 / 08 / 90$ \\
\hline 9 & UE-25 UZN \#9 & 4 & $13,384,186$ & $1,804,040$ & 3,941 & $1 / 08 / 90$ \\
\hline 10 & UE-25 UZN \#10 & 4 & $13,385,268$ & $1,802,625$ & 4,038 & $1 / 08 / 90$ \\
\hline 11 & UE-25 UZN \#12 & 2 & $13,384,057$ & $1,804,580$ & 3,907 & $1 / 23 / 90$ \\
\hline 12 & UE-25 UZN $\# 13$ & 4 & $13,383,437$ & $1,806,141$ & 3,821 & $1 / 23 / 90$ \\
\hline 13 & UE-25 UZN $\$ 14$ & 2 & $13,383,379$ & $1,806,120$ & 3,824 & $1 / 23 / 90$ \\
\hline 14 & UE-25 UZN ; & 2 & $13,381,874$ & $1,803,140$ & 4,019 & $1 / 30 / 90$ \\
\hline 15 & UE-25 UZN $: 119$ & 4 & $13,379,089$ & $1,802,474$ & 4,025 & $1 / 05 / 90$ \\
\hline 16 & UE-25 UZN 洋20 & 4 & $13,379,160$ & $1,802,481$ & 4,027 & $1 / 24 / 90$ \\
\hline 17 & UE-25 UZN 洋21 & $2 / 4$ & $13,379,206$ & $1,802,493$ & 4,028 & $1 / 05 / 90$ \\
\hline 18 & UE-25 UZN 样22 & 4 & $13,379,280$ & $1,802,507$ & 4,029 & $1 / 05 / 90$ \\
\hline 19 & UE-25 UZN \#23 & 4 & $13,379,373$ & $1,802,447$ & 4,043 & $1 / 05 / 90$ \\
\hline 20 & UE-25 UZN \#28 & 2 & $13,378,494$ & $1,803,224$ & 3,958 & $1 / 24 / 90$ \\
\hline 21 & UE-25 UZN \#29 & 4 & $13,378,015$ & $1,803,079$ & 3,973 & $1 / 05 / 90$ \\
\hline 22 & UE-25 UZN \#30 & 4 & $13,377,451$ & $1,803,141$ & 3,959 & $1 / 24 / 90$ \\
\hline 23 & UE-25 UZN \#56 & 4 & $13,375,798$ & $1,803,394$ & 3,960 & $1 / 24 / 90$ \\
\hline 24 & UE-25 UZN \#60 & 4 & $13,375,165$ & $1,804,483$ & 3,892 & $1 / 24 / 90$ \\
\hline 25 & UE-25 UZN \#85 & 4 & $13,366,165$ & $1,815,512$ & 3,337 & $1 / 05 / 90$ \\
\hline 26 & UE-25 UZN \#+92 & $2 / 4$ & $13,393,472$ & $1,821,406$ & 3,669 & $1 / 30 / 90$ \\
\hline 27 & UE-25 UZN 非7 & 4 & $13,378,497$ & $1,803,225$ & 3,958 & $1 / 05 / 90$ \\
\hline 28 & UE-29 UZN \#91 & $2 / 4$ & $13,412,738$ & $1,823,121$ & 3,647 & $1 / 30 / 90$ \\
\hline 29 & USW UZ-N11 & $2 / 4$ & $13,395,950$ & $1,796,866$ & 5,224 & $4 / 13 / 92$ \\
\hline 30 & USW UZ-N15 & 4 & $13,393,470$ & $1,797,706$ & 5,109 & $4 / 13 / 92$ \\
\hline 31 & USW UZ-N16 & 4 & $13,393,530$ & $1,797,479$ & 5,117 & $4 / 13 / 92$ \\
\hline 32 & USW UZ-N17 & 4 & $13,393,604$ & $1,797,848$ & 5,128 & $4 / 13 / 92$ \\
\hline 33 & USW UZ-N24 & $2 / 4$ & $13,383,395$ & $1,799,942$ & 4,227 & $1 / 03 / 90$ \\
\hline 34 & USW UZZ-N25 & 4 & $13,383,817$ & $1,799,106$ & 4,335 & $1 / 03 / 90$ \\
\hline 35 & USW UZ-N26 & 4 & $13,384,143$ & $1,798,909$ & 4,384 & $1 / 03 / 90$ \\
\hline
\end{tabular}


Table 1. U.S. Geological Survey nonrecording precipitation gage network at Yucca Mountain, Nevada--Continued

\begin{tabular}{|c|c|c|c|c|c|c|}
\hline $\begin{array}{l}\text { Location } \\
\text { code }\end{array}$ & $\begin{array}{c}\text { Site } \\
\text { nomenclature }\end{array}$ & Gage type & $\begin{array}{c}\text { Northing } \\
\text { (feet) }\end{array}$ & $\begin{array}{l}\text { Easting } \\
\text { (feet) }\end{array}$ & $\begin{array}{l}\text { Altitude } \\
\text { (feet) }\end{array}$ & Date installed* \\
\hline 36 & USW UZ-N27 & $2 / 8$ & $13,386,948$ & $1,796,749$ & 4,859 & $5 / 09 / 90$ \\
\hline 37 & USW UZ-N37 & 8 & $13,377,656$ & $1,800,218$ & 4,247 & $11 / 06 / 92$ \\
\hline 38 & USW UZ-N36 & 4 & $13,389,294$ & $1,801,450$ & 4,642 & $4 / 13 / 92$ \\
\hline 39 & USW UZ-N37 & $2 / 4$ & $13,382,895$ & $1,801,604$ & 4,124 & 9/23/91 \\
\hline 40 & USW UZ-N40 & $2 / 4$ & $13,381,574$ & $1,802,115$ & 4,079 & $1 / 08 / 90$ \\
\hline 41 & USW UZ-N41 & 4 & $13,381,263$ & $1,801,416$ & 4,118 & $1 / 10 / 90$ \\
\hline 42 & USW UZ-N42 & 4 & $13,381,122$ & $1,800,755$ & 4,179 & $1 / 08 / 90$ \\
\hline 43 & USW UZ-N43 & $2 / 4$ & $13,381,392$ & $1,801,159$ & 4,149 & $1 / 08 / 90$ \\
\hline 44 & USW UZ-N44 & 4 & $13,381,587$ & $1,801,034$ & 4,162 & $1 / 08 / 90$ \\
\hline 45 & USW UZ-N45 & 4 & $13,381,372$ & $1,801,324$ & 4,130 & $1 / 08 / 90$ \\
\hline 46 & USW UZ-N46 & 4 & $13,387,643$ & $1,797,622$ & 4,501 & $1 / 03 / 90$ \\
\hline 47 & USW UZ-N47 & 2 & $13,387,349$ & $1,797,659$ & 4,480 & $1 / 03 / 90$ \\
\hline 48 & USW UZ-N48 & 4 & $13,376,228$ & $1,800,327$ & 4,211 & $1 / 05 / 90$ \\
\hline 49 & USW UZ-N49 & 4 & $13,376,253$ & $1,800,235$ & 4,229 & $1 / 25 / 90$ \\
\hline 50 & USW UZ-N50 & $2 / 4$ & $13,376,171$ & $1,800,825$ & 4,173 & $1 / 05 / 90$ \\
\hline 51 & USW UZ-N51 & 4 & $13,376,256$ & $1,800,822$ & 4,169 & $1 / 25 / 90$ \\
\hline 52 & USW UZ-N52 & 2 & $13,376,289$ & $1,800,822$ & 4,172 & $1 / 25 / 90$ \\
\hline 53 & USW UZ-N53 & 2 & $13,375,496$ & $1,802,152$ & 4,056 & $9 / 26 / 91$ \\
\hline 54 & USW UZ-N54 & $2 / 4$ & $13,375,672$ & $1,802,177$ & 4,046 & $9 / 26 / 91$ \\
\hline 55 & USW UZ-N55 & 4 & $13,375,903$ & $1,802,162$ & 4,073 & $9 / 26 / 91$ \\
\hline 56 & USW UZ-N57 & 4 & $13,370,553$ & $1,798,763$ & 4,186 & $6 / 21 / 90$ \\
\hline 57 & USW UZ-N64 & 8 & $13,381,109$ & $1,797,333$ & 4,791 & $7 / 22 / 92$ \\
\hline 58 & USW UZ-N65 & 2 & $13,374,021$ & $1,800,458$ & 4,372 & $1 / 30 / 90$ \\
\hline 59 & USW UZ-N66 & 4 & $13,373,826$ & $1,799,803$ & 4,356 & $1 / 02 / 90$ \\
\hline 60 & USW UZ-N67 & $2 / 4$ & $13,369,034$ & $1,801,737$ & 3,920 & $1 / 30 / 90$ \\
\hline 61 & USW UZ-N68 & 2 & $13,369,363$ & $1,801,943$ & 3,925 & $1 / 30 / 90$ \\
\hline 62 & USW UZ-N69 & 4 & $13,369,863$ & $1,802,337$ & 3,918 & $1 / 25 / 90$ \\
\hline 63 & USW UZ-N70 & $2 / 4$ & $13,384,634$ & $1,798,049$ & 4,542 & $1 / 03 / 90$ \\
\hline 64 & USW UZ-N71 & $2 / 4$ & $13,376,405$ & $1,796,320$ & 4,925 & $1 / 02 / 90$ \\
\hline 65 & USW UZ-N72 & 2 & $13,376,448$ & $1,796,539$ & 4,889 & $1 / 31 / 90$ \\
\hline 66 & USW UZ-N73 & 2 & $13,376,430$ & $1,796,839$ & 4,867 & $1 / 31 / 90$ \\
\hline 67 & USW UZ-N74 & 2 & $13,376,742$ & $1,796,472$ & 4,904 & $1 / 02 / 90$ \\
\hline 68 & USW UZ-N75 & $2 / 4$ & $13,376,843$ & $1,796,988$ & 4,799 & $1 / 31 / 90$ \\
\hline 69 & USW UZ-N76 & 2 & $13,376,734$ & $1,796,960$ & 4,958 & $1 / 31 / 90$ \\
\hline 70 & USW UZ-N77 & 2 & $13,370,893$ & $1,792,331$ & 3,901 & $1 / 18 / 90$ \\
\hline
\end{tabular}


Table 1. U.S. Geological Survey nonrecording precipitation gage network at Yucca Mountain, Nevada--Continued

\begin{tabular}{|c|c|c|c|c|c|c|}
\hline $\begin{array}{l}\text { Location } \\
\text { code }\end{array}$ & $\begin{array}{c}\text { Site } \\
\text { nomenclature }\end{array}$ & Gage type & $\begin{array}{l}\text { Northing } \\
\text { (feet) }\end{array}$ & $\begin{array}{c}\text { Easting } \\
\text { (feet) }\end{array}$ & $\begin{array}{l}\text { Altitude } \\
\text { (feet) }\end{array}$ & Date installed* \\
\hline 71 & USW UZ-N78 & 2 & $13,372,931$ & $1,794,188$ & 4,182 & $1 / 14 / 90$ \\
\hline 72 & USW UZ-N79 & 2 & $13,373,106$ & $1,794,260$ & 4,155 & $1 / 14 / 90$ \\
\hline 73 & USW UZ-N80 & $2 / 4$ & $13,373,010$ & $1,795,127$ & 4,332 & $1 / 14 / 90$ \\
\hline 74 & USW UZ-N81 & 2 & $13,373,177$ & $1,793,521$ & 4,065 & $1 / 14 / 90$ \\
\hline 75 & USW UZ-N82 & 2 & $13,372,865$ & $1,792,617$ & 3,975 & $1 / 18 / 90$ \\
\hline 76 & USW UZ-N83 & 2 & $13,375,996$ & $1,794,265$ & 4,157 & $1 / 14 / 90$ \\
\hline 77 & USW UZ-N84 & 2 & $13,376,087$ & $1,793,803$ & 4,112 & $1 / 14 / 90$ \\
\hline 78 & USW UZ-N86 & 2 & $13,375,987$ & $1,794,376$ & 4,172 & $1 / 14 / 90$ \\
\hline 79 & USW UZ-N87 & 2 & $13,376,084$ & $1,793,802$ & 4,112 & $1 / 14 / 90$ \\
\hline 80 & USW UZ-N88 & 4 & $13,376,170$ & $1,794,466$ & 4,202 & $1 / 14 / 90$ \\
\hline 81 & USW UZ-N89 & 2 & $13,375,979$ & $1,793,505$ & 4,090 & $1 / 14 / 90$ \\
\hline 82 & USW UZ-N90 & $2 / 4$ & $13,375,977$ & $1,793,503$ & 4,090 & $1 / 14 / 90$ \\
\hline 83 & USW UZ-N93 & 2 & $13,374,963$ & $1,796,240$ & 4,924 & $1 / 30 / 90$ \\
\hline 84 & USW UZ-N94 & 2 & $13,375,103$ & $1,796,154$ & 4,926 & $1 / 02 / 90$ \\
\hline 85 & USW UZ-N95 & 2 & $13,375,278$ & $1,796,090$ & 4,929 & $1 / 30 / 90$ \\
\hline 86 & USW UZ-N96 & 2 & $13,374,826$ & $1,796,322$ & 4,893 & $1 / 02 / 90$ \\
\hline 87 & USW UZ-N98 & $2 / 4$ & $13,383,386$ & $1,799,972$ & 4,223 & $1 / 03 / 90$ \\
\hline 88 & USW UZ 13 & 2 & $13,366,320$ & $1,796,308$ & 4,820 & $1 / 02 / 90$ \\
\hline 89 & USW GA-1 & 4 & $13,394,742$ & $1,797,096$ & 5,187 & $2 / 05 / 90$ \\
\hline 90 & USW G-2 & 4 & $13,394,206$ & $1,798,355$ & 5,098 & $2 / 05 / 90$ \\
\hline 91 & UE25 WT-4 & 2 & $13,383,923$ & $1,805,925$ & 3,835 & $2 / 05 / 90$ \\
\hline 92 & UE25 WT-18 & 4 & $13,386,566$ & $1,802,731$ & 4,383 & $2 / 05 / 90$ \\
\hline 93 & USW H-5 & 2 & $13,382,013$ & $1,796,803$ & 4,851 & $2 / 05 / 90$ \\
\hline 94 & HRF & & $2 / 4 / 8$ & $13,354,458$ & $1,849,156$ & 3,410 \\
\hline 95 & USW H-3 & 2 & $13,371,923$ & $1,796,381$ & 4,866 & $5 / 09 / 90$ \\
\hline 96 & WX 4 (old) & 2 & $13,375,513$ & $1,796,273$ & 4,915 & $5 / 11 / 90$ \\
\hline 97 & WX 5 (Prow) & 2 & $13,398,211$ & $1,792,824$ & 5,870 & $7 / 10 / 90$ \\
\hline 98 & Evaporation pan & 2 & $13,359,634$ & $1,848,522$ & 3,460 & $8 / 06 / 90$ \\
\hline 99 & WX 4 (new) & $4 / 8$ & $13,372,871$ & $1,793,666$ & 4,900 & $10 / 01 / 90$ \\
\hline 100 & G-3 & & $2 / 8$ & $13,366,524$ & $1,798,213$ & 4,765 \\
\hline 101 & WX 1 & & $4 / 8$ & $13,374,424$ & $1,805,852$ & 3,815 \\
\hline 102 & WX 2 & & 8 & $13,359,534$ & 1,848523 & 3,492 \\
\hline 103 & wX 3 & & $2 / 8$ & $13,386,864$ & $1,798,250$ & 4,432 \\
\hline 104 & Fran Ridge & $2 / 8$ & $13,369,449$ & $1,811,509$ & 4,062 & $10 / 05 / 90$ \\
\hline 105 & Plug Hill & 2 & $13,365,910$ & $1,789,505$ & 3,710 & $11 / 07 / 90$ \\
\hline 106 & $4 \mathrm{JA}$ & & 8 & $13,356,407$ & $1,848,575$ & 3,422 \\
\hline 107 & Knothead Gap & 8 & $13,372,079$ & $1,807,596$ & 3,720 & $11 / 06 / 92$ \\
\hline 108 & Upper Fortymile & 8 & $13,393,491$ & $1,819,567$ & 3,765 & $11 / 06 / 92$ \\
\hline
\end{tabular}


Table 2. Summary of precipitation data from the nonrecording gage network at Yucca Mountain, Nevada, for water years 1992 and 1993

[*, Includes storm of September 28, 1991; $\mathrm{M}$, missing data; $\mathrm{M}^{*}$, missing from this storm but included in subsequent reading(s); **, includes precipitation from all previous storms indicated by $\mathrm{M}^{*}$;

NI, gage not installed; EVAP, evaporation possible or likely to have occurred; SNOW, precipitation fell as snow and reading may be low; T, trace (less than 0.005 in.); FZN, precipitation in gage is frozen; @, plastic wedge gage replaced with 4-in. diameter canister gage prior to measurement]

\begin{tabular}{|c|c|c|c|c|c|c|c|c|c|c|c|c|c|c|}
\hline $\begin{array}{l}\text { Loca- } \\
\text { tlon } \\
\text { code. }\end{array}$ & $\begin{array}{c}\text { Site } \\
\text { nomenclature }\end{array}$ & $\begin{array}{l}\text { Amount } \\
\text { (inches) }\end{array}$ & $\begin{array}{l}\text { Date } \\
\text { read }\end{array}$ & Notes & $\begin{array}{l}\text { Loca- } \\
\text { tion } \\
\text { code }\end{array}$ & $\begin{array}{c}\text { Site } \\
\text { nomenclature }\end{array}$ & $\begin{array}{l}\text { Amount } \\
\text { (inches) }\end{array}$ & $\begin{array}{l}\text { Date } \\
\text { read }\end{array}$ & Notes & $\begin{array}{l}\text { Loca- } \\
\text { tion } \\
\text { code }\end{array}$ & $\begin{array}{c}\text { Site } \\
\text { nomenclature }\end{array}$ & $\begin{array}{l}\text { Amount } \\
\text { (Inches) }\end{array}$ & $\begin{array}{l}\text { Date } \\
\text { read }\end{array}$ & Notes \\
\hline \multicolumn{15}{|c|}{ STORM OF OCTOBER 26, 1991} \\
\hline 1 & UE-25 UZN \#1 & 0 & $10 / 28$ & & 39 & USW UZ-N37 & 0 & $10 / 28$ & & 74 & USW UZ-N81 & .07 & $11 / 6$ & * \\
\hline 2 & UE-25 UZN \#2 & 0 & $10 / 28$ & & 39 & N37 4" Gage & NI & & & 75 & USW UZ-N82 & .11 & $11 / 6$ & * \\
\hline 3 & UE-25 UZN \#3 & 0 & $10 / 28$ & & 40 & USW UZ-N40 & 0 & $10 / 28$ & & 75 & N82 4" Gage & $\mathrm{NI}$ & & \\
\hline 4 & UE-25 UZN \#4 & 0 & $10 / 28$ & & 40 & N40 4" Gage & NI & & & 76 & USW UZ-N83 & .13 & $11 / 6$ & $*$ \\
\hline 4 & N4 4" Gage & $\mathrm{NI}$ & & & 41 & USW UZ-N4I & 0 & $10 / 28$ & & 77 & USW UZ-N84 & .10 & $11 / 6$ & $*$ \\
\hline 5 & UE-25 UZN \#5 & 0 & $10 / 28$ & & 42 & USW UZ-N42 & 0 & $10 / 28$ & & 78 & USW UZ-N86 & .13 & $11 / 6$ & $*$ \\
\hline 6 & UE-25 UZN \#6 & 0 & $10 / 28$ & & 43 & USW UZ-N43 & 0 & $10 / 28$ & & 79 & USW UZ-N87 & .07 & $11 / 6$ & $*$ \\
\hline 7 & UE-25 UZN \#7 & 0 & $10 / 28$ & & 43 & N43 4" Gage & NI & & & 80 & USW UZ-N88 & .10 & $11 / 6$ & $*$ \\
\hline 8 & UE-25 UZN \#8 & 0 & $10 / 28$ & & 44 & USW UZ-N44 & $\mathbf{M}$ & & & 81 & USW UZ-N89 & .11 & $11 / 6$ & $*$ \\
\hline 9 & UE-25 UZN \#9 & 0 & $10 / 28$ & & 45 & USW UZ-N45 & 0 & $10 / 28$ & & 82 & USW UZ-N90 & .08 & $11 / 6$ & $*$ \\
\hline 10 & UE-25 UZN \#10 & 0 & $10 / 28$ & & 46 & USW UZ-N46 & .06 & $10 / 28$ & & 82 & N90 4" Gage & NI & & \\
\hline 10 & N10 4" Gage & NI & & & 47 & USW UZ-N47 & .05 & $10 / 28$ & & 83 & USW UZ-N93 & 0 & $10 / 28$ & \\
\hline 11 & UE-25 UZN \#12 & 0 & $10 / 28$ & & 48 & USW UZ-N48 & .01 & $10 / 28$ & & 84 & USW UZ-N94 & 0 & $10 / 28$ & \\
\hline 12 & UE-25 UZN \#13 & 0 & $10 / 28$ & & 49 & USW UZ-N49 & 0 & $10 / 28$ & & 85 & USW UZ-N95 & 0 & $10 / 28$ & \\
\hline 13 & UE-25 UZN \#14 & $\mathbf{M}$ & & & 50 & USW UZ-N50 & 0 & $10 / 28$ & & 86 & USW UZ-N96 & 0 & $10 / 28$ & \\
\hline 14 & UE-25 UZN \#18 & $\mathbf{M}$ & & & 50 & N50 4" Gage & NI & & & 87 & USW UZ-N98 & 0 & $10 / 28$ & \\
\hline 15 & UE-25 UZN \#19 & 0 & $10 / 28$ & & 51 & USW UZ-N51 & 0 & $10 / 28$ & & 87 & N98 4" Gage & NI & & \\
\hline 16 & UE-25 UZN \#20 & 0 & $10 / 28$ & & 52 & USW UZ-N52 & 0 & $10 / 28$ & & 88 & USW UZ-13 & 0 & $10 / 28$ & \\
\hline 17 & UE-25 UZN \#21 & 0 & $10 / 28$ & & 53 & USW UZ-N53 & .25 & $10 / 28$ & & 88 & UZ-13 4" Gage & NI & & \\
\hline 17 & N21 4" Gage & NI & & & 54 & USW UZ-N54 & 0 & $10 / 28$ & & 89 & USW GA-1 & .04 & $10 / 28$ & \\
\hline 18 & UE-25 UZN \#22 & 0 & $10 / 28$ & & 54 & N54 4" Gage & NI & & & 90 & USW G-2 & .03 & $10 / 28$ & \\
\hline 19 & UE-25 UZN \#23 & 0 & $10 / 28$ & & 55 & USW UZ-N55 & 0 & $10 / 28$ & & 91 & UE-25 WT-4 & .01 & $10 / 28$ & \\
\hline 20 & UE-25 UZN \#28 & .02 & $10 / 28$ & & 56 & USW UZ-N57 & 0 & $10 / 28$ & & 92 & UE-25 WT-18 & .01 & $10 / 28$ & \\
\hline 21 & UE-25 UZN \#29 & .01 & $10 / 28$ & & 57 & USW UZ-N64 & NI & & & 93 & USW H-5 & 0 & $10 / 28$ & \\
\hline 22 & UE-25 UZN \#30 & .01 & $10 / 28$ & & 58 & USW UZ-N65 & 0 & $10 / 28$ & & 94 & HRF Wedge & .17 & $10 / 28$ & \\
\hline 23 & UE-25 UZN \#56 & .03 & $10 / 28$ & & 59 & USW UZ-N66 & 0 & $10 / 28$ & & 94 & HRF 4" Gage & NI & & \\
\hline 24 & UE-25 UZN \#60 & .03 & $10 / 28$ & & 60 & USW UZ-N67 & .01 & $10 / 28$ & & 94 & HRF 8" Gage & NI & & \\
\hline 25 & UE-25 UZN \#85 & .21 & $10 / 28$ & & 60 & N67 4" Gage & $\mathrm{NI}$ & & & 95 & USW H-3 & .01 & $10 / 28$ & \\
\hline 26 & UE-25 UZN \#92 & .09 & $10 / 28$ & & 61 & USW UZ-N68 & .01 & $10 / 28$ & & 96 & Weather station 4 (old) & 0 & $10 / 28$ & \\
\hline 26 & N92 4" Gage & NI & & & 62 & USW UZ-N69 & $\mathbf{M}$ & & & 97 & Weather station 5 (Prow) & $\mathbf{M}$ & & \\
\hline
\end{tabular}




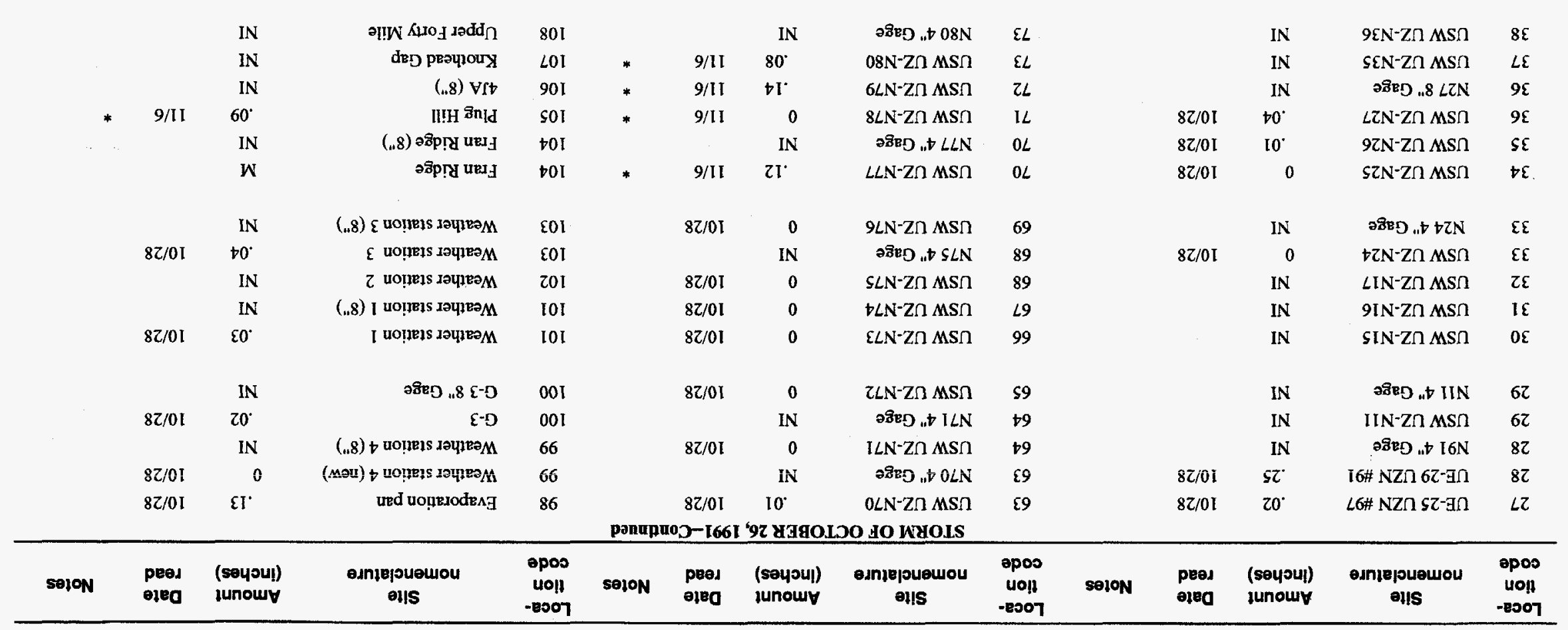


Table 2. Summary of precipitation data from the nonrecording gage network at Yucca Mountain, Nevada, for water years 1992 and 1993 --Continued

\begin{tabular}{|c|c|c|c|c|c|c|c|c|c|c|c|c|c|c|}
\hline $\begin{array}{l}\text { Loca- } \\
\text { tion } \\
\text { code }\end{array}$ & $\begin{array}{c}\text { Site } \\
\text { nomenclature }\end{array}$ & $\begin{array}{l}\text { Amount } \\
\text { (Inches) }\end{array}$ & $\begin{array}{l}\text { Date } \\
\text { read }\end{array}$ & Notes & $\begin{array}{l}\text { Loca- } \\
\text { tion } \\
\text { code }\end{array}$ & $\begin{array}{c}\text { Site } \\
\text { nomenclature }\end{array}$ & $\begin{array}{l}\text { Amount } \\
\text { (inches) }\end{array}$ & $\begin{array}{l}\text { Date } \\
\text { read }\end{array}$ & Notes & $\begin{array}{l}\text { Loca- } \\
\text { tion } \\
\text { code }\end{array}$ & $\begin{array}{c}\text { Site } \\
\text { nomenclature }\end{array}$ & $\begin{array}{l}\text { Amount } \\
\text { (inches) }\end{array}$ & $\begin{array}{l}\text { Date } \\
\text { read }\end{array}$ & Notes \\
\hline \multicolumn{15}{|c|}{ STORM OF NOVEMBER 14-15, 1991} \\
\hline 1 & UE-25 UZN \#1 & .01 & $11 / 15$ & & 39 & USW UZ-N37 & 0 & $11 / 15$ & & 74 & USW UZ-N81 & $\mathrm{M}^{*}$ & & \\
\hline 2 & UE- 25 UZN \#2 & $\mathrm{M}$ & $11 / 15$ & & 39 & N37 4" Gage & $\mathrm{NI}$ & $11 / 15$ & & 75 & USW UZ-N82 & $\mathrm{M}^{*}$ & & \\
\hline 3 & UE-25 UZN \#3 & .01 & $11 / 15$ & & 40 & USW UZ-N40 & 0 & & & 75 & N82 4" Gage & NI & & \\
\hline 4 & UE-25 UZN \#4 & .01 & $11 / 15$ & & 40 & N40 4" Gage & NI & $11 / 15$ & & 76 & USW UZ-N83 & $\mathrm{M}^{*}$ & & \\
\hline 4 & N4 4" Gage & NI & & & 41 & USW UZ-N41 & $\mathrm{T}$ & & & 77 & USW UZ-N84 & $\mathbf{M}^{*}$ & & \\
\hline 5 & UE-25 UZN \#5 & $T$ & $11 / 15$ & & 42 & USW UZ-N42 & 0 & $11 / 15$ & & 78 & USW UZ-N86 & $\mathbf{M}^{*}$ & & \\
\hline 6 & UE-25 UZN \#6 & .01 & $11 / 15$ & & 43 & USW UZ-N43 & 0 & $11 / 15$ & & 79 & USW UZ-N87 & $\mathbf{M}^{*}$ & & \\
\hline 7 & UE-25 UZN \#7 & .01 & $11 / 15$ & & 43 & N43 4" Gage & NI & $11 / 15$ & & 80 & USW UZ-N88 & $\mathrm{M}^{*}$ & & \\
\hline 8 & UE-25 UZN \#8 & .01 & $11 / 15$ & & 44 & USW UZ-N44 & 0 & & & 81 & USW UZ-N89 & $\mathbf{M}^{*}$ & & \\
\hline 9 & UE-25 UZN \#9 & 0 & $11 / 15$ & & 45 & USW UZ-N45 & 0 & $11 / 15$ & & 82 & USW UZ-N90 & $\mathrm{M}^{*}$ & & \\
\hline 10 & UE-25 UZN $\# 10$ & 0 & $11 / 15$ & & 46 & USW UZ-N46 & $\mathrm{M}^{*}$ & $11 / 15$ & & 82 & N90 4" Gage & NI & & \\
\hline 10 & N10 4" Gage & $\mathrm{NI}$ & & & 47 & USW UZ-N47 & $\mathrm{M}^{*}$ & & & 83 & USW UZ-N93 & 0 & $11 / 15$ & \\
\hline 11 & UE-25 UZN \#12 & .01 & $11 / 15$ & & 48 & USW UZ-N48 & $\mathrm{M}^{*}$ & & & 84 & USW UZ-N94 & 0 & $11 / 15$ & \\
\hline 12 & UE- 25 UZN $\# 13$ & .01 & $11 / 15$ & & 49 & USW UZ-N49 & $\mathrm{M}^{*}$ & & & 85 & USW UZ-N95 & 0 & $11 / 15$ & \\
\hline 13 & UE-25 UZN \#14 & 0 & $11 / 15$ & & 50 & USW UZ-N50 & $\mathrm{M}^{*}$ & & & 86 & USW UZ-N96 & 0 & $11 / 15$ & \\
\hline 14 & UE-25 UZN \#18 & .01 & $11 / 15$ & & 50 & N50 4" Gage & NI & $11 / 15$ & & 87 & USW UZ-N98 & 0 & $11 / 15$ & \\
\hline 15 & UE-25 UZN \#19 & $\mathrm{M}^{*}$ & & & 51 & USW UZ-N51 & 0 & & & 87 & N98 4" Gage & $\mathrm{NI}$ & & \\
\hline 16 & UE- 25 UZN $\# 20$ & $\mathbf{M}^{*}$ & & & 52 & USW UZ-N52 & 0 & $11 / 15$ & & 88 & USW UZ-13 & .02 & $11 / 15$ & \\
\hline 17 & UE-25 UZN \#21 & $\mathbf{M}^{*}$ & & & 53 & USW UZ-N53 & 0 & $11 / 15$ & & 88 & UZ-13 4" Gage & NI & & \\
\hline 17 & N21 4" Gage & NI & & & 54 & USW UZ-N54 & $\mathbf{M}$ & $11 / 15$ & & 89 & USW GA-1 & .01 & $11 / 15$ & \\
\hline 18 & UE-25 UZN \#22 & $\mathbf{M}^{*}$ & & & 54 & N54 4" Gage & $\mathrm{NI}$ & & & 90 & USW G-2 & .01 & $11 / 15$ & \\
\hline 19 & UE- 25 UZN $\# 23$ & $\mathbf{M}^{*}$ & & & 55 & USW UZ-N55 & 0 & & & 91 & UE-25 WT-4 & 0 & $11 / 15$ & \\
\hline 20 & UE-25 UZN \#28 & $\mathrm{M}^{*}$ & & & 56 & USW UZ-N57 & 0 & $11 / 15$ & & 92 & UE-25 WT-18 & .01 & $11 / 15$ & \\
\hline 21 & UE- 25 UZN $\# 29$ & $\mathrm{M}^{*}$ & & & 57 & USW UZ-N64 & NI & $11 / 15$ & & 93 & USW H-5 & 0 & $11 / 15$ & \\
\hline 22 & UE- 25 UZN $\# 30$ & 0 & $11 / 15$ & & 58 & USW UZ-N65 & 0 & & & 94 & HRF Wedge & .01 & $11 / 15$ & \\
\hline 23 & UE-25 UZN \#56 & 0 & $11 / 15$ & & 59 & USW UZ-N66 & 0 & $11 / 15$ & & 94 & HRF 4" Gage & NI & & \\
\hline 24 & UE-25 UZN $\# 60$ & 0 & $11 / 15$ & & 60 & USW UZ-N67 & 0 & $11 / 15$ & & 94 & HRF 8" Gage & NI & & \\
\hline 25 & UE-25 UZN \#85 & .01 & $11 / 15$ & & 60 & N67 4" Gage & NI & $11 / 15$ & & 95 & USW H-3 & 0 & $11 / 15$ & \\
\hline 26 & UE-25 UZN \#92 & 0 & $11 / 15$ & & 61 & USW UZ-N68 & .01 & & & 96 & Weather station 4 (old) & 0 & $11 / 15$ & \\
\hline 26 & N92 4" Gage & NI & & & 62 & USW UZ-N69 & 0 & $11 / 15$ & & 97 & Weather station 5 (Prow) & $\mathbf{M}$ & & \\
\hline
\end{tabular}


क Table 2. Summary of precipitation data from the nonrecording gage network at Yucca Mountain, Nevada, for water years 1992 and $1993-$ Continued

\begin{tabular}{|c|c|c|c|c|c|c|c|c|c|c|c|c|c|c|}
\hline $\begin{array}{l}\text { Loca- } \\
\text { tion } \\
\text { code }\end{array}$ & $\begin{array}{c}\text { Site } \\
\text { nomenclature }\end{array}$ & $\begin{array}{l}\text { Amount } \\
\text { (inches) }\end{array}$ & $\begin{array}{l}\text { Date } \\
\text { read }\end{array}$ & Notes & $\begin{array}{l}\text { Loca- } \\
\text { tion } \\
\text { code }\end{array}$ & $\begin{array}{c}\text { Site } \\
\text { nomenclature }\end{array}$ & $\begin{array}{l}\text { Amount } \\
\text { (inches) }\end{array}$ & $\begin{array}{l}\text { Date } \\
\text { read }\end{array}$ & Notes & $\begin{array}{l}\text { Loca- } \\
\text { tion } \\
\text { code }\end{array}$ & $\begin{array}{c}\text { Site } \\
\text { nomenclature }\end{array}$ & $\begin{array}{l}\text { Amount } \\
\text { (inches) }\end{array}$ & $\begin{array}{l}\text { Date } \\
\text { read }\end{array}$ & Notes \\
\hline \multicolumn{15}{|c|}{ STORM OF NOVEMBER 14-15, 1991-Continued } \\
\hline 27 & UE-25 UZN \#97 & $\mathbf{M}^{*}$ & & & 63 & USW UZ-N70 & 0 & $11 / 15$ & & 98 & Evaporation pan & .06 & $11 / 15$ & \\
\hline 28 & UE-29 UZN \#9I & .01 & $11 / 15$ & & 63 & N70 4" Gage & NI & $11 / 15$ & & 99 & Weather station 4 (new) & .01 & $11 / 15$ & \\
\hline 28 & N91 4" Gage & NI & & & 64 & USW UZ-N71 & 0 & & & 99 & Weather station $4\left(8^{\prime \prime}\right)$ & NI & & \\
\hline 29 & USW UZ-N11 & NI & & & 64 & N71 4" Gage & NI & $11 / 15$ & & 100 & G-3 & .02 & $11 / 15$ & \\
\hline 29 & N11 4" Gage & NI & & & 65 & USW UZ-N72 & 0 & & & 100 & G-3 8" Gage & NI & & \\
\hline 30 & USW UZ-N15 & NI & & & 66 & USW UZ-N73 & .01 & $11 / 15$ & & 101 & Weather station 1 & $\mathbf{M}^{*}$ & & \\
\hline 31 & USW UZ-N16 & NI & & & 67 & USW UZ-N74 & .01 & $11 / 15$ & & 101 & Weather station $1\left(8^{\prime \prime}\right)$ & $\mathrm{NI}$ & & \\
\hline 32 & USW UZ-N17 & NI & & & 68 & USW UZ-N75 & 0 & $11 / 15$ & & 102 & Weather station 2 & NI & & \\
\hline 33 & USW UZ-N24 & 0 & $11 / 15$ & & 68 & N75 4" Gage & NI & $11 / 15$ & & 103 & Weather station 3 & .01 & $11 / 15$ & \\
\hline 33 & N24 4" Gage & NI & & & 69 & USW UZ-N76 & 0 & & & 103 & Weather station $3\left(8^{\prime \prime}\right)$ & NI & & \\
\hline 34 & USW UZ-N25 & 0 & $11 / 15$ & & 70 & USW UZ-N77 & $\mathbf{M}^{*}$ & $11 / 15$ & & 104 & Fran Ridge & $\mathbf{M}^{*}$ & & \\
\hline 35 & USW UZ-N26 & 0 & $11 / 15$ & & 70 & N77 4" Gage & NI & & & 104 & Fran Ridge (8") & NI & & \\
\hline 36 & USW UZ-N27 & 0 & $11 / 15$ & & 71 & USW UZ-N78 & $\mathbf{M}^{*}$ & & & 105 & Plug Hill & $\mathbf{M}^{*}$ & & \\
\hline 36 & N27 8" Gage & NI & & & 72 & USW UZ-N79 & $\mathbf{M}^{*}$ & & & 106 & 4JA ( $(8 ")$ & NI & & \\
\hline 37 & USW UZ-N35 & NI & & & 73 & USW UZ-N80 & $\mathbf{M}^{*}$ & & & 107 & Knothead Gap & NI & & \\
\hline 38 & USW UZ-N36 & NI & & & 73 & N80 4" Gage & $\mathrm{NI}$ & & & 108 & Upper Forty Mile & NI & & \\
\hline
\end{tabular}


Table 2. Summary of precipitation data from the nonrecording gage network at Yucca Mountain, Nevada, for water years 1992 and $1993--$ Continued

\begin{tabular}{|c|c|c|c|c|c|c|c|c|c|c|c|c|c|c|}
\hline $\begin{array}{l}\text { Loca- } \\
\text { tion } \\
\text { code }\end{array}$ & $\begin{array}{c}\text { Site } \\
\text { nomenclature }\end{array}$ & $\begin{array}{l}\text { Amount } \\
\text { (Inches) }\end{array}$ & $\begin{array}{l}\text { Date } \\
\text { read }\end{array}$ & Notes & $\begin{array}{l}\text { Loca- } \\
\text { tlon } \\
\text { code }\end{array}$ & $\begin{array}{c}\text { Site } \\
\text { nomenclature }\end{array}$ & $\begin{array}{l}\text { Amount } \\
\text { (inches) }\end{array}$ & $\begin{array}{l}\text { Date } \\
\text { read }\end{array}$ & Notes & $\begin{array}{l}\text { Loca- } \\
\text { tion } \\
\text { code }\end{array}$ & $\begin{array}{c}\text { Site } \\
\text { nomenclature }\end{array}$ & $\begin{array}{l}\text { Amount } \\
\text { (inches) }\end{array}$ & $\begin{array}{l}\text { Date } \\
\text { read }\end{array}$ & Notes \\
\hline \multicolumn{15}{|c|}{ STORM OF DECEMBER 7-8, 1991} \\
\hline 1 & UE-25 UZN \#1 & .11 & $12 / 9$ & & 39 & USW UZ-N37 & $\mathrm{M}^{*}$ & & & 74 & USW UZ-N81 & .12 & $12 / 10$ & *** \\
\hline 2 & UE-25 UZN \#2 & .13 & $12 / 9$ & & 39 & N37 4" Gage & NI & & & 75 & USW UZ-N82 & .12 & $12 / 10$ & $* *$ \\
\hline 3 & UE-25 UZN \#3 & .12 & $12 / 9$ & & 40 & USW UZ-N40 & .13 & $12 / 9$ & & 75 & N82 4" Gage & NI & & \\
\hline 4 & UE-25 UZN \#4 & .14 & $12 / 9$ & & 40 & N40 4" Gage & NI & & & 76 & USW UZ-N83 & .14 & $12 / 10$ & *** \\
\hline 4 & N4 4" Gage & NI & & & 41 & USW UZ-N41 & .13 & $12 / 9$ & & 77 & USW UZ-N84 & .14 & $12 / 10$ & ** \\
\hline 5 & UE-25 UZN \#5 & .12 & $12 / 9$ & & 42 & USW UZ-N42 & .12 & $12 / 9$ & & 78 & USW UZ-N86 & .16 & $12 / 10$ & ** \\
\hline 6 & UE-25 UZN \#6 & .12 & $12 / 9$ & & 43 & USW UZ-N43 & .11 & $12 / 9$ & & 79 & USW UZ-N87 & .15 & $12 / 10$ & $* *$ \\
\hline 7 & UE-25 UZN $\$ 7$ & .12 & $12 / 9$ & & 43 & N43 4" Gage & $\mathrm{NI}$ & & & 80 & USW UZ-N88 & .14 & $12 / 10$ & $* *$ \\
\hline 8 & UE-25 UZN \#8 & .12 & $12 / 9$ & & 44 & USW UZ-N44 & .13 & $12 / 9$ & & 81 & USW UZ-N89 & .17 & $12 / 10$ & $* *$ \\
\hline 9 & UE-25 UZN \#9 & .11 & $12 / 9$ & & 45 & USW UZ-N45 & .13 & $1,2 / 9$ & & 82 & USW UZ-N90 & .15 & $12 / 10$ & $* *$ \\
\hline 10 & UE-25 UZN $\# 10$ & .12 & $12 / 9$ & & 46 & USW UZ-N46 & .16 & $12 / 9$ & $* *$ & 82 & N90 4" Gage & NI & & \\
\hline 10 & N10 4" Gage & NI & & & 47 & USW UZ-N47 & .15 & $12 / 9$ & $* *$ & 83 & USW UZ-N93 & .16 & $12 / 9$ & \\
\hline 11 & UE-25 UZN \#12 & .14 & $12 / 9$ & & 48 & USW UZ-N48 & .16 & $12 / 9$ & ** & 84 & USW UZ-N94 & .15 & $12 / 9$ & \\
\hline 12 & UE-25 UZN \#13 & .11 & $12 / 9$ & & 49 & USW UZ-N49 & .13 & $12 / 9$ & ** & 85 & USW UZ-N95 & .16 & $12 / 9$ & \\
\hline 13 & UE-25 UZN \#14 & .12 & $12 / 9$ & & 50 & USW UZ-N50 & .14 & $12 / 9$ & $* *$ & 86 & USW UZ-N96 & .17 & $12 / 9$ & \\
\hline 14 & UE-25 UZN \#18 & .13 & $12 / 9$ & & 50 & N50 4' Gage & NI & & & 87 & USW UZ-N98 & .13 & $12 / 9$ & \\
\hline 15 & UE-25 UZN \#19 & .12 & $12 / 9$ & $* *$ & 51 & USW UZ-N51 & .13 & $12 / 9$ & & 87 & N98 4" Gage & $\mathrm{NI}$ & & \\
\hline 16 & UE-25 UZN \#20 & .14 & $12 / 9$ & $* *$ & 52 & USW UZ-N52 & .15 & $12 / 9$ & & 88 & USW UZ-13 & .14 & $12 / 9$ & \\
\hline 17 & UE-25 UZN \#21 & .14 & $12 / 9$ & $* *$ & 53 & USW UZ-N53 & .14 & $12 / 9$ & & 88 & UZ-13 4" Gage & NI & & \\
\hline 17 & N21 4" Gage & NI & & & 54 & USW UZ-N54 & $\mathbf{M}$ & & Broken & 89 & USW GA-1 & .21 & $12 / 9$ & \\
\hline 18 & UE-25 UZN $\# 22$ & .14 & $12 / 9$ & $* *$ & 54 & N54 4" Gage & NI & & & 90 & USW G-2 & .16 & $12 / 9$ & \\
\hline 19 & UE-25 UZN \#23 & .14 & $12 / 9$ & $* *$ & 55 & USW UZ-N55 & .13 & $12 / 9$ & & 91 & UE-25 WT-4 & .13 & $12 / 9$ & \\
\hline 20 & UE-25 UZN \#28 & .14 & $12 / 9$ & ** & 56 & USW UZ-N57 & .11 & $12 / 9$ & & 92 & UE-25 WT-18 & .12 & $12 / 9$ & \\
\hline 21 & UE-25 UZN \#29 & .15 & $12 / 9$ & $* *$ & 57 & USW UZ-N64 & NI & & & 93 & USW H-5 & .15 & $12 / 9$ & \\
\hline 22 & UE-25 UZN \#30 & .14 & $12 / 9$ & & 58 & USW UZ-N65 & .12 & $12 / 9$ & & 94 & HRF Wedge & $\mathbf{M}$ & & \\
\hline 23 & UE-25 UZN \#56 & .14 & $12 / 9$ & & 59 & USW UZ-N66 & .12 & $12 / 9$ & & 94 & HRF 4" Gage & .10 & $12 / 9$ & \\
\hline 24 & UE-25 UZN $\# 60$ & .12 & $12 / 9$ & & 60 & USW UZ-N67 & .12 & $12 / 9$ & & 94 & HRF 8" Gage & .11 & $12 / 9$ & \\
\hline 25 & UE-25 UZN \#85 & .13 & $12 / 9$ & & 60 & N67 4" Gage & NI & & & 95 & USW H-3 & .11 & $12 / 9$ & \\
\hline 26 & UE-25 UZN \#92 & .10 & $12 / 10$ & & 61 & USW UZ-N68 & .11 & $12 / 9$ & & 96 & Weather station 4 (old) & .11 & $12 / 9$ & \\
\hline 26 & N92 4" Gage & NI & & & 62 & USW UZ-N69 & .11 & $12 / 9$ & & 97 & Weather station 5 (Prow) & $\mathrm{M}$ & & \\
\hline
\end{tabular}


Table 2. Summary of precipitation data from the nonrecording gage network at Yucca Mountain, Nevada, for water years 1992 and 1993 --Continued

\begin{tabular}{|c|c|c|c|c|c|c|c|c|c|c|c|c|c|c|}
\hline $\begin{array}{l}\text { Loca- } \\
\text { tion } \\
\text { code }\end{array}$ & $\begin{array}{c}\text { Site } \\
\text { nomenclature }\end{array}$ & $\begin{array}{l}\text { Amount } \\
\text { (inches) }\end{array}$ & $\begin{array}{l}\text { Date } \\
\text { read }\end{array}$ & Notes & $\begin{array}{l}\text { Loca- } \\
\text { tion } \\
\text { code }\end{array}$ & $\begin{array}{c}\text { Site } \\
\text { nomenclature }\end{array}$ & $\begin{array}{l}\text { Amount } \\
\text { (inches) }\end{array}$ & $\begin{array}{l}\text { Date } \\
\text { read }\end{array}$ & Notes & $\begin{array}{l}\text { Loca- } \\
\text { tion } \\
\text { code }\end{array}$ & $\begin{array}{c}\text { Site } \\
\text { nomenclature }\end{array}$ & $\begin{array}{l}\text { Amount } \\
\text { (inches) }\end{array}$ & $\begin{array}{l}\text { Date } \\
\text { read }\end{array}$ & Notes \\
\hline \multicolumn{15}{|c|}{ STORM OF DECEMBER 7-8, 1991-Continued } \\
\hline 27 & UE-25 UZN \#97 & .13 & $12 / 9$ & ** & 63 & USW UZ-N70 & .15 & $12 / 9$ & & 98 & Evaporation pan & .08 & $12 / 9$ & \\
\hline 28 & UE-29 UZN \#91 & $\mathbf{v}$ & $12 / 10$ & & 63 & N70 4" Gage & NI & & & 99 & Weather station 4 (new) & .14 & $12 / 9$ & \\
\hline 28 & N91 4" Gage & NI & & & 64 & USW UZ-N71 & .14 & $12 / 9$ & & 99 & Wx Sta $4\left(8^{\prime \prime}\right)$ & NI & & \\
\hline 29 & USW UZ-N11 & NI & & & 64 & N71 4" Gage & NI & & & 100 & G-3 & .13 & $12 / 9$ & \\
\hline 29 & N11 4" Gage & NI & & & 65 & USW UZ-N72 & .14 & $12 / 9$ & & 100 & G-3 (8") & $\mathrm{NI}$ & & \\
\hline 30 & USW UZ-N15 & NI & & & 66 & USW UZ-N73 & .14 & $12 / 9$ & & 101 & Weather station 1 & .10 & $12 / 9$ & $* *$ \\
\hline 31 & USW UZ-N16 & NI & & & 67 & USW UZ-N74 & .14 & $12 / 9$ & & 101 & Weather station $1(8 ")$ & NI & & \\
\hline 32 & USW UZ-N17 & NI & & & 68 & USW UZ-N75 & .15 & $12 / 9$ & & 102 & Weather station 2 & NI & & \\
\hline 33 & USW UZ-N24 & .13 & $12 / 9$ & & 68 & N75 4" Gage & NI & & & 103 & Weather station 3 & .15 & $12 / 9$ & \\
\hline 33 & N24 4" Gage & NI & & & 69 & USW UZ-N76 & .16 & $12 / 9$ & & 103 & Weather station $3\left(8^{\prime \prime}\right)$ & NI & & \\
\hline 34 & USW UZ-N25 & .13 & $12 / 10$ & & 70 & USW UZ-N77 & .09 & $12 / 10$ & $* *$ & 104 & Fran Ridge & .35 & & $* *$ \\
\hline 35 & USW UZ-N26 & .13 & $12 / 9$ & & 70 & N77 4" Gage & NI & & & 104 & Fran Ridge (8") & NI & & \\
\hline 36 & USW UZ-N27 & 0.17 & $12 / 9$ & & 71 & USW UZ-N78 & .17 & $12 / 10$ & $* *$ & 105 & Plug Hill & .12 & $12 / 10$ & $* *$ \\
\hline 36 & N27 8" Gage & $\mathrm{NI}$ & & & 72 & USW UZ-N79 & .17 & $12 / 10$ & $* *$ & 106 & 4JA (8") & NI & & \\
\hline 37 & USW UZ-N35 & NI & & & 73 & USW UZ-N80 & .15 & $12 / 10$ & $* *$ & 107 & Knothead Gap & NI & & \\
\hline 38 & USW UZ-N36 & $\mathrm{NI}$ & & & 73 & N80 4" Gage & $\mathrm{NI}$ & & & 108 & Upper Fortymile & NI & & \\
\hline
\end{tabular}


Table 2. Summary of precipitation data from the nonrecording gage network at Yucca Mountain, Nevada, for water years 1992 and $1993--$ Continued

\begin{tabular}{|c|c|c|c|c|c|c|c|c|c|c|c|c|c|c|}
\hline $\begin{array}{l}\text { Loca- } \\
\text { tion } \\
\text { code }\end{array}$ & $\begin{array}{c}\text { Site } \\
\text { nomenclature }\end{array}$ & $\begin{array}{l}\text { Amount } \\
\text { (inches) }\end{array}$ & $\begin{array}{l}\text { Date } \\
\text { read }\end{array}$ & Notes & $\begin{array}{l}\text { Loca- } \\
\text { tion } \\
\text { code }\end{array}$ & $\begin{array}{c}\text { Site } \\
\text { nomenclature }\end{array}$ & $\begin{array}{l}\text { Amount } \\
\text { (inches) }\end{array}$ & $\begin{array}{l}\text { Dale } \\
\text { read }\end{array}$ & Notes & $\begin{array}{l}\text { Loca- } \\
\text { tion } \\
\text { code }\end{array}$ & $\begin{array}{c}\text { Site } \\
\text { nomenclature }\end{array}$ & $\begin{array}{l}\text { Amount } \\
\text { (Inches) }\end{array}$ & $\begin{array}{l}\text { Date } \\
\text { read }\end{array}$ & Notes \\
\hline \multicolumn{15}{|c|}{ STORM OF DECEMBER 19, 1991} \\
\hline 1 & UE-25 UZN \#1 & .13 & $12 / 19$ & & 39 & USW UZ-N37 & .21 & $12 / 19$ & & 74 & USW UZ-N81 & $\mathrm{M}^{*}$ & & \\
\hline 2 & UE-25 UZN \#2 & .17 & $12 / 19$ & & 39 & N37 4" Gage & NI & & & 75 & USW UZ-N82 & $\mathrm{M}^{*}$ & & \\
\hline 3 & UE-25 UZN \#3 & .19 & $12 / 19$ & & 40 & USW UZ-N40 & .09 & $12 / 19$ & & 75 & N82 4" Gage & NI & & \\
\hline 4 & UE-25 UZN \#4 & .16 & $12 / 19$ & & 40 & N40 4" Gage & NI & & & 76 & USW UZ-N83 & $\mathbf{M}^{*}$ & & \\
\hline 4 & N4 4" Gage & NI & & & 41 & USW UZ-N41 & .08 & $12 / 19$ & & 77 & USW UZ-N84 & $\mathbf{M}^{*}$ & & \\
\hline 5 & UE-25 UZN \#5 & .18 & $12 / 19$ & & 42 & USW UZ-N42 & .10 & $12 / 19$ & & 78 & USW UZ-N86 & $\mathbf{M}^{*}$ & & \\
\hline 6 & UE-25 UZN \#6 & .18 & $12 / 19$ & & 43 & USW UZ-N43 & .08 & $12 / 19$ & & 79 & USW UZ-N87 & $\mathbf{M}^{*}$ & & \\
\hline 7 & UE-25 UZN \#7 & .19 & $12 / 19$ & & 43 & N43 4" Gage & NI & & & 80 & USW UZ-N88 & $\mathrm{M}^{*}$ & & \\
\hline 8 & UE-25 UZN \#8 & .14 & $12 / 19$ & & 44 & USW UZ-N44 & .07 & $12 / 19$ & & 81 & USW UZ-N89 & $\mathrm{M}^{*}$ & & \\
\hline 9 & UE-25 UZN \#9 & .19 & $12 / 19$ & & 45 & USW UZ-N45 & .06 & $12 / 19$ & & 82 & USW UZ-N90 & $\mathbf{M}^{*}$ & & \\
\hline 10 & UE-25 UZN \#10 & .12 & $12 / 19$ & & 46 & USW UZ-N46 & .04 & $12 / 19$ & & 82 & N90 4" Gage & NI & & \\
\hline 10 & N10 4" Gage & NI & & & 47 & USW UZ-N47 & .07 & $12 / 19$ & & 83 & USW UZ-N93 & 0 & $12 / 19$ & \\
\hline 11 & UE-25 UZN \#12 & .19 & $12 / 19$ & & 48 & USW UZ-N48 & .10 & $12 / 19$ & & 84 & USW UZ-N94 & .02 & $12 / 19$ & \\
\hline 12 & UE-25 UZN \#13 & .19 & $12 / 19$ & & 49 & USW UZ-N49 & .12 & $12 / 19$ & & 85 & USW UZ-N95 & .07 & $12 / 19$ & \\
\hline 13 & UE-25 UZN \#14 & .16 & $12 / 19$ & & 50 & USW UZ-N50 & .08 & $12 / 19$ & & 86 & USW UZ-N96 & .01 & $12 / 19$ & \\
\hline 14 & UE-25 UZN \#18 & .13 & $12 / 19$ & & 50 & N50 4' Gage & NI & & & 87 & USW UZ-N98 & .06 & $12 / 19$ & \\
\hline 15 & UE-25 UZN \#19 & .11 & $12 / 19$ & & 51 & USW UZ-N51 & .08 & $12 / 19$ & & 87 & N98 4" Gage & NI & & \\
\hline 16 & UE-25 UZN \#20 & .12 & $12 / 19$ & & 52 & USW UZ-N52 & .06 & $12 / 19$ & & 88 & USW UZ-13 & .04 & $12 / 19$ & \\
\hline 17 & UE-25 UZN \#21 & .10 & $12 / 19$ & & 53 & USW UZ-N53 & .10 & $12 / 19$ & & 88 & UZ-13 4" Gage & NI & $12 / 19$ & \\
\hline 17 & N21 4" Gage & $\mathrm{NI}$ & & & 54 & USW UZ-N54 & $\mathbf{M}$ & & & 89 & USW GA-1 & .01 & $12 / 19$ & \\
\hline 18 & UE-25 UZN \#22 & .10 & $12 / 19$ & & 54 & N54 4" Gage & .15 & $12 / 19$ & & 90 & USW G-2 & .02 & $12 / 19$ & \\
\hline 19 & UE-25 UZN $\# 23$ & .10 & $12 / 19$ & & 55 & USW UZ-N5S & .05 & $12 / 19$ & & 91 & UE-25 WT-4 & .16 & $12 / 19$ & \\
\hline 20 & UE-25 UZN $\# 28$ & .13 & $12 / 19$ & & 56 & USW UZ-N57 & .12 & & & 92 & UE-25 WT-18 & .03 & $12 / 19$ & \\
\hline 21 & UE-25 UZN \#29 & .12 & $12 / 19$ & & 57 & USW UZ-N64 & NI & & & 93 & USW H-5 & 0 & $12 / 19$ & \\
\hline 22 & UE-25 UZN $\# 30$ & .13 & $12 / 19$ & & 58 & USW UZ-N65 & .05 & & & 94 & HRF Wedge & & & Removed \\
\hline 23 & UE-25 UZN \#56 & .15 & $12 / 19$ & & 59 & USW UZ-N66 & .07 & $12 / 19$ & & 94 & HRF 4" Gage & .13 & $12 / 19$ & \\
\hline 24 & UE-25 UZN $\# 60$ & .15 & $12 / 19$ & & 60 & USW UZ-N67 & .10 & $12 / 19$ & & 94 & HRF 8" Gage & .12 & $12 / 19$ & \\
\hline 25 & UE-25 UZN \#85 & .08 & $12 / 19$ & & 60 & N67 4" Gage & NI & & & 95 & USW H-3 & .04 & $12 / 19$ & \\
\hline 26 & UE-25 UZN \#92 & $\mathrm{M}^{*}$ & & & 61 & USW UZ-N68 & .12 & $12 / 19$ & & 96 & Weather station 4 (old) & .04 & $12 / 19$ & \\
\hline 26 & N92 4" Gage & NI & & & 62 & USW UZ-N69 & .10 & $12 / 19$ & & 97 & Weather station 5 (Prow) & $\mathbf{M}$ & & \\
\hline
\end{tabular}


Table 2. Summary of precipitation data from the nonrecording gage network at Yucca Mountain, Nevada, for water years 1992 and $1993--$ Continued

\begin{tabular}{|c|c|c|c|c|c|c|c|c|c|c|c|c|c|c|}
\hline $\begin{array}{l}\text { Loca- } \\
\text { tion } \\
\text { code }\end{array}$ & $\begin{array}{c}\text { Site } \\
\text { nomenclature }\end{array}$ & $\begin{array}{l}\text { Amount } \\
\text { (inches) }\end{array}$ & $\begin{array}{l}\text { Date } \\
\text { read }\end{array}$ & Notes & $\begin{array}{l}\text { Loca- } \\
\text { tion } \\
\text { code }\end{array}$ & $\begin{array}{c}\text { Site } \\
\text { nomenclature }\end{array}$ & $\begin{array}{l}\text { Amount } \\
\text { (inches) }\end{array}$ & $\begin{array}{l}\text { Date } \\
\text { read }\end{array}$ & Notes & $\begin{array}{l}\text { Loca- } \\
\text { tion } \\
\text { code }\end{array}$ & $\begin{array}{c}\text { Site } \\
\text { nomenclature }\end{array}$ & $\begin{array}{l}\text { Amount } \\
\text { (Inches) }\end{array}$ & $\begin{array}{l}\text { Date } \\
\text { read }\end{array}$ & Notes \\
\hline \multicolumn{15}{|c|}{ STORM OF DECEMBER 19, 1991-Continued } \\
\hline 27 & UE-25 UZN \#97 & .16 & $12 / 19$ & & 63 & USW UZ-N70 & .05 & $12 / 19$ & & 98 & Evaporation pan & .10 & $12 / 19$ & \\
\hline 28 & UE-29 UZN \#91 & .08 & $12 / 2 ?$ & & 63 & N70 4" Gage & NI & & & 99 & Weather station 4 (new) & .05 & $12 / 19$ & \\
\hline 28 & N91 4" Gage & NI & & & 64 & USW UZ-N71 & .06 & $12 / 19$ & & 99 & Wx Sta $4(8 ")$ & NI & & \\
\hline 29 & USW UZ-N11 & NI & & & 64 & N71 4" Gage & NI & & & 100 & G-3 & .02 & $12 / 19$ & \\
\hline 29 & N11 4" Gage & NI & & & 65 & USW UZ-N72 & $\mathbf{M}^{*}$ & & & 100 & G-3 (8") & NI & & \\
\hline 30 & USW UZ-N15 & NI & & & 66 & USW UZ-N73 & $\mathbf{M}^{*}$ & & & 101 & Weather station 1 & .14 & $12 / 19$ & \\
\hline 31 & USW UZ-N16 & NI & & & 67 & USW UZ-N74 & .04 & $12 / 19$ & & 101 & Weather station $1\left(8^{\prime \prime}\right)$ & NI & & \\
\hline 32 & USW UZ-N17 & $\mathrm{NI}$ & & & 68 & USW UZ-N75 & $\mathbf{M}^{*}$ & & & 102 & Weather station 2 & $\mathrm{NI}$ & & \\
\hline 33 & USW UZ-N24 & .07 & $12 / 19$ & & 68 & N75 4" Gage & NI & & & 103 & Weather station 3 & .05 & $12 / 19$ & \\
\hline 33 & N24 4" Gage & $\mathrm{NI}$ & & & 69 & USW UZ-N76 & $\mathbf{M}^{*}$ & & & 103 & Weather station $3\left(8^{\prime \prime}\right)$ & $\mathrm{NI}$ & & \\
\hline 34 & USW UZ-N25 & .06 & $12 / 19$ & & 70 & USW UZ-N77 & $\mathbf{M}^{*}$ & & & 104 & Fran Ridge & $\mathbf{M}^{*}$ & & \\
\hline 35 & USW UZ-N26 & .05 & $12 / 19$ & & 70 & N77 4" Gage & NI & & & 104 & Fran Ridge & NI & & \\
\hline 36 & USW UZ-N27 & 0 & & & 71 & USW UZ-N78 & $\mathbf{M}^{*}$ & & & 105 & Plug Hill & $\mathbf{M}^{*}$ & & \\
\hline 36 & N27 8" Gage & $\mathrm{NI}$ & & & 72 & USW UZ-N79 & $\mathrm{M}^{*}$ & & & 106 & 4JA (8") & NI & & \\
\hline 37 & USW UZ-N35 & NI & & & 73 & USW UZ-N80 & $\mathbf{M}^{*}$ & & & 107 & Knothead Gap & $\mathrm{NI}$ & & \\
\hline 38 & USW UZ-N36 & NI & & & 73 & N80 4" Gage & $\mathrm{NI}$ & & & 108 & Upper Fortymile & NI & & \\
\hline
\end{tabular}


Table 2. Summary of precipitation data from the nonrecording gage network at Yucca Mountain, Nevada, for water years 1992 and $1993--$ Continued

\begin{tabular}{|c|c|c|c|c|c|c|c|c|c|c|c|c|c|c|}
\hline $\begin{array}{l}\text { Loca- } \\
\text { fion } \\
\text { code }\end{array}$ & $\begin{array}{c}\text { Site } \\
\text { nomenclature }\end{array}$ & $\begin{array}{l}\text { Amount } \\
\text { (inches) }\end{array}$ & $\begin{array}{l}\text { Date } \\
\text { read }\end{array}$ & Notes & $\begin{array}{l}\text { Loca- } \\
\text { tion } \\
\text { code }\end{array}$ & $\begin{array}{c}\text { Site } \\
\text { nomenclature }\end{array}$ & $\begin{array}{l}\text { Amount } \\
\text { (inches) }\end{array}$ & $\begin{array}{l}\text { Date } \\
\text { read }\end{array}$ & Notes & $\begin{array}{l}\text { Loca- } \\
\text { tion } \\
\text { code }\end{array}$ & $\begin{array}{c}\text { Site } \\
\text { nomenclature }\end{array}$ & $\begin{array}{l}\text { Amount } \\
\text { (inches) }\end{array}$ & $\begin{array}{l}\text { Date } \\
\text { read }\end{array}$ & Notes \\
\hline \multicolumn{15}{|c|}{ STORM OF DECEMBER 28-30, 1991} \\
\hline 1 & UE-25 UZN \#1 & .46 & $12 / 30$ & & 39 & USW UZ-N37 & .47 & $12 / 30$ & & 74 & USW UZ-N81 & $\mathrm{M}^{*}$ & & \\
\hline 2 & UE-25 UZN \#2 & .49 & $12 / 30$ & & 39 & N37 4" Gage & NI & & & 75 & USW UZ-N82 & $\mathrm{M}^{*}$ & & \\
\hline 3 & UE-25 UZN \#3 & .47 & $12 / 30$ & & 40 & USW UZ-N40 & .49 & $12 / 30$ & & 75 & N82 4" Gage & NI & & \\
\hline 4 & UE-25 UZN \#4 & .47 & $12 / 30$ & & 40 & N40 4" Gage & NI & & & 76 & USW UZ-N83 & $\mathbf{M}^{*}$ & & \\
\hline 4 & N4 4" Gage & NI & & & 41 & USW UZ-N41 & .54 & $12 / 30$ & & 77 & USW UZ-N84 & $\mathbf{M}^{*}$ & & \\
\hline 5 & UE-25 UZN \#5 & .49 & $12 / 30$ & & 42 & USW UZ-N42 & .48 & $12 / 30$ & & 78 & USW UZ-N86 & $\mathrm{M}^{*}$ & & \\
\hline 6 & UE-25 UZN \#6 & .47 & $12 / 30$ & & 43 & USW UZ-N43 & .54 & $12 / 30$ & & 79 & USW UZ-N87 & $\mathbf{M}^{*}$ & & \\
\hline 7 & UE-25 UZN \#7 & .49 & $12 / 30$ & & 43 & N43 4" Gage & NI & & & 80 & USW UZ-N88 & & & \\
\hline 8 & UE-25 UZN \#8 & .48 & $12 / 30$ & & 44 & USW UZ-N44 & .50 & $12 / 30$ & & 81 & USW UZ-N89 & $\mathrm{M}^{*}$ & & \\
\hline 9 & UE-25 UZN \#9 & .47 & $12 / 30$ & & 45 & USW UZ-N45 & .50 & $12 / 30$ & & 82 & USW UZ-N90 & $\mathrm{M}^{*}$ & & \\
\hline 10 & UE- 25 UZN $\# 10$ & .51 & $12 / 30$ & & 46 & USW UZ-N46 & .56 & $12 / 30$ & & 82 & N90 4" Gage & NI & & \\
\hline 10 & N10 4" Gage & NI & & & 47 & USW UZ-N47 & .63 & $12 / 30$ & & 83 & USW UZ-N93 & .36 & $12 / 30$ & \\
\hline 11 & UE-25 UZN \#12 & .47 & $12 / 30$ & & 48 & USW UZ-N48 & .58 & $12 / 30$ & & 84 & USW UZ-N94 & .38 & $12 / 30$ & \\
\hline 12 & UE-25 UZN \#13. & $\mathrm{M}$ & & & 49 & USW UZ-N49 & .50 & $12 / 30$ & & 85 & USW UZ-N95 & .40 & $12 / 30$ & \\
\hline 13 & UE-25 UZN \#14 & .48 & $12 / 30$ & & 50 & USW UZ-N50 & .55 & $12 / 30$ & & 86 & USW UZ-N96 & .33 & $12 / 30$ & \\
\hline 14 & UE-25 UZN \#18 & .48 & $12 / 30$ & & 50 & N50 4' Gage & NI & & & 87 & USW UZ-N98 & .55 & $12 / 30$ & \\
\hline 15 & UE-25 UZN $\# 19$ & .55 & $12 / 30$ & & 51 & USW UZ-N51 & .50 & $12 / 30$ & & 87 & N98 4" Gage & $\mathrm{NI}$ & & \\
\hline 16 & UE-25 UZN \#20 & .52 & $12 / 30$ & & 52 & USW UZ-N52 & .54 & $12 / 30$ & & 88 & USW UZ-13 & .40 & $12 / 30$ & \\
\hline 17 & UE-25 UZN $\# 21$ & .52 & $12 / 30$ & & 53 & USW UZ-N53 & .58 & $12 / 30$ & & 88 & UZ-13 4" Gage & NI & & \\
\hline 17 & N21 4" Gage & NI & & & 54 & USW UZ-N54 & $\mathbf{M}$ & & & 89 & USW GA-1 & $\mathrm{M}^{*}$ & & \\
\hline 18 & UE-25 UZN $\# 22$ & .54 & $12 / 30$ & & 54 & N54 4" Gage & NI & & & 90 & USW G-2 & $\mathrm{M}^{*}$ & & \\
\hline 19 & UE-25 UZN \#23 & .52 & $12 / 30$ & & 55 & USW UZ-N55 & .52 & $12 / 30$ & & 91 & UE-25 WT-4 & .47 & $12 / 30$ & \\
\hline 20 & UE-25 UZN \#28 & $\mathrm{M}^{*}$ & & & 56 & USW UZ-N57 & $\mathbf{M}^{*}$ & & & 92 & UE-25 WT-18 & .48 & $12 / 30$ & \\
\hline 21 & UE-25 UZN \#29 & .54 & $12 / 30$ & & 57 & USW UZ-N64 & NI & & & 93 & USW H-5 & .37 & $12 / 30$ & \\
\hline 22 & UE-25 UZN \#30 & .50 & $12 / 30$ & & 58 & USW UZ-N65 & .52 & $12 / 30$ & & 94 & HRF Wedge & & & Removed \\
\hline 23 & UE-25 UZN \#56 & .54 & $12 / 30$ & & 59 & USW UZ-N66 & .50 & $12 / 30$ & & 94 & HRF 4" Gage & .41 & $12 / 30$ & \\
\hline 24 & UE-25 UZN \#60 & .50 & $12 / 30$ & & 60 & USW UZ-N67 & .55 & $12 / 30$ & & 94 & HRF 8" Gage & .41 & $12 / 30$ & \\
\hline 25 & UE-25 UZN \#85 & .54 & $12 / 30$ & & 60 & N67 4" Gage & NI & & & 95 & USW H-3 & .29 & $12 / 30$ & \\
\hline 26 & UE-25 UZN \#92 & $\mathrm{M}^{*}$ & & & 61 & USW UZ-N68 & .53 & $12 / 30$ & & 96 & Weather station 4 (old) & .32 & $12 / 30$ & \\
\hline 26 & N92 4" Gage & NI & & & 62 & USW UZ-N69 & .49 & $12 / 30$ & & 97 & Weather station 5 (Prow) & M & & \\
\hline
\end{tabular}


N Table 2. Summary of precipitation data from the nonrecording gage network at Yucca Mountain, Nevada, for water years 1992 and $1993--$ Continued

\begin{tabular}{|c|c|c|c|c|c|c|c|c|c|c|c|c|c|c|}
\hline $\begin{array}{l}\text { Loca- } \\
\text { tion } \\
\text { code }\end{array}$ & $\begin{array}{c}\text { Site } \\
\text { nomenclature }\end{array}$ & $\begin{array}{l}\text { Amount } \\
\text { (inches) }\end{array}$ & $\begin{array}{l}\text { Date } \\
\text { read }\end{array}$ & Notes & $\begin{array}{l}\text { Loca- } \\
\text { lion } \\
\text { code }\end{array}$ & $\begin{array}{c}\text { Site } \\
\text { nomenclature }\end{array}$ & $\begin{array}{l}\text { Amount } \\
\text { (inches) }\end{array}$ & $\begin{array}{l}\text { Date } \\
\text { read }\end{array}$ & Notes & $\begin{array}{l}\text { Loca- } \\
\text { tion } \\
\text { code }\end{array}$ & $\begin{array}{c}\text { Site } \\
\text { nomenclature }\end{array}$ & $\begin{array}{l}\text { Amount } \\
\text { (inches) }\end{array}$ & $\begin{array}{l}\text { Date } \\
\text { read }\end{array}$ & Notes \\
\hline \multicolumn{15}{|c|}{ STORM OF DECEMBER 28-30, 1991-Continued } \\
\hline 27 & UE-25 UZN $\# 97$ & $\mathbf{M}^{*}$ & & & 63 & USW UZ-N70 & .51 & $12 / 30$ & & 98 & Evaporation pan & .37 & $12 / 30$ & \\
\hline 28 & UE-29 UZN \#91 & $\mathbf{M}^{*}$ & & & 63 & N70 4" Gage & $\mathrm{NI}$ & & & 99 & Weather station 4 (new) & .36 & $12 / 30$ & \\
\hline 28 & N91 4" Gage & NI & & & 64 & USW UZ-N71 & .34 & $12 / 30$ & & 99 & Wx Sta $4(8 ")$ & NI & & \\
\hline 29 & USW UZ-N11 & NI & & & 64 & N71 4" Gage & NI & & & 100 & G-3 & .52 & $12 / 30$ & \\
\hline 29 & N11 4" Gage & NI & & & 65 & USW UZ-N72 & .41 & $12 / 30$ & $* *$ & 100 & G-3 (8") & NI & & \\
\hline 30 & USW UZ-N15 & NI & & & 66 & USW UZ-N73 & .54 & $12 / 30$ & $* *$ & 101 & Weather station 1 & .52 & $12 / 30$ & \\
\hline 31 & USW UZ-N16 & NI & & & 67 & USW UZ-N74 & .31 & $12 / 30$ & & 101 & Weather station $1(8 ")$ & .62 & $12 / 30$ & \\
\hline 32 & USW UZ-N17 & NI & & & 68 & USW UZ-N75 & .41 & $12 / 30$ & $* *$ & 102 & Weather station 2 & NI & & \\
\hline 33 & USW UZ-N24 & .57 & $12 / 30$ & & 68 & N75 4" Gage & NI & & & 103 & Weather station 3 & NI & & \\
\hline 33 & N24 4" Gage & $\mathrm{NI}$ & & & 69 & USW UZ-N76 & .38 & $12 / 30$ & $* *$ & 103 & Weather station 3 ( $8 ")$ & NI & & \\
\hline 34 & USW UZ-N25 & .58 & $12 / 30$ & & 70 & USW UZ-N77 & $M^{*}$ & & & 104 & Fran Ridge & $\mathbf{M}^{*}$ & & \\
\hline 35 & USW UZ-N26 & .57 & $12 / 30$ & & 70 & N77 4" Gage & NI & & & 104 & Fran Ridge (8") & NI & & \\
\hline 36 & USW UZ-N27 & .48 & $12 / 30$ & & 71 & USW UZ-N78 & $\mathbf{M}^{*}$ & & & 105 & Plug Hill & $\mathbf{M}^{*}$ & & \\
\hline 36 & N27 8" Gage & $\mathrm{NI}$ & & & 72 & USW UZ-N79 & $\mathbf{M}^{*}$ & & & 106 & 4JA (8") & NI & & \\
\hline 37 & USW UZ-N35 & NI & & & 73 & USW UZ-N80 & $\mathbf{M}^{*}$ & & & 107 & Knothead Gap & NI & & \\
\hline 38 & USW UZ-N36 & $\mathrm{NI}$ & & & 73 & N80 4" Gage & NI & & & 108 & Upper Fortymile & NI & & \\
\hline
\end{tabular}


Table 2. Summary of precipitation data from the nonrecording gage network at Yucca Mountain, Nevada, for water years 1992 and $1993--$ Continued

\begin{tabular}{|c|c|c|c|c|c|c|c|c|c|c|c|c|c|c|}
\hline $\begin{array}{l}\text { Loca- } \\
\text { tion } \\
\text { code }\end{array}$ & $\begin{array}{c}\text { Site } \\
\text { nomenefature }\end{array}$ & $\begin{array}{l}\text { Amount } \\
\text { (inches) }\end{array}$ & $\begin{array}{l}\text { Date } \\
\text { read }\end{array}$ & Notes & $\begin{array}{l}\text { Loca- } \\
\text { tion } \\
\text { code }\end{array}$ & $\begin{array}{c}\text { Slte } \\
\text { nomenclature }\end{array}$ & $\begin{array}{l}\text { Amount } \\
\text { (inches) }\end{array}$ & $\begin{array}{l}\text { Date } \\
\text { read }\end{array}$ & Notes & $\begin{array}{l}\text { Loca- } \\
\text { tion } \\
\text { code }\end{array}$ & $\begin{array}{c}\text { Site } \\
\text { nomenclature }\end{array}$ & $\begin{array}{l}\text { Amount } \\
\text { (inches) }\end{array}$ & $\begin{array}{l}\text { Date } \\
\text { read }\end{array}$ & Notes \\
\hline \multicolumn{15}{|c|}{ STORM OF JANUARY 3-5, 1992} \\
\hline 1 & UE-25 UZN \#1 & 1.19 & $1 / 7$ & & 39 & USW UZ-N37 & 1.24 & $1 / 7$ & & 74 & USW UZ-N81 & 1.88 & $1 / 24$ & $* *$ \\
\hline 2 & UE-25 UZN \#2 & 1.21 & $1 / 7$ & & 39 & N37 4" Gage & NI & & & 75 & USW UZ-N82 & 1.70 & $1 / 24$ & *** \\
\hline 3 & UE-25 UZN \#3 & 1.22 & $1 / 7$ & & 40 & USW UZ-N40 & 1.29 & $1 / 7$ & & 75 & N82 4" Gage & NI & & \\
\hline 4 & UE-25 UZN \#4 & 1.28 & $1 / 7$ & & 40 & N40 4" Gage & $\mathrm{NI}$ & & & 76 & USW UZ-N83 & 1.94 & $1 / 24$ & ** \\
\hline 4 & N4 4" Gage & $\mathrm{NI}$ & & & 41 & USW UZ-N41 & 1.30 & $1 / 7$ & & 77 & USW UZ-N84 & 1.85 & $1 / 24$ & ** \\
\hline 5 & UE-25 UZN \#5 & 1.21 & $1 / 7$ & & 42 & USW UZ-N42 & 1.27 & $1 / 7$ & & 78 & USW UZ-N86 & 2.00 & $1 / 24$ & ** \\
\hline 6 & UE-25 UZN \#6 & 1.22 & $1 / 7$ & & 43 & USW UZ-N43 & 1.27 & $1 / 7$ & & 79 & USW UZ-N87 & 1.90 & $1 / 24$ & $* *$ \\
\hline 7 & UE-25 UZN \#7 & 1.27 & $1 / 7$ & & 43 & N43 4" Gage & NI & & & 80 & USW UZ-N88 & 1.88 & $1 / 24$ & ** \\
\hline 8 & UE-25 UZN \#8 & 1.27 & $1 / 7$ & & 44 & USW UZ-N44 & 1.25 & $1 / 7$ & & 81 & USW UZ-N89 & 1.88 & $1 / 24$ & $* *$ \\
\hline 9 & UE-25 UZN \#9 & 1.24 & $1 / 7$ & & 45 & USW UZ-N45 & 1.27 & $1 / 7$ & & 82 & USW UZ-N90 & 1.80 & $1 / 24$ & $* *$ \\
\hline 10 & UE-25 UZN \#10 & 1.27 & $1 / 7$ & & 46 & USW UZ-N46 & 1.40 & $1 / 7$ & & 82 & N90 4" Gage & NI & & \\
\hline 10 & N10 4" Gage & NI & & & 47 & USW UZ-N47 & 1.40 & $1 / 7$ & & 83 & USW UZ-N93 & .95 & $1 / 8$ & \\
\hline 11 & UE-25 UZN \#12 & 1.24 & $1 / 7$ & & 48 & USW UZ-N48 & 1.29 & $1 / 7$ & & 84 & USW UZ-N94 & 1.06 & $1 / 8$ & \\
\hline 12 & UE-25 UZN \#13 & $\mathbf{M}$ & & & 49 & USW UZ-N49 & 1.28 & $1 / 7$ & & 85 & USW UZ-N95 & .98 & $1 / 8$ & \\
\hline 13 & UE-25 UZN \#14 & 1.11 & $1 / 15$ & FZN & 50 & USW UZ-N50 & 1.26 & $1 / 7$ & & 86 & USW UZ-N96 & .90 & $1 / 8$ & \\
\hline 14 & UE-25 UZN \#18 & 1.25 & $1 / 7$ & & 50 & N50 4" Gage & NI & & & 87 & USW UZ-N98 & 1.36 & $1 / 7$ & \\
\hline 15 & UE-25 UZN \#19 & 1.32 & $1 / 7$ & & 51 & USW UZ-N51 & 1.25 & $1 / 7$ & & 87 & N98 4" Gage & $\mathrm{NI}$ & & \\
\hline 16 & UE-25 UZN \#20 & 1.26 & $1 / 7$ & & 52 & USW UZ-N52 & 1.29 & $1 / 7$ & & 88 & USW UZ-13 & .95 & $1 / 8$ & \\
\hline 17 & UE-25 UZN \#21 & 1.26 & $1 / 7$ & & 53 & USW UZ-N53 & 1.27 & $1 / 7$ & & 88 & UZ-13 4" Gage & NI & & \\
\hline 17 & N21 4" Gage & NI & & & 54 & USW UZ-N54 & $\mathbf{M}$ & & & 89 & USW GA-1 & 1.53 & $1 / 15$ & $\mathrm{FZN}^{* *}$ \\
\hline 18 & UE-25 UZN \#22 & 1.25 & $1 / 7$ & & 54 & N54 4" Gage & NI & & & 90 & USW G-2 & 1.63 & $1 / 15$ & $\mathrm{FZN}^{* *}$ \\
\hline 19 & UE-25 UZN $\# 23$ & 1.23 & $1 / 7$ & & 55 & USW UZ-N55 & 1.14 & $1 / 7$ & & 91 & UE-25 WT-4 & 1.24 & $1 / 7$ & \\
\hline 20 & UE-25 UZN $\# 28$ & 1.73 & $1 / 7$ & $* *$ & 56 & USW UZ-N57 & $\mathrm{M}^{*}$ & & & 92 & UE-25 WT-18 & 1.10 & $1 / 8$ & \\
\hline 21 & UE-25 UZN \#29 & 1.25 & $1 / 7$ & & 57 & USW UZ-N64 & NI & & & 93 & USW H-5 & .93 & $1 / 8$ & \\
\hline 22 & UE-25 UZN \#30 & 1.30 & $1 / 7$ & & 58 & USW UZ-N65 & .89 & $1 / 8$ & & 94 & HRF Wedge & & & Removed \\
\hline 23 & UE-25 UZN \#56 & 1.23 & $1 / 7$ & & 59 & USW UZ-N66 & 1.18 & $1 / 8$ & & 94 & HRF 4" Gage & .94 & $1 / 6$ & \\
\hline 24 & UE-25 UZN $\# 60$ & 1.25 & $1 / 7$ & & 60 & USW UZ-N67 & 1.30 & $1 / 8$ & & 94 & HRF 8" Gage & .94 & $1 / 6$ & \\
\hline 25 & UE-25 UZN \#85 & .74 & $1 / 14$ & & 60 & N67 4" Gage & NI & & & 95 & USW H-3 & .82 & $1 / 8$ & \\
\hline 26 & UE-25 UZN \#92 & 1.80 & $1 / 6$ & ** & 61 & USW UZ-N68 & 1.28 & $1 / 8$ & & 96 & Weather station 4 (old) & .91 & $1 / 8$ & \\
\hline 26 & N92 4" Gage & NI & & & 62 & USW UZ-N69 & 1.23 & $1 / 8$ & & 97 & Weather station 5 (Prow) & $\mathbf{M}^{*}$ & & \\
\hline
\end{tabular}




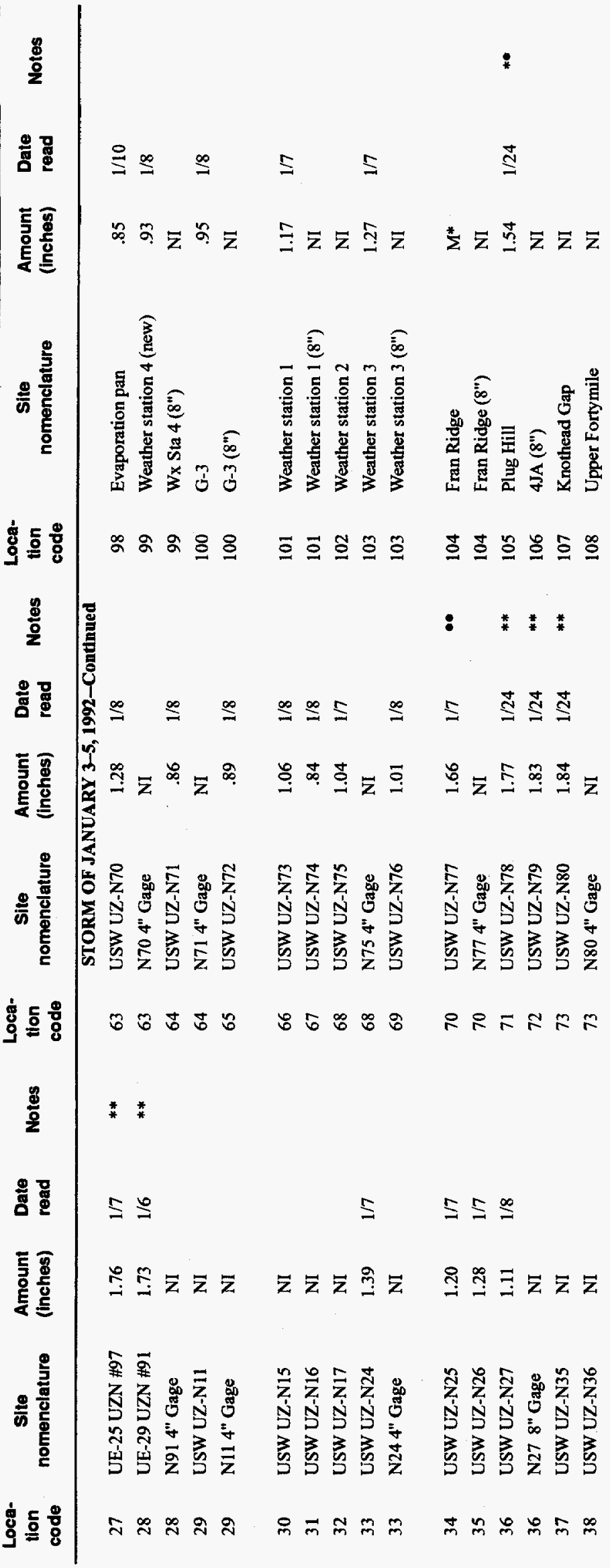


Table 2. Summary of precipitation data from the nonrecording gage network at Yucca Mountain, Nevada, for water years 1992 and 1993 --Continued

\begin{tabular}{|c|c|c|c|c|c|c|c|c|c|c|c|c|c|c|}
\hline $\begin{array}{l}\text { Loca- } \\
\text { tion } \\
\text { code }\end{array}$ & $\begin{array}{c}\text { Site } \\
\text { nomenelature }\end{array}$ & $\begin{array}{l}\text { Amount } \\
\text { (inches) }\end{array}$ & $\begin{array}{l}\text { Dale } \\
\text { read }\end{array}$ & Notes & $\begin{array}{l}\text { Loca- } \\
\text { tion } \\
\text { code }\end{array}$ & $\begin{array}{c}\text { Site } \\
\text { nomenclature }\end{array}$ & $\begin{array}{l}\text { Amount } \\
\text { (inches) }\end{array}$ & $\begin{array}{l}\text { Date } \\
\text { read }\end{array}$ & Notes & $\begin{array}{l}\text { Loca- } \\
\text { tion } \\
\text { code }\end{array}$ & $\begin{array}{c}\text { Site } \\
\text { nomenclature }\end{array}$ & $\begin{array}{l}\text { Amount } \\
\text { (inches) }\end{array}$ & $\begin{array}{l}\text { Date } \\
\text { read }\end{array}$ & Notes \\
\hline \multicolumn{15}{|c|}{ STORM OF FEB 6-7,9,1992 } \\
\hline 1 & UE-25 UZN \#1 & 1.27 & $2 / 10$ & & 39 & USW UZ-N37 & .46 & $2 / 10$ & & 74 & USW UZ-N81 & 1.26 & $2 / 10$ & \\
\hline 2 & UE-25 UZN \#2 & 1.37 & $2 / 10$ & @ & 39 & N37 4" Gage & NI & & & 75 & USW UZ-N82 & 1.16 & $2 / 10$ & \\
\hline 3 & UE-25 UZN \#3 & 1.22 & $2 / 10$ & & 40 & USW UZ-N40 & 1.32 & $2 / 10$ & & 75 & N82 4" Gage & 1.33 & $2 / 10$ & \\
\hline 4 & UE-25 UZN \#4 & 1.22 & $2 / 10$ & & 40 & N40 4" Gage & NI & & & 76 & USW UZ-N83 & 1.27 & $2 / 10$ & \\
\hline 4 & N4 4" Gage & NI & & & 41 & USW UZ-N41 & 1.37 & $2 / 10$ & & 77 & USW UZ-N84 & 1.24 & $2 / 10$ & \\
\hline 5 & UE-25 UZN \#5 & 1.22 & $2 / 10$ & & 42 & USW UZ-N42 & 1.37 & $2 / 10$ & & 78 & USW UZ-N86 & 1.32 & $2 / 10$ & \\
\hline 6 & UE-25 UZN \#6 & 1.25 & $2 / 10$ & & 43 & USW UZ-N43 & 1.30 & $2 / 10$ & & 79 & USW UZ-N87 & 1.25 & $2 / 10$ & \\
\hline 7 & UE-25 UZN \#7 & 1.25 & $2 / 10$ & & 43 & N43 4" Gage & NI & & & 80 & USW UZ-N88 & 1.23 & $2 / 10$ & \\
\hline 8 & UE-25 UZN \#8 & 1.22 & $2 / 10$ & & 44 & USW UZ-N44 & 1.35 & $2 / 10$ & & 81 & USW UZ-N89 & 1.22 & $2 / 10$ & \\
\hline 9 & UE-25 UZN \#9 & 1.22 & $2 / 10$ & & 45 & USW UZ-N45 & 1.32 & $2 / 10$ & & 82 & USW UZ-N90 & 1.23 & $2 / 10$ & \\
\hline 10 & UE-25 UZN \#10 & 1.30 & $2 / 10$ & & 46 & USW UZ-N46 & 1.59 & $2 / 10$ & (a) & 82 & N90 4" Gage & 1.41 & $2 / 10$ & \\
\hline 10 & N10 4" Gage & NI & & & 47 & USW UZ-N47 & 1.51 & $2 / 10$ & & 83 & USW UZ-N93 & 1.35 & $2 / 10$ & \\
\hline 11 & UE-25 UZN \#12 & 1.25 & $2 / 10$ & & 48 & USW UZ-N48 & 1.35 & $2 / 10$ & & 84 & USW UZ-N94 & 1.29 & $2 / 10$ & \\
\hline 12 & UE-25 UZN \#13 & 1.34 & $2 / 10$ & @ & & USW UZ-N49 & 1.25 & $2 / 10$ & & 85 & USW UZ-N95 & 1.27 & $2 / 10$ & \\
\hline 13 & UE-25 UZN \#14 & 1.17 & $2 / 10$ & & 49 & USW UZ-N50 & 1.22 & $2 / 10$ & & 86 & USW UZ-N96 & 1.29 & $2 / 10$ & \\
\hline 14 & UE-25 UZN \#18 & 1.27 & $2 / 10$ & & 50 & N50 4" Gage & NI & & & 87 & USW UZ-N98 & 1.39 & $2 / 10$ & \\
\hline 15 & UE-25 UZN \#19 & 1.22 & $2 / 10$ & & 50 & USW UZ-N51 & 1.22 & $2 / 10$ & & 87 & N98 4" Gage & NI & & \\
\hline 16 & UE-25 UZN \#20 & 1.21 & $2 / 10$ & & 51 & USW UZ-N52 & 1.14 & $2 / 10$ & & 88 & USW UZ-13 & 1.00 & $2 / 10$ & \\
\hline 17 & UE-25 UZN \#2I & 1.22 & $2 / 10$ & & 52 & USW UZ-N53 & 1.27 & $2 / 10$ & & 88 & UZ-13 4" Gage & NI & & \\
\hline 17 & N21 4" Gage & NI & & & 53 & USW UZ-N54 & $\mathbf{M}$ & & & 89 & USW GA-1 & 1.61 & $2 / 10$ & $@$ \\
\hline 18 & UE-25 UZN \#22 & 1.12 & $2 / 10$ & & 54 & N54 4" Gage & NI & & & 90 & USW G-2 & 1.58 & $2 / 10$ & $@$ \\
\hline 19 & UE-25 UZN \#23 & 1.18 & $2 / 10$ & & 54 & USW UZ-N55 & 1.20 & $2 / 10$ & & 91 & UE-25 WT-4 & 1.16 & $2 / 10$ & \\
\hline 20 & UE-25 UZN \#28 & 1.20 & $2 / 10$ & & 55 & USW UZ-N57 & 2.97 & $2 / 10$ & ** & 92 & UE-25 WT-18 & 1.18 & $2 / 10$ & $@$ \\
\hline 21 & UE-25 UZN $\# 29$ & 1.15 & $2 / 10$ & & 56 & USW UZ-N64 & $\mathrm{NI}$ & & & 93 & USW H-5 & 1.20 & $2 / 10$ & \\
\hline 22 & UE-25 UZN \#30 & 1.16 & $2 / 10$ & & 57 & USW UZ-N65 & .90 & $2 / 10$ & & 94 & HRF Wedge & & & Removed \\
\hline 23 & UE-25 UZN \#56 & 1.21 & $2 / 10$ & & 58 & USW UZ-N66 & 1.20 & $2 / 10$ & & 94 & HRF 4" Gage & .83 & $2 / 10$ & \\
\hline 24 & UE-25 UZN $\# 60$ & 1.20 & $2 / 10$ & & 59 & USW UZ-N67 & 1.21 & $2 / 10$ & & 94 & HRF 8" Gage & .84 & $2 / 10$ & \\
\hline 25 & UE-25 UZN \#85 & .89 & $2 / 10$ & $@$ & 60 & N67 4" Gage & NI & & & 95 & USW H-3 & 1.50 & $2 / 10$ & \\
\hline 26 & UE-25 UZN $\# 92$ & .95 & $2 / 10$ & & 61 & USW UZ-N68 & 1.22 & $2 / 10$ & & 96 & Weather station 4 (old) & 1.06 & $2 / 10$ & \\
\hline 26 & N92 4" Gage & NI & & & 62 & USW UZ-N69 & 1.19 & $2 / 10$ & & 97 & Weather station 5 (Prow) & $\mathbf{M}^{*}$ & & \\
\hline
\end{tabular}


N Table 2. Summary of precipitation data from the nonrecording gage network at Yucca Mountain, Nevada, for water years 1992 and $1993-$-Continued 
Table 2. Summary of precipitation data from the nonrecording gage network at Yucca Mountain, Nevada, for water years 1992 and $1993--$ Continued

\begin{tabular}{|c|c|c|c|c|c|c|c|c|c|c|c|c|c|c|}
\hline $\begin{array}{l}\text { Loca- } \\
\text { tlon } \\
\text { code }\end{array}$ & $\begin{array}{c}\text { Site } \\
\text { nomenclature }\end{array}$ & $\begin{array}{l}\text { Amount } \\
\text { (inches) }\end{array}$ & $\begin{array}{l}\text { Date } \\
\text { read }\end{array}$ & Notes & $\begin{array}{l}\text { Loca- } \\
\text { tlon } \\
\text { code }\end{array}$ & $\begin{array}{c}\text { Site } \\
\text { nomenclature }\end{array}$ & $\begin{array}{l}\text { Amount } \\
\text { (inches) }\end{array}$ & $\begin{array}{l}\text { Date } \\
\text { read }\end{array}$ & Notes & $\begin{array}{l}\text { Loce- } \\
\text { tlon } \\
\text { codo }\end{array}$ & $\begin{array}{c}\text { Site } \\
\text { nomenclature }\end{array}$ & $\begin{array}{l}\text { Amount } \\
\text { (inches) }\end{array}$ & $\begin{array}{l}\text { Date } \\
\text { read }\end{array}$ & Notes \\
\hline \multicolumn{15}{|c|}{ STORM OF FEBRUARY 10-12, 1992} \\
\hline 1 & UE-25 UZN \#1 & 1.26 & $2 / 13$ & & 39 & USW UZ-N37 & $\mathrm{M}^{*}$ & & & 74 & USW UZ-N81 & 1.12 & $2 / 13$ & \\
\hline 2 & UE-25 UZN \#2 & 1.43 & $2 / 13$ & & 39 & N37 4" Gage & NI & & & 75 & USW UZ-N82 & 1.01 & $2 / 13$ & \\
\hline 3 & UE-25 UZN \#3 & 1.33 & $2 / 13$ & & 40 & USW UZ-N40 & $\mathrm{M}^{*}$ & & & 75 & N82 4" Gage & 1.16 & $2 / 13$ & \\
\hline 4 & UE-25 UZN \#4 & 1.33 & $2 / 13$ & & 40 & N40 4" Gage & $\mathrm{NI}$ & & & 76 & USW UZ-N83 & 1.18 & $2 / 13$ & \\
\hline 4 & N4 4" Gage & NI & & & 41 & USW UZ-N4I & $\mathrm{M}^{*}$ & & & 77 & USW UZ-N84 & 1.11 & $2 / 13$ & \\
\hline 5 & UE-25 UZN \#5 & 1.28 & $2 / 13$ & & 42 & USW UZ-N42 & $\mathbf{M}^{*}$ & & & 78 & USW UZ-N86 & 1.18 & $2 / 13$ & \\
\hline 6 & UE-25 UZN \#6 & 1.30 & $2 / 13$ & & 43 & USW UZ-N43 & $\mathbf{M}^{*}$ & & & 79 & USW UZ-N87 & 1.10 & $2 / 13$ & \\
\hline 7 & UE-25 UZN \#7 & 1.30 & $2 / 13$ & & 43 & N43 4" Gage & $\mathrm{NI}$ & & & 80 & USW UZ-N88 & 1.22 & $2 / 13$ & \\
\hline 8 & UE-25 UZN \#8 & 1.38 & $2 / 13$ & & 44 & USW UZ-N44 & $\mathrm{M}^{*}$ & & & 81 & USW UZ-N89 & 1.15 & $2 / 13$ & \\
\hline 9 & UE-25 UZN \#9 & 1.36 & $2 / 13$ & & 45 & USW UZ-N45 & $\mathbf{M}^{*}$ & & & 82 & USW UZ-N90 & 1.12 & $2 / 13$ & \\
\hline 10 & UE-25 UZN \#10 & 1.25 & $2 / 13$ & & 46 & USW UZ-N46 & $\mathbf{M}^{*}$ & & & 82 & N90 4" Gage & 1.26 & $2 / 13$ & \\
\hline 10 & N10 4" Gage & NI & & & 47 & USW UZ-N47 & $\mathbf{M}^{*}$ & & & 83 & USW UZ-N93 & $\mathbf{M}^{*}$ & & \\
\hline 11 & UE-25 UZN \#12 & 1.30 & $2 / 13$ & & 48 & USW UZ-N48 & $\mathbf{M}^{*}$ & & & 84 & USW UZ-N94 & $\mathrm{M}^{*}$ & & \\
\hline 12 & UE-25 UZN \#13 & 1.50 & $2 / 13$ & & 49 & USW UZ-N49 & $\mathbf{M}^{*}$ & & & 85 & USW UZ-N95 & $\mathbf{M}^{*}$ & & \\
\hline 13 & UE-25 UZN \#14 & 1.28 & $2 / 13$ & & 50 & USW UZ-N50 & $\mathbf{M}^{*}$ & & & 86 & USW UZ-N96 & $\mathbf{M}^{*}$ & & \\
\hline 14 & UE-25 UZN \#18 & $\mathbf{M}^{*}$ & & & 50 & N50 4" Gage & NI & & & 87 & USW UZ-N98 & $\mathrm{M}^{*}$ & & \\
\hline 15 & UE-25 UZN \#19 & $\mathrm{M}^{*}$ & & & 51 & USW UZ-N51 & $\mathrm{M}^{*}$ & & & 87 & N98 4" Gage & NI & & \\
\hline 16 & UE-25 UZN \#20 & $\mathbf{M}^{*}$ & & & 52 & USW UZ-N52 & $\mathbf{M}^{*}$ & & & 88 & USW UZ-13 & $\mathrm{M}^{*}$ & & \\
\hline 17 & UE-25 UZN \#21 & $\mathbf{M}^{*}$ & & & 53 & USW UZ-N53 & $\mathbf{M}^{*}$ & & & 88 & UZ-13 4" Gage & NI & & \\
\hline 17 & N21 4" Gage & $\mathrm{NI}$ & & & 54 & USW UZ-N54 & $\mathbf{M}$ & & & 89 & USW GA-1 & $\mathbf{M}^{*}$ & & \\
\hline 18 & UE-25 UZN \#22 & $\mathbf{M}^{*}$ & & & 54 & N54 4" Gage & NI & & & 90 & USW G-2 & $\mathrm{M}^{*}$ & & \\
\hline 19 & UE-25 UZN $\# 23$ & $\mathbf{M}^{*}$ & & & 55 & USW UZ-N55 & $\mathbf{M}^{*}$ & & & 91 & UE-25 WT-4 & 1.27 & & \\
\hline 20 & UE-25 UZN \#28 & $\mathbf{M}^{*}$ & & & 56 & USW UZ-N57 & $\mathbf{M}^{*}$ & & & 92 & UE-25 WT-18 & $\mathbf{M}^{*}$ & & \\
\hline 21 & UE-25 UZN \#29 & $\mathbf{M}^{*}$ & & & 57 & USW UZ-N64 & NI & & & 93 & USW H-5 & $\mathrm{M}^{*}$ & & \\
\hline 22 & UE-25 UZN \#30 & $\mathrm{M}^{*}$ & & & 58 & USW UZ-N65 & $\mathrm{M}^{*}$ & & & 94 & HRF Wedge & & & Removed \\
\hline 23 & UE-25 UZN \#56 & $\mathrm{M}^{*}$ & & & 59 & USW UZ-N66 & $\mathbf{M}^{*}$ & & & 94 & HRF 4" Gage & 1.07 & $2 / 13$ & \\
\hline 24 & UE-25 UZN $\# 60$ & $\mathrm{M}^{*}$ & & & 60 & USW UZ-N67 & $\mathbf{M}^{*}$ & & & 94 & HRF 8" Gage & 1.09 & $2 / 13$ & \\
\hline 25 & UE-25 UZN \#85 & $\mathrm{M}^{*}$ & & & 60 & N67 4" Gage & NI & & & 95 & USW H-3 & $\mathbf{M}^{*}$ & & \\
\hline 26 & UE-25 UZN $\# 92$ & $\mathbf{M}^{*}$ & & & 61 & USW UZ-N68 & $\mathrm{M}^{*}$ & & & 96 & Weather station 4 (old) & $\mathrm{M}^{*}$ & & \\
\hline 26 & N92 4" Gage & NI & & & 62 & USW UZ-N69 & $\mathrm{M}^{*}$ & & & 97 & Weather station 5 (Prow) & $\mathrm{M}^{*}$ & & \\
\hline
\end{tabular}


Table 2. Summary of precipitation data from the nonrecording gage network at Yucca Mountain, Nevada, for water years 1992 and 1993--Continued

\begin{tabular}{|c|c|c|c|c|c|c|c|c|c|c|c|c|c|c|}
\hline $\begin{array}{l}\text { Loca- } \\
\text { tlon } \\
\text { code }\end{array}$ & $\begin{array}{c}\text { Site } \\
\text { nomenclature }\end{array}$ & $\begin{array}{l}\text { Amount } \\
\text { (inches) }\end{array}$ & $\begin{array}{l}\text { Date } \\
\text { read }\end{array}$ & Notes & $\begin{array}{l}\text { Loca- } \\
\text { tion } \\
\text { code }\end{array}$ & $\begin{array}{c}\text { Site } \\
\text { nomenclature }\end{array}$ & $\begin{array}{l}\text { Amount } \\
\text { (inches) }\end{array}$ & $\begin{array}{l}\text { Date } \\
\text { read }\end{array}$ & Notes & $\begin{array}{l}\text { Loca- } \\
\text { tion } \\
\text { code }\end{array}$ & $\begin{array}{c}\text { Site } \\
\text { nomenclature }\end{array}$ & $\begin{array}{l}\text { Amount } \\
\text { (inches) }\end{array}$ & $\begin{array}{l}\text { Date } \\
\text { read }\end{array}$ & Noles \\
\hline \multicolumn{15}{|c|}{ STORM OF FEBRUARY 10-12, 1992-Conthued } \\
\hline 27 & UE-25 UZN \#97 & $\mathrm{M}^{*}$ & & & 63 & USW UZ-N70 & $\mathrm{M}^{*}$ & & & 98 & Evaporation pan & $\mathbf{M}^{*}$ & & \\
\hline 28 & UE-29 UZN \#1 1 & $\mathbf{M}^{*}$ & & & 63 & N70 4" Gage & NI & & & 99 & Weather station 4 (new) & $\mathbf{M}^{*}$ & & \\
\hline 28 & N91 4" Gage & NI & & & 64 & USW UZ-N71 & $\mathrm{M}^{*}$ & & & 99 & Weather station $4\left(8^{\prime \prime}\right)$ & NI & & \\
\hline 29 & USW UZ-N11 & NI & & & 64 & N71 4" Gage & NI & & & 100 & G-3 & $\mathrm{M}^{*}$ & & \\
\hline 29 & N11 4" Gage & NI & & & 65 & USW UZ-N72 & $\mathrm{M}^{*}$ & & & 100 & G-3 8" Gage & NI & & \\
\hline 30 & USW UZ-N15 & NI & & & 66 & USW UZ-N73 & $\mathrm{M}^{*}$ & & & 101 & Weather station 1 & $\mathbf{M}^{*}$ & & \\
\hline 31 & USW UZ-N16 & NI & & & 67 & USW UZ-N74 & $\mathrm{M}^{*}$ & & & 101 & Weather station $1(8 ")$ & NI & & \\
\hline 32 & USW UZ-N17 & NI & & & 68 & USW UZ-N75 & $\mathbf{M}^{*}$ & & & 102 & Weather station 2 & NI & & \\
\hline 33 & USW UZ-N24 & $\mathrm{M}^{*}$ & & & 68 & N75 4" Gage & NI & & & 103 & Weather station 3 & $\mathrm{M}^{*}$ & & \\
\hline 33 & N24 4" Gage & NI & & & 69 . & USW UZ-N76 & $\mathrm{M}^{*}$ & & & 103 & Weather station $3\left(8^{\prime \prime}\right)$ & NI & & \\
\hline 34 & USW UZ-N25 & $\mathrm{M}^{*}$ & & & 70 & USW UZ-N77 & .93 & $2 / 13$ & & 104 & Fran Ridge & $\mathrm{M}^{*}$ & & \\
\hline 35 & USW UZ-N26 & $\mathrm{M}^{*}$ & & & 70 & N77 4" Gage & 1.11 & $2 / 13$ & & 104 & Fran Ridge (8") & NI & & \\
\hline 36 & USW UZ-N27 & $\mathrm{M}^{*}$ & & & 71 & USW UZ-N78 & 1.05 & $2 / 13$ & & 105 & Plug Hill & .80 & $2 / 13$ & \\
\hline 36 & N27 8" Gage & $\mathrm{NI}$ & & & 72 & USW UZ-N79 & 1.15 & $2 / 13$ & & 106 & 4JA (8") & $\mathrm{NI}$ & & \\
\hline 37 & USW UZ-N35 & NI & & & 73 & USW UZ-N80 & 1.25 & $2 / 13$ & & 107 & Knothead Gap & NI & & \\
\hline 38 & USW UZ-N36 & NI & & & 73 & N80 4" Gage & 1.41 & $2 / 13$ & & 108 & Upper Forty Mile & NI & & \\
\hline
\end{tabular}


Table 2. Summary of precipitation data from the nonrecording gage network at Yucca Mountain, Nevada, for water years 1992 and $1993--$ Continued

\begin{tabular}{|c|c|c|c|c|c|c|c|c|c|c|c|c|c|c|}
\hline $\begin{array}{l}\text { Loca- } \\
\text { ilon } \\
\text { code }\end{array}$ & $\begin{array}{c}\text { Site } \\
\text { nomenclature }\end{array}$ & $\begin{array}{l}\text { Amount } \\
\text { (Inches) }\end{array}$ & $\begin{array}{l}\text { Date } \\
\text { read }\end{array}$ & Notes & $\begin{array}{l}\text { Loca- } \\
\text { tlon } \\
\text { code }\end{array}$ & $\begin{array}{c}\text { Site } \\
\text { nomenclature }\end{array}$ & $\begin{array}{l}\text { Amount } \\
\text { (inches) }\end{array}$ & $\begin{array}{l}\text { Date } \\
\text { read }\end{array}$ & Notes & $\begin{array}{l}\text { Loca- } \\
\text { tion } \\
\text { code }\end{array}$ & $\begin{array}{c}\text { Sile } \\
\text { nomenclature }\end{array}$ & $\begin{array}{l}\text { Amount } \\
\text { (inches) }\end{array}$ & $\begin{array}{l}\text { Date } \\
\text { read }\end{array}$ & Notes \\
\hline \multicolumn{15}{|c|}{ STORM OF FEBRUARY 13,1992} \\
\hline 1 & UE-25 UZN \#1 & .27 & $2 / 14$ & & 39 & USW UZ-N37 & 1.48 & $2 / 14$ & ** & 74 & USW UZ-N81 & .27 & $2 / 14$ & \\
\hline 2 & UE-25 UZN \#2 & .27 & $2 / 14$ & & 39 & N37 4" Gage & NI & & & 75 & USW UZ-N82 & .18 & $2 / 14$ & \\
\hline 3 & UE-25 UZN \#3 & .22 & $2 / 14$ & & 40 & USW UZ-N40 & 1.58 & $2 / 14$ & $* *$ & 75 & N82 4" Gage & .20 & $2 / 14$ & \\
\hline 4 & UE-25 UZN \#4 & .27 & $2 / 14$ & & 40 & N40 4" Gage & NI & & & 76 & USW UZ-N83 & .25 & $2 / 14$ & \\
\hline 4 & N44" Gage & NI & & & 41 & USW UZ-N41 & 1.63 & $2 / 14$ & ** & 77 & USW UZ-N84 & .23 & $2 / 14$ & \\
\hline 5 & UE-25 UZN \#5 & .27 & $2 / 14$ & & 42 & USW UZ-N42 & 1.65 & $2 / 14$ & *** & 78 & USW UZ-N86 & .15 & $2 / 14$ & \\
\hline 6 & UE-25 UZN \#6 & .25 & $2 / 14$ & & 43 & USW UZ-N43 & 1.65 & $2 / 14$ & ** & 79 & USW UZ-N87 & .17 & $2 / 14$ & \\
\hline 7 & UE-25 UZN \#7 & .23 & $2 / 14$ & & 43 & N43 4" Gage & $\mathrm{NI}$ & & & 80 & USW UZ-N88 & .23 & $2 / 14$ & \\
\hline 8 & UE-25 UZN \#8 & .22 & $2 / 14$ & & 44 & USW UZ-N44 & 1.56 & $2 / 14$ & ** & 81 & USW UZ-N89 & .18 & $2 / 14$ & \\
\hline 9 & UE-25 UZN \#9 & .22 & $2 / 14$ & & 45 & USW UZ-N45 & 1.63 & $2 / 14$ & ** & 82 & USW UZ-N90 & .17 & $2 / 14$ & \\
\hline 10 & UE-25 UZN \#10 & .30 & $2 / 14$ & & 46 & USW UZ-N46 & 1.89 & $2 / 14$ & $* *$ & 82 & N90 4" Gage & .20 & $2 / 14$ & \\
\hline 10 & N10 4" Gage & NI & & & 47 & USW UZ-N47 & 1.82 & $2 / 14$ & ** & 83 & USW UZ-N93 & 1.52 & $2 / 14$ & \\
\hline 11 & UE-25 UZN \#12 & .27 & $2 / 14$ & & 48 & USW UZ-N48 & 1.70 & $2 / 14$ & $* *$ & 84 & USW UZ-N94 & 1.53 & $2 / 14$ & $* *$ \\
\hline 12 & UE-25 UZN \#13 & .24 & $2 / 14$ & & 49 & USW UZ-N49 & 1.57 & $2 / 14$ & $* *$ & 85 & USW UZ-N95 & 1.52 & $2 / 14$ & $* *$ \\
\hline 13 & UE-25 UZN \#14 & .22 & $2 / 14$ & & 50 & USW UZ-N50 & 1.70 & $2 / 14$ & $* *$ & 86 & USW UZ-N96 & 1.28 & $2 / 14$ & $* *$ \\
\hline 14 & UE-25 UZN \#18 & 1.48 & $2 / 14$ & $* *$ & 50 & N50 4" Gage & NI & & & 87 & USW UZ-N98 & 1.86 & $2 / 14$ & $* *$ \\
\hline 15 & UE-25 UZN \#19 & 1.78 & $2 / 14$ & $* *$ & 51 & USW UZ-N51 & 1.65 & $2 / 14$ & $* *$ & 87 & N98 4" Gage & NI & & \\
\hline 16 & UE-25 UZN \#20 & 1.70 & $2 / 14$ & $* *$ & 52 & USW UZ-N52 & 1.63 & $2 / 14$ & $* *$ & 88 & USW UZ 13 & 1.20 & $2 / 14$ & $* *$ \\
\hline 17 & UE-25 UZN \#21 & 1.73 & $2 / 14$ & $* *$ & 53 & USW UZ-N53 & 1.43 & $2 / 14$ & $* *$ & 88 & UZ-13 4" Gage & NI & & \\
\hline 17 & N21 4" Gage & NI & & & 54 & USW UZ-N54 & $\mathbf{M}$ & & & 89 & USW GA-1 & 1.94 & $2 / 14$ & $* *$ \\
\hline 18 & UE-25 UZN \#22 & 1.75 & $2 / 14$ & $* *$ & 54 & N54 4" Gage & NI & & & 90 & USW G-2 & 2.02 & $2 / 14$ & *** \\
\hline 19 & UE-25 UZN \#23 & 1.60 & $2 / 14$ & $* *$ & 55 & USW UZ-N5S & 1.35 & $2 / 14$ & ** & 91 & UE25 WT-4 & .22 & $2 / 14$ & \\
\hline 20 & UE-25 UZN \#28 & 1.70 & $2 / 14$ & $* *$ & 56 & USW UZ-N57 & 1.47 & $2 / 14$ & $* *$ & 92 & UE2S WT-18 & 1.56 & $2 / 14$ & $* *$ \\
\hline 21 & UE-25 UZN $\# 29$ & 1.60 & $2 / 14$ & $* *$ & 57 & USW UZ-N64 & NI & & & 93 & USW H-S & 1.74 & $2 / 14$ & $* *$ \\
\hline 22 & UE-25 UZN \#30 & 1.70 & $2 / 14$ & $* *$ & 58 & USW UZ-N65 & 1.25 & $2 / 14$ & $* *$ & 94 & HRF Wedge & & & Removed \\
\hline
\end{tabular}


Table 2. Summary of precipitation data from the nonrecording gage network at Yucca Mountain. Nevada, for water years 1992 and $1993--$ Continued

\begin{tabular}{|c|c|c|c|c|c|c|c|c|c|c|c|c|c|c|}
\hline $\begin{array}{l}\text { Loca- } \\
\text { tlon } \\
\text { code }\end{array}$ & $\begin{array}{c}\text { Site } \\
\text { nomenclature }\end{array}$ & $\begin{array}{l}\text { Amount } \\
\text { (Inches) }\end{array}$ & $\begin{array}{l}\text { Date } \\
\text { read }\end{array}$ & Notes & $\begin{array}{l}\text { Loca- } \\
\text { tion } \\
\text { code }\end{array}$ & $\begin{array}{c}\text { Site } \\
\text { nomenclature }\end{array}$ & $\begin{array}{l}\text { Amount } \\
\text { (inches) }\end{array}$ & $\begin{array}{l}\text { Date } \\
\text { read }\end{array}$ & Noles & $\begin{array}{l}\text { Loca- } \\
\text { tion } \\
\text { code }\end{array}$ & $\begin{array}{c}\text { Sile } \\
\text { nomenclature }\end{array}$ & $\begin{array}{l}\text { Amount } \\
\text { (inchos) }\end{array}$ & $\begin{array}{l}\text { Date } \\
\text { read }\end{array}$ & Notes \\
\hline \multicolumn{15}{|c|}{ STORM OF FEBRUARY 13, 1992-Contlnued } \\
\hline 23 & UE-25 UZN \#56 & 1.54 & $2 / 14$ & ** & 59 & USW UZ-N66 & 1.48 & $2 / 14$ & $* *$ & 94 & HRF 4" Gage & .21 & $2 / 14$ & \\
\hline 25 & UE-25 UZN \#85 & $\mathbf{M}^{*}$ & & & 60 & N67 4" Gage & NI & & & 95 & USW H-3 & 1.33 & $2 / 14$ & $* *$ \\
\hline 26 & UE-25 UZN \#92 & 1.70 & $2 / 14$ & $* *$ & 61 & USW UZ-N68 & 1.41 & $2 / 14$ & $* *$ & 96 & Wea station & 1.40 & $2 / 14$ & ** \\
\hline 26 & N92 4" Gage & NI & & & 62 & USW UZ-N69 & 1.41 & $2 / 14$ & ** & 97 & Wea station & $\mathbf{M}^{*}$ & & \\
\hline 28 & UE-29 UZN \#91 & $\mathbf{M}^{*}$ & & & 63 & N70 4" Gage & NI & & & 99 & Wea station & 1.44 & $2 / 14$ & ** \\
\hline 28 & N91 4" Gage & NI & & & 64 & USW UZ-N71 & 1.40 & $2 / 14$ & ** & 99 & Wea station & NI & & \\
\hline 29 & USW UZ-N11 & NI & & & 64 & N71 4" Gage & NI & & & 100 & G-3 & 1.30 & $2 / 14$ & $* *$ \\
\hline 29 & N11 4" Gage & NI & & & 65 & USW UZ-N72 & 1.45 & $2 / 14$ & $* *$ & 100 & G-3 8" Gage & NI & & \\
\hline 30 & USW UZ-N15 & NI & & & 66 & USW UZ-N73 & 1.55 & $2 / 14$ & $* *$ & 101 & Wea station & $\mathbf{M}^{*}$ & & \\
\hline 31 & USW UZ-N16 & NI & & & 67 & USW UZ-N74 & 1.42 & $2 / 14$ & ** & 101 & Wea station & NI & & \\
\hline 32 & USW UZ-N17 & $\mathrm{NI}$ & & & 68 & USW UZ-N75 & 1.50 & $2 / 14$ & $* *$ & 102 & Wea station & NI & & \\
\hline 33 & USW UZ-N24 & 1.85 & $2 / 14$ & $* *$ & 68 & N75 4" Gage & NI & & & 103 & Wea station & 1.67 & $2 / 21$ & $* *$ \\
\hline 33 & N24 4" Gage & $\mathrm{NI}$ & & & 69 & USW UZ-N76 & 1.50 & $2 / 14$ & $* *$ & 103 & Wea station & $\mathrm{NI}$ & & \\
\hline 34 & USW UZ-N25 & 1.69 & $2 / 14$ & $* *$ & 70 & USW UZ-N77 & .18 & $2 / 14$ & & 104 & Fran Ridge & 3.85 & $2 / 14$ & $* *$ \\
\hline 35 & USW UZ-N26 & 1.75 & $2 / 14$ & $* *$ & 70 & N77 4" Gage & .20 & $2 / 14$ & & 104 & Fran Ridge (8") & $\mathrm{NI}$ & & \\
\hline 36 & USW UZ-N27 & 1.80 & $2 / 14$ & $* *$ & 71 & USW UZ-N78 & .25 & $2 / 14$ & & 105 & Plug Hill & .15 & $2 / 14$ & \\
\hline 36 & N27 8" Gage & & NI & & 72 & USW UZ-N79 & .20 & $2 / 14$ & & 106 & 4JA (8") & NI & & \\
\hline 37 & USW UZ-N35 & NI & & & 73 & USW UZ-N80 & .27 & $2 / 14$ & & 107 & Knothead Gap & NI & & \\
\hline 38 & USW UZ-N36 & NI & & & 73 & N80 4" Gage & .27 & $2 / 14$ & & 108 & Upper Forty Mile & $\mathrm{NI}$ & & \\
\hline
\end{tabular}


Table 2. Summary of precipitation data from the nonrecording gage network at Yucca Mountain, Nevada, for water years 1992 and 1993 --Continued

\begin{tabular}{|c|c|c|c|c|c|c|c|c|c|c|c|c|c|c|}
\hline $\begin{array}{l}\text { Loca- } \\
\text { tion } \\
\text { code }\end{array}$ & $\begin{array}{c}\text { Site } \\
\text { nomenclature }\end{array}$ & $\begin{array}{l}\text { Amount } \\
\text { (Inches) }\end{array}$ & $\begin{array}{l}\text { Date } \\
\text { read }\end{array}$ & Notes & $\begin{array}{l}\text { Loca- } \\
\text { tion } \\
\text { code }\end{array}$ & $\begin{array}{c}\text { Site } \\
\text { nomenclature }\end{array}$ & $\begin{array}{l}\text { Amount } \\
\text { (inches) }\end{array}$ & $\begin{array}{l}\text { Date } \\
\text { read }\end{array}$ & Notes & $\begin{array}{l}\text { Loca- } \\
\text { tlon } \\
\text { code }\end{array}$ & $\begin{array}{c}\text { Site } \\
\text { nomenclature }\end{array}$ & $\begin{array}{l}\text { Amount } \\
\text { (inches) }\end{array}$ & $\begin{array}{l}\text { Date } \\
\text { read }\end{array}$ & Notes \\
\hline \multicolumn{15}{|c|}{ STORM OF FEBRUARY 15-18, 1992} \\
\hline 1 & UE-25 UZN \#1 & .40 & $2 / 19$ & & 39 & USW UZ-N37 & .50 & $2 / 19$ & & 74 & USW UZ-N81 & .30 & $2 / 19$ & \\
\hline 2 & UE-25 UZN \#2 & .27 & $2 / 19$ & & 39 & N37 4" Gage & $\mathrm{NI}$ & & & 75 & USW UZ-N82 & .37 & $2 / 19$ & \\
\hline 3 & UE-25 UZN \#3 & .48 & $2 / 19$ & & 40 & USW UZ-N40 & .45 & $2 / 19$ & & 75 & N82 4" Gage & .41 & $2 / 19$ & \\
\hline 4 & UE-25 UZN \#4 & .48 & $2 / 19$ & & 40 & N40 4" Gage & NI & & & 76 & USW UZ-N83 & .60 & $2 / 19$ & \\
\hline 4 & N4 4" Gage & NI & & & 41 & USW UZ-N41 & .50 & $2 / 19$ & & 77 & USW UZ-N84 & .44 & $2 / 19$ & \\
\hline 5 & UE-25 UZN \#5 & .48 & $2 / 19$ & & 42 & USW UZ-N42 & .48 & $2 / 19$ & & 78 & USW UZ-N86 & .55 & $2 / 19$ & \\
\hline 6 & UE-25 UZN \#6 & .50 & $2 / 19$ & & 43 & USW UZ-N43 & .45 & $2 / 19$ & & 79 & USW UZ-N87 & .49 & $2 / 19$ & \\
\hline 7 & UE-25 UZN \#7 & .53 & $2 / 19$ & & 43 & N43 4" Gage & $\mathrm{NI}$ & & & 80 & USW UZ-N88 & .58 & $2 / 19$ & \\
\hline 8 & UE-25 UZN \#8 & .51 & $2 / 19$ & & 44 & USW UZ-N44 & .39 & $2 / 19$ & & 81 & USW UZ-N89 & .49 & $2 / 19$ & \\
\hline 9 & UE-25 UZN \#9 & .55 & $2 / 19$ & & 45 & USW UZ-N45 & .46 & $2 / 19$ & & 82 & USW UZ-N90 & .48 & $2 / 19$ & \\
\hline 10 & UE-25 UZN \#10 & .45 & $2 / 19$ & & 46 & USW UZ-N46 & .44 & $2 / 19$ & & 82 & N90 4" Gage & .51 & $2 / 19$ & \\
\hline 10 & N10 4" Gage & $\mathrm{NI}$ & & & 47 & USW UZ-N47 & .47 & $2 / 19$ & & 83 & USW UZ-N93 & $\mathbf{M}^{*}$ & & \\
\hline 11 & UE-25 UZN \#12 & .53 & $2 / 19$ & & 48 & USW UZ-N48 & .45 & $2 / 19$ & & 84 & USW UZ-N94 & $\mathbf{M}^{*}$ & & \\
\hline 12 & UE-25 UZN \#13 & .49 & $2 / 19$ & & 49 & USW UZ-N49 & .38 & $2 / 19$ & & 85 & USW UZ-N95 & $\mathbf{M}^{*}$ & & \\
\hline 13 & UE-25 UZN \#14 & .47 & $2 / 19$ & & 50 & USW UZ-N50 & .39 & $2 / 19$ & & 86 & USW UZ-N96 & $\mathrm{M}^{*}$ & & \\
\hline 14 & UE-25 UZN \#18 & .52 & $2 / 19$ & & 50 & N50 4" Gage & $\mathrm{NI}$ & & & 87 & USW UZ-N98 & .50 & $2 / 19$ & \\
\hline 15 & UE-25 UZN \#19 & .40 & $2 / 18$ & & 51 & USW UZ-N51 & .53 & $2 / 19$ & & 87 & N98 4" Gage & NI & & \\
\hline 16 & UE-25 UZN \#20 & .45 & $2 / 18$ & & 52 & USW UZ-N52 & .55 & $2 / 19$ & & 88 & USW UZ 13 & $\mathbf{M}^{*}$ & & \\
\hline 17 & UE-25 UZN \#21 & .44 & $2 / 18$ & & 53 & USW UZ-N53 & $\mathrm{M}^{*}$ & & & 88 & UZ-13 4" Gage & NI & & \\
\hline 17 & N21 4" Gage & NI & & & 54 & USW UZ-N54 & $\mathbf{M}$ & & & 89 & USW GA-1 & 1.94 & $2 / 19$ & \\
\hline 18 & UE-25 UZN \#22 & .44 & $2 / 18$ & & 54 & N54 4" Gage & $\mathrm{NI}$ & & & 90 & USW G-2 & $\mathbf{M}$ & $2 / 19$ & \\
\hline 19 & UE-25 UZN \#23 & .45 & $2 / 18$ & & 55 & USW UZ-N55 & .70 & $2 / 19$ & & 91 & UE25 WT-4 & .44 & $2 / 19$ & \\
\hline 20 & UE-25 UZN \#28 & .38 & $2 / 18$ & & 56 & USW UZ-N57 & .18 & $2 / 19$ & & 92 & UE25 WT-18 & $\mathbf{M}$ & & \\
\hline 21 & UE-25 UZN $\# 29$ & .39 & $2 / 18$ & & 57 & USW UZ-N64 & NI & & & 93 & USW H-5 & .35 & $2 / 19$ & \\
\hline 22 & UE-25 UZN \#30 & .30 & $2 / 18$ & & 58 & USW UZ-N65 & $\mathrm{M}^{*}$ & $2 / 19$ & & 94 & HRF Wedge & .06 & $2 / 18$ & Restored \\
\hline
\end{tabular}


N Table 2. Summary of precipitation data from the nonrecording gage network at Yucca Mountain, Nevada, for water years 1992 and $1993-$-Continued

\begin{tabular}{|c|c|c|c|c|c|c|c|c|c|c|c|c|c|c|}
\hline $\begin{array}{l}\text { Loca- } \\
\text { tion } \\
\text { code }\end{array}$ & $\begin{array}{c}\text { Site } \\
\text { nomenclature }\end{array}$ & $\begin{array}{l}\text { Amount } \\
\text { (inches) }\end{array}$ & $\begin{array}{l}\text { Date } \\
\text { read }\end{array}$ & Notes & $\begin{array}{l}\text { Loca- } \\
\text { tion } \\
\text { code }\end{array}$ & $\begin{array}{c}\text { Site } \\
\text { nomenclature }\end{array}$ & $\begin{array}{l}\text { Amount } \\
\text { (inches) }\end{array}$ & $\begin{array}{l}\text { Date } \\
\text { read }\end{array}$ & Notes & $\begin{array}{l}\text { Loca- } \\
\text { tion } \\
\text { code }\end{array}$ & $\begin{array}{c}\text { Site } \\
\text { nomenclature }\end{array}$ & $\begin{array}{l}\text { Amount } \\
\text { (inches) }\end{array}$ & $\begin{array}{l}\text { Date } \\
\text { read }\end{array}$ & Notes \\
\hline \multicolumn{15}{|c|}{ STORM OF FEBRUARY 15-18, 1992-Continued } \\
\hline 23 & UE-25 UZN \#56 & .35 & $2 / 19$ & & 59 & USW UZ-N66 & .30 & $2 / 19$ & & 94 & HRF 4" Gage & .08 & $2 / 18$ & \\
\hline 24 & UE-25 UZN \#60 & .30 & $2 / 19$ & & 60 & USW UZ-N67 & $\mathbf{M}^{*}$ & $2 / 19$ & & 94 & HRF 8" Gage & .06 & $2 / 18$ & \\
\hline 25 & UE-25 UZN \#85 & 1.29 & $2 / 19$ & EVAP $/ *$ & 60 & N67 4" Gage & NI & & & 95 & USW H-3 & $\mathbf{M}^{*}$ & & \\
\hline 26 & UE-25 UZN \#92 & .40 & $2 / 18$ & & 61 & USW UZ-N68 & $\mathrm{M}^{*}$ & $2 / 14$ & & 96 & Wea station & .35 & $2 / 19$ & \\
\hline 26 & N92 4" Gage & NI & & & 62 & USW UZ-N69 & $\mathbf{M}^{*}$ & $2 / 14$ & & 97 & Wea station & $\mathbf{M}^{*}$ & & \\
\hline 27 & UE-25 UZN $\$ 97$ & .30 & $2 / 18$ & & 63 & USW UZ-N70 & .50 & $2 / 19$ & & 98 & Evaporation & .03 & $2 / 19$ & \\
\hline 28 & UE-29 UZN \#91 & 2.65 & $2 / 18$ & $* *$ & 63 & N70 4" Gage & $\mathrm{NI}$ & & & 99 & Wea station & $\mathbf{M}^{*}$ & & \\
\hline 28 & N91 4" Gage & NI & & & 64 & USW UZ-N71 & .34 & $2 / 19$ & & 99 & Wea station & NI & & \\
\hline 29 & USW UZ-N11 & NI & & & 64 & N71 4" Gage & $\mathrm{NI}$ & & & 100 & G-3 & $\mathbf{M}^{*}$ & & \\
\hline 29 & N11 4" Gage & NI & & & 65 & USW UZ-N72 & .43 & $2 / 19$ & & 100 & G-3 8" Gage & $\mathrm{NI}$ & & \\
\hline 30 & USW UZ-N15 & NI & & & 66 & USW UZ-N73 & .45 & $2 / 19$ & & 101 & Wea station & 1.74 & $2 / 19$ & \\
\hline 31 & USW UZ-N16 & NI & & & 67 & USW UZ-N74 & .38 & $2 / 19$ & & 101 & Wea station & $\mathrm{NI}$ & & \\
\hline 32 & USW UZ-N17 & NI & & & 68 & USW UZ-N75 & .45 & $2 / 19$ & & 102 & Wea station & NI & & \\
\hline 33 & USW UZ-N24 & .49 & $2 / 19$ & & 68 & N75 4" Gage & NI & & & 103 & Wea station & .50 & $2 / 21$ & \\
\hline 33 & N24 4" Gage & NI & & & 69 & USW UZ-N76 & .41 & $2 / 19$ & & 103 & Wea station & $\mathrm{NI}$ & & \\
\hline 34 & USW UZ-N25 & .56 & $2 / 19$ & & 70 & USW UZ-N77 & .22 & $2 / 19$ & & 104 & Fran Ridge & $\mathbf{M}^{*}$ & & \\
\hline 35 & USW UZ-N26 & .54 & $2 / 19$ & & 70 & N77 4" Gage & .29 & $2 / 19$ & & 104 & Fran Ridge (8") & NI & & \\
\hline 36 & USW UZ-N27 & .37 & $2 / 19$ & & 71 & USW UZ-N78 & .26 & $2 / 19$ & & 105 & Plug Hill & .10 & $2 / 19$ & \\
\hline 36 & N27 8" Gage & NI & & & 72 & USW UZ-N79 & .32 & $2 / 19$ & & 106 & 4JA (8") & NI & & \\
\hline 37 & USW UZ-N35 & NI & & & 73 & USW UZ-N80 & .38 & $2 / 19$ & & 107 & Knothead Gap & NI & & \\
\hline 38 & USW UZ-N36 & NI & & & 73 & N80 4" Gage & .42 & $2 / 19$ & & 108 & Upper Forty Mile & $\mathrm{NI}$ & & \\
\hline
\end{tabular}


Table 2. Summary of precipitation data from the nonrecording gage network at Yucca Mountain, Nevada, for water years 1992 and 1993 --Continued

\begin{tabular}{|c|c|c|c|c|c|c|c|c|c|c|c|c|c|c|}
\hline $\begin{array}{l}\text { Loca- } \\
\text { tion } \\
\text { code }\end{array}$ & $\begin{array}{c}\text { Site } \\
\text { nomenclature }\end{array}$ & $\begin{array}{l}\text { Amount } \\
\text { (inches) }\end{array}$ & $\begin{array}{l}\text { Date } \\
\text { read }\end{array}$ & Notes & $\begin{array}{l}\text { Loca- } \\
\text { tion } \\
\text { code }\end{array}$ & $\begin{array}{c}\text { Site } \\
\text { nomenclature }\end{array}$ & $\begin{array}{l}\text { Amount } \\
\text { (inehes) }\end{array}$ & $\begin{array}{l}\text { Date } \\
\text { read }\end{array}$ & Notes & $\begin{array}{l}\text { Loca- } \\
\text { tion } \\
\text { code }\end{array}$ & $\begin{array}{c}\text { Site } \\
\text { nomenclature }\end{array}$ & $\begin{array}{l}\text { Amount } \\
\text { (inches) }\end{array}$ & $\begin{array}{l}\text { Date } \\
\text { read }\end{array}$ & Notes \\
\hline \multicolumn{15}{|c|}{ STORM OF MARCH 2, 1992} \\
\hline 1 & UE-25 UZN \#1 & .88 & $3 / 4$ & & 39 & USW UZ-N37 & .82 & $3 / 4$ & & 74 & USW UZ-N81 & 1.30 & $3 / 5$ & \\
\hline 2 & UE-25 UZN \#2 & .93 & $3 / 4$ & & 39 & N37 4" Gage & NI & & & 75 & USW UZ-N82 & 1.28 & $3 / 5$ & \\
\hline 3 & UE-25 UZN \#3 & .82 & $3 / 4$ & & 40 & USW UZ-N40 & .85 & $3 / 4$ & & 75 & N82 4" Gage & 1.33 & $3 / 5$ & \\
\hline 4 & UE-25 UZN \#4 & .85 & $3 / 4$ & & 40 & N40 4" Gage & NI & & & 76 & USW UZ-N83 & 1.28 & $3 / 5$ & \\
\hline 4 & N4 4" Gage & NI & & & 41 & USW UZ-N41 & .90 & $3 / 4$ & & 77 & USW UZ-N84 & 1.22 & $3 / 5$ & \\
\hline 5 & UE-25 UZN \#5 & .84 & $3 / 4$ & & 42 & USW UZ-N42 & .97 & $3 / 4$ & & 78 & USW UZ-N86 & 1.29 & $3 / 5$ & \\
\hline 6 & UE-25 UZN \#6 & .85 & $3 / 4$ & & 43 & USW UZ-N43 & .95 & $3 / 4$ & & 79 & USW UZ-N87 & 1.25 & $3 / 5$ & \\
\hline 7 & UE-25 UZN \#7 & .81 & $3 / 4$ & & 43 & N43 4" Gage & $\mathrm{NI}$ & & & 80 & USW UZ-N88 & 1.23 & $3 / 5$ & \\
\hline 8 & UE-25 UZN \#8 & .84 & $3 / 4$ & & 44 & USW UZ-N44 & .97 & $3 / 4$ & & 81 & USW UZ-N89 & 1.28 & $3 / 5$ & \\
\hline 9 & UE-25 UZN \#9 & .81 & $3 / 4$ & & 45 & USW UZ-N45 & .94 & $3 / 4$ & & 82 & USW UZ-N90 & 1.22 & $3 / 5$ & \\
\hline 10 & UE-25 UZN \#10 & .90 & $3 / 4$ & & 46 & USW UZ-N46 & 1.60 & $3 / 4$ & & 82 & N90 4" Gage & 1.34 & $3 / 5$ & \\
\hline 10 & N10 4" Gage & NI & & & 47 & USW UZ-N47 & 1.10 & $3 / 4$ & & 83 & USW UZ-N93 & 1.50 & $3 / 4$ & ** \\
\hline 11 & UE-25 UZN \#12 & .85 & $3 / 4$ & & 48 & USW UZ-N48 & 1.01 & $3 / 4$ & & 84 & USW UZ-N94 & 1.58 & $3 / 4$ & $* *$ \\
\hline 12 & UE-25 UZN \#13 & .90 & $3 / 4$ & & 49 & USW UZ-N49 & .99 & $3 / 4$ & & 85 & USW UZ-N95 & 1.49 & $3 / 4$ & $* *$ \\
\hline 13 & UE-25 UZN \#14 & .81 & $3 / 4$ & & 50 & USW UZ-N50 & .98 & $3 / 4$ & & 86 & USW UZ-N96 & 1.52 & $3 / 4$ & $* *$ \\
\hline 14 & UE-25 UZN \#18 & .78 & $3 / 4$ & & 50 & N50 4" Gage & NI & & & 87 & USW UZ-N98 & 1.00 & $3 / 4$ & \\
\hline 15 & UE-25 UZN \#19 & .91 & $3 / 4$ & & 51 & USW UZ-N51 & .96 & $3 / 4$ & & 87 & N98 4" Gage & NI & & \\
\hline 16 & UE-25 UZN $\# 20$ & .93 & $3 / 4$ & & 52 & USW UZ-N52 & .90 & $3 / 4$ & & 88 & USW UZ 13 & 1.10 & $3 / 4$ & *** \\
\hline 17 & UE-25 UZN \#21 & .89 & $3 / 4$ & & 53 & USW UZ-N53 & 1.30 & $3 / 4$ & *** & 88 & UZ-13 4" Gage & $\mathrm{NI}$ & & \\
\hline 17 & N21 4" Gage & NI & & & 54 & USW UZ-N54 & $\mathbf{M}$ & & & 89 & USW GA-1 & 1.12 & $3 / 4$ & \\
\hline 18 & UE-25 UZN \#22 & .89 & $3 / 4$ & & 54 & N54 4" Gage & NI & & & 90 & USW G-2 & $\mathbf{M}$ & & \\
\hline 19 & UE-25 UZN $\# 23$ & .92 & $3 / 4$ & & 55 & USW UZ-N55 & .38 & $3 / 4$ & & 91 & UE25 WT-4 & .76 & $3 / 4$ & \\
\hline 20 & UE-25 UZN \#28 & .91 & $3 / 4$ & & 56 & USW UZ-N57 & 1.04 & $3 / 4$ & & 92 & UE25 WT-18 & .78 & $3 / 4$ & \\
\hline 21 & UE-25 UZN \#29 & .91 & $3 / 4$ & & 57 & USW UZ-N64 & NI & & & 93 & USW H-5 & 1.03 & $3 / 4$ & \\
\hline 22 & UE-25 UZN \#30 & .95 & $3 / 4$ & & 58 & USW UZ-N65 & 1.20 & $3 / 4$ & ** & 94 & HRF Wedge & .42 & $3 / 4$ & \\
\hline
\end{tabular}




\begin{tabular}{|c|c|c|c|c|c|c|c|c|c|c|c|c|c|c|}
\hline & & IN & əI!W Krog Iadd $_{\Omega}$ & $80 I$ & & $\mathcal{S / \mathcal { E }}$ & $6 \mathrm{VI}$ & 288 „. & $\varepsilon L$ & & & IN & $9 \varepsilon N-Z \cap M S \cap$ & $8 \varepsilon$ \\
\hline & & IN & deg praypoury & LOI & & $\varsigma / \mathfrak{\varepsilon}$ & ZI'I & $08 \mathrm{~N}-\mathrm{Z} \cap \mathrm{MS} \Omega$ & $\varepsilon\llcorner$ & & & IN & SEN-Zn MSח & $L \mathcal{E}$ \\
\hline & & IN & $(. .8) \forall[t$ & 901 & & $\varsigma / \varepsilon$ & $8 Z^{*} I$ & $6 L N-\mathrm{Z} \Omega \mathrm{MS} \Omega$ & $z L$ & & & IN & 28ีED .8 LZN & $9 \varepsilon$ \\
\hline & $\$ / \mathcal{E}$ & $0 I \cdot I$ & $\| ! ! H$ s̊n!d & sol & & $\$ / \varepsilon$ & $2 \tau \cdot I$ & $8 \angle N-Z \cap M S \cap$ & $\mathrm{I} L$ & & $t / \varepsilon$ & OI'I & $\angle Z N-Z \cap M S \cap$ & $9 \varepsilon$ \\
\hline & & IN & 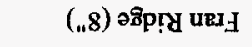 & t0I & & $\varsigma / \varepsilon$ & $6 Z^{*} \mathrm{I}$ & כ8ES „ $t L L N$ & $O L$ & & $t / \varepsilon$ & $10^{\circ} \mathrm{I}$ & $9 Z N-Z \cap M S \cap$ & $\varsigma \varepsilon$ \\
\hline \multirow[t]{7}{*}{$* *$} & $\downarrow / \varepsilon$ & $\$ 8^{\circ}$ & 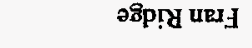 & toI & & $\varsigma / \mathfrak{\varepsilon}$ & $Z Z \cdot I$ & $\angle L N^{-} \mathrm{Z} \cap M S \cap$ & $0 L$ & & $t / \mathcal{E}$ & to. I & $S Z N-Z \cap M S \cap$ & $\downarrow \varepsilon$ \\
\hline & & IN & Uọ̣ts EәM & £oI & & $\varsigma / \mathcal{E}$ & $60^{\circ} \mathrm{I}$ & $9 \angle \mathrm{N}-\mathrm{Z} \cap \mathrm{MS} \cap$ & 69 & & & IN & 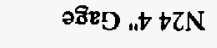 & $\varepsilon \varepsilon$ \\
\hline & $t / \varepsilon$ & 101 & Uop̣p1s RəM & EOI & & & IN & 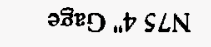 & 89 & & $t / \varepsilon$ & $90^{\circ} \mathrm{I}$ & $t Z N-Z \cap M S \cap$ & $\varepsilon \varepsilon$ \\
\hline & & IN & uọne1s Eə⿳亠丷厂 & 201 & & $\$ / \varepsilon$ & $80^{\circ} \mathrm{I}$ & $S \angle N-Z \cap M S \cap$ & 89 & & & IN & $\angle I N-Z \cap M S \cap$ & $\tau \varepsilon$ \\
\hline & & IN & UO!̣EIS E्M & I0I & & $\mathfrak{s / \mathfrak { E }}$ & $20^{\circ} \mathrm{I}$ & $t \angle N-Z \cap M S \cap$ & $\angle 9$ & & & IN & $9 I N-Z \cap M S \cap$ & IE \\
\hline & $\nabla / \mathcal{E}$ & $\mathcal{E} L^{\circ}$ & Uo!̣ets ЕәM & 101 & & $\varsigma / \mathcal{\varepsilon}$ & $0 I^{\prime} I$ & $\varepsilon L N-Z \cap M S \cap$ & 99 & & & IN & SIN-Zn MS $\Omega$ & $0 \mathcal{E}$ \\
\hline & & IN & คสิษ . . $8 \mathcal{E} \varepsilon$ & 001 & & $\mathcal{S} / \mathcal{E}$ & $66^{\circ}$ & $Z L N^{-} Z \cap M S \cap$ & \$9 & & & IN & 28ిש0 „ t IIN & 62 \\
\hline \multirow[t]{2}{*}{$* *$} & $t / \varepsilon$ & SI'I & $E-D$ & $00 I$ & & & IN & $28 \mathrm{eg} \ldots I L N$ & t9 & & & IN & IIN-Zn MSA & 62 \\
\hline & & IN & uọ̣ms peM & 66 & & $S / \mathcal{E}$ & $\varepsilon 0^{\circ} \mathrm{I}$ & $\mathrm{I} \angle \mathrm{N}-\mathrm{Z} \cap \mathrm{MS} \cap$ & $t 9$ & & & IN & $2880 . .616 \mathrm{~N}$ & 82 \\
\hline \multirow[t]{4}{*}{$* *$} & $t / \mathfrak{\varepsilon}$ & It'I & uop̣p?s $\varpi_{M}$ & 66 & & & IN & əริeด „, 0LN & घ9 & & $t / \mathcal{\varepsilon}$ & $t 9^{\circ}$ & $16 \# \mathrm{NZ} \cap 62-\mathrm{a} \cap$ & $8 z$ \\
\hline & $t / \varepsilon$ & $L \varepsilon^{*}$ & บo!nejodeAg & 86 & & $t / \varepsilon$ & $86^{\circ}$ & $0 L N-Z \cap M S \cap$ & $\varepsilon 9$ & & $\mathfrak{t} / \mathfrak{\varepsilon}$ & $16^{\circ}$ & 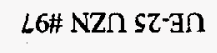 & $L 2$ \\
\hline & $\varsigma / \varepsilon$ & $z 2 \cdot 1$ & uọ̣?s вәм & $\angle 6$ & $* *$ & $t / \varepsilon$ & $Z I \cdot I$ & $69 \mathrm{~N}-\mathrm{Z} \cap \mathrm{MS} \Omega$ & $z 9$ & & & IN & 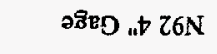 & $9 z$ \\
\hline & $\nabla / \mathcal{E}$ & $\$ 6$ & uop̣ts вอM & 96 & $* *$ & $t / \varepsilon$ & $8 \mathrm{I}^{\prime} \mathrm{I}$ & $89 \mathrm{~N}-\mathrm{Z} \cap \mathrm{MS} \cap$ & [9 & & $t / \mathfrak{E}$ & $\varepsilon L^{\prime}$ & 乙6\# NZn $\varsigma Z-\exists \Omega$ & 92 \\
\hline \multirow[t]{4}{*}{ ** } & $\downarrow / \mathcal{E}$ & $0 I^{\circ} \mathrm{I}$ & $\varepsilon-H M S \cap$ & $\$ 6$ & & & IN & $28 \mathrm{eO}$ แ $\angle 9 \mathrm{~N}$ & 09 & & $t / \mathfrak{\varepsilon}$ & $\varepsilon 9^{\circ}$ & $\varsigma 8 \# N Z \cap \varsigma Z-\exists \cap$ & $£ \mathcal{Z}$ \\
\hline & $t / \varepsilon$ & $t t^{\circ}$ & 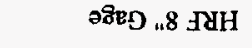 & t6 & $* *$ & $t / \varepsilon$ & $\angle I^{\prime} I$ & $\angle 9 N^{-} \mathrm{Z} \cap M S \cap$ & 09 & & $t / \mathfrak{\varepsilon}$ & $08^{\circ}$ & 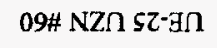 & $t z$ \\
\hline & $t / \mathfrak{E}$ & $t t^{\circ}$ & 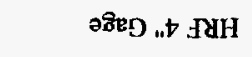 & $\$ 6$ & & $t / \mathcal{\varepsilon}$ & $00^{\circ} \mathrm{I}$ & $99 \mathrm{~N}^{-Z} \mathrm{Z} \cap \mathrm{MS} \Omega$ & $6 \subseteq$ & & $t / \varepsilon$ & $06^{\circ}$ & $9 \varsigma \#$ NZח $\varsigma Z-\exists \Omega$ & $\varepsilon 乙$ \\
\hline & & & & & ponup & $20-266$ & 'Z HО४УV & I HO WXOLS & & & & & & \\
\hline saloN & $\begin{array}{l}\text { pees } \\
\text { elea }\end{array}$ & $\begin{array}{l}\text { (seysul) } \\
\text { Junowy }\end{array}$ & 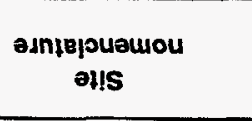 & $\begin{array}{c}\text { өpos } \\
\text { Ho! } \\
\text { - E007 }\end{array}$ & SelON & $\begin{array}{l}\text { pees } \\
\text { oled }\end{array}$ & $\begin{array}{l}\text { (seyou!) } \\
\text { funowy }\end{array}$ & $\begin{array}{c}\text { o.niejouewou } \\
\text { els }\end{array}$ & $\begin{array}{c}\text { opos } \\
\text { uop } \\
- \text {-e007 }\end{array}$ & SEION & $\begin{array}{l}\text { peed } \\
\text { eled }\end{array}$ & $\begin{array}{l}\text { (soupu!) } \\
\text { funowy }\end{array}$ & $\begin{array}{c}\text { exnepoueuou } \\
\text { ells }\end{array}$ & $\begin{array}{l}\text { opos } \\
\text { uoll } \\
\text {-E007 }\end{array}$ \\
\hline
\end{tabular}


Table 2. Summary of precipitation data from the nonrecording gage network at Yucca Mountain, Nevada, for water years 1992 and 1993 --Continued

\begin{tabular}{|c|c|c|c|c|c|c|c|c|c|c|c|c|c|c|}
\hline $\begin{array}{l}\text { Loca- } \\
\text { tion } \\
\text { code }\end{array}$ & $\begin{array}{c}\text { Site } \\
\text { nomenclature }\end{array}$ & $\begin{array}{l}\text { Amount } \\
\text { (inches) }\end{array}$ & $\begin{array}{l}\text { Date } \\
\text { read }\end{array}$ & Notes & $\begin{array}{l}\text { Loca- } \\
\text { tion } \\
\text { code }\end{array}$ & $\begin{array}{c}\text { Site } \\
\text { nomenclature }\end{array}$ & $\begin{array}{l}\text { Amount } \\
\text { (inches) }\end{array}$ & $\begin{array}{l}\text { Date } \\
\text { read }\end{array}$ & Notes & $\begin{array}{l}\text { Loca- } \\
\text { tion } \\
\text { code }\end{array}$ & $\begin{array}{c}\text { Site } \\
\text { nomenclature }\end{array}$ & $\begin{array}{l}\text { Amount } \\
\text { (inches) }\end{array}$ & $\begin{array}{l}\text { Date } \\
\text { read }\end{array}$ & Notes \\
\hline \multicolumn{15}{|c|}{ STORM OF MARCH 7-9, 1992} \\
\hline 1 & UE-25 UZN \#1 & .20 & $3 / 10$ & & 39 & USW UZ-N37 & .21 & $3 / 10$ & & 74 & USW UZ-N81 & .20 & 3/9 & \\
\hline 2 & UE-25 UZN \#2 & .29 & $3 / 10$ & & 39 & N37 4" Gage & NI & & & 75 & USW UZ-N82 & .15 & 3/9 & \\
\hline 3 & UE-25 UZN \#3 & 16 & $3 / 10$ & & 40 & USW UZ-N40 & .15 & $3 / 10$ & & 75 & N82 4" Gage & .31 & 3/9 & \\
\hline 4 & UE-25 UZN \#4 & .18 & $3 / 10$ & & 40 & N40 4" Gage & NI & & & 76 & USW UZ-N83 & .22 & $3 / 9$ & \\
\hline 4 & N4 4" Gage & NI & & & 41 & USW UZ-N41 & .18 & $3 / 10$ & & 77 & USW UZ-N84 & .20 & $3 / 9$ & \\
\hline 5 & UE-25 UZN \#5 & .16 & $3 / 10$ & & 42 & USW UZ-N42 & .17 & $3 / 10$ & & 78 & USW UZ-N86 & .22 & $3 / 9$ & \\
\hline 6 & UE-25 UZN \#6 & .17 & $3 / 10$ & & 43 & USW UZ-N43 & .18 & $3 / 10$ & & 79 & USW UZ-N87 & .20 & $3 / 9$ & \\
\hline 7 & UE-25 UZN \#7 & .21 & $3 / 10$ & & 43 & N43 4" Gage & NI & & & 80 & USW UZ-N88 & .17 & 3/9 & \\
\hline 8 & UE-25 UZN \#8 & .19 & $3 / 10$ & & 44 & USW UZ-N44 & .19 & $3 / 10$ & & 81 & USW UZ-N89 & .14 & $3 / 9$ & \\
\hline 9 & UE-25 UZN \#9 & .21 & $3 / 10$ & & 45 & USW UZ-N45 & .19 & $3 / 10$ & & 82 & USW UZ-N90 & .20 & $3 / 9$ & \\
\hline 10 & UE-25 UZN \#10 & .20 & $3 / 10$ & & 46 & USW UZ-N46 & .25 & $3 / 10$ & & 82 & N90 4" Gage & .34 & $3 / 9$ & \\
\hline 10 & N10 4" Gage & NI & & & 47 & USW UZ-N47 & .15 & $3 / 10$ & & 83 & USW UZ-N93 & .23 & $3 / 11$ & \\
\hline 11 & UE-25 UZN \#12 & .18 & $3 / 10$ & & 48 & USW UZ-N48 & .19 & $3 / 11$ & & 84 & USW UZ-N94 & .19 & $3 / 11$ & \\
\hline 12 & UE-25 UZN \#13 & .28 & $3 / 10$ & & 49 & USW UZ-N49 & .20 & $3 / 11$ & & 85 & USW UZ-N95 & .21 & $3 / 11$ & \\
\hline 13 & UE-25 UZN \#14 & .17 & $3 / 10$ & & 50 & USW UZ-N50 & .18 & $3 / 11$ & & 86 & USW UZ-N96 & .18 & $3 / 11$ & \\
\hline 14 & UE-25 UZN \#18 & .15 & $3 / 10$ & & 50 & N50 4" Gage & NI & & & 87 & USW UZ-N98 & .14 & $3 / 10$ & \\
\hline 15 & UE-25 UZN \#19 & .22 & $3 / 11$ & & 51 & USW UZ-N51 & .19 & $3 / 11$ & & 87 & N98 4" Gage & NI & & \\
\hline 16 & UE-25 UZN \#20 & .20 & $3 / 11$ & & 52 & USW UZ-N52 & .19 & $3 / 11$ & & 88 & USW UZ 13 & .18 & $3 / 11$ & \\
\hline 17 & UE-25 UZN \#21 & .21 & $3 / 11$ & & 53 & USW UZ-N53 & .20 & $3 / 11$ & & 88 & UZ-13 4" Gage & NI & & \\
\hline 17 & N21 4" Gage & NI & & & 54 & USW UZ-N54 & $\mathbf{M}$ & & & 89 & USW GA-1 & .23 & $3 / 10$ & \\
\hline 18 & UE-25 UZN \#22 & .21 & $3 / 11$ & & 54 & N54 4" Gage & NI & & & 90 & USW G-2 & .23 & $3 / 10$ & \\
\hline 19 & UE-25 UZN \#23 & .22 & $3 / 11$ & & 55 & USW UZ-N55 & .18 & $3 / 11$ & & 91 & UE25 WT-4 & .14 & $3 / 10$ & \\
\hline 20 & UE-25 UZN \#28 & .19 & $3 / 11$ & & 56 & USW UZ-N57 & .16 & $3 / 11$ & & 92 & UE25 WT-18 & .26 & $3 / 10$ & \\
\hline 21 & UE-25. UZN \#29 & .21 & $3 / 11$ & & 57 & USW UZ-N64 & NI & & & 93 & USW H-5 & .15 & $3 / 10$ & \\
\hline 22 & UE-25 UZN \#30 & .22 & $3 / 11$ & & 58 & USW UZ-N65 & .14 & $3 / 12$ & & 94 & HRF Wedge & .62 & $3 / 10$ & \\
\hline
\end{tabular}


Table 2. Summary of precipitation data from the nonrecording gage network at Yucca Mountain, Nevada, for water years 1992 and $1993--$ Continued

\begin{tabular}{|c|c|c|c|c|c|c|c|c|c|c|c|c|c|c|}
\hline $\begin{array}{l}\text { Loca- } \\
\text { tion } \\
\text { code }\end{array}$ & $\begin{array}{c}\text { Site } \\
\text { nomenclature }\end{array}$ & $\begin{array}{l}\text { Amount } \\
\text { (inches) }\end{array}$ & $\begin{array}{l}\text { Date } \\
\text { read }\end{array}$ & Notes & $\begin{array}{l}\text { Loca- } \\
\text { tion } \\
\text { code }\end{array}$ & $\begin{array}{c}\text { Site } \\
\text { nomenclature }\end{array}$ & $\begin{array}{l}\text { Amount } \\
\text { (inches) }\end{array}$ & $\begin{array}{l}\text { Date } \\
\text { read }\end{array}$ & Notes & $\begin{array}{l}\text { Loca- } \\
\text { tion } \\
\text { code }\end{array}$ & $\begin{array}{c}\text { Site } \\
\text { nomenclature }\end{array}$ & $\begin{array}{l}\text { Amount } \\
\text { (inches) }\end{array}$ & $\begin{array}{l}\text { Date } \\
\text { read }\end{array}$ & Notes \\
\hline \multicolumn{15}{|c|}{ STORM OF MARCH 7-9, 1992-Contlnued } \\
\hline 23 & UE-25 UZN \#56 & .19 & $3 / 11$ & & 59 & USW UZ-N66 & .19 & $3 / 12$ & & 94 & HRF 4" Gage & .64 & $3 / 10$ & \\
\hline 24 & UE-25 UZN \#60 & .15 & $3 / 11$ & & 60 & USW UZ-NG7 & .16 & $3 / 11$ & & 94 & HRF $\overline{8}^{\prime \prime}$ Gage & .65 & $3 / 10$ & \\
\hline 25 & UE-25 UZN \#85 & .36 & $3 / 11$ & & 60 & N67 4" Gage & NI & & & 95 & USW H-3 & .18 & $3 / 11$ & \\
\hline 26 & UE-25 UZN \#92 & .17 & $3 / 9$ & & 61 & USW UZ-N68 & .16 & $3 / 12$ & & 96 & Wea station & .20 & $3 / 11$ & \\
\hline 26 & N92 4" Gage & $\mathrm{NI}$ & & & 62 & USW UZ-N69 & .16 & $3 / 12$ & & 97 & Wea station & $\mathbf{M}^{*}$ & & \\
\hline 27 & UE-25 UZN \#97 & .19 & $3 / 11$ & & 63 & USW UZ-N70 & .14 & $3 / 10$ & & 98 & Evaporation & .53 & $3 / 11$ & \\
\hline 28 & UE-29 UZN \#91 & .18 & $3 / 9$ & & 63 & N70 4" Gage & NI & & & 99 & Wea station & .20 & $3 / 11$ & \\
\hline 28 & N91 4" Gage & $\mathrm{NI}$ & & & 64 & USW UZ-N71 & .17 & $3 / 11$ & & 99 & Wea station & NI & & \\
\hline 29 & USW UZ-N11 & NI & & & 64 & N71 4" Gage & $\mathrm{NI}$ & & & 100 & G-3 & .22 & $3 / 11$ & \\
\hline 29 & N11 4" Gage & $\mathrm{NI}$ & & & 65 & USW UZ-N72 & .16 & $3 / 11$ & & 100 & G-3 8" Gage & NI & & \\
\hline 30 & USW UZ-N15 & NI & & & 66 & USW UZ-N73 & .22 & $3 / 11$ & & 101 & Wea station & .17 & $3 / 11$ & \\
\hline 31 & USW UZ-N16 & NI & & & 67 & USW UZ-N74 & .18 & $3 / 11$ & & 101 & Wea station & $\mathrm{NI}$ & & \\
\hline 32 & USW UZ-N17 & NI & & & 68 & USW UZ-N75 & .17 & $3 / 11$ & & 102 & Wea station & NI & & \\
\hline 33 & USW UZ-N24 & .15 & $3 / 10$ & & 68 & N75 4" Gage & NI & & & 103 & Wea station & .15 & $3 / 10$ & \\
\hline 33 & N24 4" Gage & NI & & & 69 & USW UZ-N76 & .16 & $3 / 11$ & & 103 & Wea station & NI & & \\
\hline 34 & USW UZ-N25 & .15 & $3 / 10$ & & 70 & USW UZ-N77 & .16 & $3 / 9$ & & 104 & Fran Ridge & .19 & $3 / 19$ & \\
\hline 35 & USW UZ-N26 & .15 & $3 / 10$ & & 70 & N77 4" Gage & $\mathbf{M}$ & $3 / 9$ & & 104 & Fran Ridge (8") & NI & & \\
\hline 36 & USW UZ-N27 & .15 & $3 / 11$ & & 71 & USW UZ-N78 & .20 & $3 / 9$ & & 105 & Plug Hill & .15 & $3 / 9$ & \\
\hline 36 & N27 8" Gage & NI & & & 72 & USW UZ-N79 & .24 & $3 / 9$ & & 106 & 4JA (8") & NI & & \\
\hline 37 & USW UZ-N35 & NI & & & 73 & USW UZ-N80 & .25 & $3 / 9$ & & 107 & Knothead Gap & NI & & \\
\hline 38 & USW UZ-N36 & $\mathrm{NI}$ & & & 73 & N80 4" Gage & .40 & $3 / 9$ & & 108 & Upper Forty Mile & NI & & \\
\hline
\end{tabular}


Table 2. Summary of precipitation data from the nonrecording gage network at Yucca Mountain, Nevada, for water years 1992 and $1993--$ Continued

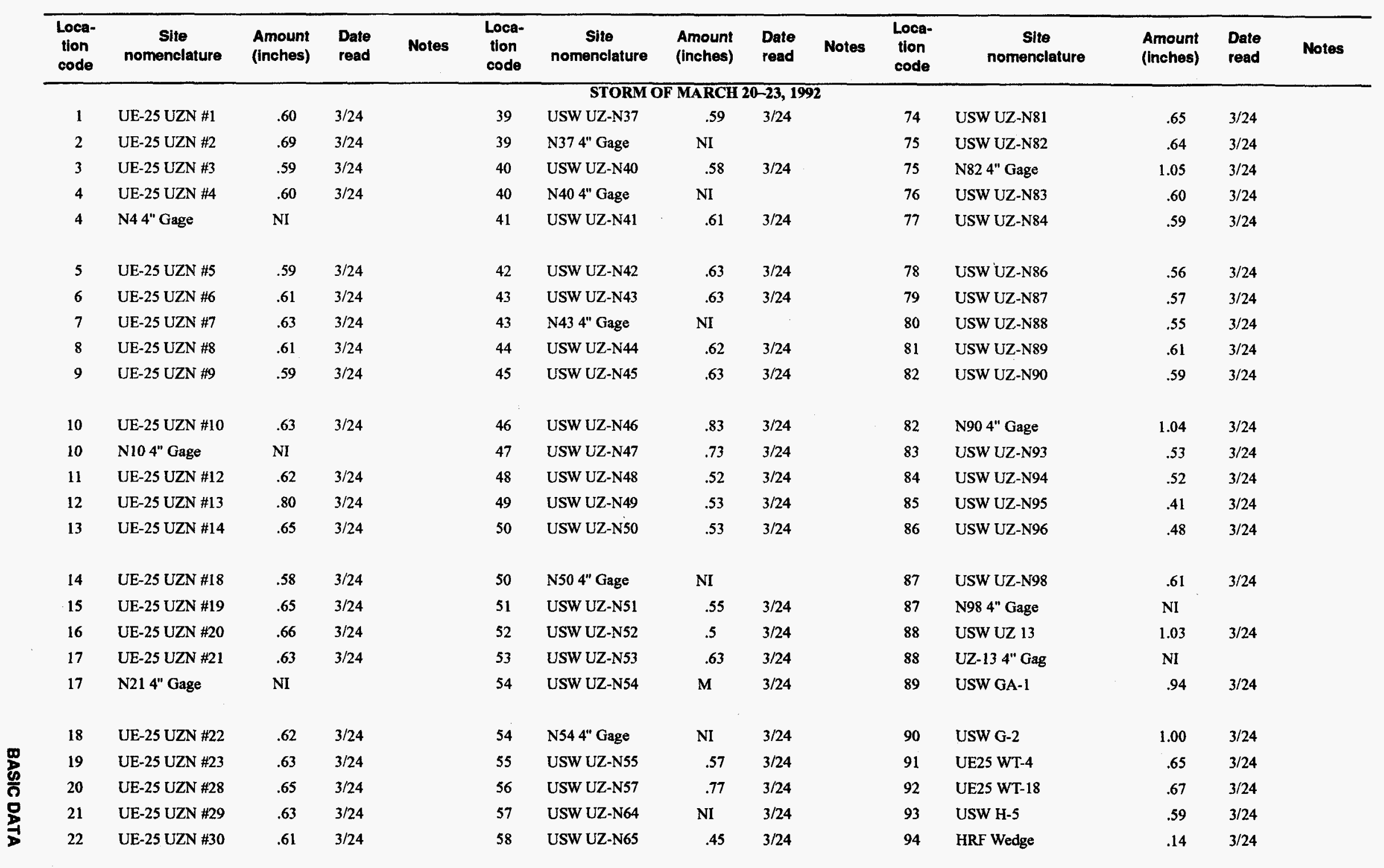




\begin{tabular}{|c|c|c|c|c|c|c|c|c|c|c|c|c|c|c|}
\hline $\begin{array}{l}\text { Loca- } \\
\text { tlon } \\
\text { code }\end{array}$ & $\begin{array}{c}\text { Site } \\
\text { nomenclature }\end{array}$ & $\begin{array}{l}\text { Amount } \\
\text { (inches) }\end{array}$ & $\begin{array}{l}\text { Date } \\
\text { read }\end{array}$ & Notes & $\begin{array}{l}\text { Loca- } \\
\text { tion } \\
\text { code }\end{array}$ & $\begin{array}{c}\text { Site } \\
\text { nomenclature }\end{array}$ & $\begin{array}{l}\text { Amount } \\
\text { (inches) }\end{array}$ & $\begin{array}{l}\text { Date } \\
\text { read }\end{array}$ & Notes & $\begin{array}{l}\text { Loca- } \\
\text { tion } \\
\text { code }\end{array}$ & $\begin{array}{c}\text { Site } \\
\text { nomenclature }\end{array}$ & $\begin{array}{l}\text { Amount } \\
\text { (inches) }\end{array}$ & $\begin{array}{l}\text { Date } \\
\text { read }\end{array}$ & Notes \\
\hline \multicolumn{15}{|c|}{ STORM OF MARCH 20-23, 1992-Continued } \\
\hline 23 & UE-25 UZN \#56 & .59 & $3 / 24$ & & 59 & USW UZ-N66 & .52 & $3 / 24$ & & 94 & HRF 4" Gage & .23 & $3 / 24$ & \\
\hline 24 & UE-25 UZN \#60 & .64 & $3 / 24$ & & 60 & USW UZ-N67 & .84 & $3 / 24$ & & 94 & HRF 8" Gage & .23 & $3 / 24$ & \\
\hline 25 & UE-25 UZN \#85 & .71 & $3 / 24$ & & 60 & N67 4" Gage & NI & $3 / 24$ & & 95 & USW H-3 & .79 & $3 / 24$ & \\
\hline 26 & UE-25 UZN $\# 92$ & .95 & $3 / 23$ & & 61 & USW UZ-N68 & .88 & $3 / 24$ & & 96 & Wea station & .53 & $3 / 24$ & \\
\hline 26 & N92 4" Gage & NI & & & 62 & USW UZ-N69 & .90 & $3 / 24$ & & 97 & Wea station & $\mathbf{M}^{*}$ & & \\
\hline 27 & UE-25 UZN \#97 & .60 & $3 / 24$ & & 63 & USW UZ-N70 & .61 & $3 / 24$ & & 98 & Evaporation & .22 & $3 / 24$ & \\
\hline 28 & UE-29 UZN \#91 & 1.08 & $3 / 23$ & & 63 & N70 4" Gage & NI & & & 99 & Wea station & .80 & $3 / 24$ & \\
\hline 28 & N91 4" Gage & NI & & & 64 & USW UZ-N71 & .55 & $3 / 24$ & & 99 & Wea station & NI & & \\
\hline 29 & USW UZ-N11 & NI & & & 64 & N71 4" Gage & NI & & & 100 & G-3 & 1.13 & $3 / 24$ & \\
\hline 29 & N11 4" Gage & NI & & & 65 & USW UZ-N72 & .56 & $3 / 24$ & & 100 & G-3 8" Gage & $\mathrm{NI}$ & & \\
\hline 30 & USW UZ-N15 & NI & & & 66 & USW UZ-N73 & .57 & $3 / 24$ & & 101 & Wea station & .75 & $3 / 24$ & \\
\hline 31 & USW UZ-N16 & NI & & & 67 & USW UZ-N74 & .57 & $3 / 24$ & & 101 & Wea station & NI & & \\
\hline 32 & USW UZ-N17 & NI & & & 68 & USW UZ-N75 & .57 & $3 / 24$ & & 102 & Wea station & NI & & \\
\hline 33 & USW UZ-N24 & .66 & $3 / 24$ & & 68 & N75 4" Gage & NI & & & 103 & Wea station & .65 & $3 / 24$ & \\
\hline 33 & N24 4" Gage & NI & & & 69 & USW UZ-N76 & .55 & $3 / 24$ & & 103 & Wea station & NI & & \\
\hline 34 & USW UZ-N25 & .64 & $3 / 24$ & & 70 & USW UZ-N77 & .67 & $3 / 24$ & EVAP & 104 & Fran Ridge & .45 & $3 / 24$ & \\
\hline 35 & USW UZ-N26 & .63 & $3 / 24$ & & 70 & N77 4" Gage & .62 & $3 / 24$ & & 104 & Fran Ridge (8") & NI & & \\
\hline 36 & USW UZ-N27 & .71 & $3 / 24$ & & 71 & USW UZ-N78 & .56 & $3 / 24$ & & 105 & Plug Hill & .94 & $3 / 24$ & \\
\hline 36 & N27 8" Gage & NI & & & 72 & USW UZ-N79 & .63 & $3 / 24$ & & 106 & 4JA (8") & NI & & \\
\hline 37 & USW UZ-N35 & NI & & & 73 & USW UZ-N80 & .63 & $3 / 24$ & & 107 & Knothead Gap & NI & & \\
\hline 38 & USW UZ-N36 & NI & & & 73 & N80 4" Gage & .63 & $3 / 24$ & & 108 & Upper Forty Mile & NI & & \\
\hline
\end{tabular}


Table 2. Summary of precipitation data from the nonrecording gage network at Yucca Mountain, Nevada, for water years 1992 and 1993 --Continued

\begin{tabular}{|c|c|c|c|c|c|c|c|c|c|c|c|c|c|c|}
\hline $\begin{array}{l}\text { Loca- } \\
\text { tion } \\
\text { code }\end{array}$ & $\begin{array}{c}\text { Site } \\
\text { nomenclature }\end{array}$ & $\begin{array}{l}\text { Amount } \\
\text { (inches) }\end{array}$ & $\begin{array}{l}\text { Date } \\
\text { read }\end{array}$ & Notes & $\begin{array}{l}\text { Loca- } \\
\text { tion } \\
\text { code }\end{array}$ & $\begin{array}{c}\text { Site } \\
\text { nomenclature }\end{array}$ & $\begin{array}{l}\text { Amount } \\
\text { (inches) }\end{array}$ & $\begin{array}{l}\text { Date } \\
\text { read }\end{array}$ & Notes & $\begin{array}{l}\text { Loca- } \\
\text { tion } \\
\text { code }\end{array}$ & $\begin{array}{c}\text { Site } \\
\text { nomenclature }\end{array}$ & $\begin{array}{l}\text { Amount } \\
\text { (inches) }\end{array}$ & $\begin{array}{l}\text { Date } \\
\text { read }\end{array}$ & Notes \\
\hline \multicolumn{15}{|c|}{ STORM OF MARCH 27, 1992} \\
\hline 1 & UE-25 UZN \#1 & .25 & $3 / 30$ & & 39 & USW UZ-N37 & .22 & $3 / 30$ & & 74 & USW UZ-N81 & $\mathbf{M}^{*}$ & & \\
\hline 2 & UE-25 UZN \#2 & .36 & $3 / 30$ & & 39 & N37 4" Gage & NI & & & 75 & USW UZ-N82 & $\mathbf{M}^{*}$ & & \\
\hline 3 & UE-25 UZN \#3 & .28 & $3 / 30$ & & 40 & USW UZ-N40 & .22 & $3 / 30$ & & 75 & N82 4" Gage & $\mathbf{M}^{*}$ & & \\
\hline 4 & UE-25 UZN \#4 & .29 & $3 / 30$ & & 40 & N40 4" Gage & NI & & & 76 & USW UZ-N83 & $\mathbf{M}^{*}$ & & \\
\hline 4 & N4 4" Gage & NI & & & 41 & USW UZ-N41 & .21 & $3 / 30$ & & 77 & USW UZ-N84 & $\mathrm{M}^{*}$ & & \\
\hline 5 & UE-25 UZN \#5 & .26 & $3 / 30$ & & 42 & USW UZ-N42 & .17 & $3 / 30$ & & 78 & USW UZ-N86 & $\mathbf{M}^{*}$ & & \\
\hline 6 & UE-25 UZN \#6 & .28 & $3 / 30$ & & 43 & USW UZ-N43 & .21 & $3 / 30$ & & 79 & USW UZ-N87 & $\mathbf{M}^{*}$ & & \\
\hline 7 & UE-25 UZN \#7 & .28 & $3 / 30$ & & 43 & N43 4" Gage & NI & & & 80 & USW UZ-N88 & $\mathbf{M}^{*}$ & & \\
\hline 8 & UE-25 UZN \#8 & .28 & $3 / 30$ & & 44 & USW UZ-N44 & .22 & $3 / 30$ & & 81 & USW UZ-N89 & $\mathbf{M}^{*}$ & & \\
\hline 9 & UE-25 UZN \#9 & .27 & $3 / 30$ & & 45 & USW UZ-N45 & .20 & $3 / 30$ & & 82 & USW UZ-N90 & $\mathrm{M}^{*}$ & & \\
\hline 10 & UE-25 UZN $\# 10$ & .26 & $3 / 30$ & & 46 & USW UZ-N46 & 45 & $3 / 30$ & & 82 & N90 4" Gage & $\mathrm{M}^{*}$ & & \\
\hline 10 & N10 4" Gage & NI & & & 47 & USW UZ-N47 & .39 & $3 / 30$ & & 83 & USW UZ-N93 & .25 & $3 / 30$ & \\
\hline 11 & UE-25 UZN \#12 & .26 & $3 / 30$ & & 48 & USW UZ-N48 & $\mathbf{M}^{*}$ & & & 84 & USW UZ-N94 & .29 & $3 / 30$ & \\
\hline 12 & UE-25 UZN \#13 & .37 & $3 / 30$ & & 49 & USW UZ-N49 & $\mathbf{M}^{*}$ & & & 85 & USW UZ-N95 & .37 & $3 / 30$ & \\
\hline 13 & UE-25 UZN \#14 & .22 & $3 / 30$ & & 50 & USW UZ-N50 & $\mathbf{M}^{*}$ & & & 86 & USW UZ-N96 & .28 & $3 / 30$ & \\
\hline 14 & UE-25 UZN \#18 & .24 & $3 / 30$ & & 50 & N50 4" Gage & NI & & & 87 & USW UZ-N98 & .26 & $3 / 30$ & \\
\hline 15 & UE-25 UZN $\# 19$ & .24 & $3 / 30$ & & 51 & USW UZ-N51 & $\mathrm{M}^{*}$ & & & 87 & N98 4" Gage & NI & & \\
\hline 16 & UE-25 UZN $\# 20$ & .24 & $3 / 30$ & & 52 & USW UZ-N52 & $\mathbf{M}^{*}$ & & & 88 & USW UZ 13 & .23 & $3 / 30$ & \\
\hline 17 & UE-25 UZN $\# 21$ & .24 & $3 / 30$ & & 53 & USW UZ-N53 & $\mathrm{M}^{*}$ & & & 88 & UZ-13 4" Gage & NI & & \\
\hline 17 & N21 4" Gage & $\mathrm{NI}$ & & & 54 & USW UZ-N54 & $\mathbf{M}$ & & & 89 & USW GA-1 & .56 & $3 / 30$ & \\
\hline 18 & UE-25 UZN \#22 & .24 & $3 / 30$ & & 54 & N54 4" Gage & NI & & & 90 & USW G-2 & .55 & $3 / 30$ & \\
\hline 19 & UE-25 UZN $\# 23$ & .26 & $3 / 30$ & & 55 & USW UZ-N55 & $\mathbf{M}^{*}$ & & & 91 & UE25 WT-4 & .23 & $3 / 30$ & \\
\hline 20 & UE-25 UZN \#28 & .35 & $3 / 30$ & & 56 & USW UZ-N57 & .17 & $3 / 30$ & & 92 & UE25 WT-18 & .29 & $3 / 30$ & \\
\hline 21 & UE- 25 UZN $\# 29$ & .29 & $3 / 30$ & & 57 & USW UZ-N64 & $\mathrm{NI}$ & & & 93 & USW H-5 & .29 & $3 / 30$ & \\
\hline 22 & UE-25 UZN \#30 & .27 & $3 / 30$ & & 58 & USW UZ-N65 & .19 & $3 / 30$ & & 94 & HRF Wedge & .23 & $3 / 30$ & \\
\hline
\end{tabular}


o Table 2. Summary of precipitation data from the nonrecording gage network at Yucca Mountain, Nevada, for water years 1992 and 1993--Continued

\begin{tabular}{|c|c|c|c|c|c|c|c|c|c|c|c|c|c|c|}
\hline $\begin{array}{l}\text { Loca- } \\
\text { tion } \\
\text { code }\end{array}$ & $\begin{array}{c}\text { Site } \\
\text { nomenclature }\end{array}$ & $\begin{array}{l}\text { Amount } \\
\text { (inches) }\end{array}$ & $\begin{array}{l}\text { Date } \\
\text { read }\end{array}$ & Notes & $\begin{array}{l}\text { Loca- } \\
\text { tion } \\
\text { code }\end{array}$ & $\begin{array}{c}\text { Site } \\
\text { nomenelature }\end{array}$ & $\begin{array}{l}\text { Amount } \\
\text { (inches) }\end{array}$ & $\begin{array}{l}\text { Date } \\
\text { read }\end{array}$ & Notes & $\begin{array}{l}\text { Loca- } \\
\text { tion } \\
\text { code }\end{array}$ & $\begin{array}{c}\text { Site } \\
\text { nomenclature }\end{array}$ & $\begin{array}{l}\text { Amount } \\
\text { (Inches) }\end{array}$ & $\begin{array}{l}\text { Date } \\
\text { read }\end{array}$ & Notes \\
\hline \multicolumn{15}{|c|}{ STORM OF MARCH 27, 1992-Continued } \\
\hline 23 & UE-25 UZN \#56 & $\mathrm{M}^{*}$ & & & 59 & USW UZ-N66 & .20 & $3 / 30$ & & 94 & HRF 4" Gage & .28 & $3 / 30$ & \\
\hline 24 & UE-25 UZN \#60 & $\mathrm{M}^{*}$ & & & 60 & USW UZ-N67 & .23 & $3 / 30$ & & 94 & HRF 8" Gage & .28 & $3 / 30$ & \\
\hline 25 & UE-25 UZN \#85 & .14 & $3 / 30$ & & 60 & N67 4" Gage & NI & & & 95 & USW H-3 & .16 & $3 / 30$ & \\
\hline 26 & UE-25 UZN \#92 & .05 & $3 / 30$ & & 61 & USW UZ-N68 & .21 & $3 / 30$ & & 96 & Wea station & .27 & $3 / 30$ & \\
\hline 26 & N92 4" Gage & NI & & & 62 & USW UZ-N69 & .23 & $3 / 30$ & & 97 & Wea station & $\mathrm{M}^{*}$ & & \\
\hline 27 & UE-25 UZN \#97 & $\mathrm{M}^{*}$ & & & 63 & USW UZ-N70 & .36 & $3 / 30$ & & 98 & Evaporation & $\mathrm{M}^{*}$ & & \\
\hline 28 & UE-29 UZN \#91 & .70 & $3 / 30$ & & 63 & N70 4" Gage & NI & & & 99 & Wea station & .19 & $3 / 30$ & \\
\hline 28 & N91 4" Gage & NI & & & 64 & USW UZ-N71 & .23 & $3 / 30$ & & 99 & Wea station & NI & & \\
\hline 29 & USW UZ-N11 & NI & & & 64 & N71 4" Gage & $\mathrm{NI}$ & & & 100 & G-3 & .27 & $3 / 30$ & \\
\hline 29 & N11 4" Gage & NI & & & 65 & USW UZ-N72 & .21 & $3 / 30$ & & 100 & G-3 8" Gage & NI & & \\
\hline 30 & USW UZ-N15 & NI & & & 66 & USW UZ-N73 & .23 & $3 / 30$ & & 101 & Wea station & .23 & & \\
\hline 31 & USW UZ-N16 & NI & & & 67 & USW UZ-N74 & .22 & $3 / 30$ & & 101 & Wea station & NI & & \\
\hline 32 & USW UZ-N17 & $\mathrm{NI}$ & & & 68 & USW UZ-N75 & .21 & $3 / 30$ & & 102 & Wea station & NI & & \\
\hline 33 & USW UZ-N24 & .29 & $3 / 30$ & & 68 & N75 4" Gage & $\mathrm{NI}$ & & & 103 & Wea station & .35 & $3 / 30$ & \\
\hline 33 & N24 4" Gage & NI & & & 69 & USW UZ-N76 & .20 & $3 / 30$ & & 103 & Wea station & NI & & \\
\hline 34 & USW UZ-N25 & NI & $3 / 30$ & & 70 & USW UZ-N77 & $\mathrm{M}^{*}$ & & & 104 & Fran Ridge & $\mathrm{M}^{*}$ & & \\
\hline 35 & USW UZ-N26 & .33 & $3 / 30$ & & 70 & N77 4" Gage & $\mathrm{M}^{*}$ & & & 104 & Fran Ridge (8") & $\mathrm{NI}$ & & \\
\hline 36 & USW UZ-N27 & .37 & $3 / 30$ & & 71 & USW UZ-N78 & $\mathrm{M}^{*}$ & & & 105 & Plug Hill & $\mathrm{M}^{*}$ & & \\
\hline 36 & N27 8" Gage & NI & & & 72 & USW UZ-N79 & $\mathrm{M}^{*}$ & & & 106 & 4JA (8") & NI & & \\
\hline 37 & USW UZ-N35 & $\mathrm{NI}$ & & & 73 & USW UZ-N80 & $\mathbf{M}^{*}$ & & & 107 & Knothead Gap & NI & & \\
\hline 38 & USW UZ-N36 & $\mathrm{NI}$ & & & 73 & N80 4" Gage & $\mathbf{M}^{*}$ & & & 108 & Upper Forty Mile & $\mathrm{NI}$ & & \\
\hline
\end{tabular}


Table 2. Summary of precipitation data from the nonrecording gage network at Yucca Mountain, Nevada, for water years 1992 and 1993 --Continued

\begin{tabular}{|c|c|c|c|c|c|c|c|c|c|c|c|c|c|c|}
\hline $\begin{array}{l}\text { Loca- } \\
\text { tion } \\
\text { code }\end{array}$ & $\begin{array}{c}\text { Site } \\
\text { nomenclature }\end{array}$ & $\begin{array}{l}\text { Amount } \\
\text { (Inches) }\end{array}$ & $\begin{array}{l}\text { Date } \\
\text { read }\end{array}$ & Notes & $\begin{array}{l}\text { Loca- } \\
\text { tion } \\
\text { code }\end{array}$ & $\begin{array}{c}\text { Site } \\
\text { nomenclature }\end{array}$ & $\begin{array}{l}\text { Amount } \\
\text { (inches) }\end{array}$ & $\begin{array}{l}\text { Date } \\
\text { read }\end{array}$ & Notes & $\begin{array}{l}\text { Loca- } \\
\text { tion } \\
\text { code }\end{array}$ & $\begin{array}{c}\text { Site } \\
\text { nomenclature }\end{array}$ & $\begin{array}{l}\text { Amount } \\
\text { (inches) }\end{array}$ & $\begin{array}{l}\text { Date } \\
\text { read }\end{array}$ & Notes \\
\hline \multicolumn{15}{|c|}{ STORM OF MARCH 28-31, 1992} \\
\hline 1 & UE-25 UZN \#1 & .78 & $4 / 7$ & & 39 & USW UZ-N37 & .71 & $4 / 8$ & & 74 & USW UZ-N81 & 1.23 & $4 / 2$ & $* *$ \\
\hline 2 & UE-25 UZN \#2 & .79 & $4 / 7$ & & 39 & N37 4" Gage & NI & & & 75 & USW UZ-N82 & 1.13 & $4 / 2$ & $* *$ \\
\hline 3 & UE-25 UZN \#3 & .78 & $4 / 7$ & & 40 & USW UZ-N40 & .75 & $4 / 6$ & & 75 & N82 4" Gage & 1.29 & $4 / 2$ & ** \\
\hline 4 & UE-25 UZN \#4 & .76 & $4 / 7$ & & 40 & N40 4" Gage & NI & & & 76 & USW UZ-N83 & 1.25 & $4 / 2$ & ** \\
\hline 4 & N4 4" Gage & NI & & & 41 & USW UZ-N41 & .79 & $4 / 2$ & & 77 & USW UZ-N84 & 1.16 & $4 / 2$ & \\
\hline 5 & UE-25 UZN \#5 & .79 & $4 / 7$ & & 42 & USW UZ-N42 & .79 & $4 / 6$ & & 78 & USW UZ-N86 & 1.28 & $4 / 2$ & $* *$ \\
\hline 6 & UE-25 UZN \#6 & .76 & $4 / 7$ & & 43 & USW UZ-N43 & .78 & $4 / 6$ & & 79 & USW UZ-N87 & 1.18 & $4 / 2$ & ** \\
\hline 7 & UE-25 UZN \#7 & .79 & $4 / 7$ & & 43 & N43 4" Gage & NI & & & 80 & USW UZ-N88 & 1.20 & $4 / 2$ & ** \\
\hline 8 & UE-25 UZN \#8 & .76 & $4 / 7$ & & 44 & USW UZ-N44 & .85 & $4 / 6$ & & 81 & USW UZ-N89 & 1.17 & $4 / 2$ & ** \\
\hline 9 & UE-25 UZN & .74 & $4 / 7$ & & 45 & USW UZ-N45 & .78 & $4 / 6$ & & 82 & USW UZ-N90 & 1.18 & $4 / 2$ & $* *$ \\
\hline 10 & UE-25 UZN \#10 & .76 & $4 / 7$ & & 46 & USW UZ-N46 & .77 & $4 / 6$ & & 82 & N90 4" Gage & 1.35 & $4 / 2$ & $* *$ \\
\hline 10 & N10 4" Gage & NI & & & 47 & USW UZ-N47 & .73 & $4 / 6$ & & 83 & USW UZ-N93 & .79 & $3 / 30$ & \\
\hline 11 & UE-25 UZN \#12 & .70 & $4 / 7$ & & 48 & USW UZ-N48 & 1.00 & $4 / 7$ & $* *$ & 84 & USW UZ-N94 & .83 & $3 / 30$ & \\
\hline 12 & UE-25 UZN \#13 & .81 & $4 / 7$ & & 49 & USW UZ-N49 & .97 & $4 / 7$ & $* *$ & 85 & USW UZ-N95 & .85 & $3 / 30$ & \\
\hline 13 & UE-25 UZN \#14 & .66 & $4 / 7$ & & so & USW UZ-N50 & .96 & $4 / 7$ & $* *$ & 86 & USW UZ-N96 & $\mathbf{M}$ & $3 / 30$ & \\
\hline 14 & UE-25 UZN \#18 & .73 & $4 / 6$ & & 50 & N50 4" Gage & NI & & & 87 & USW UZ-N98 & .97 & $3 / 30$ & \\
\hline 15 & UE-25 UZN \#19 & .78 & $4 / 6$ & & 51 & USW UZ-N51 & .95 & $4 / 7$ & $* *$ & 87 & N98 4" Gage & NI & & \\
\hline 16 & UE-25 UZN \#20 & .79 & $4 / 6$ & & 52 & USW UZ-N52 & .98 & $4 / 7$ & $m *$ & 88 & USW UZ-13 & .83 & $3 / 30$ & \\
\hline 17 & UE-25 UZN \#21 & .73 & $4 / 6$ & & 53 & USW UZ-N53 & 1.02 & $4 / 7$ & $* *$ & 88 & UZ-13 4" Gage & .92 & $3 / 30$ & \\
\hline 17 & N21 4" Gage & NI & & & 54 & USW UZ-N54 & $\mathbf{M}$ & & & 89 & USW GA-1 & .80 & $4 / 7$ & \\
\hline 18 & UE-25 UZN \#22 & .75 & $4 / 6$ & & 54 & N54 4" Gage & NI & & & 90 & USW G-2 & .83 & $4 / 7$ & \\
\hline 19 & UE-25 UZN \#23 & .67 & $4 / 6$ & & 55 & USW UZ-N55 & .94 & $4 / 7$ & $* *$ & 91 & UE25 WT-4 & .77 & $4 / 7$ & \\
\hline 20 & UE-25 UZN \#28 & .73 & $4 / 6$ & & 56 & USW UZ-N57 & .84 & $3 / 30$ & & 92 & UE25 WT-18 & .70 & $4 / 7$ & \\
\hline 21 & UE-25 UZN \#29 & .64 & $4 / 6$ & & 57 & USW UZ-N64 & NI & & & 93 & USW H-5 & 1.07 & $3 / 30$ & \\
\hline 22 & UE-25 UZN \#30 & .62 & $4 / 6$ & & 58 & USW UZ-N65 & .66 & $3 / 30$ & & 94 & HRF Wedge & .53 & $4 / 2$ & \\
\hline
\end{tabular}


Table 2. Summary of precipitation data from the nonrecording gage network at Yucca Mountain, Nevada, for water years 1992 and $1993--$ Continued

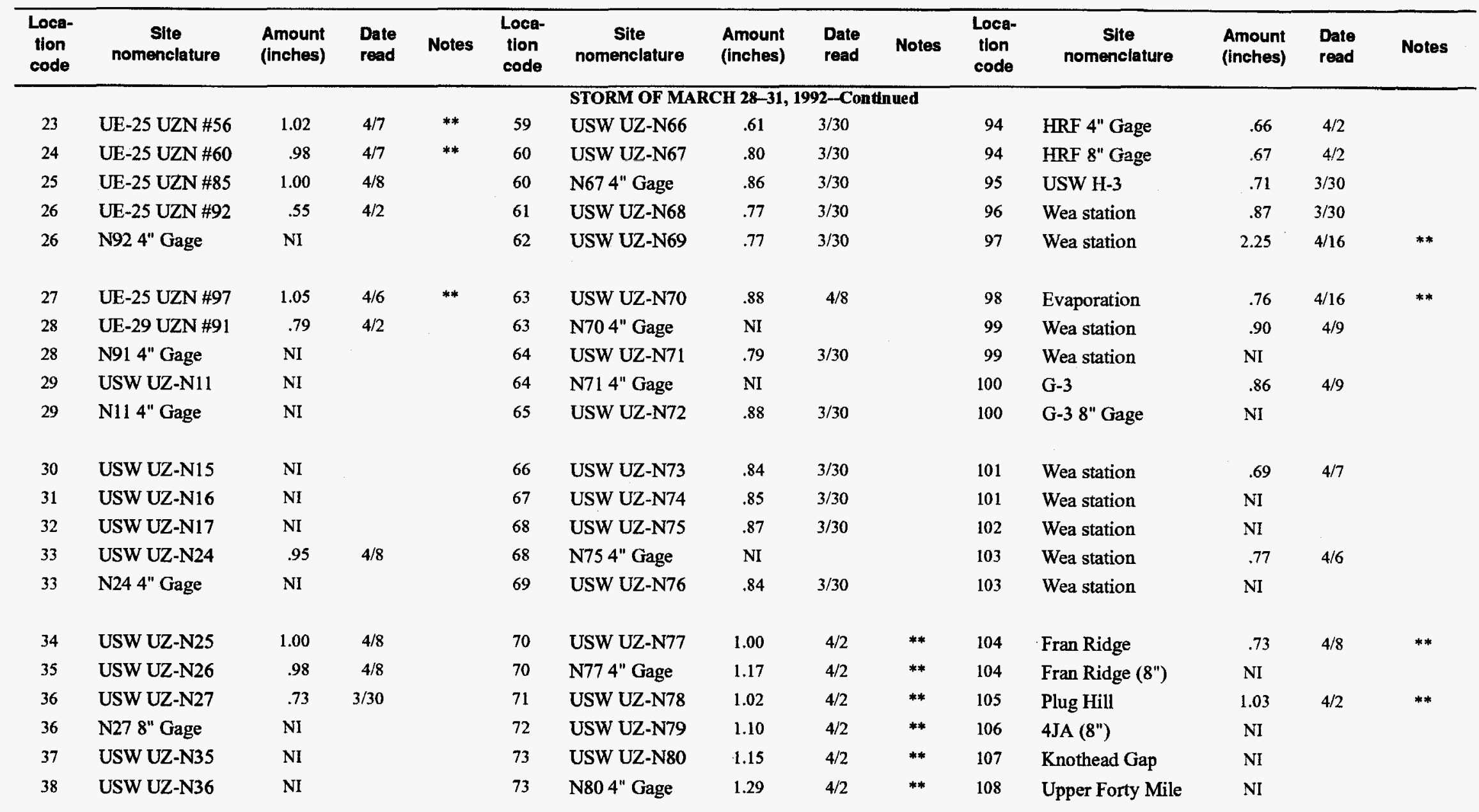


Table 2. Summary of precipitation data from the nonrecording gage network at Yucca Mountain, Nevada, for water years 1992 and 1993 --Continued

\begin{tabular}{|c|c|c|c|c|c|c|c|c|c|c|c|c|c|c|}
\hline $\begin{array}{l}\text { Loce- } \\
\text { tion } \\
\text { code }\end{array}$ & $\begin{array}{c}\text { Site } \\
\text { nomenclature }\end{array}$ & $\begin{array}{l}\text { Amount } \\
\text { (inches) }\end{array}$ & $\begin{array}{l}\text { Dale } \\
\text { read }\end{array}$ & Notes & $\begin{array}{l}\text { Loca- } \\
\text { tion } \\
\text { code }\end{array}$ & $\begin{array}{c}\text { Site } \\
\text { nomenclature }\end{array}$ & $\begin{array}{l}\text { Amount } \\
\text { (Inches) }\end{array}$ & $\begin{array}{l}\text { Date } \\
\text { read }\end{array}$ & Notes & $\begin{array}{l}\text { Loca- } \\
\text { tion } \\
\text { code }\end{array}$ & $\begin{array}{c}\text { Site } \\
\text { nomenclature }\end{array}$ & $\begin{array}{l}\text { Amount } \\
\text { (inches) }\end{array}$ & $\begin{array}{l}\text { Date } \\
\text { read }\end{array}$ & Notes \\
\hline \multicolumn{15}{|c|}{ STORM OF MAY 6, 1992} \\
\hline 1 & UE-25 UZN \#1 & 0 & $5 / 8$ & & 39 & USW UZ-N37 & 0 & $5 / 8$ & & 74 & USW UZ-N81 & 0 & $5 / 8$ & \\
\hline 2 & UE-25 UZN \#2 & 0 & $5 / 8$ & & 39 & N37 4" Gage & NI & & & 75 & USW UZ-N82 & 0 & $5 / 8$ & \\
\hline 3 & UE-25 UZN \#3 & 0 & $5 / 8$ & & 40 & USW UZ-N40 & 0 & $5 / 8$ & & 75 & N82 4" Gage & & & Removed \\
\hline 4 & UE-25 UZN \#4 & 0 & $5 / 8$ & & 40 & N40 4" Gage & NI & & & 76 & USW UZ-N83 & 0 & $5 / 8$ & \\
\hline 4 & N4 4" Gage & NI & & & 41 & USW UZ-N41 & 0 & $5 / 8$ & & 77 & USW UZ-N84 & 0 & $5 / 8$ & \\
\hline 5 & UE-25 UZN \#5 & 0 & $5 / 8$ & & 42 & USW UZ-N42 & 0 & $5 / 8$ & & 78 & USW UZ-N86 & 0 & $5 / 8$ & \\
\hline 6 & UE-25 UZN \#6 & 0 & $5 / 8$ & & 43 & USW UZ-N43 & 0 & $5 / 8$ & & 79 & USW UZ-N87 & 0 & $5 / 8$ & \\
\hline 7 & UE-25 UZN \#7 & 0 & $5 / 8$ & & 43 & N43 4" Gage & NI & & & 80 & USW UZ-N88 & 0 & $5 / 8$ & \\
\hline 8 & UE-25 UZN \#8 & 0 & $5 / 8$ & & 44 & USW UZ-N44 & 0 & $5 / 8$ & & 81 & USW UZ-N89 & 0 & $5 / 8$ & \\
\hline 9 & UE-25 UZN \#9 & 0 & $5 / 8$ & & 45 & USW UZ-N45 & 0 & $5 / 8$ & & 82 & USW UZ-N90 & 0 & $5 / 8$ & \\
\hline 10 & UE-25 UZN \#10 & 0 & $5 / 8$ & & 46 & USW UZ-N46 & 0 & $5 / 8$ & & 82 & N90 4" Gage & 0 & $5 / 8$ & \\
\hline 10 & N10 4" Gage & $\mathrm{NI}$ & & & 47 & USW UZ-N47 & 0 & $5 / 8$ & & 83 & USW UZ-N93 & 0 & $5 / 8$ & \\
\hline 11 & UE-25 UZN \#12 & 0 & $5 / 8$ & & 48 & USW UZ-N48 & 0 & $5 / 8$ & & 84 & USW UZ-N94 & 0 & $5 / 8$ & \\
\hline 12 & UE-25 UZN \#13 & 0 & $5 / 8$ & & 49 & USW UZ-N49 & 0 & $5 / 8$ & & 85 & USW UZ-N95 & 0 & $5 / 8$ & \\
\hline 13 & UE-25 UZN \#14 & 0 & $5 / 8$ & & 50 & USW UZ-N50 & 0 & $5 / 8$ & & 86 & USW UZ-N96 & 0 & $5 / 8$ & \\
\hline 14 & UE-25 UZN \#18 & 0 & $5 / 8$ & & 50 & N50 4" Gage & NI & & & 87 & USW UZ-N98 & 0 & $5 / 8$ & \\
\hline 15 & UE-25 UZN \#19 & 0 & $5 / 8$ & & 51 & USW UZ-N51 & 0 & $5 / 8$ & & 87 & N98 4" Gage & NI & & \\
\hline 16 & UE-25 UZN \#20 & 0 & $5 / 8$ & & 52 & USW UZ-N52 & 0 & $5 / 8$ & & 88 & USW UZ-13 & .02 & $5 / 8$ & \\
\hline 17 & UE-25 UZN \#21 & 0 & $5 / 8$ & & 53 & USW UZ-N53 & 0 & $5 / 8$ & & 88 & UZ-13 4" Gage & .06 & $5 / 8$ & \\
\hline 17 & N21 4" Gage & NI & & & 54 & USW UZ-N54 & 0 & $5 / 8$ & & 89 & USW GA-1 & 0 & $5 / 8$ & \\
\hline 18 & UE-25 UZN \#22 & 0 & $5 / 8$ & & 54 & N54 4" Gage & 0 & $5 / 8$ & & 90 & USW G-2 & 0 & $5 / 8$ & \\
\hline 19 & UE-25 UZN \#23 & 0 & $5 / 8$ & & 55 & USW UZ-N55 & 0 & $5 / 8$ & @ & 91 & UE25 WT-4 & 0 & $5 / 8$ & \\
\hline 20 & UE-25 UZN \#28 & 0 & $5 / 8$ & & 56 & USW UZ-N57 & 0 & $5 / 8$ & & 92 & UE25 WT-18 & 0 & $5 / 8$ & \\
\hline 21 & UE-25 UZN \#29 & 0 & $5 / 8$ & & 57 & USW UZ-N64 & NI & & & 93 & USW H-5 & .03 & $5 / 8$ & \\
\hline 22 & UE-25 UZN \#30 & 0 & $5 / 8$ & & 58 & USW UZ-N65 & 0 & $5 / 8$ & & 94 & HRF Wedge & 0 & $5 / 8$ & \\
\hline
\end{tabular}




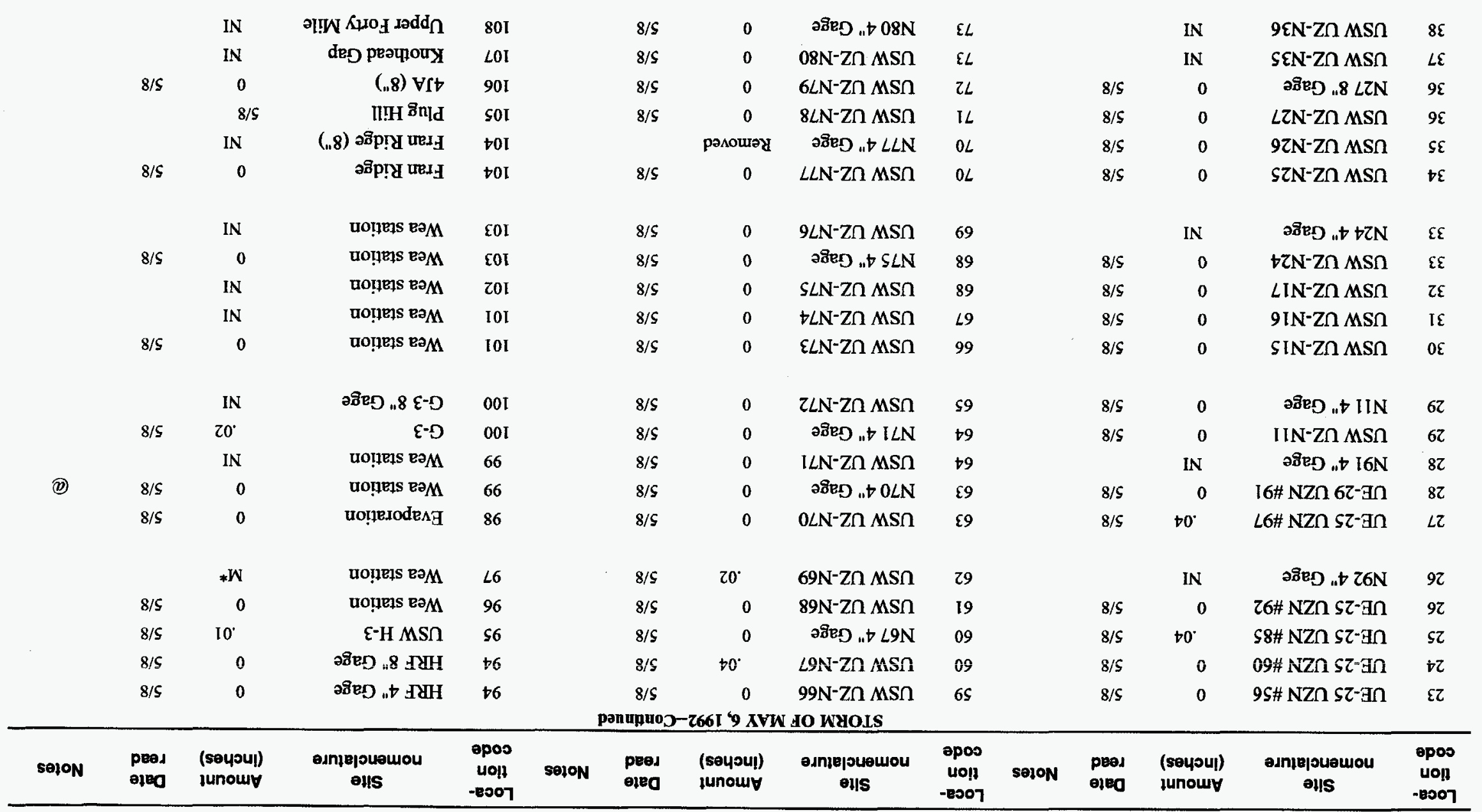

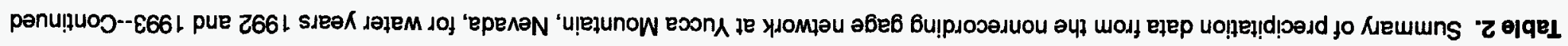


Table 2. Summary of precipitation data from the nonrecording gage network at Yucca Mountain, Nevada, for water years 1992 and $1993--$ Continued

\begin{tabular}{|c|c|c|c|c|c|c|c|c|c|c|c|c|c|c|}
\hline $\begin{array}{l}\text { Loca- } \\
\text { tion } \\
\text { code }\end{array}$ & $\begin{array}{c}\text { Site } \\
\text { nomenclature }\end{array}$ & $\begin{array}{l}\text { Amount } \\
\text { (inches) }\end{array}$ & $\begin{array}{l}\text { Date } \\
\text { read }\end{array}$ & Notes & $\begin{array}{l}\text { Loca- } \\
\text { tlon } \\
\text { code }\end{array}$ & $\begin{array}{c}\text { Site } \\
\text { nomenclature }\end{array}$ & $\begin{array}{l}\text { Amount } \\
\text { (inches) }\end{array}$ & $\begin{array}{l}\text { Date } \\
\text { read }\end{array}$ & Notes & $\begin{array}{l}\text { Loce- } \\
\text { tlon } \\
\text { code }\end{array}$ & $\begin{array}{c}\text { Site } \\
\text { nomenclature }\end{array}$ & $\begin{array}{l}\text { Amount } \\
\text { (inches) }\end{array}$ & $\begin{array}{l}\text { Date } \\
\text { read }\end{array}$ & Notes \\
\hline \multicolumn{15}{|c|}{ STORM OF MAY 8, 1992} \\
\hline 1 & UE-25 UZN\#1 & .05 & $5 / 11$ & & 39 & USW UZ-N37 & .08 & $5 / 11$ & & 74 & USW UZ-N8I & .11 & $5 / 15$ & \\
\hline 2 & $\mathrm{UE}-25 \mathrm{UZN} \# 2$ & .07 & $5 / 11$ & & 39 & N37 4" Gage & NI & & & 75 & USW UZ-N82 & .15 & $5 / 15$ & \\
\hline 3 & UE-25 UZN \#3 & .06 & $5 / 11$ & & 40 & USW UZ-N40 & .09 & $5 / 11$ & & 75 & N82 4" Gage & & & Removed \\
\hline 4 & UE-25 UZN \#4 & .05 & $5 / 11$ & & 40 & N40 4" Gage & NI & & & 76 & USW UZ-N83 & .12 & $5 / 15$ & \\
\hline 4 & N4 4" Gage & NI & & & 41 & USW UZ-N41 & .09 & $5 / 11$ & & 77 & USW UZ-N84 & .12 & $5 / 15$ & \\
\hline 5 & UE-25 UZN \#5 & .05 & $5 / 11$ & & 42 & USW UZ-N42 & .09 & $5 / 11$ & & 78 & USW UZ-N86 & $\mathbf{M}$ & $5 / 15$ & \\
\hline 6 & UE-25 UZN \#6 & .05 & $5 / 11$ & & 43 & USW UZ-N43 & .09 & $5 / 11$ & & 79 & USW UZ-N87 & .13 & $5 / 15$ & \\
\hline 7 & UE-25 UZN \#7 & .05 & $5 / 11$ & & 43 & N43 4" Gage & NI & & & 80 & USW UZ-N88 & .11 & $5 / 15$ & \\
\hline 8 & UE-25 UZN \#8 & .04 & $5 / 11$ & & 44 & USW UZ-N44 & .10 & $5 / 11$ & & 81 & USW UZ-N89 & .11 & $5 / 15$ & \\
\hline 9 & UE-25 UZN \#9 & .04 & $5 / 11$ & & 45 & USW UZ-N45 & .10 & $5 / 11$ & & 82 & USW UZ-N90 & .13 & $5 / 15$ & \\
\hline 10 & UE-25 UZN \#10 & .05 & $5 / 11$ & & 46 & USW UZ-N46 & .06 & $5 / 11$ & & 82 & N90 4" Gage & .16 & $5 / 15$ & \\
\hline 10 & N10 4" Gage & NI & & & 47 & USW UZ-N47 & .08 & $5 / 11$ & & 83 & USW UZ-N93 & .09 & $5 / 11$ & \\
\hline 11 & UE-25 UZN \#12 & .05 & $5 / 11$ & & 48 & USW UZ-N48 & .04 & $5 / 12$ & & 84 & USW UZ-N94 & .08 & $5 / 11$ & \\
\hline 12 & UE-25 UZN \#13 & .05 & $5 / 11$ & & 49 & USW UZ-N49 & .07 & $5 / 12$ & & 85 & USW UZ-N95 & .10 & $5 / 11$ & \\
\hline 13 & UE-25 UZN \#14 & .03 & $5 / 11$ & & 50 & USW UZ-N50 & .04 & $5 / 12$ & & 86 & USW UZ-N96 & .08 & $5 / 11$ & \\
\hline 14 & UE-25 UZN \#18 & .07 & $5 / 11$ & & 50 & N50 4" Gage & NI & & & 87 & USW UZ-N98 & .15 & $5 / 11$ & \\
\hline 15 & UE-25 UZN \#19 & .07 & $5 / 12$ & & 51 & USW UZ-N51 & .10 & $5 / 12$ & & 87 & N98 4" Gage & NI & & \\
\hline 16 & UE-25 UZN \#20 & .07 & $5 / 12$ & & 52 & USW UZ-N52 & .05 & $5 / 12$ & & 88 & USW UZ-13 & .08 & $5 / 11$ & \\
\hline 17 & UE-25 UZN \#21 & .08 & $5 / 12$ & & 53 & USW UZ-N53 & .08 & $5 / 12$ & & 88 & UZ-13 4" Gage & .11 & $5 / 11$ & \\
\hline 17 & N21 4" Gage & NI & & & 54 & USW UZ-N54 & .08 & $5 / 12$ & & 89 & USW GA-1 & .09 & $5 / 11$ & \\
\hline 18 & UE-25 UZN \#22 & .08 & $5 / 12$ & & 54 & N54 4" Gage & .12 & $5 / 12$ & & 90 & USW G-2 & .07 & $5 / 11$ & \\
\hline 19 & UE-25 UZN \#23 & .08 & $5 / 12$ & & 55 & USW UZ-N55 & .12 & $5 / 12$ & & 91 & UE25 WT-4 & .01 & $5 / 11$ & \\
\hline 20 & UE-25 UZN \#28 & .07 & $5 / 12$ & & 56 & USW UZ-N57 & .07 & $5 / 11$ & & 92 & UE25 WT-18 & $\mathbf{M}^{*}$ & & \\
\hline 21 & UE-25 UZN \#29 & .08 & $5 / 12$ & & 57 & USW UZ-N64 & NI & & & 93 & USW H-5 & .14 & $5 / 11$ & \\
\hline 22 & UE-25 UZN \#30 & .07 & $5 / 12$ & & 58 & USW UZ-N65 & .04 & $5 / 11$ & & 94 & HRF Wedge & .01 & $5 / 11$ & \\
\hline
\end{tabular}




\begin{tabular}{|c|c|c|c|c|c|c|c|c|c|c|c|c|c|c|}
\hline & & IN & 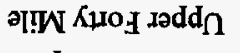 & $80 I$ & & $\mathrm{sI} / \mathrm{s}$ & W & วชีอด „† 08N & $\varepsilon L$ & & II $/ \mathbf{S}$ & $90^{\circ}$ & 9EN-Zn MSก & $8 \mathcal{E}$ \\
\hline & & IN & deD peaqpouY & LOI & & 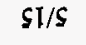 & $\mathrm{OI}^{\circ}$ & $08 \mathrm{~N}-\mathrm{Zn} \mathrm{MS} \Omega$ & $\varepsilon L$ & & & IN & SEN-Zn MSO & $L \varepsilon$ \\
\hline & $\mathrm{II} / \mathrm{S}$ & $10^{\circ}$ & $(.8) \mathrm{VSt}$ & $90 \mathrm{I}$ & & $\mathfrak{S l} / \mathfrak{S}$ & $\angle I^{\circ}$ & 6LN-Zח MS & $Z L$ & & $11 / 5$ & or & วฮีeD „8 LZN & $9 \varepsilon$ \\
\hline & $\mathbf{s l} / \mathbf{s}$ & $L Z^{*}$ & II!H Iีnld & \$0I & & $\boldsymbol{S l} / \boldsymbol{S}$ & $60^{\circ}$ & 8LN-Zח MSO & IL & & $\mathrm{Il} / \mathrm{S}$ & $80^{\circ}$ & $\angle Z N-Z \cap M S \cap$ & $9 \varepsilon$ \\
\hline & & IN & 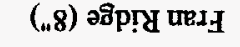 & tol & & & рәлотеу & 28BED „t & $0 L$ & & $I I / S$ & $\mathrm{II}^{*}$ & 9ZN-Zn MSn & 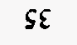 \\
\hline & $\varepsilon \mathrm{L} / \mathrm{S}$ & $90^{\circ}$ & әิేp!ู นR. & tol & & $S \mathbf{I} / \mathbf{S}$ & 纟I & $\angle L N^{-} \mathrm{Z} \cap \mathrm{MS} \cap$ & $0 L$ & & II/S & $8 I^{\circ}$ & SZN-ZO MSO & $\downarrow \varepsilon$ \\
\hline & & IN & uoṭp]s вәM & £0I & & $\mathrm{II} / \mathrm{S}$ & $80^{\circ}$ & $9 L N-\mathrm{Z} \cap \mathrm{MS} \cap$ & 69 & & & IN & 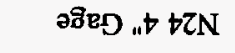 & $\varepsilon \varepsilon$ \\
\hline & $I I / S$ & $90^{\circ}$ & uoțpęs ваM & E0I & & $\mathrm{II} / \mathrm{s}$ & $\varepsilon \tau^{\circ}$ & $28 \mathrm{eD}$ „ $\$ S L N$ & 89 & & $I I / S$ & so & $\nabla Z N^{-} \mathrm{Z} \cap \mathrm{MS} \Omega$ & $\mathfrak{E} \mathfrak{E}$ \\
\hline & & IN & uoṭpes вам & Z0I & & $I I / \varsigma$ & $90^{\circ}$ & SLN-Zח MS & 89 & & $\mathrm{II} / \mathrm{S}$ & $\mathrm{II}^{*}$ & $\angle I N-Z \cap M S \cap$ & $z \varepsilon$ \\
\hline & & IN & uo!̣pels вәM & IOI & & $I I / S$ & $80^{\circ}$ & $\nabla L N^{-Z} \mathrm{Zn} M S \cap$ & $\angle 9$ & & It/S & II & $9 \mathrm{IN}-\mathrm{Z} \cap \mathrm{MS} \Omega$ & Iย \\
\hline & $2 \mathrm{I} / \mathrm{S}$ & II $^{\circ}$ & UO!̣E]S RכM & IOI & & $I t / \mathrm{s}$ & $\mathrm{OI}^{\circ}$ & $\mathcal{E} L \mathrm{~N}-\mathrm{Zn} \mathrm{MS} \cap$ & 99 & & $\mathrm{II} / \mathrm{S}$ & II' & SIN-ZA MSO & $0 \mathcal{E}$ \\
\hline & & IN & 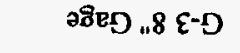 & $00 t$ & & $I t / S$ & $60^{\circ}$ & ZLN-Zn MS & $\$ 9$ & & $\mathrm{II} / \mathrm{S}$ & so & əชิeD „t IIN & 62 \\
\hline & $I I / \varsigma$ & $80^{\circ}$ & $\varepsilon-D$ & $00 \mathrm{t}$ & & $I I / S$ & $\varepsilon \digamma^{\circ}$ & $\partial 8 ื \mathrm{ED} \ldots I L N$ & t9 & & $11 / \mathrm{s}$ & 0 & IIN-Zn MSO & 62 \\
\hline & & IN & 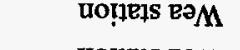 & 66 & & $\mathrm{II} / \mathrm{S}$ & $60^{\circ}$ & I LN-Zn MSח & $t 9$ & & & IN & ว8ีeD „† I6N & 82 \\
\hline & $I I / S$ & $\mathrm{ZI}$ & uo!̣teIs EәM & 66 & & $\mathrm{II} / \mathrm{S}$ & $\angle I^{\circ}$ & əoีeD „ & $\varepsilon 9$ & & $\mathfrak{E I / S}$ & $\mathrm{zI}$ & I6\# NZn 62-3n & 82 \\
\hline & $\mathrm{II} / \mathrm{S}$ & 0 & นо!̣EIOdеАЯ & 86 & & $\mathrm{II} / \mathrm{S}$ & $\mathrm{ZI}^{\circ}$ & $0 \angle N^{-} Z \cap M S \cap$ & $\varepsilon 9$ & & $I I / S$ & $z \mathbf{l}^{*}$ & L6\# NZn ऽZ-Gก & $\angle Z$ \\
\hline & & $* \mathbf{W}$ & uo!̣E]S EәM & $\angle 6$ & & $I t / S$ & $\Delta I^{\circ}$ & $69 \mathrm{~N}^{-} \mathrm{Z} \cap \mathrm{MS} \cap$ & 29 & & & IN & 28ีED „ & 92 \\
\hline & $\mathrm{II} / \mathrm{S}$ & $80^{\circ}$ & UO!̣EIS ROM & 96 & & $\mathrm{II} / \mathrm{S}$ & $6 \mathrm{I}^{\circ}$ & $89 N^{-} Z \cap M S \cap$ & I9 & & $\mathfrak{\varepsilon I / \mathcal { S }}$ & $z 0^{\circ}$ & 乙6\# NZn sZ-An & 92 \\
\hline & $\mathrm{II} / \mathrm{s}$ & $80^{\circ}$ & $\varepsilon-H M S \cap$ & \6 & & $\mathrm{II} / \mathrm{S}$ & $\mathfrak{E Z}$ & 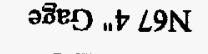 & 09 & & $\mathrm{It} / \mathrm{S}$ & हI' & $s 8 \# N Z \cap \varsigma Z-\exists \cap$ & $s \mathcal{L}$ \\
\hline & $\mathrm{II} / \mathrm{S}$ & $\mathbf{W}$ & 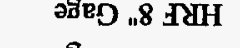 & $t 6$ & & $\mathrm{II} / \mathrm{S}$ & SI & $\angle 9 N-Z \cap M S \cap$ & 09 & & $Z \mathbf{I} / \mathrm{S}$ & ؟I' & 09\# NZח sZ- In & $t z$ \\
\hline & $\mathrm{II} / \mathrm{s}$ & E0 & 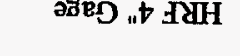 & 16 & & II $/ \mathrm{S}$ & $\$ 0^{\circ}$ & $99 N^{-} \mathrm{Z} \cap \mathrm{MS} \Omega$ & $6 S$ & & $\tau \mathbf{I} / \mathbf{S}$ & IN & 9S\# NZn SZ-تП & $\varepsilon \imath$ \\
\hline Selon & $\begin{array}{l}\text { pred } \\
\text { eqea }\end{array}$ & $\begin{array}{l}\text { (seyoul) } \\
\text { funoury }\end{array}$ & $\begin{array}{c}\text { OAnIEjOLEWOU } \\
\text { OIIS }\end{array}$ & $\begin{array}{l}\text { epos } \\
\text { U0!! } \\
-8007\end{array}$ & Solon & $\begin{array}{l}\text { peed } \\
\text { eled }\end{array}$ & $\begin{array}{l}\text { (seyou) } \\
\text { junowy }\end{array}$ & $\begin{array}{c}\text { e.mpe|จuewou } \\
\text { el|s }\end{array}$ & $\begin{array}{l}\text { өpos } \\
\text { uo|l } \\
-8007\end{array}$ & Selon & $\begin{array}{l}\text { pead } \\
\text { elea }\end{array}$ & $\begin{array}{l}\text { (soyou!) } \\
\text { Iunowy }\end{array}$ & 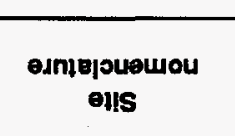 & $\begin{array}{c}\text { opos } \\
\text { 40|1 } \\
-8007\end{array}$ \\
\hline
\end{tabular}

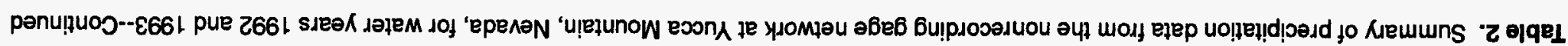


Table 2. Summary of precipitation data from the nonrecording gage network at Yucca Mountain, Nevada, for water years 1992 and $1993--$ Continued

\begin{tabular}{|c|c|c|c|c|c|c|c|c|c|c|c|c|c|c|}
\hline $\begin{array}{l}\text { Loca- } \\
\text { tion } \\
\text { code }\end{array}$ & $\begin{array}{c}\text { Site } \\
\text { nomenclature }\end{array}$ & $\begin{array}{l}\text { Amount } \\
\text { (inches) }\end{array}$ & $\begin{array}{l}\text { Date } \\
\text { read }\end{array}$ & Notes & $\begin{array}{l}\text { Loca- } \\
\text { tion } \\
\text { code }\end{array}$ & $\begin{array}{c}\text { Site } \\
\text { nomenclature }\end{array}$ & $\begin{array}{l}\text { Amount } \\
\text { (Inches) }\end{array}$ & $\begin{array}{l}\text { Date } \\
\text { read }\end{array}$ & Notes & $\begin{array}{l}\text { Loca- } \\
\text { tion } \\
\text { code }\end{array}$ & $\begin{array}{c}\text { Site } \\
\text { nomenclature }\end{array}$ & $\begin{array}{l}\text { Amount } \\
\text { (inches) }\end{array}$ & $\begin{array}{l}\text { Date } \\
\text { read }\end{array}$ & Notes \\
\hline \multicolumn{15}{|c|}{ STORM OF MAY $21 \& 26,1992$} \\
\hline 1 & UE-25 UZN \#1 & .05 & $6 / 16$ & & 39 & USW UZ-N37 & .04 & $6 / 30$ & & 74 & USW UZ-N81 & .01 & $6 / 23$ & \\
\hline 2 & UE-25 UZN \#2 & .17 & $6 / 16$ & & 39 & N37 4" Gage & NI & & & 75 & USW UZ-N82 & .02 & $6 / 23$ & \\
\hline 3 & UE-25 UZN \#3 & .11 & $6 / 16$ & & 40 & USW UZ-N40 & .02 & $6 / 30$ & & 75 & N82 4" Gage & & & Removed \\
\hline 4 & UE-25 UZN \#4 & .08 & $6 / 16$ & & 40 & N40 4" Gage & NI & & & 76 & USW UZ-N83 & .04 & $6 / 23$ & \\
\hline 4 & N4 4" Gage & NI & & & 41 & USW UZ-N41 & .02 & $6 / 30$ & & 77 & USW UZ-N84 & .03 & $6 / 23$ & \\
\hline 5 & UE-25 UZN \#5 & .09 & $6 / 16$ & & 42 & USW UZ-N42 & .02 & $6 / 30$ & & 78 & USW UZ-N86 & .08 & $6 / 23$ & \\
\hline 6 & UE-25 UZN \#6 & .10 & & & 43 & USW UZ-N43 & .03 & $6 / 30$ & & 79 & USW UZ-N87 & .05 & $6 / 23$ & \\
\hline 7 & UE-25 UZN \#7 & .08 & $6 / 16$ & & 43 & N43 4" Gage & $\mathrm{NI}$ & & & 80 & USW UZ-N88 & .06 & $6 / 23$ & \\
\hline 8 & UE-25 UZN \#8 & .09 & $6 / 16$ & & 44 & USW UZ-N44 & .03 & $6 / 30$ & & 81 & USW UZ-N89 & .02 & $6 / 23$ & \\
\hline 9 & UE-25 UZN \#9 & .09 & $6 / 16$ & & 45 & USW UZ-N45 & .02 & $6 / 30$ & & 82 & USW UZ-N90 & .03 & $6 / 23$ & \\
\hline 10 & UE-25 UZN \#10 & .05 & $6 / 16$ & & 46 & USW UZ-N46 & .08 & $7 / 1$ & & 82 & N90 4" Gage & .05 & $6 / 23$ & \\
\hline 10 & N10 4" Gage & $\mathrm{NI}$ & & & 47 & USW UZ-N47 & .08 & $6 / 30$ & & 83 & USW UZ-N93 & .03 & $7 / 8$ & \\
\hline 11 & UE-25 UZN \#12 & .10 & $6 / 16$ & & 48 & USW UZ-N48 & .03 & $7 / 1$ & & 84 & USW UZ-N94 & .02 & $7 / 8$ & \\
\hline 12 & UE-25 UZN \#13 & .22 & $6 / 16$ & & 49 & USW UZ-N49 & .03 & $7 / 1$ & & 85 & USW UZ-N95 & .03 & $7 / 8$ & \\
\hline 13 & UE-25 UZN \#14 & .11 & $6 / 16$ & & 50 & USW UZ-N50 & .01 & $7 / 1$ & & 86 & USW UZ-N96 & .03 & $7 / 8$ & \\
\hline 14 & UE-25 UZN \#18 & .02 & $6 / 30$ & & 50 & N50 4" Gage & NI & & & 87 & USW UZ-N98 & .07 & $6 / 30$ & \\
\hline 15 & UE-25 UZN \#19 & 0 & $7 / 1$ & & 51 & USW UZ-N51 & .02 & $7 / 1$ & & 87 & N98 4" Gage & NI & & \\
\hline 16 & UE-25 UZN \#20 & 0 & $7 / 1$ & & 52 & USW UZ-N52 & .02 & $7 / 1$ & & 88 & USW UZ-13 & 0 & $7 / 8$ & \\
\hline 17 & UE-25 UZN \#21 & 0 & $7 / 1$ & & 53 & USW UZ-N53 & .01 & $7 / 1$ & & 88 & UZ-13 4" Gage & .02 & $7 / 8$ & \\
\hline 17 & N21 4" Gage & NI & & & 54 & USW UZ-N54 & .02 & $7 / 1$ & & 89 & USW GA-1 & 0 & $6 / 16$ & \\
\hline 18 & UE-25 UZN \#22 & 0 & $7 / 1$ & & 54 & N54 4" Gage & .03 & $7 / 1$ & & 90 & USW G-2 & 0 & $6 / 16$ & \\
\hline 19 & UE-25 UZN \#23 & 0 & $7 / 1$ & & 55 & USW UZ-N55 & .04 & $7 / 1$ & & 91 & UE25 WT-4 & .13 & $6 / 16$ & \\
\hline 20 & UE-25 UZN \#28 & 0 & $7 / 1$ & & 56 & USW UZ-N57 & 0 & $7 / 8$ & & 92 & UE25 WT-18 & .10 & $6 / 16$ & $* *$ \\
\hline 21 & UE-25 UZN \#29 & 0 & $7 / 1$ & & 57 & USW UZ-N64 & NI & & & 93 & USW H-S & .05 & $7 / 8$ & \\
\hline 22 & UE-25 UZN \#30 & 0 & $7 / 1$ & & 58 & USW UZ-N65 & .01 & $7 / 8$ & & 94 & HRF Wedge & 0 & $5 / 26$ & \\
\hline
\end{tabular}


Table 2. Summary of precipitation data from the nonrecording gage network at Yucca Mountain, Nevada, for water years 1992 and $1993--$ Continued

\begin{tabular}{|c|c|c|c|c|c|c|c|c|c|c|c|c|c|c|}
\hline $\begin{array}{l}\text { Loca- } \\
\text { tlon } \\
\text { code }\end{array}$ & $\begin{array}{c}\text { Site } \\
\text { nomenelature }\end{array}$ & $\begin{array}{l}\text { Amount } \\
\text { (inches) }\end{array}$ & $\begin{array}{l}\text { Date } \\
\text { read }\end{array}$ & Notes & $\begin{array}{l}\text { Loca- } \\
\text { tion } \\
\text { code }\end{array}$ & $\begin{array}{c}\text { Site } \\
\text { nomenclature }\end{array}$ & $\begin{array}{l}\text { Amount } \\
\text { (inches) }\end{array}$ & $\begin{array}{l}\text { Date } \\
\text { read }\end{array}$ & Notes & $\begin{array}{l}\text { Loca- } \\
\text { tion } \\
\text { code }\end{array}$ & $\begin{array}{c}\text { Site } \\
\text { nomenclature }\end{array}$ & $\begin{array}{l}\text { Amount } \\
\text { (Inches) }\end{array}$ & $\begin{array}{l}\text { Date } \\
\text { read }\end{array}$ & Notes \\
\hline \multicolumn{15}{|c|}{ STORM OF MAY 21 \& 26, 1992-Continued } \\
\hline 23 & UE-25 UZN \#56 & 0 & $7 / 1$ & & 59 & USW UZ-N66 & 0 & $7 / 8$ & & 94 & HRF 4" Gage & 0 & $5 / 26$ & \\
\hline 24 & UE-25 UZN \#60 & 0 & $6 / 30$ & & 60 & USW UZ-N67 & 0 & $7 / 8$ & & 94 & HRF 8" Gage & $\mathrm{T}$ & $5 / 26$ & \\
\hline 25 & UE-25 UZN \#85 & .09 & $7 / 1$ & & 60 & N67 4" Gage & .02 & $7 / 8$ & & 95 & USW H-3 & 0 & $7 / 8$ & \\
\hline 26 & UE-25 UZN \#92 & .02 & $7 / 8$ & & 61 & USW UZ-N68 & 0 & $7 / 8$ & & 96 & Wea station & .02 & $7 / 8$ & \\
\hline 26 & N92 4" Gage & NI & & & 62 & USW UZ-N69 & .01 & $7 / 8$ & & 97 & Wea station & .12 & $7 / 8$ & $* *$ \\
\hline 27 & UE-25 UZN \#97 & $\mathbf{0}$ & $7 / 1$ & & 63 & USW UZ-N70 & .14 & $6 / 30$ & & 98 & Evaporation & .02 & 6/16 & \\
\hline 28 & UE-29 UZN \#91 & .14 & $7 / 8$ & & 63 & N70 4" Gage & .13 & $6 / 30$ & & 99 & Wea station & .04 & $7 / 8$ & \\
\hline 28 & N91 4" Gage & NI & & & 64 & USW UZ-N71 & .05 & $7 / 8$ & & 99 & Wea station & $\mathrm{NI}$ & & \\
\hline 29 & USW UZ-N11 & NI & $6 / 16$ & & 64 & N71 4" Gage & .06 & $7 / 8$ & & 100 & G-3 & .08 & $7 / 8$ & \\
\hline 29 & N11 4" Gage & 0 & $6 / 16$ & & 65 & USW UZ-N72 & .04 & $7 / 8$ & & 100 & G-3 8" Gage & NI & & \\
\hline 30 & USW UZ-N15 & .01 & $6 / 16$ & & 66 & USW UZ-N73 & .05 & $7 / 8$ & & 101 & Wea station & .03 & $6 / 30$ & \\
\hline 31 & USW UZ-N16 & 0 & $6 / 16$ & & 67 & USW UZ-N74 & .04 & $7 / 8$ & & 101 & Wea station & NI & & \\
\hline 32 & USW UZ-N17 & 0 & $6 / 16$ & & 68 & USW UZ-N75 & .03 & $7 / 8$ & & 102 & Wea station & NI & & \\
\hline 33 & USW UZ-N24 & .07 & $6 / 30$ & & 68 & N75 4" Gage & .05 & $7 / 8$ & & 103 & Wea station & .06 & $6 / 30$ & \\
\hline 33 & N24 4" Gage & NI & & & 69 & USW UZ-N76 & .04 & $7 / 8$ & & 103 & Wea station & NI & & \\
\hline 34 & USW UZ-N25 & .08 & $6 / 30$ & & 70 & USW UZ-N77 & .01 & $6 / 23$ & & 104 & Fran Ridge & .04 & $7 / 8$ & \\
\hline 35 & USW UZ-N26 & .08 & $6 / 30$ & & 70 & N77 4" Gage & Removed & & & 104 & Fran Ridge (8") & NI & & \\
\hline 36 & USW UZ-N27 & .04 & $7 / 8$ & & 71 & USW UZ-N78 & .01 & $6 / 23$ & & 105 & Plug Hill & .10 & $6 / 23$ & \\
\hline 36 & N27 8" Gage & .17 & $7 / 8$ & & 72 & USW UZ-N79 & .06 & $6 / 23$ & & 106 & $4 \mathrm{JA}(8 ")$ & .02 & $6 / 16$ & \\
\hline 37 & USW UZ-N35 & NI & & & 73 & USW UZ-N80 & .01 & $6 / 23$ & & 107 & Knothead Gap & NI & & \\
\hline 38 & USW UZ-N36 & .01 & $6 / 16$ & & 73 & N80 4" Gage & .05 & $6 / 23$ & & 108 & Upper Forty Mile & NI & & \\
\hline
\end{tabular}


Table 2. Summary of precipitation data from the nonrecording gage network at Yucca Mountain, Nevada, for water years 1992 and $1993-$. Continued

\begin{tabular}{|c|c|c|c|c|c|c|c|c|c|c|c|c|c|c|}
\hline $\begin{array}{l}\text { Loca- } \\
\text { tion } \\
\text { code }\end{array}$ & $\begin{array}{c}\text { Site } \\
\text { nomenclature }\end{array}$ & $\begin{array}{l}\text { Amount } \\
\text { (Inches) }\end{array}$ & $\begin{array}{l}\text { Date } \\
\text { read }\end{array}$ & Notes & $\begin{array}{l}\text { Loca- } \\
\text { tion } \\
\text { code }\end{array}$ & $\begin{array}{c}\text { Site } \\
\text { nomenclature }\end{array}$ & $\begin{array}{l}\text { Amount } \\
\text { (inches) }\end{array}$ & $\begin{array}{l}\text { Date } \\
\text { read }\end{array}$ & Notes & $\begin{array}{l}\text { Loca- } \\
\text { tion } \\
\text { code }\end{array}$ & $\begin{array}{c}\text { Site } \\
\text { nomenclature }\end{array}$ & $\begin{array}{l}\text { Amount } \\
\text { (inches) }\end{array}$ & $\begin{array}{l}\text { Date } \\
\text { read }\end{array}$ & Notes \\
\hline \multicolumn{15}{|c|}{ STORM OF JULY 12-13 \& 15, 1992} \\
\hline 1 & UE-25 UZN \#1 & 0 & $7 / 16$ & & 39 & USW UZ-N37 & 0 & $7 / 16$ & & 74 & USW UZ-N81 & 0 & $7 / 16$ & \\
\hline 2 & UE-25 UZN \#2 & 0 & $7 / 16$ & & 39 & N37 4" Gage & 0 & $7 / 16$ & & 75 & USW UZ-N82 & 0 & $7 / 16$ & \\
\hline 3 & UE-25 UZN \#3 & 0 & $7 / 16$ & & 40 & USW UZ-N40 & 0 & $7 / 16$ & & 75 & N82 4" Gage & & & Removed \\
\hline 4 & UE-25 UZN \#4 & 0 & $7 / 16$ & & 40 & N40 4" Gage & $\mathrm{NI}$ & & & 76 & USW UZ-N83 & 0 & $7 / 16$ & \\
\hline 4 & N4 4" Gage & NI & & & 41 & USW UZ-N41 & 0 & $7 / 16$ & & 77 & USW UZ-N84 & 0 & $7 / 16$ & \\
\hline 5 & UE-25 UZN \#5 & 0 & $7 / 16$ & & 42 & USW UZ-N42 & 0 & $7 / 16$ & & 78 & USW UZ-N86 & 0 & $7 / 16$ & \\
\hline 6 & UE-25 UZN \#6 & 0 & $7 / 16$ & & 43 & USW UZ-N43 & 0 & $7 / 16$ & & 79 & USW UZ-N87 & 0 & $7 / 16$ & \\
\hline 7 & UE-25 UZN \#7 & 0 & $7 / 16$ & & 43 & N43 4" Gage & NI & & & 80 & USW UZ-N88 & 0 & $7 / 16$ & \\
\hline 8 & UE-25 UZN \#8 & 0 & $7 / 16$ & & 44 & USW UZ-N44 & 0 & $7 / 16$ & & 81 & USW UZ-N89 & 0 & $7 / 16$ & \\
\hline 9 & UE-25 UZN \#9 & $\mathbf{0}$ & $7 / 16$ & & 45 & USW UZ-N45 & $\mathbf{0}$ & $7 / 16$ & & 82 & USW UZ-N90 & 0 & $7 / 16$ & \\
\hline 10 & UE-25 UZN \#10 & 0 & $7 / 16$ & & 46 & USW UZ-N46 & .02 & $7 / 16$ & & 82 & N90 4" Gage & 0 & $7 / 16$ & \\
\hline 10 & N10 4" Gage & NI & & & 47 & USW UZ-N47 & .01 & $7 / 16$ & & 83 & USW UZ-N93 & 0 & $7 / 16$ & \\
\hline 11 & UE-25 UZN \#12 & 0 & $7 / 16$ & & 48 & USW UZ-N48 & 0 & $7 / 16$ & & 84 & USW UZ-N94 & 0 & $7 / 16$ & \\
\hline 12 & UE-25 UZN \#13 & 0 & $7 / 16$ & & 49 & USW UZ-N49 & 0 & $7 / 16$ & & 85 & USW UZ-N95 & 0 & $7 / 16$ & \\
\hline 13 & UE-25 UZN \#14 & o & $7 / 16$ & & 50 & USW UZ-N50 & 0 & $7 / 16$ & & 86 & USW UZ-N96 & 0 & $7 / 16$ & \\
\hline 14 & UE-25 UZN \#18 & 0 & $7 / 16$ & & 50 & N50 4" Gage & NI & & & 87 & USW UZ-N98 & 0 & $7 / 16$ & \\
\hline 15 & UE-25 UZN \#19 & 0 & $7 / 16$ & & 51 & USW UZ-N51 & 0 & $7 / 16$ & & 87 & N98 4" Gage & NI & & \\
\hline 16 & UE-25 UZN \#20 & 0 & $7 / 16$ & & 52 & USW UZ-N52 & 0 & $7 / 16$ & & 88 & USW UZ-13 & 0 & $7 / 16$ & \\
\hline 17 & UE-25 UZN \#21 & 0 & $7 / 16$ & & 53 & USW UZ-N53 & 0 & $7 / 16$ & & 88 & UZ-13 4" Gage & .01 & $7 / 16$ & \\
\hline 17 & N21 4" Gage & NI & & & 54 & USW UZ-N54 & 0 & $7 / 16$ & & 89 & USW GA-1 & .13 & $7 / 16$ & \\
\hline 18 & UE-25 UZN \#22 & 0 & $7 / 16$ & & 54 & N54 4" Gage & 0 & $7 / 16$ & & 90 & USW G-2 & .14 & $7 / 16$ & \\
\hline 19 & UE-25 UZN \#23 & 0 & $7 / 16$ & & 55 & USW UZ-N55 & 0 & $7 / 16$ & & 91 & UE25 WT-4 & 0 & $7 / 16$ & \\
\hline 20 & UE-25 UZN \#28 & 0 & $7 / 16$ & & 56 & USW UZ-N57 & 0 & $7 / 16$ & & 92 & UE25 WT-18 & 0 & $7 / 16$ & \\
\hline 21 & UE-25 UZN \#29 & 0 & $7 / 16$ & & 57 & USW UZ-N64 & 0 & $7 / 16$ & & 93 & USW H-5 & 0 & $7 / 16$ & \\
\hline 22 & UE-25 UZN \#30 & 0 & $7 / 16$ & & 58 & USW UZ-N65 & 0 & $7 / 16$ & & 94 & HRF Wedge & 0 & $7 / 16$ & \\
\hline
\end{tabular}


Table 2. Summary of precipitation data from the nonrecording gage network at Yucca Mountain, Nevada, for water years 1992 and $1993--$ Continued

\begin{tabular}{|c|c|c|c|c|c|c|c|c|c|c|c|c|c|c|}
\hline $\begin{array}{l}\text { Loca- } \\
\text { tion } \\
\text { code }\end{array}$ & $\begin{array}{c}\text { Site } \\
\text { nomenclature }\end{array}$ & $\begin{array}{l}\text { Amount } \\
\text { (inches) }\end{array}$ & $\begin{array}{l}\text { Date } \\
\text { read }\end{array}$ & Notes & $\begin{array}{l}\text { Loca- } \\
\text { tion } \\
\text { code }\end{array}$ & $\begin{array}{c}\text { Site } \\
\text { nomenclature }\end{array}$ & $\begin{array}{l}\text { Amount } \\
\text { (Inches) }\end{array}$ & $\begin{array}{l}\text { Date } \\
\text { read }\end{array}$ & Notes & $\begin{array}{l}\text { Loca- } \\
\text { tion } \\
\text { code }\end{array}$ & $\begin{array}{c}\text { Site } \\
\text { nomenclature }\end{array}$ & $\begin{array}{l}\text { Amount } \\
\text { (inches) }\end{array}$ & $\begin{array}{l}\text { Date } \\
\text { read }\end{array}$ & Notes \\
\hline \multicolumn{15}{|c|}{ STORM OF JULY 12-13 \& 15, 1992-Continued } \\
\hline 23 & UE-25 UZN \#56 & 0 & $7 / 16$ & & 59 & USW UZ-N66 & 0 & $7 / 16$ & & 94 & HRF 4" Gage & $\mathrm{T}$ & $7 / 16$ & \\
\hline 24 & UE-25 UZN \#60 & 0 & $7 / 16$ & & 60 & USW UZ-N67 & 0 & $7 / 16$ & & 94 & HRF 8" Gage & $\mathrm{T}$ & $7 / 16$ & \\
\hline 25 & UE-25 UZN \#85 & 0 & $7 / 16$ & & 60 & N67 4" Gage & $\mathrm{T}$ & $7 / 16$ & & 95 & USW H-3 & 0 & $7 / 16$ & \\
\hline 26 & UE-25 UZN \#92 & .04 & $7 / 16$ & & 61 & USW UZ-N68 & 0 & $7 / 16$ & & 96 & Wea station & 0 & $7 / 16$ & \\
\hline 26 & N92 4" Gage & NI & & & 62 & USW UZ-N69 & 0 & $7 / 16$ & & 97 & Wea station & $\mathbf{M}$ & & \\
\hline 27 & UE-25 UZN \#97 & 0 & $7 / 16$ & & 63 & USW UZ-N70 & 0 & $7 / 16$ & & 98 & Evaporation & 0 & $7 / 16$ & \\
\hline 28 & UE-29 UZN \#91 & .17 & $7 / 16$ & & 63 & N70 4" Gage & 0 & $7 / 16$ & & 99 & Wea station & 0 & $7 / 16$ & \\
\hline 28 & N91 4" Gage & NI & & & 64 & USW UZ-N71 & 0 & $7 / 16$ & & 99 & Wea station & 0 & $7 / 16$ & \\
\hline 29 & USW UZ-N11 & .12 & $7 / 16$ & & 64 & N71 4" Gage & 0 & $7 / 16$ & & 100 & G-3 & 0 & $7 / 16$ & \\
\hline 29 & N11 4" Gage & .23 & $7 / 16$ & & 65 & USW UZ-N72 & 0 & $7 / 16$ & & 100 & G.3 8" Gage & NI & & \\
\hline 30 & USW UZ-N15 & .09 & $7 / 16$ & & 66 & USW UZ-N73 & 0 & $7 / 16$ & & 101 & Wea station & 0 & $7 / 16$ & \\
\hline 31 & USW UZ-N16 & .09 & $7 / 16$ & & 67 & USW UZ-N74 & 0 & $7 / 16$ & & 101 & Wea station & $\mathrm{NI}$ & & \\
\hline 32 & USW UZ-N17 & .11 & $7 / 16$ & & 68 & USW UZ-N75 & 0 & $7 / 16$ & & 102 & Wea station & NI & & \\
\hline 33 & USW UZ-N24 & .01 & $7 / 16$ & & 68 & N75 4" Gage & 0 & $7 / 16$ & & 103 & Wea station & .01 & $7 / 16$ & \\
\hline 33 & N24 4" Gage & NI & & & 69 & USW UZ-N76 & 0 & $7 / 16$ & & 103 & Wea station & $\mathrm{NI}$ & & \\
\hline 34 & USW UZ-N25 & 0 & $7 / 16$ & & 70 & USW UZ-N77 & 0 & $7 / 16$ & & 104 & Fran Ridge & 0 & $7 / 16$ & \\
\hline 35 & USW UZ-N26 & 0 & $7 / 16$ & & 70 & N77 4" Gage & Removed & & & 104 & Fran Ridge (8") & NI & & \\
\hline 36 & USW UZ-N27 & $\mathbf{M}^{*}$ & & & 71 & USW UZ-N78 & 0 & $7 / 16$ & & 105 & Plug Hill & 0 & $7 / 16$ & \\
\hline 36 & N27 8" Gage & .03 & $7 / 16$ & & 72 & USW UZ-N79 & .05 & $7 / 16$ & & 106 & 4JA (8") & 0 & $7 / 16$ & \\
\hline 37 & USW UZ-N35 & $\mathrm{NI}$ & & & 73 & USW UZ-N80 & 0 & $7 / 16$ & & 107 & Knothead Gap & $\mathrm{NI}$ & & \\
\hline 38 & USW UZ-N36 & 0 & $7 / 16$ & & 73 & N80 4" Gage & 0 & $7 / 16$ & & 108 & Upper Forty Mile & NI & & \\
\hline
\end{tabular}


Table 2. Summary of precipitation data from the nonrecording gage network at Yucca Mountain, Nevada, for water years 1992 and 1993 --Continued

\begin{tabular}{|c|c|c|c|c|c|c|c|c|c|c|c|c|c|c|}
\hline $\begin{array}{l}\text { Loce- } \\
\text { tion } \\
\text { code }\end{array}$ & $\begin{array}{c}\text { Site } \\
\text { nomenclature }\end{array}$ & $\begin{array}{l}\text { Amount } \\
\text { (inches) }\end{array}$ & $\begin{array}{l}\text { Date } \\
\text { read }\end{array}$ & Notes & $\begin{array}{l}\text { Loca- } \\
\text { tion } \\
\text { code }\end{array}$ & $\begin{array}{c}\text { Site } \\
\text { nomenclature }\end{array}$ & $\begin{array}{l}\text { Amount } \\
\text { (inches) }\end{array}$ & $\begin{array}{l}\text { Date } \\
\text { read }\end{array}$ & Notes & $\begin{array}{l}\text { Loca- } \\
\text { tion } \\
\text { code }\end{array}$ & $\begin{array}{c}\text { Site } \\
\text { nomenclature }\end{array}$ & $\begin{array}{l}\text { Amount } \\
\text { (inehes) }\end{array}$ & $\begin{array}{l}\text { Date } \\
\text { read }\end{array}$ & Notes \\
\hline \multicolumn{15}{|c|}{ STORM OF AUGUST 2,1992 } \\
\hline 1 & UE-25 UZN \#1 & .26 & $8 / 3$ & & 39 & USW UZ-N37 & .07 & $8 / 3$ & & 74 & USW UZ-N81 & 0 & $8 / 4$ & \\
\hline 2 & UE-25 UZN \#2 & .36 & $8 / 3$ & & 39 & N37 4" Gage & .10 & $8 / 3$ & & 75 & USW UZ-N82 & 0 & $8 / 4$ & \\
\hline 3 & UE-25 UZN \#3 & .35 & $8 / 3$ & & 40 & USW UZ-N40 & .03 & $8 / 4$ & & 75 & N82 4" Gage & & & Removed \\
\hline 4 & UE-25 UZN \#4 & .37 & $8 / 3$ & & 40 & N40 4" Gage & NI & & & 76 & USW UZ-N83 & 0 & $8 / 4$ & \\
\hline 4 & N4 4" Gage & NI & & & 41 & USW UZ-N41 & .01 & $8 / 4$ & & 77 & USW UZ-N84 & 0 & $8 / 4$ & \\
\hline 5 & UE-25 UZN \#5 & .37 & $8 / 3$ & & 42 & USW UZ-N42 & .02 & $8 / 4$ & & 78 & USW UZ-N86 & 0 & $8 / 4$ & \\
\hline 6 & UE-25 UZN \#6 & .37 & $8 / 3$ & & 43 & USW UZ-N43 & .01 & $8 / 4$ & & 79 & USW UZ-N87 & 0 & $8 / 4$ & \\
\hline 7 & UE-25 UZN \#7 & .40 & $8 / 3$ & & 43 & N43 4" Gage & NI & & & 80 & USW UZ-N88 & 0 & $8 / 4$ & \\
\hline 8 & UE-25 UZN \#8 & .43 & $8 / 3$ & & 44 & USW UZ-N44 & .01 & $8 / 4$ & & 81 & USW UZ-N89 & $\mathbf{0}$ & $8 / 4$ & \\
\hline 9 & UE-25 UZN \#9 & .39 & $8 / 3$ & & 45 & USW UZ-N45 & .02 & $8 / 4$ & & 82 & USW UZ-N90 & 0 & $8 / 4$ & \\
\hline 10 & UE-25 UZN \#10 & .32 & $8 / 3$ & & 46 & USW UZ-N46 & .10 & $8 / 4$ & & 82 & N90 4" Gage & 0 & $8 / 4$ & \\
\hline 10 & N10 4" Gage & NI & & & 47 & USW UZ-N47 & .08 & $8 / 4$ & & 83 & USW UZ-N93 & .03 & $8 / 4$ & \\
\hline 11 & UE-25 UZN \#12 & .36 & $8 / 3$ & & 48 & USW UZ-N48 & 0 & $8 / 4$ & & 84 & USW UZ-N94 & .02 & $8 / 4$ & \\
\hline 12 & UE-25 UZN \#13 & .18 & $8 / 3$ & & 49 & USW UZ-N49 & 0 & $8 / 4$ & & 85 & USW UZ-N95 & .03 & $8 / 4$ & \\
\hline 13 & UE-25 UZN \#14 & .15 & $8 / 3$ & & 50 & USW UZ-N50 & 0 & $8 / 4$ & & 86 & USW UZ-N96 & .05 & $8 / 4$ & \\
\hline 14 & UE-25 UZN \#18 & .13 & $8 / 4$ & & 50 & N50 4" Gage & $\mathrm{NI}$ & & & 87 & USW UZ-N98 & 0 & $8 / 4$ & \\
\hline 15 & UE-25 UZN \#19 & .01 & $8 / 4$ & & 51 & USW UZ-N51 & 0 & $8 / 4$ & & 87 & N98 4" Gage & NI & & \\
\hline 16 & UE-25 UZN \#20 & .01 & $8 / 4$ & & 52 & USW UZ-N52 & 0 & $8 / 4$ & & 88 & USW UZ-13 & 0 & $8 / 4$ & \\
\hline 17 & UE-25 UZN \#21 & 0 & $8 / 4$ & & 53 & USW UZ-N53 & 0 & $8 / 4$ & & 88 & UZ-13 4" Gage & $\mathbf{T}$ & $8 / 4$ & \\
\hline 17 & N21 4" Gage & NI & & & 54 & USW UZ-N54 & 0 & $8 / 4$ & & 89 & USW GA-1 & .02 & $8 / 4$ & \\
\hline 18 & UE-25 UZN \#22 & .01 & $8 / 4$ & & 54 & N54 4" Gage & 0 & $8 / 4$ & & 90 & USW G-2 & .03 & & \\
\hline 19 & UE-25 UZN \#23 & .01 & $8 / 4$ & & 55 & USW UZ-N55 & 0 & $8 / 4$ & & 91 & UE25 WT-4 & .21 & $8 / 4$ & \\
\hline 20 & UE-25 UZN \#28 & 0 & $8 / 3$ & & 56 & USW UZ-N57 & 0 & $8 / 4$ & & 92 & UE25 WT-18 & .51 & $8 / 4$ & \\
\hline 21 & UE-25 UZN \#29 & $\mathbf{0}$ & $8 / 3$ & & 57 & USW UZ-N64 & 0 & $8 / 4$ & & 93 & USW H-5 & 0 & $8 / 4$ & \\
\hline 22 & UE-25 UZN \#30 & 0 & $8 / 3$ & & 58 & USW UZ-N65 & 0 & $8 / 4$ & & 94 & HRF Wedge & 0 & $8 / 4$ & \\
\hline
\end{tabular}


Table 2. Summary of precipitation data from the nonrecording gage network at Yucca Mountain, Nevada, for water years 1992 and $1993--$ Continued

\begin{tabular}{|c|c|c|c|c|c|c|c|c|c|c|c|c|c|c|}
\hline $\begin{array}{l}\text { Loca- } \\
\text { tion } \\
\text { code }\end{array}$ & $\begin{array}{c}\text { Site } \\
\text { nomenclature }\end{array}$ & $\begin{array}{l}\text { Amount } \\
\text { (inches) }\end{array}$ & $\begin{array}{l}\text { Date } \\
\text { read }\end{array}$ & Notes & $\begin{array}{l}\text { Loca- } \\
\text { tlon } \\
\text { code }\end{array}$ & $\begin{array}{c}\text { Site } \\
\text { nomenclature }\end{array}$ & $\begin{array}{l}\text { Amount } \\
\text { (inches) }\end{array}$ & $\begin{array}{l}\text { Date } \\
\text { read }\end{array}$ & Notes & $\begin{array}{l}\text { Loca- } \\
\text { tlon } \\
\text { code }\end{array}$ & $\begin{array}{c}\text { Site } \\
\text { nomenclature }\end{array}$ & $\begin{array}{l}\text { Amount } \\
\text { (inches) }\end{array}$ & $\begin{array}{l}\text { Date } \\
\text { read }\end{array}$ & Notes \\
\hline \multicolumn{15}{|c|}{ STORM OF AUGUST 2, 1992-Continued } \\
\hline 23 & UE-25 UZN \#56 & 0 & $8 / 3$ & & 59 & USW UZ-N66 & 0 & $8 / 4$ & & 94 & HRF 4" Gage & 0 & $8 / 4$ & \\
\hline 24 & UE-25 UZN \#60 & 0 & $8 / 3$ & & 60 & USW UZ-N67 & 0 & $8 / 4$ & & 94 & HRF 8" Gage & 0 & $8 / 4$ & \\
\hline 25 & UE-25 UZN \#85 & 0 & $8 / 3$ & & 60 & N67 4" Gage & $\mathrm{T}$ & $8 / 4$ & & 95 & USW H-3 & 0 & $8 / 4$ & \\
\hline 26 & UE-25 UZN \#92 & .01 & $8 / 3$ & & 61 & USW UZ-N68 & 0 & $8 / 4$ & & 96 & Wea station & .01 & $8 / 4$ & \\
\hline 26 & N92 4" Gage & $\mathrm{NI}$ & & & 62 & USW UZ-N69 & 0 & $8 / 4$ & & 97 & Wea station & $\mathbf{M}$ & & \\
\hline 27 & UE-25 UZN \#97 & 0 & $8 / 3$ & & 63 & USW UZ-N70 & .05 & $8 / 4$ & & 98 & Evaporation & 0 & $8 / 4$ & \\
\hline 28 & UE-29 UZN \#91 & .12 & $8 / 3$ & & 63 & N70 4" Gage & .12 & $8 / 4$ & & 99 & Wea station & .02 & $8 / 4$ & \\
\hline 28 & N91 4" Gage & NI & & & 64 & USW UZ-N71 & .01 & $8 / 4$ & & 99 & Wea station & .03 & $8 / 4$ & \\
\hline 29 & USW UZ-N1I & 0 & $8 / 3$ & & 64 & N71 4" Gage & .03 & $8 / 4$ & & 100 & G-3 & 0 & $8 / 4$ & \\
\hline 29 & N11 4" Gage & .02 & $8 / 3$ & & 65 & USW UZ-N72 & $\mathbf{M}^{*}$ & & & 100 & G-3 8" Gage & 0 & $8 / 4$ & \\
\hline 30 & USW UZ-N15 & .08 & $8 / 3$ & & 66 & USW UZ-N73 & $\mathrm{M}^{*}$ & & & 101 & Wea station & 0 & $8 / 4$ & \\
\hline 31 & USW UZ-N16 & .07 & $8 / 3$ & & 67 & USW UZ-N74 & .02 & $8 / 4$ & & 101 & Wea station & 0 & $8 / 4$ & \\
\hline 32 & USW UZ-N17 & .03 & $8 / 3$ & & 68 & USW UZ-N75 & $\mathbf{M}^{*}$ & & & 102 & Wea station & 0 & $8 / 4$ & \\
\hline 33 & USW UZ-N24 & .05 & $8 / 3$ & & 68 & N75 4" Gage & $\mathbf{M}^{*}$ & & & 103 & Wea station & .08 & $8 / 4$ & \\
\hline 33 & N24 4" Gage & NI & & & 69 & USW UZ-N76 & $\mathrm{M}^{*}$ & & & 103 & Wea station & .12 & $8 / 4$ & \\
\hline 34 & USW UZ-N25 & .05 & $8 / 3$ & & 70 & USW UZ-N77 & 0 & $8 / 4$ & & 104 & Fran Ridge & 0 & $8 / 4$ & \\
\hline 35 & USW UZ-N26 & .04 & $8 / 3$ & & 70 & N77 4" Gage & Removed & & & 104 & Fran Ridge (8") & 0 & $8 / 4$ & \\
\hline 36 & USW UZ-N27 & .05 & $8 / 4$ & $* *$ & 71 & USW UZ-N78 & 0 & $8 / 4$ & & 105 & Plug Hill & 0 & $8 / 4$ & \\
\hline 36 & N27 8" Gage & .08 & $8 / 4$ & & 72 & USW UZ-N79 & 0 & & & 106 & 4JA (8") & .01 & $8 / 4$ & \\
\hline 37 & USW UZ-N35 & NI & & & 73 & USW UZ-N80 & 0 & $8 / 4$ & & 107 & Knothead Gap & $\mathrm{NI}$ & & \\
\hline 38 & USW UZ-N36 & .37 & $8 / 3$ & & 73 & N80 4" Gage & 0 & $8 / 4$ & & 108 & Upper Forty Mile & NI & & \\
\hline
\end{tabular}


Table 2. Summary of precipitation data from the nonrecording gage network at Yucca Mountain, Nevada, for water years 1992 and 1993 --Continued

\begin{tabular}{|c|c|c|c|c|c|c|c|c|c|c|c|c|c|c|}
\hline $\begin{array}{l}\text { Loca- } \\
\text { tion } \\
\text { code }\end{array}$ & $\begin{array}{c}\text { Site } \\
\text { nomenclature }\end{array}$ & $\begin{array}{l}\text { Amount } \\
\text { (inches) }\end{array}$ & $\begin{array}{l}\text { Date } \\
\text { read }\end{array}$ & Notes & $\begin{array}{l}\text { Loca- } \\
\text { tlon } \\
\text { code }\end{array}$ & $\begin{array}{c}\text { Site } \\
\text { nomenclature }\end{array}$ & $\begin{array}{l}\text { Amount } \\
\text { (inches) }\end{array}$ & $\begin{array}{l}\text { Date } \\
\text { read }\end{array}$ & Notes & $\begin{array}{l}\text { Loca- } \\
\text { tion } \\
\text { code }\end{array}$ & $\begin{array}{c}\text { Site } \\
\text { nomenclature }\end{array}$ & $\begin{array}{l}\text { Amount } \\
\text { (Inches) }\end{array}$ & $\begin{array}{l}\text { Date } \\
\text { read }\end{array}$ & Notes \\
\hline \multicolumn{15}{|c|}{ STORM OF AUGUST 11, 1992} \\
\hline 1 & UE-25 UZN \#1 & 0 & $8 / 13$ & & 39 & USW UZ-N37 & .06 & $8 / 13$ & & 74 & USW UZ-N81 & .64 & $8 / 14$ & \\
\hline 2 & UE-25 UZN \#2 & 0 & $8 / 13$ & & 39 & N37 4" Gage & .07 & $8 / 13$ & & 75 & USW UZ-N82 & .63 & $8 / 14$ & \\
\hline 3 & UE-25 UZN \#3 & 0 & $8 / 13$ & & 40 & USW UZ-N40 & .02 & $8 / 13$ & & 75 & N82 4" Gage & & & Removed \\
\hline 4 & UE-25 UZN \#4 & 0 & $8 / 13$ & & 40 & N40 4" Gage & NI & & & 76 & USW UZ-N83 & .41 & $8 / 14$ & \\
\hline 4 & N4 4" Gage & NI & & & 41 & USW UZ-N41 & .07 & $8 / 13$ & & 77 & USW UZ-N84 & .29 & $8 / 14$ & \\
\hline 5 & UE-25 UZN \#5 & 0 & $8 / 13$ & & 42 & USW UZ-N42 & .10 & $8 / 13$ & & 78 & USW UZ-N86 & .39 & $8 / 14$ & \\
\hline 6 & UE-25 UZN \#6 & 0 & $8 / 13$ & & 43 & USW UZ-N43 & .07 & $8 / 13$ & & 79 & USW UZ-N87 & .28 & $8 / 14$ & \\
\hline 7 & UE-25 UZN \#7 & 0 & $8 / 13$ & & 43 & N43 4" Gage & $\mathrm{NI}$ & & & 80 & USW UZ-N88 & .37 & $8 / 14$ & \\
\hline 8 & UE-25 UZN \#8 & 0 & $8 / 13$ & & 44 & USW UZ-N44 & .09 & $8 / 13$ & & 81 & USW UZ-N89 & .30 & $8 / 14$ & \\
\hline 9 & UE-25 UZN \#9 & 0 & $8 / 13$ & & 45 & USW UZ-N45 & .09 & $8 / 13$ & & 82 & USW UZ-N90 & .31 & $8 / 14$ & \\
\hline 10 & UE-25 UZN \#10 & 0 & $8 / 13$ & & 46 & USW UZ-N46 & .01 & $8 / 13$ & & 82 & N90 4" Gage & .35 & $8 / 14$ & \\
\hline 10 & N10 4" Gage & $\mathrm{NI}$ & & & 47 & USW UZ-N47 & 0 & $8 / 13$ & & 83 & USW UZ-N93 & .51 & $8 / 12$ & \\
\hline 11 & UE-25 UZN \#12 & 0 & $8 / 13$ & & 48 & USW UZ-N48 & .34 & $8 / 13$ & & 84 & USW UZ-N94 & .51 & $8 / 12$ & \\
\hline 12 & UE-25 UZN \#13 & 0 & $8 / 13$ & & 49 & USW UZ-N49 & .37 & $8 / 13$ & & 85 & USW UZ-N95 & .36 & $8 / 12$ & \\
\hline 13 & UE-25 UZN \#14 & 0 & $8 / 13$ & & 50 & USW UZ-N50 & .35 & $8 / 13$ & & 86 & USW UZ-N96 & .45 & $8 / 12$ & \\
\hline 14 & UE-25 UZN \#18 & .01 & $8 / 13$ & & 50 & N50 4" Gage & NI & & & 87 & USW UZ-N98 & .17 & $8 / 12$ & \\
\hline 15 & UE-25 UZN \#19 & .11 & $8 / 13$ & & 51 & USW UZ-N51 & .32 & $8 / 13$ & & 87 & N98 4" Gage & $\mathrm{NI}$ & & \\
\hline 16 & UE-25 UZN \#20 & .11 & $8 / 13$ & & 52 & USW UZ-N52 & .29 & $8 / 13$ & & 88 & USW UZ 13 & .42 & $8 / 12$ & \\
\hline 17 & UE-25 UZN \#21 & .08 & $8 / 13$ & & 53 & USW UZ-N53 & $\mathbf{M}$ & $8 / 13$ & & 88 & UZ-13 4" Gage & .37 & $8 / 12$ & \\
\hline 17 & N21 4" Gage & NI & & & 54 & USW UZ-N54 & .27 & $8 / 13$ & & 89 & USW GA-1 & .01 & $8 / 13$ & \\
\hline 18 & UE-25 UZN \#22 & .09 & $8 / 13$ & & 54 & N54 4" Gage & .28 & $8 / 13$ & & 90 & USW G-2 & 0 & $8 / 13$ & \\
\hline 19 & UE-25 UZN \#23 & .09 & $8 / 13$ & & 55 & USW UZ-N55 & .27 & $8 / 13$ & & 91 & UE25 WT-4 & 0 & $8 / 13$ & \\
\hline 20 & UE-25 UZN \#28 & .07 & $8 / 13$ & & 56 & USW UZ-N57 & .51 & $8 / 13$ & & 92 & UE25 WT-18 & 0 & $8 / 13$ & \\
\hline 21 & UE-25 UZN \#29 & .09 & $8 / 13$ & & 57 & USW UZ-N64 & .22 & $8 / 13$ & & 93 & USW H-5 & .12 & $8 / 13$ & \\
\hline 22 & UE-25 UZN \#30 & .09 & $8 / 13$ & & 58 & USW UZ-N65 & .29 & $8 / 13$ & & 94 & HRF Wedge & .01 & $8 / 13$ & \\
\hline
\end{tabular}


Table 2. Summary of precipitation data from the nonrecording gage network at Yucca Mountain, Nevada, for water years 1992 and 1993--Continued

\begin{tabular}{|c|c|c|c|c|c|c|c|c|c|c|c|c|c|c|}
\hline $\begin{array}{l}\text { Loca- } \\
\text { tion } \\
\text { code }\end{array}$ & $\begin{array}{c}\text { Site } \\
\text { nomenclature }\end{array}$ & $\begin{array}{l}\text { Amount } \\
\text { (inches) }\end{array}$ & $\begin{array}{l}\text { Date } \\
\text { read }\end{array}$ & Notes & $\begin{array}{l}\text { Loca- } \\
\text { tion } \\
\text { code }\end{array}$ & $\begin{array}{c}\text { Slte } \\
\text { nomenclature }\end{array}$ & $\begin{array}{l}\text { Amount } \\
\text { (inches) }\end{array}$ & $\begin{array}{l}\text { Date } \\
\text { read }\end{array}$ & Notes & $\begin{array}{l}\text { Loca- } \\
\text { tion } \\
\text { code }\end{array}$ & $\begin{array}{c}\text { Site } \\
\text { nomenclature }\end{array}$ & $\begin{array}{l}\text { Amount } \\
\text { (inches) }\end{array}$ & $\begin{array}{l}\text { Date } \\
\text { read }\end{array}$ & Notes \\
\hline \multicolumn{15}{|c|}{ STORM OF AUGUST 11, 1992-Continued } \\
\hline 23 & UE-25 UZN \#56 & .16 & $8 / 13$ & & 59 & USW UZ-N66 & .33 & $8 / 13$ & & 94 & HRF 4" Gage & .01 & $8 / 13$ & \\
\hline 24 & UE-25 UZN \#60 & .09 & $8 / 13$ & & 60 & USW UZ-N67 & .63 & $8 / 13$ & & 94 & HRFF 8" Gage & 0 & $8 / 13$ & \\
\hline 25 & UE-25 UZN \#85 & .16 & $8 / 13$ & & 60 & N67 4" Gage & .67 & $8 / 13$ & & 95 & USW H-3 & .36 & $8 / 13$ & \\
\hline 26 & UE-25 UZN \#92 & 0 & $8 / 13$ & & 61 & USW UZ-N68 & .61 & $8 / 13$ & & 96 & Wea station & .36 & $8 / 13$ & \\
\hline 26 & N92 4" Gage & NI & & & 62 & USW UZ-N69 & .51 & $8 / 13$ & & 97 & Wea station & M & & \\
\hline 27 & UE-25 UZN \#97 & .07 & $8 / 13$ & & 63 & USW UZ-N70 & 0 & $8 / 13$ & & 98 & Evaporation & 0 & $8 / 12$ & \\
\hline 28 & UE-29 UZN \#91 & 0 & $8 / 13$ & & 63 & N70 4" Gage & 0 & $8 / 13$ & & 99 & Wea station & .49 & $8 / 12$ & \\
\hline 28 & N91 4" Gage & NI & & & 64 & USW UZ-N71 & .30 & $8 / 13$ & & 99 & Wea station & .49 & $8 / 12$ & \\
\hline 29 & USW UZ-N11 & 0 & $8 / 13$ & & 64 & N71 4" Gage & .35 & $8 / 13$ & & 100 & G-3 & .45 & $8 / 12$ & \\
\hline 29 & N11 4" Gage & 0 & $8 / 13$ & & 65 & USW UZ-N72 & .38 & $8 / 13$ & ** & 100 & G-3 8" Gage & .48 & $8 / 12$ & \\
\hline 30 & USW UZ-N15 & .08 & $8 / 13$ & & 66 & USW UZ-N73 & .37 & $8 / 13$ & $* *$ & 101 & Wea station & .07 & $8 / 12$ & \\
\hline 31 & USW UZ-N16 & 0 & $8 / 13$ & & 67 & USW UZ-N74 & .35 & $8 / 13$ & & 101 & Wea station & .07 & $8 / 12$ & \\
\hline 32 & USW UZ-N17 & .03 & $8 / 13$ & & 68 & USW UZ-N75 & .37 & $8 / 13$ & *** & 102 & Wea station & 0 & $8 / 12$ & \\
\hline 33 & USW UZ-N24 & .18 & $8 / 13$ & & 68 & N75 4" Gage & .41 & $8 / 13$ & $* *$ & 103 & Wea station & .01 & $8 / 12$ & \\
\hline 33 & N24 4" Gage & NI & & & 69 & USW UZ-N76 & .42 & $8 / 13$ & $* *$ & 103 & Wea station & .03 & $8 / 12$ & \\
\hline 34 & USW UZ-N25 & .15 & $8 / 13$ & & 70 & USW UZ-N77 & .75 & $8 / 14$ & & 104 & Fran Ridge & 0 & $8 / 12$ & \\
\hline 35 & USW UZ-N26 & .12 & $8 / 13$ & & 70 & N77 4" Gage & Removed & & & 104 & Fran Ridge (8") & 0 & $8 / 12$ & \\
\hline 36 & USW UZ-N27 & .02 & $8 / 13$ & & 71 & USW UZ-N78 & .55 & $8 / 14$ & & 105 & Plug Hill & .41 & $8 / 12$ & \\
\hline 36 & N27 8" Gage & .03 & $8 / 13$ & & 72 & USW UZ-N79 & .55 & $8 / 14$ & & 106 & 4JA (8") & 0 & $8 / 12$ & \\
\hline 37 & USW UZ-N35 & NI & & & 73 & USW UZ-N80 & .57 & $8 / 14$ & & 107 & Knothead Gap & NI & & \\
\hline 38 & USW UZ-N36 & 0 & $8 / 13$ & & 73 & N80 4" Gage & .61 & $8 / 14$ & & 108 & Upper Forty Mile & NI & & \\
\hline
\end{tabular}


Table 2. Summary of precipitation data from the nonrecording gage network at Yucca Mountain, Nevada, for water years 1992 and 1993--Continued

\begin{tabular}{|c|c|c|c|c|c|c|c|c|c|c|c|c|c|c|}
\hline $\begin{array}{l}\text { Loca- } \\
\text { tion } \\
\text { code }\end{array}$ & $\begin{array}{c}\text { Site } \\
\text { nomenclature }\end{array}$ & $\begin{array}{l}\text { Amount } \\
\text { (inches) }\end{array}$ & $\begin{array}{l}\text { Date } \\
\text { read }\end{array}$ & Notes & $\begin{array}{l}\text { Loca- } \\
\text { tion } \\
\text { code }\end{array}$ & $\begin{array}{c}\text { Site } \\
\text { nomenclature }\end{array}$ & $\begin{array}{l}\text { Amount } \\
\text { (inches) }\end{array}$ & $\begin{array}{l}\text { Date } \\
\text { read }\end{array}$ & Notes & $\begin{array}{l}\text { Loca- } \\
\text { tion } \\
\text { code }\end{array}$ & $\begin{array}{c}\text { Site } \\
\text { nomenclature }\end{array}$ & $\begin{array}{l}\text { Amount } \\
\text { (inches) }\end{array}$ & $\begin{array}{l}\text { Date } \\
\text { read }\end{array}$ & Notes \\
\hline \multicolumn{15}{|c|}{ STORM OF OCTOBER 24, 1992} \\
\hline 1 & UE-25 UZN \#1 & .30 & $10 / 27$ & & 39 & USW UZ-N37 & .22 & $10 / 27$ & & 74 & USW UZ-N81 & .10 & $10 / 27$ & \\
\hline 2 & UE-25 UZN \#2 & .33 & $10 / 27$ & & 39 & N37 4" Gage & .33 & $10 / 27$ & & 75 & USW UZ-N82 & .11 & $10 / 27$ & \\
\hline 3 & UE-25 UZN \#3 & .28 & $10 / 27$ & & 40 & USW UZ-N40 & .25 & $10 / 27$ & & 75 & N82 4" Gage & & & Removed \\
\hline 4 & UE-25 UZN \#4 & .30 & $10 / 27$ & & 40 & N40 4" Gage & NI & & & 76 & USW UZ-N83 & .12 & $10 / 27$ & \\
\hline 4 & N4 4" Gage & NI & & & 41 & USW UZ-N4I & .23 & $10 / 27$ & & 77 & USW UZ-N84 & .13 & $10 / 27$ & \\
\hline 5 & UE-25 UZN \#5 & .29 & $10 / 27$ & & 42 & USW UZ-N42 & .22 & $10 / 27$ & & 78 & USW UZ-N86 & .15 & $10 / 27$ & \\
\hline 6 & UE-25 UZN \#6 & .32 & $10 / 27$ & & 43 & USW UZ-N43 & .24 & $10 / 27$ & & 79 & USW UZ-N87 & .13 & $10 / 27$ & \\
\hline 7 & UE-25 UZN \#7 & .30 & $10 / 27$ & & 43 & N43 4" Gage & NI & & & 80 & USW UZ-N88 & .11 & $10 / 27$ & \\
\hline 8 & UE-25 UZN \#8 & .29 & $10 / 27$ & & 44 & USW UZ-N44 & .25 & $10 / 27$ & & 81 & USW UZ-N89 & .07 & $10 / 27$ & \\
\hline 9 & UE-25 UZN \#9 & .29 & $10 / 27$ & & 45 & USW UZ-N45 & .25 & $10 / 27$ & & 82 & USW UZ-N90 & .13 & $10 / 27$ & \\
\hline 10 & UE-25 UZN \#10 & .30 & $10 / 27$ & & 46 & USW UZ-N46 & .30 & $10 / 27$ & & 82 & N90 4" Gage & .35 & $10 / 27$ & \\
\hline 10 & N10 4" Gage & NI & & & 47 & USW UZ-N47 & .25 & $10 / 27$ & & 83 & USW UZ-N93 & .13 & $10 / 27$ & \\
\hline 11 & UE-25 UZN \#12 & .32 & $10 / 27$ & & 48 & USW UZ-N48 & .21 & $10 / 27$ & & 84 & USW UZ-N94 & .15 & $10 / 27$ & \\
\hline 12 & UE-25 UZN \#13 & .35 & $10 / 27$ & & 49 & USW UZ-N49 & .20 & $10 / 27$ & & 85 & USW UZ-N95 & .15 & $10 / 27$ & \\
\hline 13 & UE-25 UZN \#14 & .30 & $10 / 27$ & & 50 & USW UZ-N50 & .22 & $10 / 27$ & & 86 & USW UZ-N96 & .18 & $10 / 27$ & \\
\hline 14 & UE-25 UZN \#18 & .25 & $10 / 27$ & & 50 & N50 4" Gage & NI & & & 87 & USW UZ-N98 & .25 & $10 / 27$ & \\
\hline 15 & UE-25 UZN \#19 & .24 & $10 / 27$ & & 51 & USW UZ-N51 & .21 & $10 / 27$ & & 87 & N98 4" Gage & $\mathrm{NI}$ & & \\
\hline 16 & UE-25 UZN \#20 & .25 & $10 / 27$ & & 52 & USW UZ-N52 & .19 & $10 / 27$ & & 88 & USW UZ 13 & .13 & $10 / 27$ & \\
\hline 17 & UE-25 UZN \#21 & .23 & $10 / 27$ & & 53 & USW UZ-N53 & .21 & $10 / 27$ & & 88 & UZ-13 4" Gage & .16 & $10 / 27$ & \\
\hline 17 & N21 4" Gage & NI & & & 54 & USW UZ-N54 & .20 & $10 / 27$ & & 89 & USW GA-I & .39 & $10 / 27$ & \\
\hline 18 & UE-25 UZN \#22 & .23 & $10 / 27$ & & 54 & N54 4" Gage & .24 & $10 / 27$ & & 90 & USW G-2 & .39 & $10 / 27$ & \\
\hline 19 & UE-25 UZN \#23 & .23 & $10 / 27$ & & 55 & USW UZ-N55 & .28 & $10 / 27$ & & 91 & UE25 WT-4 & .29 & $10 / 27$ & \\
\hline 20 & UE-25 UZN \#28 & .25 & $10 / 27$ & & 56 & USW UZ-N57 & .13 & $10 / 27$ & & 92 & UE25 WT-18 & .30 & $10 / 27$ & \\
\hline 21 & UE-25 UZN \#29 & .24 & $10 / 27$ & & 57 & USW UZ-N64 & .46 & $10 / 27$ & & 93 & USW H-5 & .18 & $10 / 27$ & \\
\hline 22 & UE-25 UZN \#30 & .25 & $10 / 27$ & & 58 & USW UZ-N65 & .18 & $10 / 27$ & & 94 & HRF Wedge & .36 & $10 / 27$ & \\
\hline
\end{tabular}




\begin{tabular}{|c|c|c|c|c|c|c|c|c|c|c|c|c|c|c|}
\hline $\begin{array}{l}\text { Loca- } \\
\text { tion } \\
\text { code }\end{array}$ & $\begin{array}{c}\text { Site } \\
\text { nomenclature }\end{array}$ & $\begin{array}{l}\text { Amount } \\
\text { (inches) }\end{array}$ & $\begin{array}{l}\text { Date } \\
\text { read }\end{array}$ & Notes & $\begin{array}{l}\text { Loca- } \\
\text { tion } \\
\text { code }\end{array}$ & $\begin{array}{c}\text { Site } \\
\text { nomenelature }\end{array}$ & $\begin{array}{l}\text { Amount } \\
\text { (inches) }\end{array}$ & $\begin{array}{l}\text { Date } \\
\text { read }\end{array}$ & Notes & $\begin{array}{l}\text { Loca- } \\
\text { tion } \\
\text { code }\end{array}$ & $\begin{array}{c}\text { Site } \\
\text { nomenclature }\end{array}$ & $\begin{array}{l}\text { Amount } \\
\text { (inches) }\end{array}$ & $\begin{array}{l}\text { Date } \\
\text { read }\end{array}$ & Notes \\
\hline \multicolumn{15}{|c|}{ STORM OF OCTOBER 24, 1992-Conthued } \\
\hline 23 & UE-25 UZN \#56 & .23 & $10 / 27$ & & 59 & USW UZ-N66 & .16 & $10 / 27$ & & 94 & HRF 4" Gage & .42 & $10 / 27$ & \\
\hline 24 & UE-25 UZN \#60 & .24 & $10 / 27$ & & 60 & USW UZ-NG7 & .17 & $10 / 27$ & & 94 & HRF 8" Gage & .43 & $10 / 27$ & \\
\hline 25 & UE-25 UZN \#85 & .21 & $10 / 27$ & & 60 & N67 4" Gage & .22 & $10 / 27$ & & 95 & USW H-3 & .07 & $10 / 27$ & \\
\hline 26 & UE-25 UZN \#92 & .10 & $10 / 27$ & & 61 & USW UZ-N68 & .19 & $10 / 27$ & & 96 & Wea station & .12 & $10 / 27$ & \\
\hline 26 & N92 4" Gage & NI & & & 62 & USW UZ-N69 & .17 & $10 / 27$ & & 97 & Wea station & M & & \\
\hline 27 & UE-25 UZN \#97 & .24 & $10 / 27$ & & 63 & USW UZ-N70 & .41 & $10 / 27$ & & 98 & Evaporation & .42 & $10 / 27$ & \\
\hline 28 & UE-29 UZN \#91 & .18 & $10 / 27$ & & 63 & N70 4" Gage & .43 & $10 / 27$ & & 99 & Wea station & .17 & $10 / 27$ & \\
\hline 28 & N91 4" Gage & NI & & & 64 & USW UZ-N71 & .12 & $10 / 27$ & & 99 & Wea station & .21 & $10 / 27$ & \\
\hline 29 & USW UZ-N11 & .35 & $10 / 27$ & & 64 & N71 4" Gage & .14 & $10 / 27$ & & 100 & G-3 & .13 & $10 / 27$ & \\
\hline 29 & N11 4" Gage & .39 & $10 / 27$ & & 65 & USW UZ-N72 & .13 & $10 / 27$ & & 100 & G-3 8" Gage & .16 & $10 / 27$ & \\
\hline 30 & USW UZ-N15 & .41 & $10 / 27$ & & 66 & USW UZ-N73 & .19 & $10 / 27$ & & 101 & Wea station & .35 & $10 / 27$ & \\
\hline 31 & USW UZ-N16 & .40 & $10 / 27$ & & 67 & USW UZ-N74 & .13 & $10 / 27$ & & 101 & Wea station & .34 & $10 / 27$ & \\
\hline 32 & USW UZ-N17 & .40 & $10 / 27$ & & 68 & USW UZ-N75 & .15 & $10 / 27$ & & 102 & Wea station & .45 & $10 / 27$ & \\
\hline 33 & USW UZ-N24 & .24 & $10 / 27$ & & 68 & N75 4" Gage & .21 & $10 / 27$ & & 103 & Wea station & .25 & $10 / 27$ & \\
\hline 33 & N24 4" Gage & $\mathrm{NI}$ & & & 69 & USW UZ-N76 & .15 & $10 / 27$ & & 103 & Wea station & .30 & $10 / 27$ & \\
\hline 34 & USW UZ-N25 & .24 & $10 / 27$ & & 70 & USW UZ-N77 & .11 & $10 / 27$ & & 104 & Fran Ridge & .26 & $10 / 27$ & \\
\hline 35 & USW UZ-N26 & .24 & $10 / 27$ & & 70 & N77 4" Gage & Removed & & & 104 & Fran Ridge (8") & .31 & $10 / 27$ & \\
\hline 36 & USW UZ-N27 & .22 & $10 / 27$ & & 71 & USW UZ-N78 & .12 & $10 / 27$ & & 105 & Plug Hill & .05 & $10 / 27$ & \\
\hline 36 & N27 8" Gage & .27 & $10 / 27$ & & 72 & USW UZ-N79 & .12 & $10 / 27$ & & 106 & 4JA (8") & .44 & $10 / 27$ & \\
\hline 37 & USW UZ-N35 & NI & & & 73 & USW UZ-N80 & .12 & $10 / 27$ & & 107 & Knothead Gap & NI & & \\
\hline 38 & USW UZ-N36 & .37 & $10 / 27$ & & 73 & N80 4" Gage & .16 & $10 / 27$ & & 108 & Upper Forty Mile & NI & & \\
\hline
\end{tabular}


Table 2. Summary of precipitation data from the nonrecording gage network at Yucca Mountain, Nevada, for water years 1992 and 1993--Continued

\begin{tabular}{|c|c|c|c|c|c|c|c|c|c|c|c|c|c|c|}
\hline $\begin{array}{l}\text { Loca- } \\
\text { tion } \\
\text { code }\end{array}$ & $\begin{array}{c}\text { Site } \\
\text { nomenclature }\end{array}$ & $\begin{array}{l}\text { Amount } \\
\text { (inches) }\end{array}$ & $\begin{array}{l}\text { Date } \\
\text { read }\end{array}$ & Notes & $\begin{array}{l}\text { Loca- } \\
\text { tion } \\
\text { code }\end{array}$ & $\begin{array}{c}\text { Site } \\
\text { nomenclature }\end{array}$ & $\begin{array}{l}\text { Amount } \\
\text { (inches) }\end{array}$ & $\begin{array}{l}\text { Date } \\
\text { read }\end{array}$ & Notes & $\begin{array}{l}\text { Loce- } \\
\text { tion } \\
\text { code }\end{array}$ & $\begin{array}{c}\text { Site } \\
\text { nomenclature }\end{array}$ & $\begin{array}{l}\text { Amount } \\
\text { (Inches) }\end{array}$ & $\begin{array}{l}\text { Date } \\
\text { read }\end{array}$ & Notes \\
\hline \multicolumn{15}{|c|}{ STORM OF OCTOBER 28, 1992} \\
\hline 1 & UE-25 UZN \#1 & .26 & $10 / 29$ & & 39 & USW UZ-N37 & .24 & $10 / 29$ & & 74 & USW UZ-N81 & $\mathbf{M}^{*}$ & & \\
\hline 2 & UE-25 UZN \#2 & .29 & $10 / 29$ & & 39 & N37 4" Gage & .27 & $10 / 29$ & & 75 & USW UZ-N82 & $\mathbf{M}^{*}$ & & \\
\hline 3 & UE-25 UZN \#3 & .23 & $10 / 29$ & & 40 & USW UZ-N40 & .22 & $10 / 29$ & & 75 & N82 4" Gage & & & Removed \\
\hline 4 & UE-25 UZN \#4 & .23 & $10 / 29$ & & 40 & N40 4" Gage & NI & & & 76 & USW UZ-N83 & $\mathrm{M}^{*}$ & & \\
\hline 4 & N4 4" Gage & NI & & & 41 & USW UZ-N41 & .22 & $10 / 29$ & & 77 & USW UZ-N84 & $\mathbf{M}^{*}$ & & \\
\hline 5 & UE-25 UZN \#5 & .22 & $10 / 29$ & & 42 & USW UZ-N42 & .23 & $10 / 29$ & & 78 & USW UZ-N86 & $\mathbf{M}^{*}$ & & \\
\hline 6 & UE-25 UZN \#6 & .25 & $10 / 29$ & & 43 & USW UZ-N43 & .23 & $10 / 29$ & & 79 & USW UZ-N87 & $\mathbf{M}^{*}$ & & \\
\hline 7 & UE-25 UZN \#7 & .22 & $10 / 29$ & & 43 & N43 4" Gage & NI & & & 80 & USW UZ-N88 & $\mathrm{M}^{*}$ & & \\
\hline 8 & UE-25 UZN \#8 & .22 & $10 / 29$ & & 44 & USW UZ-N44 & .22 & $10 / 29$ & & 81 & USW UZ-N89 & $\mathbf{M}^{*}$ & & \\
\hline 9 & UE-25 UZN \#9 & .26 & $10 / 29$ & & 45 & USW UZ-N4S & .25 & $10 / 29$ & & 82 & USW UZ-N9o & $\mathbf{M}^{*}$ & & \\
\hline 10 & UE-25 UZN \#10 & .26 & $10 / 29$ & & 46 & USW UZ-N46 & .31 & $10 / 29$ & & 82 & N90 4" Gage & $\mathbf{M}^{*}$ & & \\
\hline 10 & N10 4" Gage & $\mathrm{NI}$ & & & 47 & USW UZ-N47 & .26 & $10 / 29$ & & 83 & USW UZ-N93 & $\mathbf{M}^{*}$ & & \\
\hline 11 & UE-25 UZN \#12 & .25 & $10 / 29$ & & 48 & USW UZ-N48 & .25 & $10 / 29$ & & 84 & USW UZ-N94 & $\mathbf{M}^{*}$ & & \\
\hline 12 & UE-25 UZN \#13 & .30 & $10 / 29$ & & 49 & USW UZ-N49 & .25 & $10 / 29$ & & 85 & USW UZ-N9S & $\mathrm{M}^{*}$ & & \\
\hline 13 & UE-25 UZN \#14 & .25 & $10 / 29$ & & 50 & USW UZ-N50 & .25 & $10 / 29$ & & 86 & USW UZ-N96 & $\mathbf{M}^{*}$ & & \\
\hline 14 & UE-25 UZN \#1 8 & .24 & $10 / 29$ & & 50 & N50 4" Gage & NI & & & 87 & USW UZ-N98 & .25 & $10 / 29$ & \\
\hline 15 & UE-25 UZN \#19 & .25 & $10 / 29$ & & 51 & USW UZ-N51 & .26 & $10 / 29$ & & 87 & N98 4" Gage & NI & & \\
\hline 16 & UE-25 UZN \#20 & .25 & $10 / 29$ & & 52 & USW UZ-N52 & .26 & $10 / 29$ & & 88 & USW UZ-13 & $\mathbf{M}^{*}$ & & \\
\hline 17 & UE-25 UZN \#21 & .22 & $10 / 29$ & & 53 & USW UZ-N53 & .22 & $10 / 29$ & & 88 & UZ-13 4" Gage & $\mathbf{M}^{*}$ & & \\
\hline 17 & N21 4" Gage & NI & & & 54 & USW UZ-N54 & .22 & $10 / 29$ & & 89 & USW GA-1 & .32 & $10 / 29$ & \\
\hline 18 & UE-25 UZN \#22 & .23 & $10 / 29$ & & 54 & N54 4" Gage & .25 & $10 / 29$ & & 90 & USW G-2 & .31 & $10 / 29$ & \\
\hline 19 & UE-25 UZN \#23 & .22 & $10 / 29$ & & 55 & USW UZ-N55 & .28 & $10 / 29$ & & 91 & UE25 WT-4 & .24 & $10 / 29$ & \\
\hline 20 & UE-25 UZN \#28 & .19 & $10 / 29$ & & 56 & USW UZ-N57 & $\mathbf{M}^{*}$ & & & 92 & UE25 WT-18 & .35 & $10 / 29$ & \\
\hline 21 & UE-25 UZN \#29 & .22 & $10 / 29$ & & 57 & USW UZ-N64 & $\mathbf{M}^{*}$ & & & 93 & USW H-5 & $\mathbf{M}^{*}$ & & \\
\hline 22 & UE-25 UZN \#30 & .21 & $10 / 29$ & & 58 & USW UZ-N65 & $\mathbf{M}^{*}$ & & & 94 & HRF Wedge & .09 & $10 / 29$ & \\
\hline
\end{tabular}


Table 2. Summary of precipitation data from the nonrecording gage network at Yucca Mountain, Nevada, for water years 1992 and $1993--$ Continued

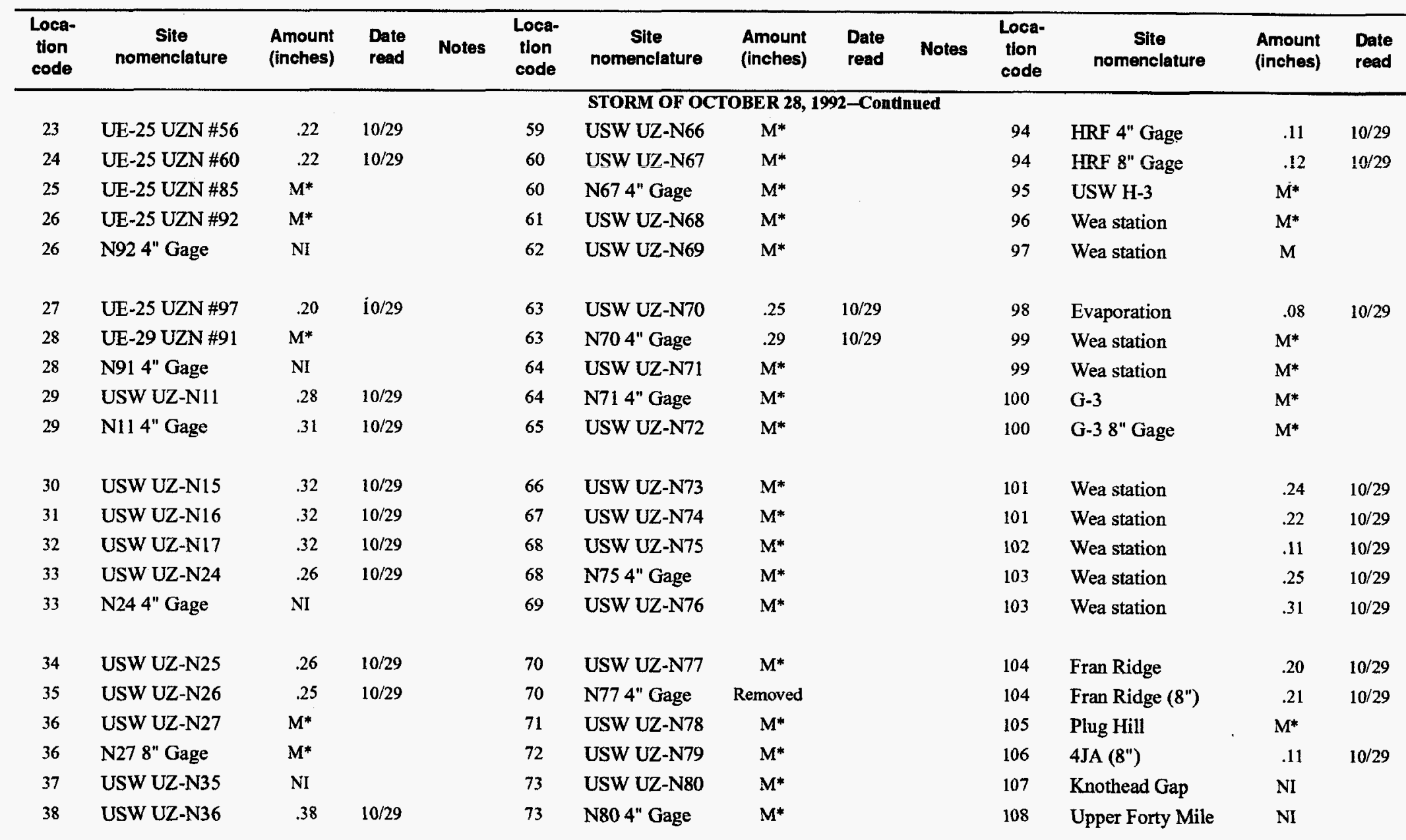


Table 2. Summary of precipitation data from the nonrecording gage network at Yucca Mountain, Nevada, for water years 1992 and $1993--$ Continued

\begin{tabular}{|c|c|c|c|c|c|c|c|c|c|c|c|c|c|c|}
\hline $\begin{array}{l}\text { Loca- } \\
\text { tion } \\
\text { code }\end{array}$ & $\begin{array}{c}\text { Site } \\
\text { nomenclature }\end{array}$ & $\begin{array}{l}\text { Amount } \\
\text { (Inches) }\end{array}$ & $\begin{array}{l}\text { Date } \\
\text { read }\end{array}$ & Notes & $\begin{array}{l}\text { Loca- } \\
\text { tlon } \\
\text { code }\end{array}$ & $\begin{array}{c}\text { Site } \\
\text { nomenclature }\end{array}$ & $\begin{array}{l}\text { Amount } \\
\text { (Inches) }\end{array}$ & $\begin{array}{l}\text { Date } \\
\text { read }\end{array}$ & Notes & $\begin{array}{l}\text { Loca- } \\
\text { tion } \\
\text { code }\end{array}$ & $\begin{array}{c}\text { Site } \\
\text { nomenclature }\end{array}$ & $\begin{array}{l}\text { Amount } \\
\text { (inches) }\end{array}$ & $\begin{array}{l}\text { Date } \\
\text { read }\end{array}$ & Notes \\
\hline \multicolumn{15}{|c|}{ STORM OF OCTOBER 30, 1992} \\
\hline 1 & UE-25 UZN \#1 & .07 & $11 / 4$ & & 39 & USW UZ-N37 & .06 & $11 / 6$ & & 74 & USW UZ-N81 & .17 & $11 / 13$ & *** \\
\hline 2 & UE-25 UZN \#2 & .10 & $11 / 4$ & & 39 & N37 4" Gage & .05 & $11 / 6$ & & 75 & USW UZ-N82 & .17 & $11 / 13$ & ** \\
\hline 3 & UE-25 UZN \#3 & .04 & $11 / 4$ & & 40 & USW UZ-N40 & .04 & $11 / 6$ & & 75 & N82 4" Gage & & & Removed \\
\hline 4 & UE-25 UZN \#4 & .04 & $11 / 4$ & & 40 & N40 4" Gage & NI & & & 76 & USW UZ-N83 & .19 & $11 / 13$ & $* *$ \\
\hline 4 & N4 4" Gage & NI & & & 41 & USW UZ-N41 & .06 & $11 / 6$ & & 77 & USW UZ-N84 & .20 & $11 / 13$ & $* *$ \\
\hline 5 & UE-25 UZN \#5 & .04 & $11 / 4$ & & 42 & USW UZ-N42 & .04 & $11 / 6$ & & 78 & USW UZ-N86 & .20 & $11 / 13$ & *** \\
\hline 6 & UE-25 UZN \#6 & .05 & $11 / 4$ & & 43 & USW UZ-N43 & .05 & $11 / 6$ & & 79 & USW UZ-N87 & .18 & $11 / 13$ & *** \\
\hline 7 & UE-25 UZN \#7 & .03 & $11 / 4$ & & 43 & N43 4" Gage & NI & & & 80 & USW UZ-N88 & .21 & $11 / 13$ & $* *$ \\
\hline 8 & UE-25 UZN \#8 & .04 & $11 / 4$ & & 44 & USW UZ-N44 & .04 & $11 / 6$ & & 81 & USW UZ-N89 & .18 & $11 / 13$ & $* *$ \\
\hline 9 & UE-25 UZN \#9 & .02 & $11 / 4$ & & 45 & USW UZ-N45 & .04 & $11 / 6$ & & 82 & USW UZ-N90 & .18 & $11 / 13$ & $* *$ \\
\hline 10 & UE-25 UZN \#10 & .07 & $11 / 4$ & & 46 & USW UZ-N46 & .069 & $11 / 6$ & & 82 & N90 4" Gage & .21 & $11 / 13$ & $* *$ \\
\hline 10 & N10 4" Gage & NI & & & 47 & USW UZ-N47 & .04 & $11 / 6$ & & 83 & USW UZ-N93 & .26 & $11 / 3$ & *** \\
\hline 11 & UE-25 UZN \#12 & .04 & $11 / 4$ & & 48 & USW UZ-N48 & .02 & $11 / 12$ & & 84 & USW UZ-N94 & .26 & $11 / 3$ & $* *$ \\
\hline 12 & UE-25 UZN \#13 & .04 & $11 / 4$ & & 49 & USW UZ-N49 & .02 & $11 / 12$ & & 85 & USW UZ-N95 & .25 & $11 / 3$ & $* *$ \\
\hline 13 & UE-25 UZN \#14 & .02 & $11 / 4$ & & 50 & USW UZ-N50 & .03 & $11 / 12$ & & 86 & USW UZ-N96 & .23 & $11 / 3$ & $* *$ \\
\hline 14 & UE-25 UZN \#18 & .01 & $11 / 3$ & & 50 & N50 4" Gage & NI & & & 87 & USW UZ-N98 & 0 & $11 / 3$ & \\
\hline 15 & UE-25 UZN \#19 & .05 & $11 / 12$ & & 51 & USW UZ-N51 & .03 & $11 / 12$ & & 87 & N98 4" Gage & $\mathrm{NI}$ & & \\
\hline 16 & UE-25 UZN \#20 & .04 & $11 / 12$ & & 52 & USW UZ-N52 & .01 & $11 / 12$ & & 88 & USW UZ-13 & .19 & $11 / 3$ & $* *$ \\
\hline 17 & UE-25 UZN \#21 & .04 & $11 / 12$ & & 53 & USW UZ-N53 & .03 & $11 / 12$ & & 88 & UZ-13 4" Gage & .22 & $11 / 3$ & $* *$ \\
\hline 17 & N21 4" Gage & NI & & & 54 & USW UZ-N54 & .03 & $11 / 12$ & & 89 & USW GA-1 & .09 & $11 / 4$ & \\
\hline 18 & UE-25 UZN \#22 & .03 & $11 / 12$ & & 54 & N54 4" Gage & $\mathbf{M}$ & & & 90 & USW G-2 & .09 & $11 / 4$ & \\
\hline 19 & UE-25 UZN \#23 & .04 & $11 / 12$ & & 55 & USW UZ-N5S & .02 & $11 / 6$ & & 91 & UE25 WT-4 & .04 & $11 / 4$ & \\
\hline 20 & UE-25 UZN \#28 & .04 & $11 / 12$ & & 56 & USW UZ-N57 & .26 & $11 / 4$ & $* *$ & 92 & UE25 WT-18 & .09 & $11 / 4$ & \\
\hline 21 & UE-25 UZN \#29 & .04 & $11 / 12$ & & 57 & USW UZ-N64 & .30 & $11 / 2$ & $* *$ & 93 & USW H-5 & .26 & $11 / 2$ & $* *$ \\
\hline 22 & UE-25 UZN \#30 & .04 & $11 / 12$ & & 58 & USW UZ-N65 & .22 & $11 / 2$ & $* *$ & 94 & HRF Wedge & 0 & $11 / 6$ & \\
\hline
\end{tabular}


Table 2. Summary of precipitation data from the nonrecording gage network at Yucca Mountain, Nevada, for water years 1992 and $1993--$ Continued

\begin{tabular}{|c|c|c|c|c|c|c|c|c|c|c|c|c|c|c|}
\hline $\begin{array}{l}\text { Loca- } \\
\text { tion } \\
\text { code }\end{array}$ & $\begin{array}{c}\text { Site } \\
\text { nomenclature }\end{array}$ & $\begin{array}{l}\text { Amount } \\
\text { (Inches) }\end{array}$ & $\begin{array}{l}\text { Date } \\
\text { read }\end{array}$ & Notes & $\begin{array}{l}\text { Loca- } \\
\text { tion } \\
\text { code }\end{array}$ & $\begin{array}{c}\text { Site } \\
\text { nomenclature }\end{array}$ & $\begin{array}{l}\text { Amount } \\
\text { (Inches) }\end{array}$ & $\begin{array}{l}\text { Date } \\
\text { read }\end{array}$ & Notes & $\begin{array}{l}\text { Loca- } \\
\text { tion } \\
\text { code }\end{array}$ & $\begin{array}{c}\text { Site } \\
\text { nomenclature }\end{array}$ & $\begin{array}{l}\text { Amount } \\
\text { (Inches) }\end{array}$ & $\begin{array}{l}\text { Date } \\
\text { read }\end{array}$ & Notes \\
\hline \multicolumn{15}{|c|}{ STORM OF OCTOBER 30, 1992-Continued } \\
\hline 23 & UE-25 UZN \#56 & .03 & $11 / 12$ & & 59 & USW UZ-N66 & .25 & $11 / 2$ & *** & 94 & HRF 4" Gage & 0 & $11 / 6$ & \\
\hline 24 & UE-25 UZN \#60 & .03 & $\mathrm{i} 1 / 12$ & & 60 & USWW UZ-N67 & .20 & $11 / 2$ & ** & 94 & HRF 8" Gage & 0 & $11 / 6$ & \\
\hline 25 & UE-25 UZN \#85 & .29 & $11 / 2$ & ** & 60 & N67 4" Gage & .22 & $11 / 2$ & ** & 95 & USW H-3 & .22 & $11 / 2$ & \\
\hline 26 & UE-25 UZN \#92 & .18 & $11 / 2$ & $* *$ & 61 & USW UZ-N68 & .21 & $11 / 2$ & $* *$ & 96 & Wea station & .26 & $11 / 2$ & \\
\hline 26 & N92 4" Gage & NI & & & 62 & USW UZ-N69 & .20 & $11 / 2$ & $* *$ & 97 & Wea station & M & & \\
\hline 27 & UE-25 UZN \#97 & .02 & $11 / 2$ & & 63 & USW UZ-N70 & .03 & $11 / 2$ & & 98 & Evaporation & 0 & $11 / 6$ & \\
\hline 28 & UE-29 UZN \#91 & .28 & $11 / 2$ & ** & 63 & N70 4" Gage & .03 & $11 / 2$ & & 99 & Wea station & .29 & $11 / 3$ & ** \\
\hline 28 & N91 4" Gage & $\mathrm{NI}$ & & & 64 & USW UZ-N71 & .26 & $11 / 2$ & ** & 99 & Wea station & .27 & $11 / 3$ & $* *$ \\
\hline 29 & USW UZ-N1I & .08 & $11 / 4$ & & 64 & N71 4" Gage & .29 & $11 / 2$ & $* *$ & 100 & $\mathrm{G}-3$ & .21 & $11 / 3$ & $* *$ \\
\hline 29 & N11 4" Gage & .10 & $11 / 4$ & & 65 & USW UZ-N72 & .28 & $11 / 2$ & $* *$ & 100 & G-3 8" Gage & .23 & $11 / 3$ & $* *$ \\
\hline 30 & USW UZ-N15 & .09 & $11 / 4$ & & 66 & USW UZ-N73 & .27 & $11 / 2$ & ** & 101 & Wea station & .01 & $11 / 6$ & \\
\hline 31 & USW UZ-N16 & .08 & $11 / 4$ & & 67 & USW UZ-N74 & .26 & $11 / 2$ & ** & 101 & Wea station & .01 & $11 / 6$ & \\
\hline 32 & USW UZ-N17 & .08 & $11 / 4$ & & 68 & USW UZ-N75 & .24 & $11 / 2$ & $* *$ & 102 & Wea station & .08 & $11 / 6$ & \\
\hline 33 & USW UZ-N24 & .04 & $11 / 6$ & & 68 & N75 4" Gage & .31 & $11 / 2$ & $* *$ & 103 & Wea station & .03 & $11 / 6$ & \\
\hline 33 & N24 4" Gage & NI & & & 69 & USW UZ-N76 & .30 & $11 / 2$ & $* *$ & 103 & Wea station & M & & \\
\hline 34 & USW UZ-N25 & .04 & $11 / 6$ & & 70 & USW UZ-N77 & .17 & $11 / 13$ & ** & 104 & Fran Ridge & 0 & $11 / 6$ & \\
\hline 35 & USW UZ-N26 & .04 & $11 / 6$ & & 70 & N77 4" Gage & Removed & & & 104 & Fran Ridge (8") & .01 & & \\
\hline 36 & USW UZ-N27 & .33 & $11 / 2$ & $* *$ & 71 & USW UZ-N78 & .29 & $11 / 13$ & $* *$ & 105 & Plug Hill & .14 & $11 / 13$ & \\
\hline 36 & N27 8" Gage & .39 & $11 / 2$ & $* *$ & 72 & USW UZ-N79 & .31 & $11 / 13$ & $* *$ & 106 & 4JA (8") & 0 & & \\
\hline 37 & USW UZ-N35 & NI & & & 73 & USW UZ-N80 & .31 & $11 / 13$ & $* *$ & 107 & Knothead Gap & NI & & \\
\hline 38 & USW UZ-N36 & .10 & $11 / 4$ & & 73 & N80 4" Gage & .30 & $11 / 13$ & $* *$ & 108 & Upper Forty Mile & NI & & \\
\hline
\end{tabular}


Table 2. Summary of precipitation data from the nonrecording gage network at Yucca Mountain, Nevada, for water years 1992 and 1993--Continued

\begin{tabular}{|c|c|c|c|c|c|c|c|c|c|c|c|c|c|c|}
\hline $\begin{array}{l}\text { Loca- } \\
\text { tion } \\
\text { code }\end{array}$ & $\begin{array}{c}\text { Site } \\
\text { nomenclature }\end{array}$ & $\begin{array}{l}\text { Amount } \\
\text { (Inches) }\end{array}$ & $\begin{array}{l}\text { Date } \\
\text { read }\end{array}$ & Notes & $\begin{array}{l}\text { Loca- } \\
\text { tion } \\
\text { code }\end{array}$ & $\begin{array}{c}\text { Site } \\
\text { nomenclature }\end{array}$ & $\begin{array}{l}\text { Amount } \\
\text { (inches) }\end{array}$ & $\begin{array}{l}\text { Date } \\
\text { read }\end{array}$ & Notes & $\begin{array}{l}\text { Loca- } \\
\text { tion } \\
\text { code }\end{array}$ & $\begin{array}{c}\text { Site } \\
\text { nomenclature }\end{array}$ & $\begin{array}{l}\text { Amount } \\
\text { (inches) }\end{array}$ & $\begin{array}{l}\text { Date } \\
\text { read }\end{array}$ & Notes \\
\hline \multicolumn{15}{|c|}{ STORM OF DECEMBER 7-8, 1992} \\
\hline 1 & UE-25 UZN \#1 & 2.28 & $12 / 10$ & & 39 & USW UZ-N37 & 2.25 & $12 / 10$ & & 74 & USW UZ-N81 & 2.55 & $12 / 11$ & \\
\hline 2 & UE-25 UZN \#2 & 2.40 & $12 / 10$ & & 39 & N37 4" Gage & 2.31 & $12 / 10$ & & 75 & USW UZ-N82 & 2.52 & $12 / 11$ & \\
\hline 3 & UE-25 UZN \#3 & 2.20 & $12 / 10$ & & 40 & USW UZ-N40 & 2.13 & $12 / 11$ & & 75 & N82 4" Gage & & & Removed \\
\hline 4 & UE-25 UZN \#4 & 2.25 & $12 / 10$ & & 40 & N40 4" Gage & NI & & & 76 & USW UZ-N83 & 2.65 & $12 / 11$ & \\
\hline 4 & N4 4" Gage & NI & & & 41 & USW UZ-N41 & 2.22 & $12 / 9$ & & 77 & USW UZ-N84 & 2.38 & $12 / 11$ & \\
\hline 5 & UE-25 UZN \#5 & 2.20 & $12 / 10$ & & 42 & USW UZ-N42 & 2.23 & $12 / 11$ & & 78 & USW UZ-N86 & 2.53 & $12 / 11$ & \\
\hline 6 & UE-25 UZN \#6 & 2.25 & $12 / 10$ & & 43 & USW UZ-N43 & 2.30 & $12 / 11$ & & 79 & USW UZ-N87 & 2.40 & $12 / 11$ & \\
\hline 7 & UE-25 UZN \#7 & 2.16 & $12 / 10$ & & 43 & N43 4" Gage & NI & & & 80 & USW UZ-N88 & 2.55 & $12 / 11$ & $@$ \\
\hline 8 & UE-25 UZN \#8 & 2.20 & $12 / 10$ & & 44 & USW UZ-N44 & 2.33 & $12 / 11$ & & 81 & USW UZ-N89 & 2.41 & $12 / 11$ & \\
\hline 9 & UE-25 UZN \#9 & 2.13 & $12 / 10$ & & 45 & USW UZ-N45 & 2.40 & $12 / 11$ & & 82 & USW UZ-N90 & 2.47 & $12 / 11$ & \\
\hline 10 & UE-25 UZN \#10 & 2.20 & $12 / 10$ & & 46 & USW UZ-N46 & 1.94 & $12 / 10$ & & 82 & N90 4" Gage & 2.66 & $12 / 11$ & \\
\hline 10 & N10 4" Gage & NI & & & 47 & USW UZ-N47 & 2.03 & $12 / 10$ & & 83 & USW UZ-N93 & 1.45 & $12 / 9$ & \\
\hline 11 & UE-25 UZN \#12 & 2.25 & $12 / 10$ & & 48 & USW UZ-N48 & 2.40 & $12 / 11$ & & 84 & USW UZ-N94 & 1.60 & $12 / 9$ & \\
\hline 12 & UE-25 UZN \#13 & 2.31 & $12 / 10$ & & 49 & USW UZ-N49 & 2.06 & $12 / 11$ & & 85 & USW UZ-N95 & 1.45 & $12 / 9$ & \\
\hline 13 & UE-25 UZN \#14 & 2.25 & $12 / 10$ & & 50 & USW UZ-N50 & 2.10 & $12 / 11$ & & 86 & USW UZ-N96 & 1.28 & $12 / 9$ & \\
\hline 14 & UE-25 UZN \#18 & 2.25 & $12 / 10$ & & 50 & N50 4" Gage & $\mathrm{NI}$ & & & 87 & USW UZ-N98 & 2.47 & $12 / 10$ & \\
\hline 15 & UE-25 UZN\#19 & 2.19 & $12 / 10$ & & 51 & USW UZ-N51 & 2.11 & $12 / 11$ & & 87 & N98 4" Gage & NI & & \\
\hline 16 & UE-25 UZN \#20 & 2.25 & $12 / 11$ & & 52 & USW UZ-N52 & 2.11 & $12 / 11$ & & 88 & USW UZ-13 & 1.18 & $12 / 9$ & \\
\hline 17 & UE-25 UZN \#21 & 2.20 & $12 / 11$ & & 53 & USW UZ-N53 & 2.20 & $12 / 11$ & & 88 & UZ-13 4" Gage & 1.48 & $12 / 9$ & \\
\hline 17 & N21 4" Gage & NI & & & 54 & USW UZ-N54 & 1.75 & $12 / 11$ & & 89 & USW GA-1 & .75 & $12 / 10$ & \\
\hline 18 & UE-25 UZN \#22 & 2.21 & $12 / 11$ & & 54 & N54 4" Gage & 2.30 & $12 / 11$ & & 90 & USW G-2 & .82 & $12 / 10$ & \\
\hline 19 & UE-25 UZN \#23 & 2.20 & $12 / 11$ & & 55 & USW UZ-N55 & 2.11 & $12 / 11$ & & 91 & UE25 WT-4 & 2.26 & $12 / 10$ & \\
\hline 20 & UE-25 UZN \#28 & 2.20 & $12 / 11$ & & 56 & USW UZ-N57 & 2.60 & $12 / 9$ & & 92 & UE25 WT-18 & 1.74 & $12 / 10$ & \\
\hline 21 & UE-25 UZN \#29 & 2.15 & $12 / 11$ & & 57 & USW UZ-N64 & 1.56 & $12 / 9$ & & 93 & USW H-5 & 1.15 & $12 / 9$ & \\
\hline 22 & UE-25 UZN \#30 & 2.23 & $12 / 11$ & & 58 & USW UZ-N65 & 1.94 & $12 / 9$ & & 94 & HRF Wedge & 1.38 & $12 / 10$ & \\
\hline
\end{tabular}


Table 2. Summary of precipitation data from the nonrecording gage network at Yucca Mountain, Nevada, for water years 1992 and 1993 --Continued

\begin{tabular}{|c|c|c|c|c|c|c|c|c|c|c|c|c|c|c|}
\hline $\begin{array}{l}\text { Loca- } \\
\text { tion } \\
\text { code }\end{array}$ & $\begin{array}{c}\text { Site } \\
\text { nomenclature }\end{array}$ & $\begin{array}{l}\text { Amount } \\
\text { (Inches) }\end{array}$ & $\begin{array}{l}\text { Date } \\
\text { read }\end{array}$ & Notes & $\begin{array}{l}\text { Loca- } \\
\text { tion } \\
\text { code }\end{array}$ & $\begin{array}{c}\text { Site } \\
\text { nomenclature }\end{array}$ & $\begin{array}{l}\text { Amount } \\
\text { (inches) }\end{array}$ & $\begin{array}{l}\text { Date } \\
\text { read }\end{array}$ & Notes & $\begin{array}{l}\text { Loca- } \\
\text { tlon } \\
\text { code }\end{array}$ & $\begin{array}{c}\text { Site } \\
\text { nomenclature }\end{array}$ & $\begin{array}{l}\text { Amount } \\
\text { (Inches) }\end{array}$ & $\begin{array}{l}\text { Date } \\
\text { read }\end{array}$ & Notes \\
\hline \multicolumn{15}{|c|}{ STORM OF DECEMBER 7-8, 1992-Continued } \\
\hline 23 & UE-25 UZN \#56 & 2.25 & $12 / 11$ & & 59 & USW UZ-N66 & 1.83 & $12 / 9$ & & 94 & HRF 4" Gage & 1.36 & $12 / 10$ & \\
\hline 24 & UE-25 UZN \#GO & 2.25 & $12 / 11$ & & 60 & USW UZ-N67 & 2.26 & $12 / 9$ & & 94 & HRF 8" Gage & 1.43 & $12 / 10$ & \\
\hline 25 & UE-25 UŻN \#85 & 1.90 & $12 / 8$ & & 60 & N67 4" Gage & 1.38 & $12 / 9$ & & 95 & USW H-3 & .73 & $12 / 9$ & \\
\hline 26 & UE-25 UZN \#92 & 2.15 & $12 / 9$ & & 61 & USW UZ-N68 & 2.24 & $12 / 9$ & & 96 & Wea station & .95 & $12 / 9$ & \\
\hline 26 & N92 4" Gage & 2.16 & $12 / 9$ & & 62 & USW UZ-N69 & 2.24 & $12 / 9$ & & 97 & Wea station & $\mathbf{M}$ & & \\
\hline 27 & UE-25 UZN \#97 & 2.20 & $12 / 11$ & & 63 & USW UZ-N70 & 1.65 & $12 / 10$ & & 98 & Evaporation & 1.78 & $12 / 9$ & \\
\hline 28 & UE-29 UZN \#91 & 2.11 & $12 / 9$ & & 63 & N70 4" Gage & 1.76 & $12 / 10$ & & 99 & Wea station & $\mathbf{M}$ & & \\
\hline 28 & N91 4" Gage & 1.99 & $12 / 9$ & & 64 & USW UZ-N71 & 1.05 & $12 / 9$ & & 99 & Wea station & 1.46 & $12 / 9$ & \\
\hline 29 & USW UZ-N11 & .65 & $12 / 9$ & & 64 & N71 4" Gage & 1.11 & $12 / 9$ & & 100 & G-3 & 1.61 & $12 / 9$ & \\
\hline 29 & N11 4" Gage & .75 & $12 / 9$ & & 65 & USW UZ-N72 & $\mathbf{M}^{*}$ & & & 100 & G-3 8" Gage & 1.91 & $12 / 9$ & \\
\hline 30 & USW UZ-N15 & .95 & $12 / 9$ & & 66 & USW UZ-N73 & $\mathbf{M}^{*}$ & & & 101 & Wea station & 2.18 & $12 / 11$ & \\
\hline 31 & USW UZ-N16 & 1.21 & $12 / 9$ & & 67 & USW UZ-N74 & 1.03 & $12 / 9$ & & 101 & Wea station & 2.18 & $12 / 11$ & \\
\hline 32 & USW UZ-N17 & .74 & $12 / 9$ & & 68 & USW UZ-N75 & $\mathbf{M}^{*}$ & & & 102 & Wea station & 1.85 & $12 / 10$ & \\
\hline 33 & USW UZ-N24 & 2.27 & $12 / 10$ & & 68 & N75 4" Gage & $\mathbf{M}^{*}$ & & & 103 & Wea station & 2.03 & $12 / 10$ & \\
\hline 33 & N24 4" Gage & NI & & & 69 & USW UZ-N76 & $\mathrm{M}^{*}$ & & & 103 & Wea station & 2.30 & $12 / 10$ & \\
\hline 34 & USW UZ-N25 & 2.45 & $12 / 10$ & & 70 & USW UZ-N77 & 2.63 & $12 / 11$ & & 104 & Fran Ridge & 1.75 & $12 / 9$ & \\
\hline 35 & USW UZ-N26 & 2.20 & $12 / 10$ & & 70 & N77 4" Gage & Removed & & & 104 & Fran Ridge (8") & 1.76 & $12 / 9$ & \\
\hline 36 & USW UZ-N27 & .78 & $12 / 9$ & & 71 & USW UZ-N78 & 2.45 & $12 / 11$ & & 105 & Plug Hill & 2.05 & $12 / 11$ & \\
\hline 37 & USW UZ-N35 & 2.33 & $12 / 11$ & & 73 & USW UZ-N80 & 2.40 & $12 / 11$ & & 107 & Knothead Gap & 2.26 & $12 / 9$ & \\
\hline 38 & USW UZ-N36 & 1.65 & $12 / 9$ & & 73 & N80 4" Gage & 2.60 & $12 / 11$ & & 108 & Upper Forty Mile & 2.05 & $12 / 9$ & \\
\hline
\end{tabular}


Table 2. Summary of precipitation data from the nonrecording gage network at Yucca Mountain, Nevada, for water years 1992 and $1993--$ Continued

\begin{tabular}{|c|c|c|c|c|c|c|c|c|c|c|c|c|c|c|}
\hline $\begin{array}{l}\text { Loca- } \\
\text { tion } \\
\text { code }\end{array}$ & $\begin{array}{c}\text { Site } \\
\text { nomenclature }\end{array}$ & $\begin{array}{l}\text { Amount } \\
\text { (inches) }\end{array}$ & $\begin{array}{l}\text { Date } \\
\text { read }\end{array}$ & Notes & $\begin{array}{l}\text { Loca- } \\
\text { tion } \\
\text { code }\end{array}$ & $\begin{array}{c}\text { Site } \\
\text { nomenclature }\end{array}$ & $\begin{array}{l}\text { Amount } \\
\text { (inches) }\end{array}$ & $\begin{array}{l}\text { Date } \\
\text { read }\end{array}$ & Notes & $\begin{array}{l}\text { Loca- } \\
\text { tion } \\
\text { code }\end{array}$ & $\begin{array}{c}\text { Site } \\
\text { nomenclature }\end{array}$ & $\begin{array}{l}\text { Amount } \\
\text { (inches) }\end{array}$ & $\begin{array}{l}\text { Date } \\
\text { read }\end{array}$ & Notes \\
\hline \multicolumn{15}{|c|}{ STORM OF DECEMBER 11-12,1992 } \\
\hline 1 & UE-25 UZN \#1 & $\mathbf{M}^{*}$ & & & 39 & USW UZ-N37 & .03 & $12 / 17$ & & 74 & USW UZ-N81 & $\mathbf{M}^{*}$ & & \\
\hline 2 & UE-25 UZN \#2 & .05 & $12 / 15$ & & 39 & N37 4" Gage & .05 & $12 / 17$ & & 75 & USW UZ-N82 & $\mathbf{M}^{*}$ & & \\
\hline 3 & UE-25 UZN \#3 & .08 & $12 / 14$ & & 40 & USW UZ-N40 & .05 & $12 / 16$ & & 75 & N82 4" Gage & & & Removed \\
\hline 4 & UE-25 UZN \#4 & .05 & $12 / 14$ & & 40 & N40 4" Gage & NI & & & 76 & USW UZ-N83 & $\mathbf{M}^{*}$ & & \\
\hline 4 & N4 4" Gage & NI & & & 41 & USW UZ-N41 & .02 & $12 / 16$ & & 77 & USW UZ-N84 & $\mathbf{M}^{*}$ & & \\
\hline 5 & UE-25 UZN \#5 & .08 & $12 / 14$ & & 42 & USW UZ-N42 & .02 & $12 / 16$ & & 78 & USW UZ-N86 & $\mathbf{M}^{*}$ & & \\
\hline 6 & UE-25 UZN \#6 & .06 & $12 / 14$ & & 43 & USW UZ-N43 & .02 & $12 / 16$ & & 79 & USW UZ-N87 & $\mathrm{M}^{*}$ & & \\
\hline 7 & UE-25 UZN \#7 & .05 & $12 / 14$ & & 43 & N43 4" Gage & $\mathrm{NI}$ & & & 80 & USW UZ-N88 & $\mathrm{M}^{*}$ & & \\
\hline 8 & UE-25 UZN \#8 & $\mathbf{M}^{*}$ & & & 44 & USW UZ-N44 & .01 & $12 / 16$ & & 81 & USW UZ-N89 & $\mathbf{M}^{*}$ & & \\
\hline 9 & UE-25 UZN \#9 & .09 & $12 / 14$ & & 45 & USW UZ-N45 & .02 & $12 / 16$ & & 82 & USW UZ-N90 & $\mathrm{M}^{*}$ & & \\
\hline 10 & UE-25 UZN \#10 & .12 & $12 / 14$ & & 46 & USW UZ-N46 & 0 & $12 / 14$ & & 82 & N90 4" Gage & $\mathbf{M}^{*}$ & & \\
\hline 10 & N10 4" Gage & NI & & & 47 & USW UZ-N47 & .02 & $12 / 14$ & & 83 & USW UZ-N93 & $\mathbf{M}^{*}$ & & \\
\hline 11 & UE-25 UZN \#12 & $\mathbf{M}^{*}$ & & & 48 & USW UZ-N48 & $\mathrm{M}^{*}$ & & & 84 & USW UZ-N94 & $\mathbf{M}^{*}$ & & \\
\hline 12 & UE-25 UZN \#13 & $\mathbf{M}^{*}$ & & & 49 & USW UZ-N49 & $\mathrm{M}^{*}$ & & & 85 & USW UZ-N95 & $\mathbf{M}^{*}$ & & \\
\hline 13 & UE-25 UZN \#14 & $\mathbf{M}^{*}$ & & & 50 & USW UZ-N50 & $\mathrm{M}^{*}$ & & & 86 & USW UZ-N96 & $\mathbf{M}^{*}$ & & \\
\hline 14 & UE-25 UZN \#18 & .04 & $12 / 14$ & & 50 & N50 4" Gage & NI & & & 87 & USW UZ-N98 & $\mathbf{M}^{*}$ & & \\
\hline 15 & UE-25 UZN \#19 & .03 & $12 / 14$ & & 51 & USW UZ-N51 & $\mathbf{M}^{*}$ & & & 87 & N98 4" Gage & NI & & \\
\hline 16 & UE-25 UZN \#20 & .03 & $12 / 17$ & & 52 & USW UZ-N52 & $\mathrm{M}^{*}$ & & & 88 & USW UZ-13 & $\mathbf{M}^{*}$ & & \\
\hline 17 & UE-25 UZN \#21 & .04 & $12 / 17$ & & 53 & USW UZ-N53 & .08 & $12 / 15$ & & 88 & UZ-13 4" Gage & $\mathbf{M}$ & & \\
\hline 17 & N21 4" Gage & NI & & & 54 & USW UZ-N54 & .05 & $12 / 15$ & & 89 & USW GA-1 & $\mathbf{M}$ & & \\
\hline 18 & UE-25 UZN \#22 & .05 & $12 / 17$ & & 54 & N54 4" Gage & .06 & $12 / 15$ & & 90 & USW G-2 & 0 & $12 / 14$ & \\
\hline 19 & UE-25 UZN \#23 & .03 & $12 / 17$ & & 55 & USW UZ-N55 & .05 & $12 / 15$ & & 91 & UE25 WT-4 & .07 & $12 / 14$ & \\
\hline 20 & UE-25 UZN \#28 & $\mathbf{M}^{*}$ & & & 56 & USW UZ-N57 & .03 & $12 / 17$ & & 92 & UE25 WT-18 & 0 & $12 / 14$ & \\
\hline 21 & UE-25 UZN \#29 & $\mathbf{M}^{*}$ & & & 57 & USW UZ-N64 & M & & & 93 & USW H-5 & $\mathbf{M}^{*}$ & & \\
\hline 22 & UE-25 UZN \#30 & $\mathrm{M}^{*}$ & & & 58 & USW UZ-N65 & $\mathbf{M}$ & & & 94 & HRF Wedge & .05 & $12 / 14$ & \\
\hline
\end{tabular}


Table 2. Summary of precipitation data from the nonrecording gage network at Yucca Mountain, Nevada, for water years 1992 and 1993 --Continued

\begin{tabular}{|c|c|c|c|c|c|c|c|c|c|c|c|c|c|c|}
\hline $\begin{array}{l}\text { Loca- } \\
\text { tion } \\
\text { code }\end{array}$ & $\begin{array}{c}\text { Site } \\
\text { nomenclature }\end{array}$ & $\begin{array}{l}\text { Amount } \\
\text { (inches) }\end{array}$ & $\begin{array}{l}\text { Date } \\
\text { read }\end{array}$ & Notes & $\begin{array}{l}\text { Loca- } \\
\text { tion } \\
\text { code }\end{array}$ & $\begin{array}{c}\text { Site } \\
\text { nomenclature }\end{array}$ & $\begin{array}{l}\text { Amount } \\
\text { (inches) }\end{array}$ & $\begin{array}{l}\text { Date } \\
\text { read }\end{array}$ & Notes & $\begin{array}{l}\text { Loca- } \\
\text { tion } \\
\text { code }\end{array}$ & $\begin{array}{c}\text { Site } \\
\text { nomenclature }\end{array}$ & $\begin{array}{l}\text { Amount } \\
\text { (inches) }\end{array}$ & $\begin{array}{l}\text { Date } \\
\text { read }\end{array}$ & Notes \\
\hline \multicolumn{15}{|c|}{ STORM OF DECEMBER 11-12, 1992-Contnued } \\
\hline 23 & UE-25 UZN \#56 & $\mathbf{M}^{*}$ & & & 59 & USW UZ-N66 & M & & & 94 & HRF 4" Gage & .07 & $12 / 14$ & \\
\hline 24 & UE-25 UZN \#60 & $\mathrm{M}^{*}$ & & & 60 & USW UZ-N67 & .01 & $12 / 15$ & & 94 & HRF 8" Gage & .07 & $12 / 14$ & \\
\hline 25 & UE-25 UZN \#85 & .06 & $12 / 15$ & & 60 & N67 4" Gage & .09 & $12 / 15$ & & 95 & USW H-3 & $\mathbf{M}^{*}$ & & \\
\hline 26 & UE-25 UZN \#92 & .10 & $12 / 14$ & & 61 & USW UZ-N68 & .06 & $12 / 15$ & & 96 & Wea station & $\mathrm{M}^{*}$ & & \\
\hline 26 & N92 4" Gage & .07 & $12 / 14$ & & 62 & USW UZ-N69 & .07 & $12 / 15$ & & 97 & Wea station & $\mathbf{M}$ & & \\
\hline 27 & UE-25 UZN \#97 & .15 & $12 / 22$ & & 63 & USW UZ-N70 & $\mathrm{M}^{*}$ & & & 98 & Evaporation & .06 & $12 / 14$ & \\
\hline 28 & UE-29 UZN \#91 & .05 & $12 / 14$ & & 63 & N70 4" Gage & $\mathbf{M}$ & & & 99 & Wea station & $M$ & & \\
\hline 28 & N91 4" Gage & .07 & $12 / 14$ & & 64 & USW UZ-N71 & .05 & $12 / 15$ & & 99 & Wea station & M & & \\
\hline 29 & USW UZ-N11 & .02 & $12 / 14$ & & 64 & N71 4" Gage & $\mathrm{M}$ & & & 100 & G-3 & $\mathbf{M}^{*}$ & & \\
\hline 29 & N11 4" Gage & 0 & $12 / 14$ & & 65 & USW UZ-N72 & 1.01 & $12 / 15$ & $* *$ & 100 & G.3 8" Gage & $\mathrm{M}^{*}$ & & \\
\hline 30 & USW UZ-N15 & 0 & $12 / 14$ & & 66 & USW UZ-N73 & .95 & $12 / 15$ & $* *$ & 101 & Wea station & .15 & & \\
\hline 31 & USW UZ-N16 & 0 & $12 / 14$ & & 67 & USW UZ-N74 & $\mathbf{M}$ & & & 101 & Wea station & .09 & & \\
\hline 32 & 'USW UZ-N17 & 0 & $12 / 14$ & & 68 & USW UZ-N75 & 1.30 & $12 / 15$ & $* *$ & 102 & Wea station & $\mathrm{M}^{*}$ & & \\
\hline 33 & USW UZ-N24 & $\mathrm{M}^{*}$ & & & 68 & N75 4" Gage & 1.21 & $12 / 15$ & $* *$ & 103 & Wea station & .01 & $12 / 14$ & \\
\hline 33 & N24 4" Gage & NI & & & 69 & USW UZ-N76 & 1.25 & $* *$ & & 103 & Wea station & .01 & $12 / 14$ & \\
\hline 34 & USW UZ-N25 & $\mathrm{M}^{*}$ & & & 70 & USW UZ-N77 & $\mathrm{M}^{*}$ & & & 104 & Fran Ridge & $\mathrm{M}^{*}$ & & \\
\hline 35 & USW UZ-N26 & $\mathrm{M}^{*}$ & & & 70 & N77 4" Gage & Removed & & & 104 & Fran Ridge (8") & $\mathrm{M}^{*}$ & & \\
\hline 36 & USW UZ-N27 & 0 & $12 / 14$ & & 71 & USW UZ-N78 & $\mathbf{M}^{*}$ & & & 105 & Plug Hill & $\mathbf{M}^{*}$ & & \\
\hline 36 & N27 8" Gage & 0 & $12 / 14$ & & 72 & USW UZ-N79 & $\mathbf{M}^{*}$ & & & 106 & $4 \mathrm{JA}\left(8^{\prime \prime}\right)$ & .05 & $12 / 14$ & \\
\hline 37 & USW UZ-N35 & .05 & $12 / 22$ & & 73 & USW UZ-N80 & $\mathrm{M}^{*}$ & & & 107 & Knothead Gap & $\mathrm{M}^{*}$ & & \\
\hline 38 & USW UZ-N36 & 0 & $12 / 14$ & & 73 & N80 4" Gage & $\mathrm{M}^{*}$ & & & 108 & Upper Forty Mile & $\mathrm{M}^{*}$ & & \\
\hline
\end{tabular}


Table 2. Summary of precipitation data from the nonrecording gage network at Yucca Mountain, Nevada, for water years 1992 and $1993-$-Continued

\begin{tabular}{|c|c|c|c|c|c|c|c|c|c|c|c|c|c|c|}
\hline $\begin{array}{l}\text { Loca- } \\
\text { tion } \\
\text { code }\end{array}$ & $\begin{array}{c}\text { Site } \\
\text { nomenclature }\end{array}$ & $\begin{array}{l}\text { Amount } \\
\text { (inches) }\end{array}$ & $\begin{array}{l}\text { Date } \\
\text { read }\end{array}$ & Notes & $\begin{array}{l}\text { Loca- } \\
\text { tion } \\
\text { code }\end{array}$ & $\begin{array}{c}\text { Site } \\
\text { nomenclature }\end{array}$ & $\begin{array}{l}\text { Amount } \\
\text { (inches) }\end{array}$ & $\begin{array}{l}\text { Date } \\
\text { read }\end{array}$ & Notes & $\begin{array}{l}\text { Loca- } \\
\text { tion } \\
\text { code }\end{array}$ & $\begin{array}{c}\text { Site } \\
\text { nomenclature }\end{array}$ & $\begin{array}{l}\text { Amount } \\
\text { (Inches) }\end{array}$ & $\begin{array}{l}\text { Date } \\
\text { read }\end{array}$ & Notes \\
\hline \multicolumn{15}{|c|}{ STORM OF DECEMBER 18, 1992} \\
\hline 1 & UE-25 UZN \#1 & .12 & $12 / 22$ & ** & 39 & USW UZ-N37 & $\mathbf{M}^{*}$ & & & 74 & USW UZ-N81 & $\mathbf{M}^{*}$ & & \\
\hline 2 & UE-25 UZN \#2 & $\mathbf{M}^{*}$ & & & 39 & N37 4" Gage & $\mathbf{M}^{*}$ & & & 75 & USW UZ-N82 & $\mathbf{M}^{*}$ & & \\
\hline 3 & UE-25 UZN \#3 & .02 & $12 / 22$ & & 40 & USW UZ-N40 & $\mathbf{M}^{*}$ & & & 75 & N82 4" Gage & & & Removed \\
\hline 4 & UE-25 UZN \#4 & .03 & $12 / 22$ & & 40 & N40 4" Gage & NI & & & 76 & USW UZ-N83 & $\mathbf{M}^{*}$ & & \\
\hline 4 & N4 4" Gage & NI & & & 41 & USW UZ-N41 & $\mathbf{M}^{*}$ & & & 77 & USW UZ-N84 & $\mathbf{M}^{*}$ & & \\
\hline 5 & UE-25 UZN \#5 & $\mathbf{M}^{*}$ & & & 42 & USW UZ-N42 & $\mathbf{M}^{*}$ & & & 78 & USW UZ-N86 & $\mathbf{M}^{*}$ & & \\
\hline 6 & UE-25 UZN \#6 & $\mathbf{M}^{*}$ & & & 43 & USW UZ-N43 & $\mathbf{M}^{*}$ & & & 79 & USW UZ-N87 & $\mathbf{M}^{*}$ & & \\
\hline 7 & UE-25 UZN \#7 & .03 & $12 / 22$ & & & N43 4" Gage & NI & & & 80 & USW UZ-N88 & $\mathbf{M}^{*}$ & & \\
\hline 8 & UE-25 UZN \#8 & $\mathbf{M}^{*}$ & & & 43 & USW UZ-N44 & $\mathbf{M}^{*}$ & & & 81 & USW UZ-N89 & $\mathbf{M}^{*}$ & & \\
\hline 9 & UE-25 UZN \#9 & .03 & $12 / 22$ & & 44 & USW UZ-N45 & $\mathbf{M}^{*}$ & & & 82 & USW UZ-N90 & $\mathbf{M}^{*}$ & & \\
\hline 10 & UE-25 UZN \#10 & 0 & $12 / 22$ & & 45 & USW UZ-N46 & $\mathbf{M}^{*}$ & & & 82 & N90 4" Gage & $\mathbf{M}^{*}$ & & \\
\hline 10 & N10 4" Gage & NI & & & 46 & USW UZ-N47 & $\mathbf{M}^{*}$ & & & 83 & USW UZ-N93 & $\mathbf{M}^{*}$ & & \\
\hline 11 & UE-25 UZN \#12 & .12 & $12 / 21$ & $* *$ & 47 & USW UZ-N48 & $\mathbf{M}^{*}$ & & & 84 & USW UZ-N94 & $\mathbf{M}^{*}$ & & \\
\hline 12 & UE-25 UZN \#13 & $\mathbf{M}^{*}$ & & & 48 & USW UZ-N49 & $\mathbf{M}^{*}$ & & & 85 & USW UZ-N95 & $\mathbf{M}^{*}$ & & \\
\hline 13 & UE-25 UZN \#14 & $\mathbf{M}^{*}$ & & & 49 & USW UZ-N50 & $\mathbf{M}^{*}$ & & & 86 & USW UZ-N96 & $\mathbf{M}^{*}$ & & \\
\hline 14 & UE-25 UZN \#18 & $\mathbf{M}^{*}$ & & & 50 & N50 4" Gage & NI & & & 87 & USW UZ-N98 & $\mathbf{M}^{*}$ & & \\
\hline 15 & UE-25 UZN \#19 & 0 & $12 / 18$ & & 50 & USW UZ-N51 & $\mathbf{M}^{*}$ & & & 87 & N98 4" Gage & NI & & \\
\hline 16 & UE-25 UZN\#20 & 0 & $12 / 18$ & & 51 & USW UZ-N52 & $\mathbf{M}^{*}$ & & & 88 & USW UZ-13 & $\mathbf{M}^{*}$ & & \\
\hline 17 & $\mathrm{UE}-25 \mathrm{UZN} \# 21$ & 0 & $12 / 18$ & & 52 & · USW UZ-N53 & $\mathbf{M}^{*}$ & & & 88 & UZ-13 4" Gage & $\mathbf{M}$ & & \\
\hline 17 & N21 4" Gage & NI & & & 53 & USW UZ-N54 & $\mathrm{M}^{*}$ & & & 89 & USW GA-1 & $\mathbf{M}$ & & \\
\hline 18 & UE-25 UZN \#22 & 0 & $12 / 18$ & & 54 & N54 4" Gage & $\mathbf{M}^{*}$ & & & 90 & USW G-2 & $\mathbf{M}$ & & \\
\hline 19 & UE-25 UZN \#23 & 0 & $12 / 18$ & & 54 & USW UZ-N55 & $\mathbf{M}^{*}$ & & & 91 & UE25 WT-4 & .07 & $12 / 22$ & \\
\hline 20 & UE-25 UZN \#28 & .15 & $12 / 22$ & $* *$ & 55 & USW UZ-N57 & .03 & $12 / 22$ & & 92 & UE25 WT-18 & $\mathbf{M}$ & & \\
\hline 21 & UE-25 UZN \#29 & $\mathbf{M}^{*}$ & & & 56 & USW UZ-N64 & $\mathbf{M}$ & & & 93 & USW H-5 & $\mathbf{M}^{*}$ & $12 / 22$ & \\
\hline 22 & UE-25 UZN \#30 & $\mathbf{M}^{*}$ & & & 58 & USW UZ-N65 & $\mathbf{M}^{*}$ & & & 94 & HRF Wedge & .04 & $12 / 22$ & \\
\hline
\end{tabular}


Table 2. Summary of precipitation data from the nonrecording gage network at Yucca Mountain, Nevada, for water years 1992 and 1993--Continued

\begin{tabular}{|c|c|c|c|c|c|c|c|c|c|c|c|c|c|c|}
\hline $\begin{array}{l}\text { Loca- } \\
\text { tlon } \\
\text { code }\end{array}$ & $\begin{array}{c}\text { Site } \\
\text { nomenclature }\end{array}$ & $\begin{array}{l}\text { Amount } \\
\text { (inches) }\end{array}$ & $\begin{array}{l}\text { Date } \\
\text { read }\end{array}$ & Notes & $\begin{array}{l}\text { Loca- } \\
\text { tion } \\
\text { code }\end{array}$ & $\begin{array}{c}\text { Site } \\
\text { nomenclature }\end{array}$ & $\begin{array}{l}\text { Amount } \\
\text { (inches) }\end{array}$ & $\begin{array}{l}\text { Date } \\
\text { read }\end{array}$ & Notes & $\begin{array}{l}\text { Loca- } \\
\text { tion } \\
\text { code }\end{array}$ & $\begin{array}{c}\text { Site } \\
\text { nomenclature }\end{array}$ & $\begin{array}{l}\text { Amount } \\
\text { (Inches) }\end{array}$ & $\begin{array}{l}\text { Date } \\
\text { read }\end{array}$ & Notes \\
\hline \multicolumn{15}{|c|}{ STORM OF DECEMBER 18, 1992-Continued } \\
\hline 23 & UE-25 UZN \#56 & $\mathbf{M}^{*}$ & & & 59 & USW UZ-N66 & $\mathbf{M}^{*}$ & & & 94 & HRF 4" Gage & .02 & $12 / 22$ & \\
\hline 24 & UE-25 UZN \#60 & .14 & $12 / 22$ & ** & 60 & USW UZ-N67 & $\mathbf{M}^{*}$ & & & 94 & HRF 8" Gage & .02 & $12 / 22$ & \\
\hline 25 & UE-25 UZN \#85 & $\mathbf{M}^{*}$ & & & 60 & N67 4" Gage & $\mathrm{M}^{*}$ & & & 95 & USW H-3 & $\mathrm{M}^{*}$ & & \\
\hline 26 & UE-25 UZN \#92 & $\mathbf{M}^{*}$ & & & 61 & USW UZ-N68 & $\mathbf{M}^{*}$ & & & 96 & Wea station & $\mathrm{M}^{*}$ & & \\
\hline 26 & N92 4" Gage & $\mathrm{M}^{*}$ & & & 62 & USW UZ-N69 & $\mathbf{M}^{*}$ & & & 97 & Wea station & M & & \\
\hline 27 & UE-25 UZN \#97 & .15 & $12 / 22$ & & 63 & USW UZ-N70 & .08 & $12 / 21$ & ** & 98 & Evaporation & .06 & $12 / 22$ & \\
\hline 28 & UE-29 UZN \#91 & $\mathbf{M}^{*}$ & & & 63 & N70 4" Gage & $\mathbf{M}$ & & & 99 & Wea station & $\mathrm{M}$ & & \\
\hline 28 & N91 4" Gage & $\mathrm{M}^{*}$ & & & 64 & USW UZ-N71 & $\mathbf{M}^{*}$ & & & 99 & Wea station & M & & \\
\hline 29 & USW UZ-N11 & $\mathbf{M}^{*}$ & & & 64 & N71 4" Gage & $\mathbf{M}$ & & & 100 & G-3 & $\mathbf{M}^{*}$ & & \\
\hline 29 & N11 4" Gage & $\mathrm{M}^{*}$ & & & 65 & USW UZ-N72 & $\mathbf{M}^{*}$ & & & 100 & G-3 8" Gage & $\mathbf{M}^{*}$ & & \\
\hline 30 & USW UZ-N15 & $\mathbf{M}^{*}$ & & & 66 & USW UZ-N73 & $\mathbf{M}^{*}$ & & & 101 & Wea station & M & & \\
\hline 31 & USW UZ-N16 & $\mathrm{M}^{*}$ & & & 67 & USW UZ-N74 & $\mathbf{M}^{*}$ & & & 101 & Wea station & $\mathbf{M}^{*}$ & & \\
\hline 32 & USW UZ-N17 & $\mathrm{M}^{*}$ & & & 68 & USW UZ-N75 & $\mathbf{M}^{*}$ & & & 102 & Wea station & $\mathrm{M}^{*}$ & & \\
\hline 33 & USW UZ-N24 & .09 & $12 / 21$ & ** & 68 & N75 4" Gage & $\mathbf{M}$ & & & 103 & Wea station & .01 & $12 / 21$ & \\
\hline 33 & N24 4" Gage & NI & & & 69 & USW UZ-N76 & $\mathbf{M}^{*}$ & & & 103 & Wea station & .01 & $12 / 21$ & \\
\hline 34 & USW UZ-N25 & .08 & $12 / 21$ & $* *$ & 70 & USW UZ-N77 & $\mathrm{M}^{*}$ & & & 104 & Fran Ridge & $\mathrm{M}^{*}$ & & \\
\hline 35 & USW UZ-N26 & .06 & $12 / 21$ & $* *$ & 70 & N77 4" Gage & Removed & & & 104 & Fran Ridge (8") & $\mathbf{M}^{*}$ & & \\
\hline 36 & USW UZ-N27 & 0 & $12 / 22$ & & 71 & USW UZ-N78 & $\mathrm{M}^{*}$ & & & 105 & Plug Hill & $\mathbf{M}^{*}$ & & \\
\hline 36 & N27 8" Gage & 0 & $12 / 22$ & & 72 & USW UZ-N79 & $\mathbf{M}^{*}$ & & & 106 & $4 \mathrm{JA}(8 ")$ & .05 & $12 / 21$ & \\
\hline 37 & USW UZ-N35 & .04 & $12 / 22$ & & 73 & USW UZ-N80 & $\mathrm{M}^{*}$ & & & 107 & Knothead Gap & $\mathrm{M}^{*}$ & & \\
\hline 38 & USW UZ-N36 & $\mathbf{M}^{*}$ & & & 73 & N80 4" Gage & $\mathrm{M}^{*}$ & & & 108 & Upper Forty Mile & $\mathbf{M}^{*}$ & & \\
\hline
\end{tabular}


Table 2. Summary of precipitation data from the nonrecording gage network at Yucca Mountain, Nevada, for water years 1992 and 1993--Continued

\begin{tabular}{|c|c|c|c|c|c|c|c|c|c|c|c|c|c|c|}
\hline $\begin{array}{l}\text { Loca- } \\
\text { tion } \\
\text { code }\end{array}$ & $\begin{array}{c}\text { Slte } \\
\text { nomenclature }\end{array}$ & $\begin{array}{l}\text { Amount } \\
\text { (inches) }\end{array}$ & $\begin{array}{l}\text { Date } \\
\text { read }\end{array}$ & Notes & $\begin{array}{l}\text { Loca- } \\
\text { tion } \\
\text { code }\end{array}$ & $\begin{array}{c}\text { Site } \\
\text { nomenclature }\end{array}$ & $\begin{array}{l}\text { Amount } \\
\text { (Inches) }\end{array}$ & $\begin{array}{l}\text { Date } \\
\text { read }\end{array}$ & Notes & $\begin{array}{l}\text { Loca- } \\
\text { tion } \\
\text { code }\end{array}$ & $\begin{array}{c}\text { Site } \\
\text { nomenclature }\end{array}$ & $\begin{array}{l}\text { Amount } \\
\text { (Inches) }\end{array}$ & $\begin{array}{l}\text { Date } \\
\text { read }\end{array}$ & Notes \\
\hline \multicolumn{15}{|c|}{ STORM OF DECEMBER 27-29, 1992} \\
\hline 1 & UE-25 UZN \#1 & .18 & $12 / 30$ & @ & 39 & USW UZ-N37 & .31 & $12 / 30$ & $* *$ & 74 & USW UZ-N81 & $\mathbf{M}^{*}$ & & \\
\hline 2 & UE-25 UZN \#2 & .34 & $12 / 30$ & ** & 39 & N37 4" Gage & .27 & $12 / 30$ & ** & 75 & USW UZ-N82 & $\mathbf{M}^{*}$ & & \\
\hline 3 & UE-25 UZN \#3 & .29 & $12 / 30$ & & 40 & USW UZ-N40 & .33 & $12 / 30$ & ** & 75 & N82 4" Gage & & & Removed \\
\hline 4 & UE-25 UZN \#4 & .35 & $12 / 30$ & @ & 40 & N40 4" Gage & NI & & & 76 & USW UZ-N83 & $\mathrm{M}^{*}$ & & \\
\hline 4 & N4 4" Gage & .29 & $12 / 30$ & & 41 & USW UZ-N41 & .39 & $12 / 30$ & $* *$ & 77 & USW UZ-N84 & $\mathbf{M}^{*}$ & & \\
\hline 5 & UE-25 UZN \#5 & 31 & $12 / 30$ & *** & 42 & USW UZ-N42 & .36 & $12 / 30$ & *** & 78 & USW UZ-N86 & $\mathrm{M}^{*}$ & & \\
\hline 6 & UE-25 UZN \#6 & .33 & $12 / 30$ & ** & 43 & USW UZ-N43 & .37 & $12 / 30$ & $* *$ & 79 & USW UZ-N87 & $\mathbf{M}^{*}$ & & \\
\hline 7 & UE-25 UZN \#7 & .35 & $12 / 30$ & $@$ & 43 & N43 4" Gage & NI & & & 80 & USW UZ-N88 & $\mathbf{M}^{*}$ & & \\
\hline 8 & UE-25 UZN \#8 & .40 & $12 / 30$ & ** & 44 & USW UZ-N44 & .38 & $12 / 30$ & $* *$ & 81 & USW UZ-N89 & $\mathbf{M}^{*}$ & & \\
\hline 9 & UE-25 UZN \#9 & .35 & $12 / 30$ & & 45 & USW UZ-N45 & .33 & $12 / 30$ & $* *$ & 82 & USW UZ-N90 & $\mathbf{M}^{*}$ & & \\
\hline 10 & UE-25 UZN \#10 & .35 & $12 / 30$ & $@$ & 46 & USW UZ-N46 & .36 & $12 / 30$ & $* *$ & 82 & N90 4" Gage & $\mathbf{M}^{*}$ & & \\
\hline 10 & N10 4" Gage & .30 & $12 / 30$ & & 47 & USW UZ-N47 & .46 & $12 / 30$ & $* *$ & 83 & USW UZ-N93 & .15 & & \\
\hline 11 & UE-25 UZN \#12 & .29 & $12 / 30$ & & 48 & USW UZ-N48 & .46 & $12 / 30$ & $* *$ & 84 & USW UZ-N94 & .25 & $12 / 28$ & $* *$ \\
\hline 12 & UE-25 UZN \#13 & .38 & $12 / 30$ & ** & 49 & USW UZ-N49 & $\mathbf{M}^{*}$ & & & 85 & USW UZ-N95 & .30 & $12 / 28$ & ** \\
\hline 13 & UE-25 UZN \#14 & .36 & $12 / 30$ & $* *$ & 50 & USW UZ-N50 & $\mathbf{M}^{*}$ & & & 86 & USW UZ-N96 & .37 & $12 / 28$ & $* *$ \\
\hline 14 & UE-25 UZN \#18 & .32 & $12 / 30$ & $* *$ & 50 & N50 4" Gage & NI & & & 87 & USW UZ-N98 & .41 & $12 / 28$ & $* *$ \\
\hline 15 & UE-25 UZN \#19 & .28 & $12 / 30$ & @ & 51 & USW UZ-N51 & $\mathbf{M}^{*}$ & & & 87 & N98 4" Gage & NI & & \\
\hline 16 & UE-25 UZN \#20 & .34 & $12 / 30$ & & 52 & USW UZ-N52 & $\mathbf{M}^{*}$ & & & 88 & USW UZ-13 & .27 & $12 / 28$ & \\
\hline 17 & UE-25 UZN \#21 & 0 & $12 / 30$ & & 53 & USW UZ-N53 & $\mathbf{M}^{*}$ & & & 88 & UZ-13 4" Gage & .15 & $12 / 28$ & \\
\hline 17 & N21 4" Gage & NI & & & 54 & USW UZ-N54 & $\mathrm{M}^{*}$ & & & 89 & USW GA-1 & .34 & $12 / 28$ & \\
\hline 18 & UE-25 UZN \#22 & .33 & $12 / 30$ & @ & 54 & N54 4" Gage & $\mathbf{M}^{*}$ & & & 90 & USW G-2 & .33 & $12 / 28$ & \\
\hline 19 & UE-25 UZN \#23 & $\mathbf{M}^{*}$ & & & 55 & USW UZ-N55 & $\mathbf{M}^{*}$ & & & 91 & UE25 WT-4 & .26 & $12 / 28$ & \\
\hline 20 & UE-25 UZN \#28 & .30 & $12 / 30$ & & 56 & USW UZ-N57 & .38 & $12 / 28$ & & 92 & UE25 WT-18 & .28 & $12 / 28$ & \\
\hline 21 & UE-25 UZN \#29 & $\mathrm{M}^{*}$ & & & 57 & USW UZ-N64 & .25 & $12 / 28$ & & 93 & USW H-5 & .15 & $12 / 28$ & $* *$ \\
\hline 22 & UE-25 UZN \#30 & $\mathrm{M}^{*}$ & & & 58 & USW UZ-N65 & .39 & $12 / 28$ & ** & 94 & HRF Wedge & .36 & $12 / 28$ & \\
\hline
\end{tabular}


Table 2. Summary of precipitation data from the nonrecording gage network at Yucca Mountain, Nevada, for water years 1992 and 1993 --Continued

\begin{tabular}{|c|c|c|c|c|c|c|c|c|c|c|c|c|c|c|}
\hline $\begin{array}{l}\text { Loca- } \\
\text { tion } \\
\text { code }\end{array}$ & $\begin{array}{c}\text { Sile } \\
\text { nomenclature }\end{array}$ & $\begin{array}{l}\text { Amount } \\
\text { (inches) }\end{array}$ & $\begin{array}{l}\text { Date } \\
\text { read }\end{array}$ & Notes & $\begin{array}{l}\text { Loca- } \\
\text { tion } \\
\text { code }\end{array}$ & $\begin{array}{c}\text { Site } \\
\text { nomenclature }\end{array}$ & $\begin{array}{l}\text { Amount } \\
\text { (inches) }\end{array}$ & $\begin{array}{l}\text { Date } \\
\text { read }\end{array}$ & Notes & $\begin{array}{l}\text { Loca- } \\
\text { tion } \\
\text { code }\end{array}$ & $\begin{array}{c}\text { Sile } \\
\text { nomenclature }\end{array}$ & $\begin{array}{l}\text { Amount } \\
\text { (inches) }\end{array}$ & $\begin{array}{l}\text { Date } \\
\text { read }\end{array}$ & Notes \\
\hline \multicolumn{15}{|c|}{ STORM OF DECEMBER 27-29, 1992-Continued } \\
\hline 23 & UE-25 UZN \#56 & $\mathbf{M}^{*}$ & & & 59 & USW UZ-N66 & .34 & $12 / 28$ & ** & 94 & HRF 4" Gage & .38 & $12 / 28$ & \\
\hline 24 & UE-25 UZN \#60 & $\mathbf{M}^{*}$ & $@$ & & 60 & USW UZ-N67 & .38 & $12 / 28$ & $* *$ & 94 & HRF 8" Gage & .37 & $12 / 28$ & \\
\hline 25 & UE-25 UZN \#85 & .33 & $12 / 28$ & ** & 60 & N67 4" Gage & .38 & $12 / 28$ & ** & 95 & USW H-3 & .04 & $12 / 28$ & \\
\hline 26 & UE-25 UZN \#92 & .33 & $12 / 29$ & ** & 61 & USW UZ-N68 & .40 & $12 / 28$ & ** & 96 & Wea station & .17 & $12 / 28$ & \\
\hline 26 & N92 4" Gage & .41 & $12 / 29$ & $* *$ & 62 & USW UZ-N69 & .39 & $12 / 28$ & $* *$ & 97 & Wea station & $\mathbf{M}$ & & \\
\hline 27 & UE-25 UZN \#97 & .33 & $12 / 30$ & @ & 63 & USW UZ-N70 & .36 & $12 / 30$ & & 98 & Evaporation & .31 & $12 / 28$ & \\
\hline 28 & UE-29 UZN \#91 & .42 & $12 / 29$ & $* *$ & 63 & N70 4" Gage & .31 & $12 / 30$ & & 99 & Wea station & .12 & $12 / 28$ & \\
\hline 28 & N91 4" Gage & .48 & $12 / 29$ & ** & 64 & USW UZ-N71 & .15 & $12 / 30$ & $* *$ & 99 & Wea station & .13 & $12 / 28$ & \\
\hline 29 & USW UZ-N11 & .38 & $12 / 29$ & $* *$ & 64 & N71 4" Gage & $\mathbf{M}$ & & & 100 & G-3 & .37 & $12 / 28$ & ** \\
\hline 29 & N11 4" Gage & .33 & $12 / 29$ & $* *$ & 65 & USW UZ-N72 & .17 & $12 / 28$ & $* *$ & 100 & G-3 8" Gage & .24 & $12 / 28$ & \\
\hline 30 & USW UZ-N15 & .34 & $12 / 29$ & $* *$ & 66 & USW UZ-N73 & .22 & $12 / 28$ & ** & 101 & Wea station & .23 & $12 / 30$ & \\
\hline 31 & USW UZ-N16 & .34 & $12 / 29$ & ** & 67 & USW UZ-N74 & .21 & $12 / 28$ & ** & 101 & Wea station & .39 & $12 / 30$ & \\
\hline 32 & USW UZ-N17 & .33 & $12 / 29$ & $* *$ & 68 & USW UZ-N75 & .20 & $12 / 28$ & $* *$ & 102 & Wea station & .30 & $12 / 30$ & \\
\hline 33 & USW UZ-N24 & .31 & $12 / 30$ & & 68 & N75 4" Gage & M & & & 103 & Wea station & .40 & $12 / 30$ & \\
\hline 33 & N24 4" Gage & $\mathrm{NI}$ & & & 69 & USW UZ-N76 & 1.47 & $12 / 28$ & ** & 103 & Wea station & .37 & $12 / 30$ & \\
\hline 34 & USW UZ-N25 & .33 & $12 / 30$ & & 70 & USW UZ-N77 & $\mathrm{M}^{*}$ & & & 104 & Fran Ridge & $\mathrm{M}^{*}$ & & \\
\hline 35 & USW UZ-N26 & .34 & $12 / 30$ & & 70 & N77 4" Gage & Removed & & & 104 & Fran Ridge (8") & $\mathrm{M}^{*}$ & & \\
\hline 36 & USW UZ-N27 & .33 & $12 / 28$ & & 71 & USW UZ-N78 & $\mathbf{M}^{*}$ & & & 105 & Plug Hill & $\mathrm{M}^{*}$ & & \\
\hline 36 & N27 8" Gage & .28 & $12 / 28$ & & 72 & USW UZ-N79 & $\mathrm{M}^{*}$ & & & 106 & 4JA (8") & .36 & $12 / 28$ & \\
\hline 37 & USW UZ-N35 & $\mathrm{M}^{*}$ & & & 73 & USW UZ-N80 & $\mathrm{M}^{*}$ & & & 107 & Knothead Gap & $\mathrm{M}^{*}$ & & \\
\hline 38 & USW UZ-N36 & .32 & $12 / 28$ & $* *$ & 73 & N80 4" Gage & $\mathrm{M}^{*}$ & & & 108 & Upper Forty Mile & .46 & $12 / 28$ & ** \\
\hline
\end{tabular}


Table 2. Summary of precipitation data from the nonrecording gage network at Yucca Mountain, Nevada, for water years 1992 and $1993--$ Continued

\begin{tabular}{|c|c|c|c|c|c|c|c|c|c|c|c|c|c|c|}
\hline $\begin{array}{l}\text { Loca- } \\
\text { Ilon } \\
\text { code }\end{array}$ & $\begin{array}{c}\text { Site } \\
\text { nomenclature }\end{array}$ & $\begin{array}{l}\text { Amount } \\
\text { (inches) }\end{array}$ & $\begin{array}{l}\text { Date } \\
\text { read }\end{array}$ & Notes & $\begin{array}{l}\text { Loca- } \\
\text { tion } \\
\text { code }\end{array}$ & $\begin{array}{c}\text { Sile } \\
\text { nomenclature }\end{array}$ & $\begin{array}{l}\text { Amount } \\
\text { (inches) }\end{array}$ & $\begin{array}{l}\text { Date } \\
\text { read }\end{array}$ & Notes & $\begin{array}{l}\text { Loca- } \\
\text { tion } \\
\text { code }\end{array}$ & $\begin{array}{c}\text { Site } \\
\text { nomenclature }\end{array}$ & $\begin{array}{l}\text { Amount } \\
\text { (Inches) }\end{array}$ & $\begin{array}{l}\text { Date } \\
\text { read }\end{array}$ & Notes \\
\hline \multicolumn{15}{|c|}{ STORM OF JANUARY 2, 1993} \\
\hline 1 & UE-25 UZN \#1 & .02 & $1 / 5$ & FZN & 38 & USW UZ-N36 & M & & & 73 & N80 4" Gage & $\mathbf{M}^{*}$ & & \\
\hline 2 & UE-25 UZN \#2 & .03 & $1 / 5$ & $\mathrm{FZN}$ & 39 & USW UZ-N37 & .02 & $1 / 5$ & & 74 & USW UZ-N81 & $\mathrm{M}^{*}$ & & \\
\hline 3 & UE-25 UZN \#3 & .01 & $1 / 5$ & FZN & 39 & N37 4" Gage & .02 & $1 / 5$ & & 75 & USW UZ-N82 & $\mathrm{M}^{*}$ & & \\
\hline 4 & UE-25 UZN \#4 & .02 & $1 / 5$ & FZN & 40 & USW UZ-N40 & .01 & $1 / 5$ & & 76 & USW UZ-N83 & $\mathrm{M}^{*}$ & & \\
\hline 4 & N4 4" Gage & M & & & 40 & N40 4" Gage & NI & & & 77 & USW UZ-N84 & $\mathrm{M}^{*}$ & & \\
\hline 5 & UE-25 UZN \#5 & .03 & $1 / 5$ & FZN & 41 & USW UZ-N41 & $\mathbf{M}^{*}$ & & & 78 & USW UZ-N86 & $\mathrm{M}^{*}$ & & \\
\hline 6 & UE-25 UZN \#6 & .02 & $1 / 5$ & FZN & 42 & USW UZ-N42 & 0 & $1 / 5$ & & 79 & USW UZ-N87 & $\mathrm{M}^{*}$ & & \\
\hline 7 & UE-25 UZN \#7 & .02 & $1 / 5$ & FZN & 43 & USW UZ-N43 & .02 & $1 / 5$ & & 80 & USW UZ-N88 & $\mathbf{M}^{*}$ & & \\
\hline 8 & UE-25 UZN \#8 & .03 & $1 / 5$ & FZN & 43 & N43 4" Gage & NI & & & 81 & USW UZ-N89 & $\mathrm{M}^{*}$ & & \\
\hline 9 & UE-25 UZN \#9 & .03 & $1 / 5$ & FZN & 44 & USW UZ-N44 & 0 & $1 / 5$ & & 82 & USW UZ-N90 & $\mathbf{M}^{*}$ & & \\
\hline 10 & UE-25 UZN \#10 & .02 & $1 / 5$ & FZN & 45 & USW UZ-N45 & .01 & $1 / 5$ & & 82 & N90 4" Gage & $\mathrm{M}^{*}$ & & \\
\hline 10 & N10 4" Gage & M & & & 46 & USW UZ-N46 & 0 & $1 / 5$ & & 83 & USW UZ-N93 & $\mathrm{M}^{*}$ & & \\
\hline 11 & UE-25 UZN \#12 & .01 & $1 / 5$ & FZN & 47 & USW UZ-N47 & .01 & $1 / 5$ & & 84 & USW UZ-N94 & $\mathrm{M}^{*}$ & & \\
\hline 12 & UE-25 UZN \#13 & .03 & $1 / 5$ & & 48 & USW UZ-N48 & $\mathrm{M}^{*}$ & & & 85 & USW UZ-N95 & $\mathrm{M}^{*}$ & & \\
\hline 13 & UE-25 UZN \#14 & .02 & $1 / 5$ & FZN & 49 & USW UZ-N49 & $\mathbf{M}^{*}$ & & & 86 & USW UZ-N96 & $\mathbf{M}^{*}$ & $1 / 5$ & \\
\hline 14 & UE-25 UZN \#18 & .03 & $1 / 5$ & FZN & 50 & USW UZ-N50 & $\mathbf{M}^{*}$ & & & 87 & USW UZ-N98 & 0 & & \\
\hline 15 & UE-25 UZN \#19 & 0 & & & 50 & N50 4" Gage & NI & & & 87 & N98 4" Gage & NI & & \\
\hline 16 & UE-25 UZN \#20 & .01 & $1 / 5$ & FZN & 51 & USW UZ-N51 & $\mathbf{M}^{*}$ & & & 88 & UZ-13 4" Gage & $\mathbf{M}$ & & \\
\hline 17 & UE-25 UZN \#21 & 0 & $1 / 5$ & FZN & 52 & USW UZ-N52 & $\mathbf{M}^{*}$ & & & 89 & USW UZ-13 & $\mathrm{M}^{*}$ & & \\
\hline 17 & N21 4" Gage & $\mathrm{NI}$ & & & 53 & USW UZ-N53 & .24 & $1 / 5$ & & 89 & USW GA-1 & $\mathbf{M}$ & & \\
\hline 18 & UE-25 UZN \#22 & .03 & $1 / 5$ & FZN & 54 & USW UZ-N54 & .29 & $1 / 5$ & & 90 & USW G-2 & $\mathbf{M}$ & $1 / 5$ & \\
\hline 19 & UE-25 UZN \#23 & .34 & $* *$ & & 54 & N54 4" Gage & .31 & $1 / 5$ & & 91 & UE25 WT-4 & .01 & & \\
\hline 20 & UE-25 UZN \#28 & .01 & $1 / 5$ & FZN & 55 & USW UZ-N55 & .32 & $1 / 5$ & & 92 & UE25 WT-18 & $\mathbf{M}$ & & \\
\hline 21 & UE-25 UZN \#29 & .46 & $1 / 5$ & $* *$ & 56 & USW UZ-N57 & .02 & $1 / 5$ & & 93 & USW H-5 & $\mathbf{M}^{*}$ & $1 / 4$ & \\
\hline 22 & UE-25 UZN \#30 & .46 & $1 / 5$ & ** & 57 & USW UZ-N64 & $\mathbf{M}$ & & & 94 & HRF Wedge & .04 & $1 / 4$ & \\
\hline
\end{tabular}




\begin{tabular}{|c|c|c|c|c|c|c|c|c|c|c|c|c|c|c|}
\hline & & $\varepsilon 0^{\circ}$ & 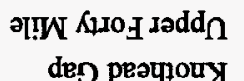 & $\begin{array}{l}801 \\
\angle 01\end{array}$ & & & & & & & & & & \\
\hline \multirow{20}{*}{ ** } & S/I & $t 0^{\circ}$ & & $\angle 0 I$ & & & $* \mathrm{~W}$ & 08N-Zn MSn & $\varepsilon L$ & & $\boldsymbol{s} / \mathrm{l}$ & $\tau \varepsilon^{\cdot}$ & รЕN-Zn MSก & $L \varepsilon$ \\
\hline & $S / I$ & $+0^{\circ}$ & (u8) vIt & 901 & & & $* \mathrm{~W}$ & 6LN-Zn MSn & $z L$ & & & $* \mathbb{W}$ & วฮีषD „8 LZN & $9 \varepsilon$ \\
\hline & $t / \mathbf{I}$ & $* \mathrm{~W}$ & II!H ฮีn!d & soI & & & $* \mathrm{~W}$ & $8 L N-Z \cap M S \cap$ & IL & & & $* \mathrm{~W}$ & $\angle Z N=Z n M S \cap$ & $9 \mathfrak{\varepsilon}$ \\
\hline & & $* \mathrm{~N}$ & 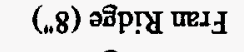 & toI & & & $* \mathrm{~W}$ & $\angle L N-Z \cap M S \cap$ & $O L$ & & & $* \mathbf{W}$ & 9ZN-Zn MSO & SE \\
\hline & & $* \mathrm{~N}$ & 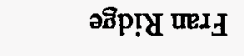 & tol & & & $* \mathrm{~W}$ & 9LN-Zn MSn & 69 & & & $* \mathrm{~N}$ & $\varsigma Z N-Z \cap M S \cap$ & $t \varepsilon$ \\
\hline & & to & 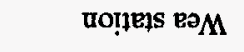 & $\varepsilon 01$ & & & $* \mathbf{W}$ & วธี้อ „ „ & 89 & & & IN & วชิED „t t乙N & $\varepsilon \varepsilon$ \\
\hline & $s / I$ & 0 & 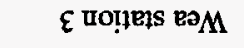 & $\varepsilon 01$ & & & $* \mathrm{~N}$ & $S \angle N-Z \cap M S \cap$ & 89 & & s/L & 0 & $t_{Z N-Z n} M S \cap$ & $\varepsilon \mathfrak{\varepsilon}$ \\
\hline & $s / I$ & $* \mathrm{~W}$ & 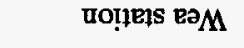 & $z 01$ & & & $* \mathbf{W}$ & $t \angle N-Z \cap M S \cap$ & $\angle 9$ & & & $* \mathrm{~N}$ & LIN-Zn MSO & $\tau \varepsilon$ \\
\hline & NZJ & E0 & 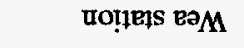 & I0I & & & $* W$ & $\varepsilon\llcorner N-Z \cap M S \cap$ & 99 & & & $* \mathrm{~W}$ & $91 N^{-Z} \mathrm{Zn}$ MSO & $\mathfrak{I} \mathfrak{\varepsilon}$ \\
\hline & $s / I$ & 0 & पO!ฺES EอM & I0I & & & $* \mathrm{~N}$ & ZLN-Zn MSR & s9 & & & $* \mathrm{~N}$ & SIN-Zn MSO & $o \varepsilon$ \\
\hline & $S / I$ & $* \mathbf{W}$ & 28ిย & 001 & & & $* \mathbf{W}$ & 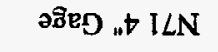 & 59 & & & $* \mathrm{~W}$ & $28 \mathrm{ED}$. & $6 \tau$ \\
\hline & & $* \mathrm{~N}$ & $\varepsilon-\mathcal{D}$ & 001 & & & $* \mathrm{~W}$ & ILN-Zn MSn & $t 9$ & & $s / l$ & $* \mathrm{~W}$ & IIN-Zn MSO & 62 \\
\hline & & w & uọ!les вәM & 66 & & & $* \mathrm{~W}$ & 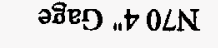 & $\varepsilon 9$ & & $s / I$ & $\mathrm{Or}^{\circ}$ & ว8ైD „t I6N & 82 \\
\hline & & w & toinels eep & 66 & & & $* \mathrm{~W}$ & $0\llcorner\mathrm{~N}-\mathrm{Zn} M \mathrm{MSn}$ & ⿷9 & & $s / l$ & $6 \mathrm{I}^{\circ}$ & I6\# NZn 6Z-30 & 82 \\
\hline & & $\varepsilon 0^{\circ}$ & uo!̣EJode $\mathrm{G}$ & 86 & & 9/t & $20^{\circ}$ & 69N-Zn MSn & 29 & & $s / t$ & $\mathfrak{E} 0^{\circ}$ & 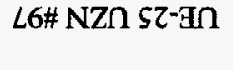 & $\angle z$ \\
\hline & $\mathbf{t} / \mathbf{l}$ & $\mathbf{w}$ & uọ!p?s вәМ & $\angle 6$ & & 9/I & $\mathfrak{\varepsilon} 0^{\circ}$ & 89N-Zn MSn & I9 & & s/I & $\mathfrak{E} 0^{\circ}$ & ว8ิ8D „t Z6N & $9 z$ \\
\hline & & $* \mathbf{N}$ & uо!̣घ!s вәМ & 96 & & 9/1 & $\mathfrak{1 0}$ & อ8ిש „.t L9N & 09 & NZI & $S / \mathrm{I}$ & $\mathrm{zI^{ \circ }}$ & 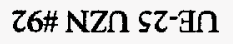 & $9 z$ \\
\hline & & $* \mathrm{~N}$ & $\varepsilon-H M S \Omega$ & \$6 & & 9/I & $\varepsilon 0^{\circ}$ & L9N-Zn MSก & 09 & & $s / 1$ & $10^{\circ}$ & 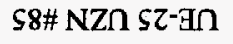 & 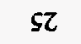 \\
\hline & & $\angle 0^{\circ}$ & 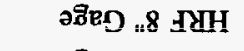 & $t 6$ & & & $=\mathbb{N}$ & 99N-Zn MSn & $6 s$ & $* *$ & $\varsigma / I$ & $91^{\circ}$ & 00\# NZO SZ-B⿵ & $\mapsto z$ \\
\hline & $t / \mathrm{l}$ & $50^{\circ}$ & 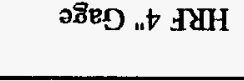 & t6 & & $1000-\varepsilon$ & 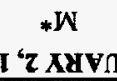 & $\begin{array}{l}\text { S9N-Zn MSn } \\
\text { vP AO WyOLS }\end{array}$ & $8 S$ & $* *$ & $s / l$ & $\angle E^{\circ}$ & 9S\# NZn ऽZ-تß & $\varepsilon z$ \\
\hline SelON & $\begin{array}{l}\text { pead } \\
\text { elead }\end{array}$ & $\begin{array}{l}\text { (seypu!) } \\
\text { junowy }\end{array}$ & $\begin{array}{l}\text { enniejouowou } \\
\text { ells }\end{array}$ & $\begin{array}{c}\text { opos } \\
\text { uoll } \\
-80007\end{array}$ & selon & $\begin{array}{l}\text { peed } \\
\text { oleo }\end{array}$ & $\begin{array}{l}\text { (seyou!) } \\
\text { lunowit }\end{array}$ & $\begin{array}{l}\text { exntepouewou } \\
\text { eyIS }\end{array}$ & $\begin{array}{l}\text { opos } \\
\text { u०|l } \\
-8007\end{array}$ & selon & $\begin{array}{l}\text { peed } \\
\text { opea }\end{array}$ & $\begin{array}{l}\text { (seypu!) } \\
\text { lunoury }\end{array}$ & $\begin{array}{l}\text { edniepouøurou } \\
\text { elIS }\end{array}$ & $\begin{array}{c}\text { opos } \\
\text { UO! } \\
-8007\end{array}$ \\
\hline
\end{tabular}


Table 2. Summary of precipitation data from the nonrecording gage network at Yucca Mountain, Nevada, for water years 1992 and 1993--Continued

\begin{tabular}{|c|c|c|c|c|c|c|c|c|c|c|c|c|c|c|}
\hline $\begin{array}{l}\text { Loca- } \\
\text { tion } \\
\text { code }\end{array}$ & $\begin{array}{c}\text { Site } \\
\text { nomenelature }\end{array}$ & $\begin{array}{l}\text { Amount } \\
\text { (inches) }\end{array}$ & $\begin{array}{l}\text { Date } \\
\text { read }\end{array}$ & Notes & $\begin{array}{l}\text { Loca- } \\
\text { tion } \\
\text { code }\end{array}$ & $\begin{array}{c}\text { Site } \\
\text { nomenclature }\end{array}$ & $\begin{array}{l}\text { Amount } \\
\text { (inches) }\end{array}$ & $\begin{array}{l}\text { Date } \\
\text { read }\end{array}$ & Notes & $\begin{array}{l}\text { Loca- } \\
\text { tion } \\
\text { code }\end{array}$ & $\begin{array}{c}\text { Site } \\
\text { nomenelature }\end{array}$ & $\begin{array}{l}\text { Amount } \\
\text { (inches) }\end{array}$ & $\begin{array}{l}\text { Date } \\
\text { read }\end{array}$ & Notes \\
\hline \multicolumn{15}{|c|}{ STORM OF JANUARY 6-7, 1993} \\
\hline 1 & UE-25 UZN \#1 & .50 & $1 / 8$ & & 38 & USW UZ-N36 & .47 & $1 / 8$ & & 73 & N80 4" Gage & 1.05 & $1 / 8$ & ** \\
\hline 2 & UE-25 UZN \#2 & .53 & $1 / 8$ & & 39 & USW UZ-N37 & .51 & $1 / 8$ & & 74 & USW UZ-N81 & .89 & $1 / 8$ & $* *$ \\
\hline 3 & UE-25 UZN \#3 & .47 & $1 / 8$ & & 39 & N37 4" Gage & .51 & $1 / 8$ & & 75 & USW UZ-N82 & .77 & $1 / 8$ & $* *$ \\
\hline 4 & UE-25 UZN $\# 4$ & .52 & $1 / 8$ & & 40 & USW UZ-N40 & .48 & $1 / 8$ & & 76 & USW UZ-N83 & .93 & $1 / 8$ & ** \\
\hline 4 & N4 4" Gage & .13 & $1 / 8$ & EVAP & 40 & N40 4" Gage & NI & & & 77 & USW UZ-N84 & .74 & & \\
\hline 5 & UE-25 UZN \#5 & .45 & $1 / 8$ & & 41 & USW UZ-N41 & .53 & $1 / 8$ & ** & 78 & USW UZ-N86 & .95 & $1 / 8$ & \\
\hline 6 & UE-25 UZN \#6 & .46 & $1 / 8$ & & 42 & USW UZ-N42 & .49 & $1 / 8$ & & 79 & USW UZ-N87 & .85 & $1 / 8$ & ** \\
\hline 7 & UE-25 UZN \#7 & .53 & $1 / 8$ & & 43 & USW UZ-N43 & .52 & $1 / 8$ & & 80 & USW UZ-N88 & .97 & $1 / 8$ & *** \\
\hline 8 & UE-25 UZN \#8 & .44 & $1 / 8$ & & 43 & N43 4" Gage & NI & & & 81 & USW UZ-N89 & .81 & $1 / 8$ & ** \\
\hline 9 & UE-25 UZN \#9 & .51 & $1 / 8$ & & 44 & USW UZ-N44 & .54 & $1 / 8$ & & 82 & USW UZ-N90 & .75 & $1 / 8$ & ** \\
\hline 10 & UE-25 UZN \#10 & .52 & $1 / 8$ & & 45 & USW UZ-N45 & .49 & $1 / 8$ & & 82 & N90 4" Gage & .81 & $1 / 8$ & ** \\
\hline 10 & N10 4" Gage & .12 & $1 / 8$ & EVAP & 46 & USW UZ-N46 & .49 & $1 / 8$ & & 83 & USW UZ-N93 & & & \\
\hline 11 & UE-25 UZN \#12 & .51 & $1 / 8$ & & 47 & USW UZ-N47 & .48 & $1 / 8$ & & 84 & USW UZ-N94 & .45 & $1 / 8$ & ** \\
\hline 12 & UE-25 UZN \#13 & .55 & $1 / 8$ & & 48 & USW UZ-N48 & .96 & $1 / 8$ & $* *$ & 85 & USW UZ-N95 & .34 & $1 / 8$ & \\
\hline 13 & UE-25 UZN \#14 & .48 & $1 / 8$ & & 49 & USW UZ-N49 & 1.00 & $1 / 8$ & $* *$ & 86 & USW UZ-N96 & .45 & $1 / 8$ & \\
\hline 14 & UE-25 UZN \#18 & .42 & $1 / 8$ & & 50 & USW UZ-N50 & .95 & $1 / 8$ & $m *$ & 87 & USW UZ-N98 & .49 & $1 / 8$ & \\
\hline 15 & UE-25 UZN \#19 & .15 & $1 / 8$ & EVAP & 50 & N50 4" Gage & $\mathrm{NI}$ & & & 87 & N98 4" Gage & NI & & \\
\hline 16 & UE-25 UZN \#20 & .51 & $1 / 8$ & & 51 & USW UZ-N51 & .89 & $1 / 8$ & $* *$ & 88 & USW UZ-13 & .35 & $1 / 8$ & \\
\hline 17 & UE-25 UZN \#21 & .51 & $1 / 8$ & & 52 & USW UZ-N52 & .91 & $1 / 8$ & ** & 88 & UZ-13 4" Gage & .31 & $1 / 8$ & \\
\hline 17 & N21 4" Gage & NI & & & 53 & USW UZ-N53 & .61 & $1 / 8$ & & 89 & USW GA-1 & .42 & $1 / 8$ & \\
\hline 18 & UE-25 UZN \#22 & .55 & $1 / 8$ & & 54 & USW UZ-N54 & .59 & $1 / 8$ & & 90 & USW G.2 & .44 & $1 / 8$ & \\
\hline 19 & UE-25 UZN \#23 & .52 & $1 / 8$ & & 54 & N54 4" Gage & .55 & $1 / 8$ & & 91 & UE25 WT-4 & .45 & $1 / 8$ & \\
\hline 20 & UE-25 UZN \#28 & .54 & $1 / 8$ & & 55 & USW UZ-N55 & .51 & $1 / 8$ & & 92 & UE25 WT-18 & .44 & $1 / 8$ & \\
\hline 21 & UE-25 UZN \#29 & $\mathbf{M}^{*}$ & & & 56 & USW UZ-N57 & .57 & $1 / 8$ & & 93 & USW H-5 & .35 & $1 / 8$ & $* *$ \\
\hline 22 & UE-25 UZN \#30 & $\mathbf{M}^{*}$ & & & 57 & USW UZ-N64 & $\mathbf{M}$ & & & 94 & HRF Wedge & $M^{*}$ & & \\
\hline
\end{tabular}


N Table 2. Summary of precipitation data from the nonrecording gage network at Yucca Mountain, Nevada, for water years 1992 and $1993-$-Continued

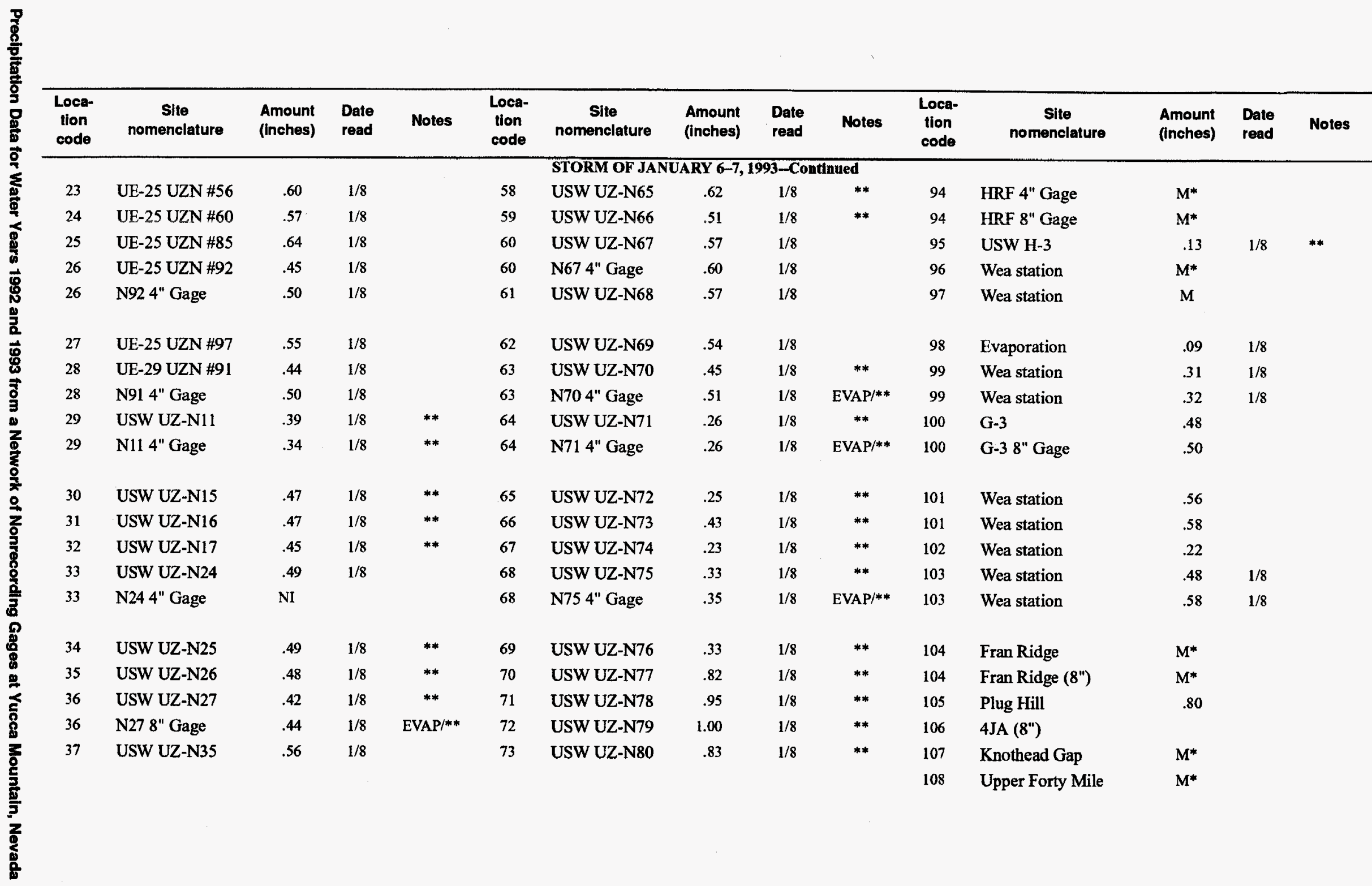


Table 2. Summary of precipitation data from the nonrecording gage network at Yucca Mountain, Nevada, for water years 1992 and 1993--Continued

\begin{tabular}{|c|c|c|c|c|c|c|c|c|c|c|c|c|c|c|}
\hline $\begin{array}{l}\text { Loca- } \\
\text { tion } \\
\text { code }\end{array}$ & $\begin{array}{c}\text { Site } \\
\text { nomenclature }\end{array}$ & $\begin{array}{l}\text { Amount } \\
\text { (inches) }\end{array}$ & $\begin{array}{l}\text { Date } \\
\text { read }\end{array}$ & Notes & $\begin{array}{l}\text { Loce- } \\
\text { tlon } \\
\text { code }\end{array}$ & $\begin{array}{c}\text { Site } \\
\text { nomenclature }\end{array}$ & $\begin{array}{l}\text { Amount } \\
\text { (inches) }\end{array}$ & $\begin{array}{l}\text { Date } \\
\text { read }\end{array}$ & Notes & $\begin{array}{l}\text { Loca- } \\
\text { tlon } \\
\text { code }\end{array}$ & $\begin{array}{c}\text { Site } \\
\text { nomenclature }\end{array}$ & $\begin{array}{l}\text { Amount } \\
\text { (inches) }\end{array}$ & $\begin{array}{l}\text { Date } \\
\text { read }\end{array}$ & Notes \\
\hline \multicolumn{15}{|c|}{ STORM OF JANUARY 12-14, 1993} \\
\hline 1 & UE-25 UZN \#1 & 1.04 & $1 / 14$ & & 38 & USW UZ-N36 & .90 & $1 / 14$ & & 73 & N80 4" Gage & $\mathbf{M}^{*}$ & & \\
\hline 2 & UE-25 UZN \#2 & 1.08 & $1 / 14$ & & 39 & USW UZ-N37 & .97 & $1 / 14$ & & 74 & USW UZ-N81 & $\mathrm{M}^{*}$ & & \\
\hline 3 & UE-25 UZN \#3 & 98 & $1 / 14$ & & 39 & N37 4" Gage & 1.05 & $1 / 14$ & & 75 & USW UZ-N82 & $\mathbf{M}^{*}$ & & \\
\hline 4 & UE-25 UZN \#4 & 1.05 & $1 / 14$ & & 40 & USW UZ-N40 & .93 & $1 / 14$ & & 76 & USW UZ-N83 & $\mathbf{M}^{*}$ & & \\
\hline 4 & N4 4" Gage & .99 & $1 / 14$ & & 40 & N40 4" Gage & NI & & & 77 & USW UZ-N84 & $\mathbf{M}^{*}$ & & \\
\hline 5 & UE-25 UZN \#5 & .98 & $1 / 14$ & & 41 & USW UZ-N41 & .80 & $1 / 14$ & & 78 & USW UZ-N86 & $\mathbf{M}^{*}$ & & \\
\hline 6 & UE-25 UZN \#6 & .98 & $1 / 14$ & & 42 & USW UZ-N42 & .98 & $1 / 14$ & & 79 & USW UZ-N87 & $\mathbf{M}^{*}$ & & \\
\hline 7 & UE-25 UZN \#7 & 1.03 & $1 / 14$ & & 43 & USW UZ-N43 & .97 & $1 / 14$ & & 80 & USW UZ-N88 & $\mathbf{M}^{*}$ & & \\
\hline 8 & UE-25 UZN \#8 & .98 & $1 / 14$ & & 43 & N43 4" Gage & NI & & & 81 & USW UZ-N89 & $\mathrm{M}^{*}$ & & \\
\hline 9 & UE-25 UZN \#9 & 1.06 & $1 / 14$ & & 44 & USW UZ-N44 & .97 & $1 / 14$ & & 82 & USW UZ-N90 & $\mathrm{M}^{*}$ & & \\
\hline 10 & UE-25 UZN \#10 & 1.02 & $1 / 14$ & & 45 & USW UZ-N45 & .84 & $1 / 14$ & & 82 & N90 4" Gage & $\mathbf{M}^{*}$ & & \\
\hline 10 & N10 4" Gage & 1.00 & $1 / 14$ & & 46 & USW UZ-N46 & .82 & $1 / 14$ & & 83 & USW UZ-N93 & .71 & & \\
\hline 11 & UE-25 UZN\#12 & 1.00 & $1 / 14$ & & 47 & USW UZ-N47 & .88 & $1 / 14$ & & 84 & USW UZ-N94 & .65 & $1 / 15$ & \\
\hline 12 & UE-25 UZN \#13 & 1.07 & $1 / 14$ & & 48 & USW UZ-N48 & 1.00 & $1 / 14$ & & 85 & USW UZ-N95 & .61 & $1 / 15$ & \\
\hline 13 & UE-25 UZN \#14 & .93 & $1 / 14$ & & 49 & USW UZ-N49 & .83 & $1 / 14$ & & 86 & USW UZ-N96 & .65 & $1 / 15$ & \\
\hline & & & & & & & & & & & & & $1 / 14$ & \\
\hline 14 & UE-25 UZN \#18 & .95 & $1 / 14$ & & 50 & USW UZ-N50 & .90 & $1 / 14$ & & 87 & USW UZ-N98 & .95 & $1 / 15$ & \\
\hline 15 & UE-25 UZN \#19 & 1.01 & $1 / 14$ & & 50 & N50 4" Gage & NI & & & 87 & N98 4" Gage & NI & & \\
\hline 16 & UE-25 UZN \#20 & 1.01 & $1 / 14$ & & 51 & USW UZ-N51 & .97 & $1 / 14$ & & 88 & USW UZ-13 & .88 & $1 / 14$ & \\
\hline 17 & UE-25 UZN \#21 & 1.06 & $1 / 14$ & & 52 & USW UZ-N52 & .91 & $1 / 14$ & & 88 & UZ-13 4" Gage & .54 & $1 / 14$ & \\
\hline 17 & N21 4" Gage & NI & & & 53 & USW UZ-N53 & .94 & $1 / 14$ & & 89 & USW GA-1 & .70 & $1 / 14$ & \\
\hline 18 & UE-25 UZN \#22 & 1.04 & $1 / 14$ & & 54 & USW UZ-N54 & .97 & $1 / 14$ & & 90 & USW G-2 & .76 & $1 / 14$ & \\
\hline 19 & UE-25 UZN \#23 & .99 & $1 / 14$ & & 54 & N54 4" Gage & 1.00 & & EVAP & 91 & UE25 WT-4 & .88 & $1 / 14$ & \\
\hline 20 & UE-25 UZN \#28 & .93 & $1 / 14$ & & 55 & USW UZ-N55 & .93 & & EVAP & 92 & UE25 WT-18 & .76 & $1 / 14$ & \\
\hline 21 & UE-25 UZN \#29 & 1.42 & $1 / 14$ & $* *$ & 56 & USW UZ-N57 & .93 & $1 / 14$ & & 93 & USW H-5 & .55 & & \\
\hline 22 & UE-25 UZN \#30 & 1.49 & $1 / 14$ & ** & 57 & USW UZ-N64 & .70 & & EVAP & 94 & HRF Wedge & .91 & $1 / 14$ & ** \\
\hline
\end{tabular}




\begin{tabular}{|c|c|c|c|c|c|c|c|c|c|c|c|c|c|c|}
\hline \multirow[t]{2}{*}{$* *$} & $\nabla \mathrm{I} / \mathrm{I}$ & 961 & 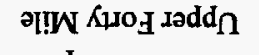 & 801 & & & & & & & & & & \\
\hline & $\boldsymbol{t} \mathbf{I} / \mathrm{I}$ & +6.1 & dep praqlot: & LOI & & & $* W$ & 08N-Zn MSח & $\varepsilon L$ & & $t I / I$ & $\angle 0^{\circ} I$ & SEN-Zn MSก & $L \mathcal{E}$ \\
\hline \multirow[t]{2}{*}{ *** } & $t I / I$ & $\angle t^{\circ}$ & $(.8) \mathrm{VCt}$ & 901 & & & ${ }_{*} \mathrm{~N}$ & $6 L \mathrm{~N}-\mathrm{Z} \cap \mathrm{MS} \Omega$ & $z L$ & & $s \mathrm{~s} / \mathrm{l}$ & $8 L^{\circ}$ & วฮิะD „.8 LZN & $9 \varepsilon$ \\
\hline & & $* \mathbf{W}$ & II!H ỏnld & s0I & & & $* W$ & $8 L \mathrm{~N}-\mathrm{Z} \Omega \mathrm{MS} \Omega$ & IL & & $\varsigma \mathrm{I} / \mathrm{I}$ & $\varepsilon L^{\circ}$ & LZN"Zn MSn & $9 \varepsilon$ \\
\hline ** & $\mathrm{tl} / \mathrm{l}$ & $08^{\circ} I$ & 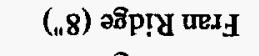 & tol & & & $* W$ & LLN-Zn MSח & $0 L$ & & $t l / l$ & 98 & 9ZN-Zn MSn & $\mathfrak{S \varepsilon}$ \\
\hline \multirow[t]{12}{*}{ ** } & $t 1 / 1$ & $9 L^{\prime} I$ & 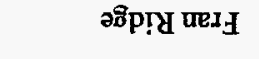 & toI & & & $* \boldsymbol{N}$ & 9LN-Zn MSח & 69 & & $t I /[$ & 06 & SZN-Zn MSO & $t \varepsilon$ \\
\hline & $s I / \mathbb{l}$ & $\$ 8^{\circ}$ & 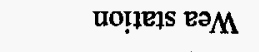 & E0I & & & $* W$ & aฮืeg „. & 89 & & & IN & ged . .t tZN & $\mathfrak{E} \mathfrak{E}$ \\
\hline & SI/I & $6 L^{\circ}$ & UO!̣EIS EәM & E0I & & & $* \boldsymbol{N}$ & $S \angle N-Z n M S \cap$ & 89 & & $t I / I$ & 96 & $\mathrm{tZN}-\mathrm{Zn} M S \cap$ & $\mathfrak{E \varepsilon}$ \\
\hline & $t I / I$ & $0 t^{\circ}$ & uo!̣te]s EəM & 201 & & & $* \mathrm{~W}$ & $\downarrow L \mathrm{~N}-\mathrm{Zn} M \mathrm{MS} \cap$ & $\angle 9$ & & $t I / I$ & sL & LIN-Zn MSn & $z \varepsilon$ \\
\hline & $t I / I$ & $86^{\circ}$ & UO!̣EIS EDM & IOI & & & $* W$ & $\varepsilon L N-Z n M S \cap$ & 99 & & $t I / I$ & $\tau L$ & 9IN-Zn MSn & I $\varepsilon$ \\
\hline & $t l / I$ & 16 & uo!̣EIs ЕәМ & IOI & & & $* \mathbf{W}$ & ZLN-Zn MSO & $\varsigma 9$ & & $t I / I$ & $\angle$ & SIN-Zn MSO & oع \\
\hline & & $* \mathrm{~N}$ & 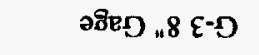 & $00 \mathrm{I}$ & & & $* \mathbf{N}$ & 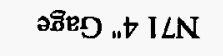 & t9 & & $\downarrow \mathrm{t} / \mathrm{I}$ & $\varepsilon \varsigma$ & ว8ีeפ ..t IIN & $6 z$ \\
\hline & & $* \mathrm{~W}$ & $\varepsilon-D$ & 001 & & & $* W$ & $I \angle N-Z \cap M S \cap$ & t9 & & $t l / l$ & $95^{\circ}$ & IIN-Zn MSO & 62 \\
\hline & SI/I & $65^{\circ}$ & uo!̣EIS вәM & 66 & & $+1 / I$ & 18 & วฮีถญ „ & $\varepsilon 9$ & & & W & วฮีอบ . & $8 z$ \\
\hline & $\mathfrak{s I / I}$ & $9 S^{\circ}$ & toṭełs вәM & 66 & & $t \mathrm{t} / \mathrm{I}$ & t8 & OLN-Zn MSn & $\varepsilon$ & & $t l / l$ & $00 \% 1$ & I6\# NZn 6z-Bn & 82 \\
\hline & SI/I & $9 \mathcal{E}^{\circ}$ & uọ̣edode $\Lambda$ G & 86 & & $+l / I$ & $06^{\circ}$ & 69N-Zn MSn & 29 & $d \forall \Lambda J$ & & $9 \varsigma^{\circ}$ & L6\# NZn ऽZ-Aก & $\angle z$ \\
\hline & & W & uo!̣uมs вəМ & $\angle 6$ & & $\hbar \mathrm{l} / \mathrm{I}$ & $26^{\circ}$ & 89N-Zn MSn & 19 & & $t \mathrm{t} / \mathrm{I}$ & $96^{\circ}$ & 28ิED „t & $9 z$ \\
\hline \multirow[t]{2}{*}{ ** } & $\$ 1 / I$ & s9 & นo!̣ıา's вәM & 96 & & $t I / I$ & $86^{\circ}$ & วฐึอD „ & 09 & & $t \mathrm{t} / \mathrm{l}$ & $06^{\circ}$ & ح6\# NZn sて-Bn & $9 z$ \\
\hline & $t I / I$ & $\mathfrak{E} \boldsymbol{t}^{\cdot}$ & $\varepsilon-H M S \cap$ & §6 & & $t I / I$ & $26^{\circ}$ & L9N-Zn MSח & 09 & & $t l / \mathbf{l}$ & $9 L^{\circ}$ & s8\# NZn sz-Gn & $s z$ \\
\hline ** & $t \mathrm{t} / \mathrm{I}$ & $I 0 \cdot t$ & 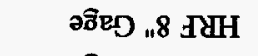 & t6 & & $t \mathrm{t} / \mathrm{I}$ & $\$ 8^{\circ}$ & 99N-Zn MSn & $6 \varsigma$ & & $t l / l$ & $00 \%$ & 09\# NZn sz-3n & $t z$ \\
\hline \multirow[t]{2}{*}{$* *$} & $t I / I$ & $00 \div$ & 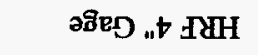 & t6 & dV $\Lambda \exists$ & & $\angle 9^{\circ}$ & $\$ 9 N-Z \cap M S \cap$ & $8 \varsigma$ & & $t \mathrm{t} / \mathrm{I}$ & $86^{\circ}$ & 9S\# NZO sz-G⿵ & $\varepsilon z$ \\
\hline & & & & & ponup & $0 \supset-\mathcal{E} 66$ & $\forall I-Z I X Y Y$ & INVP HO WXOLS & & & & & & \\
\hline $10 \mathrm{~N}$ & $\begin{array}{l}\text { peed } \\
\text { oleo }\end{array}$ & $\begin{array}{l}\text { (seyrul) } \\
\text { junowy }\end{array}$ & $\begin{array}{l}\text { edn\}日|puawou } \\
\text { el|s }\end{array}$ & $\begin{array}{c}\text { opos } \\
\text { uo!l } \\
\text {-E007 }\end{array}$ & selon & $\begin{array}{l}\text { peed } \\
\text { oled }\end{array}$ & $\begin{array}{l}\text { (seysul) } \\
\text { junowy }\end{array}$ & $\begin{array}{l}\text { ednie|puewou } \\
\text { eแIS }\end{array}$ & $\begin{array}{c}\text { epos } \\
\text { Lo|l } \\
\text {-8007 }\end{array}$ & selon & $\begin{array}{l}\text { peed } \\
\text { elea }\end{array}$ & $\begin{array}{l}\text { (soyoul) } \\
\text { junowy }\end{array}$ & $\begin{array}{l}\text { ejnzepuewou } \\
\text { өl/s }\end{array}$ & $\begin{array}{c}\text { opos } \\
\text { UO!! } \\
\text {-e007 }\end{array}$ \\
\hline
\end{tabular}


Table 2. Summary of precipitation data from the nonrecording gage network at Yucca Mountain, Nevada, for water years 1992 and 1993--Continued

\begin{tabular}{|c|c|c|c|c|c|c|c|c|c|c|c|c|c|c|}
\hline $\begin{array}{l}\text { Loca- } \\
\text { tion } \\
\text { code }\end{array}$ & $\begin{array}{c}\text { Site } \\
\text { nomenclature }\end{array}$ & $\begin{array}{l}\text { Amount } \\
\text { (inches) }\end{array}$ & $\begin{array}{l}\text { Date } \\
\text { read }\end{array}$ & Notes & $\begin{array}{l}\text { Loca- } \\
\text { tion } \\
\text { code }\end{array}$ & $\begin{array}{c}\text { Site } \\
\text { nomenclature }\end{array}$ & $\begin{array}{l}\text { Amount } \\
\text { (Inches) }\end{array}$ & $\begin{array}{l}\text { Date } \\
\text { read }\end{array}$ & Notes & $\begin{array}{l}\text { Loca- } \\
\text { tion } \\
\text { code }\end{array}$ & $\begin{array}{c}\text { Site } \\
\text { nomenclature }\end{array}$ & $\begin{array}{l}\text { Amount } \\
\text { (inches) }\end{array}$ & $\begin{array}{l}\text { Date } \\
\text { read }\end{array}$ & Notes \\
\hline \multicolumn{15}{|c|}{ STORM OF JANUARY 17-18, 1993} \\
\hline 1 & UE-25 UZN \#1 & 1.55 & $1 / 20$ & & 38 & USW UZ-N36 & 1.62 & $1 / 20$ & & 73 & N80 4" Gage & 2.01 & $1 / 26$ & ** \\
\hline 2 & UE-25 UZN \#2 & 1.60 & $1 / 20$ & & 39 & USW UZ-N37 & M & & & 74 & USW UZ-N81 & 1.60 & $1 / 26$ & ** \\
\hline 3 & UE-25 UZN \#3 & 1.60 & $1 / 20$ & & 39 & N37 4" Gage & $\mathbf{M}$ & & & 75 & USW UZ-N82 & 1.40 & $1 / 26$ & ** \\
\hline 4 & UE-25 UZN \#4 & 1.69 & $1 / 20$ & & 40 & USW UZ-N40 & 1.50 & $1 / 20$ & & 76 & USW UZ-N83 & 1.70 & $1 / 26$ & ** \\
\hline 4 & N4 4" Gage & 1.66 & $1 / 20$ & EVAP & 40 & N40 4" Gage & NI & & & 77 & USW UZ-N84 & 1.55 & & \\
\hline 5 & UE-25 UZN \#5 & 1.62 & $1 / 20$ & & 41 & USW UZ-N41 & 1.65 & $1 / 20$ & & 78 & USW UZ-N86 & 1.75 & $1 / 26$ & ** \\
\hline 6 & UE-25 UZN \#6 & 1.65 & $1 / 20$ & & 42 & USW UZ-N42 & 1.50 & $1 / 20$ & & 79 & USW UZ-N87 & 1.53 & $1 / 26$ & ** \\
\hline 7 & UE-25 UZN $\# 7$ & 1.66 & $1 / 20$ & & 43 & USW UZ-N43 & 1.55 & $1 / 20$ & & 80 & USW UZ-N88 & 1.66 & $1 / 26$ & $\mathrm{EVAP}^{/ * *}$ \\
\hline 8 & UE-25 UZN $\# 8$ & 1.63 & $1 / 20$ & & 43 & N43 4" Gage & NI & & & 81 & USW UZ-N89 & 1.55 & $1 / 26$ & $* *$ \\
\hline 9 & UE-25 UZN \#9 & 1.69 & $1 / 20$ & & 44 & USW UZ-N44 & 1.45 & $1 / 20$ & & 82 & USW UZ-N90 & 1.55 & $1 / 26$ & ** \\
\hline 10 & UE-25 UZN \#10 & 1.54 & $1 / 20$ & & 45 & USW UZ-N45 & 1.56 & $1 / 20$ & & 82 & N90 4" Gage & 1.60 & $1 / 26$ & ** \\
\hline 10 & N10 4" Gage & 1.49 & $1 / 20$ & EVAP & 46 & USW UZ-N46 & .59 & $1 / 20$ & & 83 & USW UZ-N93 & & & \\
\hline 11 & UE-25 UZN \#12 & 1.67 & $1 / 20$ & & 47 & USW UZ-N47 & $\mathbf{M}^{*}$ & & & 84 & USW UZ-N94 & $\mathbf{M}^{*}$ & $1 / 21$ & \\
\hline 12 & UE-25 UZN \#13 & 1.67 & $1 / 20$ & & 48 & USW UZ-N48 & 1.45 & $1 / 25$ & & 85 & USW UZ-N95 & 1.08 & $1 / 20$ & \\
\hline 13 & UE-25 UZN \#14 & 1.55 & $1 / 20$ & & 49 & USW UZ-N49 & 1.45 & $1 / 25$ & & 86 & USW UZ-N96 & .90 & $1 / 21$ & \\
\hline 14 & UE-25 UZN \#18 & $\mathrm{M}^{*}$ & & & 50 & USW UZ-N50 & 1.40 & $1 / 25$ & & 87 & USW UZ-N98 & $\mathrm{M}^{*}$ & $1 / 21$ & \\
\hline 15 & UE-25 UZN \#19 & 1.69 & $1 / 20$ & & 50 & N50 4" Gage & NI & & & 87 & N98 4" Gage & NI & & \\
\hline 16 & UE-25 UZN \#20 & 1.50 & $1 / 20$ & & 51 & USW UZ-N51 & 1.45 & $1 / 20$ & & 88 & USW UZ-13 & .89 & $1 / 20$ & \\
\hline 17 & UE-25 UZN \#21 & 1.50 & $1 / 20$ & & 52 & USW UZ-N52 & 1.40 & $1 / 20$ & & 88 & UZ-13 4" Gage & .88 & $1 / 20$ & \\
\hline 17 & N21 4" Gage & NI & & & 53 & USW UZ-N53 & 1.45 & $1 / 20$ & & 89 & USW GA-1 & $\mathbf{M}^{*}$ & & \\
\hline 18 & UE-25 UZN \#22 & 1.58 & $1 / 20$ & & 54 & USW UZ-N54 & $\mathbf{M}$ & & & 90 & USW G-2 & $\mathbf{M}^{*}$ & & \\
\hline 19 & UE-25 UZN \#23 & 1.52 & $1 / 20$ & & 54 & N54 4" Gage & $\mathbf{M}$ & & & 91 & UE25 WT-4 & 1.53 & $1 / 20$ & \\
\hline 20 & UE-25 UZN \#28 & 1.42 & $1 / 20$ & & 55 & USW UZ-NSS & $\mathbf{M}$ & & & 92 & UE25 WT-18 & 1.44 & $1 / 20$ & EVAP \\
\hline 21 & UE-25 UZN \#29 & 1.42 & $1 / 20$ & & 56 & USW UZ-NS7 & 1.25 & $1 / 20$ & & 93 & USW H-5 & 1.13 & $1 / 20$ & \\
\hline 22 & UE-25 UZN \#30 & 1.50 & $1 / 20$ & & 57 & USW UZ-N64 & 1.24 & $1 / 20$ & & 94 & HRF Wedge & 1.29 & $1 / 20$ & \\
\hline
\end{tabular}




\begin{tabular}{|c|c|c|c|c|c|c|c|c|c|c|c|c|c|c|}
\hline $\begin{array}{l}\text { Loca- } \\
\text { tion } \\
\text { code }\end{array}$ & $\begin{array}{c}\text { Site } \\
\text { nomenclature }\end{array}$ & $\begin{array}{l}\text { Amount } \\
\text { (inches) }\end{array}$ & $\begin{array}{l}\text { Date } \\
\text { read }\end{array}$ & Notes & $\begin{array}{l}\text { Loca- } \\
\text { tion } \\
\text { code }\end{array}$ & $\begin{array}{c}\text { Site } \\
\text { nomenclature }\end{array}$ & $\begin{array}{l}\text { Amount } \\
\text { (Inches) }\end{array}$ & $\begin{array}{l}\text { Date } \\
\text { read }\end{array}$ & Notes & $\begin{array}{l}\text { Loca- } \\
\text { tion } \\
\text { code }\end{array}$ & $\begin{array}{c}\text { Site } \\
\text { nomenclature }\end{array}$ & $\begin{array}{l}\text { Amount } \\
\text { (inches) }\end{array}$ & $\begin{array}{l}\text { Date } \\
\text { read }\end{array}$ & Notes \\
\hline \multicolumn{15}{|c|}{ STORM OF JANUARY 17-18, 1993-Continued } \\
\hline 23 & UE-25 UZN \#56 & 1.40 & $1 / 20$ & & 58 & USW UZ-N65 & 1.16 & $1 / 20$ & & 94 & HRF 4" Gage & 1.44 & $1 / 20$ & \\
\hline 24 & UE-25 UZN \#60 & 1.45 & $1 / 20$ & & 59 & USW UZ-NGE & 1.31 & $1 / 20$ & & 94 & HRr g" Gage & 1.45 & $1 / 20$ & \\
\hline 25 & UE-25 UZN \#85 & 1.29 & $1 / 20$ & & 60 & USW UZ-N67 & 1.30 & $1 / 20$ & & 95 & USW H-3 & .95 & $1 / 20$ & \\
\hline 26 & UE-25 UZN \#92 & 1.55 & $1 / 20$ & & 60 & N67 4" Gage & 1.34 & $1 / 20$ & & 96 & Wea station & 1.00 & $1 / 20$ & \\
\hline 26 & N92 4" Gage & $\mathbf{M}$ & & & 61 & USW UZ-N68 & 1.29 & $1 / 20$ & & 97 & Wea station & $\mathrm{M}$ & $1 / 20$ & \\
\hline 27 & UE-25 UZN \#97 & 1.52 & $1 / 20$ & & 62 & USW UZ-N69 & 1.28 & $1 / 20$ & & 98 & Evaporation & 1.18 & $1 / 20$ & \\
\hline 28 & UE-29 UZN \#91 & 2.05 & $1 / 20$ & & 63 & USW UZ-N70 & $\mathbf{M}^{*}$ & & & 99 & Wea station & 1.04 & $1 / 20$ & \\
\hline 28 & N91 4" Gage & $\mathbf{M}$ & & & 63 & N70 4" Gage & $\mathrm{M}^{*}$ & & & 99 & Wea station & 1.02 & $1 / 20$ & \\
\hline 29 & USW UZ-N11 & 1.40 & $1 / 20$ & & 64 & USW UZ-N71 & 1.10 & $1 / 20$ & $* *$ & 100 & G-3 & 1.84 & $1 / 20$ & \\
\hline 29 & N11 4" Gage & 1.37 & $1 / 20$ & & 64 & N71 4" Gage & 1.05 & $1 / 20$ & $* *$ & 100 & G-3 8" Gage & 1.91 & $1 / 20$ & \\
\hline 30 & USW UZ-N15 & 1.57 & $1 / 20$ & & 65 & USW UZ-N72 & 1.00 & $1 / 27$ & $* *$ & 101 & Wea station & 1.32 & $1 / 20$ & \\
\hline 31 & USW UZ-N16 & 1.48 & $1 / 20$ & & 66 & USW UZ-N73 & 1.10 & $1 / 27$ & $* *$ & 101 & Wea station & 1.37 & $1 / 20$ & \\
\hline 32 & USW UZ-N17 & 1.54 & $1 / 20$ & & 67 & USW UZ-N74 & 1.03 & $1 / 27$ & $* *$ & 102 & Wea station & 1.23 & $1 / 20$ & \\
\hline 33 & USW UZ-N24 & $\mathrm{M}^{*}$ & & & 68 & USW UZ-N75 & 1.15 & $1 / 27$ & $* *$ & 103 & Wea station & 1.30 & $1 / 20$ & \\
\hline 33 & N24 4" Gage & NI & & & 68 & N75 4" Gage & .55 & $1 / 27$ & $\mathrm{EVAP} / * *$ & 103 & Wea station & 1.42 & $1 / 20$ & \\
\hline 34 & USW UZ-N25 & $\mathbf{M}^{*}$ & & & 69 & USW UZ-N76 & 1.15 & $1 / 27$ & $* *$ & 104 & Fran Ridge & 1.23 & $1 / 20$ & \\
\hline 35 & USW UZ-N26 & $\mathbf{M}^{*}$ & & & 70 & USW UZ-N77 & 1.35 & $1 / 26$ & $* *$ & 104 & Fran Ridge (8") & 1.26 & $1 / 20$ & \\
\hline 36 & USW UZ-N27 & 1.31 & $1 / 20$ & & 71 & USW UZ-N78 & 1.65 & $1 / 26$ & $* *$ & 105 & Plug Hill & 1.00 & $1 / 20$ & \\
\hline 36 & N27 8" Gage & 1.25 & $1 / 20$ & & 72 & USW UZ-N79 & 1.63 & $1 / 26$ & $* *$ & 106 & 4JA (8") & $\mathbf{M}^{*}$ & & \\
\hline \multirow[t]{2}{*}{37} & USW UZ-N35 & 1.56 & $1 / 20$ & & 73 & USW UZ-N80 & 1.87 & $1 / 26$ & $* *$ & 107 & Knothead Gap & 1.30 & $1 / 20$ & \\
\hline & & & & & & & & & & 108 & Upper Forty Mile & 1.65 & $1 / 20$ & EVAP \\
\hline
\end{tabular}


Table 2. Summary of precipitation data from the nonrecording gage network at Yucca Mountain, Nevada, for water years 1992 and $1993-$ Continued

\begin{tabular}{|c|c|c|c|c|c|c|c|c|c|c|c|c|c|c|}
\hline $\begin{array}{l}\text { Loca- } \\
\text { tion } \\
\text { code }\end{array}$ & $\begin{array}{c}\text { Site } \\
\text { nomenclature }\end{array}$ & $\begin{array}{l}\text { Amount } \\
\text { (inches) }\end{array}$ & $\begin{array}{l}\text { Date } \\
\text { read }\end{array}$ & Notes & $\begin{array}{l}\text { Loca- } \\
\text { tion } \\
\text { code }\end{array}$ & $\begin{array}{c}\text { Site } \\
\text { nomenclature }\end{array}$ & $\begin{array}{l}\text { Amount } \\
\text { (inches) }\end{array}$ & $\begin{array}{l}\text { Date } \\
\text { read }\end{array}$ & Notes & $\begin{array}{l}\text { Loca- } \\
\text { tjon } \\
\text { code }\end{array}$ & $\begin{array}{c}\text { Site } \\
\text { nomenclature }\end{array}$ & $\begin{array}{l}\text { Amount } \\
\text { (Inches) }\end{array}$ & $\begin{array}{l}\text { Date } \\
\text { read }\end{array}$ & Notes \\
\hline \multicolumn{15}{|c|}{ STORM OF JANUARY 30-31, 1993} \\
\hline 1 & UE-25 UZN \#1 & .23 & $2 / 2$ & & 38 & USW UZ-N36 & $\mathrm{M}^{*}$ & & & 73 & N80 4" Gage & .15 & $2 / 4$ & \\
\hline 2 & UE-25 UZN \#2 & .12 & $2 / 2$ & & 39 & USW UZ-N37 & $\mathbf{M}^{\prime}$ & & & 74 & USW UZ-N81 & .07 & $2 / 4$ & \\
\hline 3 & UE-25 UZN \#3 & .15 & $2 / 2$ & & 39 & N37 4" Gage & $\mathbf{M}^{*}$ & & & 75 & USW UZ-N82 & .07 & $2 / 4$ & \\
\hline 4 & UE-25 UZN \#4 & .19 & $2 / 2$ & & 40 & USW UZ-N40 & $\mathbf{M}^{*}$ & & & 76 & USW UZ-N83 & $\mathbf{M}^{*}$ & & \\
\hline 4 & N4 4" Gage & .10 & $2 / 2$ & EVAP & 40 & N40 4" Gage & .15 & $2 / 2$ & & 77 & USW UZ-N84 & & & \\
\hline 5 & UE-25 UZN \#5 & .18 & $2 / 2$ & & 41 & USW UZ-N4I & NI & & & 78 & USW UZ-N86 & $\mathbf{M}^{*}$ & & \\
\hline 6 & UE-25 UZN \#6 & .20 & $2 / 2$ & & 42 & USW UZ-N42 & .15 & $2 / 2$ & & 79 & USW UZ-N87 & .09 & $2 / 4$ & \\
\hline 7 & UE-25 UZN \#7 & .20 & $2 / 2$ & & 43 & USW UZ-N43 & .20 & $2 / 2$ & & 80 & USW UZ-N88 & .12 & $2 / 4$ & \\
\hline 8 & UE-25 UZN \#8 & .20 & $2 / 2$ & & 43 & N43 4" Gage & .25 & $2 / 2$ & & 81 & USW UZ-N89 & .10 & $2 / 4$ & \\
\hline 9 & UE-25 UZN $\# 9$ & .20 & $2 / 2$ & & 44 & USW UZ-N44 & NI & & & 82 & USW UZ-N90 & .10 & $2 / 4$ & \\
\hline 10 & UE-25 UZN \#10 & .21 & $2 / 2$ & & 45 & USW UZ-N45 & .20 & $2 / 2$ & & 82 & N90 4" Gage & .12 & $2 / 4$ & \\
\hline 10 & N10 4" Gage & .15 & $2 / 2$ & EVAP & 46 & USW UZ-N46 & .25 & $2 / 2$ & & 83 & USW UZ-N93 & & & \\
\hline 11 & UE-25 UZN \#12 & .10 & $2 / 2$ & & 47 & USW UZ-N47 & .08 & $2 / 2$ & EVAP & 84 & USW UZ-N94 & $\mathbf{M}^{*}$ & & \\
\hline 12 & UE-25 UZN \#13 & .24 & $2 / 2$ & & 48 & USW UZ-N48 & 1.40 & $2 / 2$ & ** & 85 & USW UZ-N95 & $\mathbf{M}^{*}$ & & \\
\hline 13 & UE-25 UZN \#14 & .20 & $2 / 2$ & & 49 & USW UZ-N49 & .15 & $2 / 2$ & & 86 & USW UZ-N96 & $\mathbf{M}^{*}$ & & \\
\hline 14 & UE-25 UZN \#18 & $\mathbf{M}^{*}$ & & & 50 & USW UZ-N50 & .05 & $2 / 2$ & & 87 & USW UZ-N98 & $\mathbf{M}^{*}$ & & \\
\hline 15 & UE-25 UZN \#19 & .13 & $2 / 2$ & & 50 & N50 4" Gage & .10 & $2 / 2$ & & 87 & N98 4" Gage & NI & & \\
\hline 16 & UE-25 UZN \#20 & .20 & $2 / 2$ & & 51 & USW UZ-N51 & NI & & & 88 & USW UZ-13 & $\mathbf{M}^{*}$ & & \\
\hline 17 & UE-25 UZN \#21 & .15 & $2 / 2$ & & 52 & USW UZ-N52 & .10 & $2 / 2$ & & 88 & UZ-13 4" Gage & $\mathbf{M}^{*}$ & & \\
\hline 17 & N21 4" Gage & NI & & & 53 & USW UZ-N53 & .15 & $2 / 2$ & & 89 & USW GA-1 & $\mathbf{M}^{*}$ & & \\
\hline 18 & UE-25 UZN \#22 & .19 & $2 / 2$ & & 54 & USW UZ-N54 & .10 & $2 / 2$ & & 90 & USW G-2 & $\mathbf{M}^{*}$ & & \\
\hline 19 & UE-25 UZN \#23 & .10 & $2 / 2$ & & 54 & N54 4" Gage & .08 & $2 / 2$ & & 91 & UE25 WT-4 & $\mathbf{M}^{*}$ & & \\
\hline 20 & UE-25 UZN \#28 & .13 & & & 55 & USW UZ-N55 & .09 & $2 / 2$ & & 92 & UE25 WT-18 & $\mathbf{M}^{*}$ & & \\
\hline 21 & UE-25 UZN $\# 29$ & .20 & $2 / 2$ & & 56 & USW UZ-N57 & $\mathbf{M}^{*}$ & & & 93 & USW H-5 & $\mathbf{M}^{*}$ & & \\
\hline 22 & UE-25 UZN \#30 & .20 & $2 / 2$ & & 57 & USW UZ-N64 & $\mathbf{M}^{*}$ & & & 94 & HRF Wedge & $\mathbf{M}^{*}$ & & \\
\hline
\end{tabular}




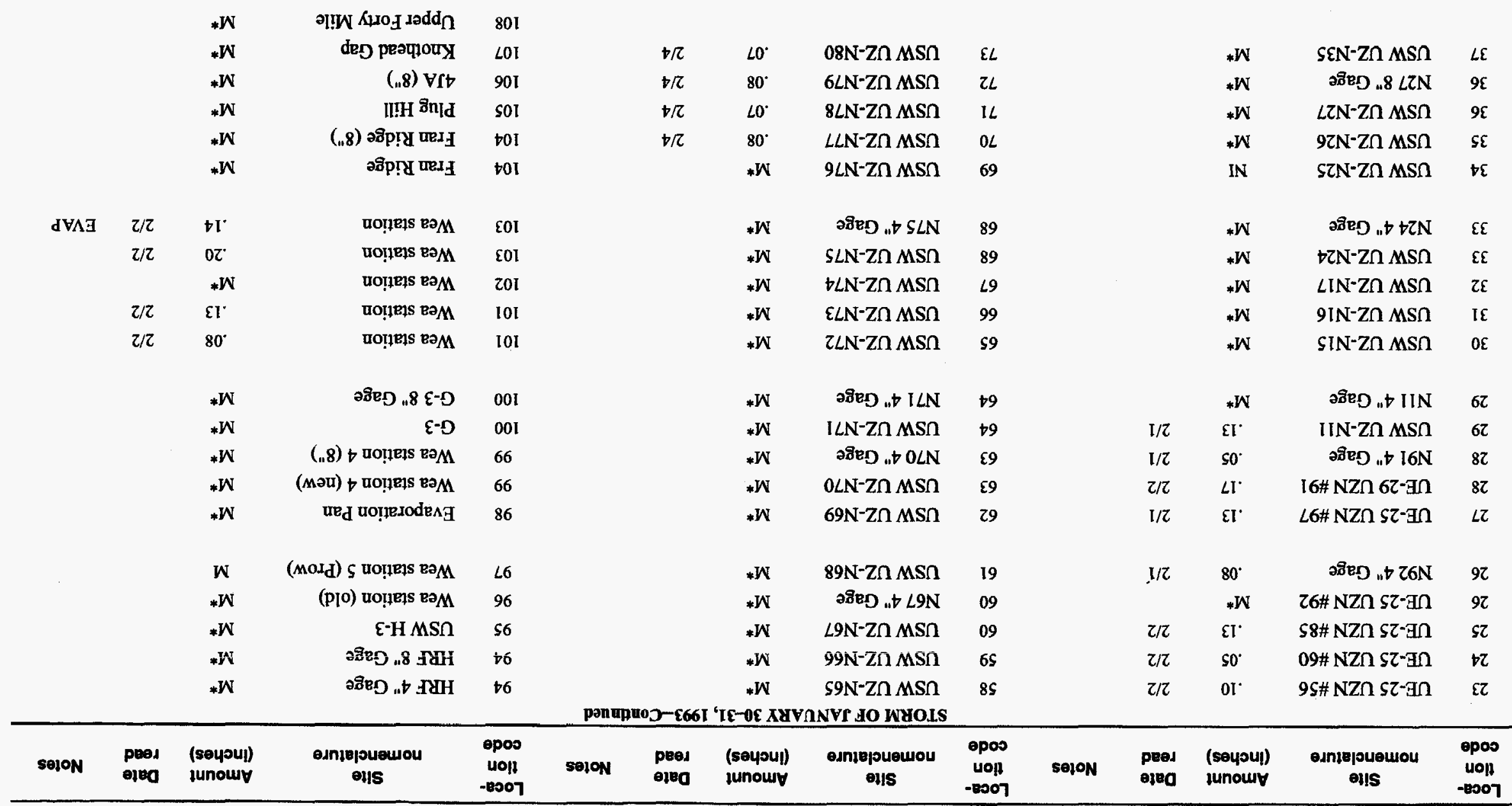


Table 2. Summary of precipitation data from the nonrecording gage network at Yucca Mountain, Nevada, for water years 1992 and 1993 --Continued

\begin{tabular}{|c|c|c|c|c|c|c|c|c|c|c|c|c|c|c|}
\hline $\begin{array}{l}\text { Loca- } \\
\text { tion } \\
\text { code }\end{array}$ & $\begin{array}{c}\text { SIte } \\
\text { nomenclature }\end{array}$ & $\begin{array}{l}\text { Amount } \\
\text { (inches) }\end{array}$ & $\begin{array}{l}\text { Date } \\
\text { read }\end{array}$ & Notes & $\begin{array}{l}\text { Loca- } \\
\text { tion } \\
\text { code }\end{array}$ & $\begin{array}{c}\text { Site } \\
\text { nomenclature }\end{array}$ & $\begin{array}{l}\text { Amount } \\
\text { (inches) }\end{array}$ & $\begin{array}{l}\text { Date } \\
\text { read }\end{array}$ & Notes & $\begin{array}{l}\text { Loca- } \\
\text { tion } \\
\text { code }\end{array}$ & $\begin{array}{c}\text { Site } \\
\text { nomenclature }\end{array}$ & $\begin{array}{l}\text { Amount } \\
\text { (inches) }\end{array}$ & $\begin{array}{l}\text { Date } \\
\text { read }\end{array}$ & Notes \\
\hline \multicolumn{15}{|c|}{ STORM OF FEBRUARY 7-8, 1993} \\
\hline 1 & UE-25 UZN \#1 & 1.75 & $2 / 11$ & EVAP & 38 & USW UZ-N36 & 1.41 & $2 / 11$ & ** & 73 & N80 4" Gage & 1.36 & & \\
\hline 2 & UE-25 UZN \#2 & 1.52 & $2 / 11$ & EVAP & 39 & USW UZ-N37 & 3.35 & $2 / 12$ & ** & 74 & USW UZ-N81 & 1.28 & & \\
\hline 3 & UE-25 UZN \#3 & 1.58 & $2 / 12$ & & 39 & N37 4" Gage & 1.72 & $2 / 12$ & $* *$ & 75 & USW UZ-N82 & 1.28 & $2 / 12$ & \\
\hline 4 & UE-25 UZN \#4 & 1.67 & $2 / 11$ & EVAP & 40 & USW UZ-N40 & 1.45 & $2 / 12$ & & 76 & USW UZ-N83 & 1.44 & $2 / 12$ & \\
\hline 4 & N4 4" Gage & 1.37 & $2 / 11$ & EVAP & 40 & N40 4" Gage & $\mathrm{NI}$ & & & 77 & USW UZ-N84 & 1.29 & $2 / 12$ & \\
\hline 5 & UE-25 UZN \#5 & 1.50 & $2 / 12$ & & 41 & USW UZ-N41 & 1.55 & $2 / 12$ & & 78 & USW UZ-N86 & 1.48 & $2 / 12$ & $* *$ \\
\hline 6 & UE-25 UZN \#6 & 1.52 & $2 / 12$ & & 42 & USW UZ-N42 & 1.55 & $2 / 12$ & & 79 & USW UZ-N87 & 1.27 & $2 / 12$ & \\
\hline 7 & UE-25 UZN \#7 & 1.70 & $2 / 11$ & & 43 & USW UZ-N43 & 1.53 & $2 / 16$ & & 80 & USW UZ-N88 & 1.39 & $2 / 12$ & EVAP \\
\hline 8 & UE-25 UZN \#8 & 1.52 & $2 / 12$ & & 43 & N43 4" Gage & NI & & & 81 & USW UZ-N89 & 1.30 & $2 / 12$ & \\
\hline 9 & UE-25 UZN \#9 & 1.69 & $2 / 11$ & EVAP & 44 & USW UZ-N44 & 1.60 & $2 / 16$ & & 82 & USW UZ-N90 & 1.31 & $2 / 12$ & \\
\hline 10 & UE-25 UZN \#10 & 1.744 & $2 / 11$ & EVAP & 45 & USW UZ-N45 & 1.45 & $2 / 16$ & & 82 & N90 4" Gage & 1.38 & $2 / 12$ & \\
\hline 10 & N10 4" Gage & 1.46 & $2 / 11$ & EVAP & 46 & USW UZ-N46 & 1.32 & $2 / 16$ & & 83 & USW UZ-N93 & & & \\
\hline 11 & UE-25 UZN \#12 & 1.59 & $2 / 12$ & & 47 & USW UZ-N47 & 1.54 & $2 / 16$ & EVAP & 84 & USW UZ-N94 & 1.53 & $2 / 11$ & \\
\hline 12 & UE-25 UZN \#13 & 1.49 & $2 / 12$ & EVAP & 48 & USW UZ-N48 & 1.54 & $2 / 16$ & & 85 & USW UZ-N95 & 1.51 & $2 / 11$ & \\
\hline 13 & UE-25 UZN \#14 & 1.40 & $2 / 12$ & & 49 & USW UZ-N49 & 1.50 & $2 / 16$ & & 86 & USW UZ-N96 & 1.54 & $2 / 11$ & ** \\
\hline 14 & UE-25 UZN \#18 & 3.22 & $2 / 16$ & $* *$ & 50 & USW UZ-N50 & 1.46 & $2 / 16$ & & 87 & USW UZ-N98 & 3.30 & $2 / 12$ & \\
\hline 15 & UE-25 UZN \#19 & 1.51 & $2 / 10$ & & 50 & N50 4" Gage & $\mathrm{NI}$ & & & 87 & N98 4" Gage & $\mathrm{NI}$ & & \\
\hline 16 & UE-25 UZN \#20 & 1.52 & $2 / 16$ & & 51 & USW UZ-N51 & 1.48 & $2 / 16$ & & 88 & USW UZ-13 & 1.30 & $2 / 11$ & ** \\
\hline 17 & UE-25 UZN \#21 & 1.58 & $2 / 16$ & & 52 & USW UZ-N52 & 1.41 & $2 / 16$ & & 88 & UZ-134" Gage & 1.14 & $2 / 11$ & $\operatorname{EVAP} / * *$ \\
\hline 17 & N21 4" Gage & NI & & & 53 & USW UZ-N53 & 1.48 & $2 / 16$ & & 89 & USW GA-1 & 1.97 & $2 / 11$ & EVAP/** \\
\hline 18 & UE-25 UZN \#22 & 1.57 & $2 / 10$ & & 54 & USW UZ-N54 & 1.53 & $2 / 16$ & & 90 & USW G-2 & 2.07 & $2 / 11$ & ** \\
\hline 19 & UE-25 UZN \#23 & 1.55 & $2 / 16$ & & 54 & N54 4" Gage & 1.52 & $2 / 16$ & & 91 & UE25 WT-4 & 1.50 & $2 / 11$ & ** \\
\hline 20 & UE-25 UZN \#28 & 1.60 & $2 / 16$ & & 55 & USW UZ-N55 & 1.51 & $2 / 10$ & & 92 & UE25 WT-18 & 1.15 & $2 / 11$ & $\mathrm{EVAP}^{/ * *}$ \\
\hline 21 & UE-25 UZN \#29 & 1.55 & $2 / 16$ & & 56 & USW UZ-N57 & 1.51 & $2 / 10$ & @ & 93 & USW H-5 & $\mathrm{M}^{*}$ & & \\
\hline 22 & UE-25 UZN \#30 & 1.60 & $2 / 16$ & & 57 & USW UZ-N64 & 1.35 & $2 / 11$ & $\mathrm{EVAP}^{/ * *}$ & 94 & HRF Wedge & 1.63 & $2 / 10$ & $* *$ \\
\hline
\end{tabular}


8 Table 2. Summary of precipitation data from the nonrecording gage network at Yucca Mountain, Nevada, for water years 1992 and $1993--$ Continued

\begin{tabular}{|c|c|c|c|c|c|c|c|c|c|c|c|c|c|c|}
\hline $\begin{array}{l}\text { Loca- } \\
\text { tlon } \\
\text { code }\end{array}$ & $\begin{array}{c}\text { Site } \\
\text { nomenclature }\end{array}$ & $\begin{array}{l}\text { Amount } \\
\text { (inches) }\end{array}$ & $\begin{array}{l}\text { Date } \\
\text { read }\end{array}$ & Notes & $\begin{array}{l}\text { Lock- } \\
\text { tion } \\
\text { code }\end{array}$ & $\begin{array}{c}\text { Site } \\
\text { nomenclature }\end{array}$ & $\begin{array}{l}\text { Amount } \\
\text { (inches) }\end{array}$ & $\begin{array}{l}\text { Date } \\
\text { read }\end{array}$ & Notes & $\begin{array}{l}\text { Loca- } \\
\text { tion } \\
\text { code }\end{array}$ & $\begin{array}{c}\text { Site } \\
\text { nomenclature }\end{array}$ & $\begin{array}{l}\text { Amount } \\
\text { (Inches) }\end{array}$ & $\begin{array}{l}\text { Date } \\
\text { read }\end{array}$ & Notes \\
\hline \multicolumn{15}{|c|}{ STORM OF FEBRUARY 7-8, 1993-Continued } \\
\hline 23 & UE-25 UZN \#56 & 1.55 & $2 / 16$ & & 58 & USW UZ-N65 & 1.47 & $2 / 11$ & ** & 94 & HRF 4" Gage & 1.74 & $2 / 10$ & \\
\hline 24 & UE-25 UZN \#60 & 1.52 & $2 / 10$ & & 59 & USW UZ-N̄66 & 1.40 & $2 / 11$ & $* *$ & 94 & HRF 8" Gage & 1.62 & $2 / 10$ & \\
\hline 25 & UE-25 UZN \#85 & 1.13 & $2 / 11$ & ** & 60 & USW UZ-N67 & 1.45 & $2 / 11$ & $* *$ & 95 & USW H-3 & 1.05 & & \\
\hline 26 & UE-25 UZN \#92 & 1.17 & $2 / 12$ & & 60 & N67 4" Gage & 1.48 & $2 / 11$ & ** & 96 & Wea station & 1.30 & $2 / 11$ & \\
\hline 26 & N92 4" Gage & 1.31 & $2 / 12$ & & 61 & USW UZ-N68 & 1.49 & $2 / 11$ & $* *$ & 97 & Wea station 5 (Prow) & & & \\
\hline 27 & UE-25 UZN \#97 & 1.61 & $2 / 10$ & & 62 & USW UZ-N69 & 1.46 & $2 / 11$ & $* *$ & 98 & Evaporation & 1.37 & $2 / 11$ & \\
\hline 28 & UE-29 UZN \#91 & 1.40 & $2 / 12$ & & 63 & USW UZ-N70 & 2.95 & $2 / 12$ & ** & 99 & Wea station & 1.40 & $2 / 11$ & \\
\hline 28 & N91 4" Gage & 1.42 & $2 / 12$ & & 63 & N70 4" Gage & 1.79 & $2 / 11$ & $\operatorname{EVAP}^{* * *}$ & 99 & Wea station & 1.30 & $2 / 11$ & \\
\hline 29 & USW UZ-N11 & 1.55 & $2 / 11$ & $* *$ & 64 & USW UZ-N71 & 1.35 & $2 / 11$ & ** & 100 & G-3 & 1.36 & & \\
\hline 29 & N11 4" Gage & 1.42 & $2 / 11$ & $\operatorname{EVAP}^{/ * *}$ & 64 & N71 4" Gage & 1.18 & $2 / 11$ & $\operatorname{EVAP}^{* * *}$ & 100 & G-3 8" Gage & 1.30 & $2 / 11$ & EVAP/** \\
\hline 30 & USW UZ-N15 & 1.48 & $2 / 11$ & $\mathrm{EVAP} / * *$ & 65 & USW UZ-N72 & 1.29 & $2 / 11$ & $* *$ & 101 & Wea station & 1.65 & & \\
\hline 31 & USW UZ-N16 & 1.47 & $2 / 11$ & $\mathrm{EVAP}^{/ * *}$ & 66 & USW UZ-N73 & 1.36 & $2 / 11$ & $* *$ & 101 & Wea station & 1.42 & & \\
\hline 32 & USW UZ-N17 & 1.46 & $2 / 11$ & $\mathrm{EVAP}^{/ * *}$ & 67 & USW UZ-N74 & 1.32 & $2 / 11$ & $* *$ & 102 & Wea station & 1.58 & & \\
\hline 33 & USW UZ-N24 & 3.28 & $2 / 12$ & ** & 68 & USW UZ-N75 & 1.39 & $2 / 11$ & $* *$ & 103 & Wea station & 1.40 & & \\
\hline 33 & N24 4" Gage & $\mathrm{NI}$ & & & 68 & N75 4" Gage & 1.20 & $2 / 11$ & $\mathrm{EVAP}^{\prime * *}$ & 103 & Wea station $3\left(8^{*}\right)$ & 1.44 & $2 / 10$ & EVAP \\
\hline 34 & USW UZ-N25 & 3.15 & $2 / 12$ & $* *$ & 69 & USW UZ-N76 & 1.41 & $2 / 12$ & $* *$ & 104 & Fran Ridge & 1.08 & & \\
\hline 35 & USW UZ-N26 & 3.14 & $2 / 12$ & $* *$ & 70 & USW UZ-N77 & 1.22 & $2 / 12$ & & 104 & Fran Ridge (8") & 1.22 & $2 / 12$ & \\
\hline 36 & USW UZ-N27 & 1.48 & $2 / 11$ & ** & 71 & USW UZ-N78 & 1.28 & $2 / 12$ & & 105 & Plug Hill & 1.18 & $2 / 12$ & \\
\hline 36 & N27 8" Gage & 1.35 & $2 / 11$ & EVAP/** & 72 & USW UZ-N79 & 1.32 & $2 / 12$ & & 106 & 4JA (8") & 1.63 & & \\
\hline \multirow[t]{2}{*}{37} & USW UZ-N35 & 1.68 & $2 / 10$ & ** & 73 & USW UZ-N80 & 1.43 & $2 / 12$ & & 107 & Knothead Gap & 1.51 & $2 / 12$ & \\
\hline & & & & & & & & & & 108 & Upper Forty Mile & .56 & $2 / 10$ & EVAP \\
\hline
\end{tabular}


Table 2. Summary of precipitation data from the nonrecording gage network at Yucca Mountain, Nevada, for water years 1992 and 1993--Continued

\begin{tabular}{|c|c|c|c|c|c|c|c|c|c|c|c|c|c|c|}
\hline $\begin{array}{l}\text { Loca- } \\
\text { tion } \\
\text { code }\end{array}$ & $\begin{array}{c}\text { Site } \\
\text { nomenclature }\end{array}$ & $\begin{array}{l}\text { Amount } \\
\text { (inches) }\end{array}$ & $\begin{array}{l}\text { Date } \\
\text { read }\end{array}$ & Notes & $\begin{array}{l}\text { Loca- } \\
\text { tion } \\
\text { code }\end{array}$ & $\begin{array}{c}\text { Site } \\
\text { nomenelature }\end{array}$ & $\begin{array}{l}\text { Amount } \\
\text { (inches) }\end{array}$ & $\begin{array}{l}\text { Date } \\
\text { read }\end{array}$ & Notes & $\begin{array}{l}\text { Loca- } \\
\text { tion } \\
\text { code }\end{array}$ & $\begin{array}{c}\text { Site } \\
\text { nomenclature }\end{array}$ & $\begin{array}{l}\text { Amount } \\
\text { (inches) }\end{array}$ & $\begin{array}{l}\text { Date } \\
\text { read }\end{array}$ & Notes \\
\hline \multicolumn{15}{|c|}{ STORM OF FEBRUARY 18-19, 1993} \\
\hline 1 & UE-25 UZN \#1 & .58 & $2 / 22$ & & 38 & USW UZ-N36 & $\mathrm{M}^{*}$ & & & 73 & N80 4" Gage & $\mathrm{M}^{*}$ & & \\
\hline 2 & UE-25 UZN \#2 & .36 & $2 / 22$ & & 39 & USW UZ-N37 & $\mathrm{M}^{*}$ & & & 74 & USW UZ-N81 & $\mathbf{M}^{*}$ & & \\
\hline 3 & UE-25 UZN \#3 & .52 & $2 / 22$ & & 39 & N37 4" Gage & $\mathrm{M}^{*}$ & & & 75 & USW UZ-N82 & $\mathbf{M}^{*}$ & & \\
\hline 4 & UE-25 UZN \#4 & .60 & $2 / 22$ & & 40 & USW UZ-N40 & $\mathrm{M}^{*}$ & & & 76 & USW UZ-N83 & $\mathbf{M}^{*}$ & & \\
\hline 4 & N4 4" Gage & .41 & $2 / 22$ & EVAP & 40 & N40 4" Gage & NI & & & 77 & USW UZ-N84 & $\mathbf{M}^{*}$ & & \\
\hline 5 & UE-25 UZN \#5 & .52 & 2/22 & & 41 & USW UZ-N41 & $\mathrm{M}^{*}$ & & & 78 & USW UZ-N86 & $\mathrm{M}^{*}$ & & \\
\hline 6 & UE-25 UZN \#6 & .54 & $2 / 22$ & & 42 & USW UZ-N42 & $\mathrm{M}^{*}$ & & & 79 & USW UZ-N87 & $\mathrm{M}^{*}$ & & \\
\hline 7 & UE-25 UZN \#7 & .59 & $2 / 22$ & & 43 & USW UZ-N43 & $\mathrm{M}^{*}$ & & & 80 & USW UZ-N88 & $\mathbf{M}^{*}$ & & \\
\hline 8 & UE-25 UZN \#8 & .54 & $2 / 22$ & & 43 & N43 4" Gage & NI & & & 81 & USW UZ-N89 & $\mathbf{M}^{*}$ & & \\
\hline 9 & UE-25 UZN \#9 & .59 & $2 / 22$ & & 44 & USW UZ-N44 & $\mathbf{M}^{*}$ & & & 82 & USW UZ-N90 & $\mathbf{M}^{*}$ & & \\
\hline 10 & UE-25 UZN \#10 & .55 & $2 / 22$ & & 45 & USW UZ-N45 & $\mathrm{M}^{*}$ & @ & & 82 & N90 4" Gage & $\mathrm{M}^{*}$ & & \\
\hline 10 & N10 4" Gage & .45 & $2 / 22$ & EVAP & 46 & USW UZ-N46 & .56 & $2 / 22$ & & 83 & USW UZ-N93 & & & \\
\hline 11 & UE-25 UZN \#12 & .54 & $2 / 22$ & & 47 & USW UZ-N47 & .52 & $2 / 22$ & & 84 & USW UZ-N94 & $\mathbf{M}^{*}$ & & \\
\hline 12 & UE-25 UZN \#13 & .60 & $2 / 22$ & & 48 & USW UZ-N48 & $\mathbf{M}^{*}$ & & & 85 & USW UZ-N9S & $\mathbf{M}^{*}$ & & \\
\hline 13 & UE-25 UZN \#14 & .50 & $2 / 22$ & & 49 & USW UZ-N49 & $\mathrm{M}^{*}$ & & & 86 & USW UZ-N96 & $\mathrm{M}^{*}$ & & \\
\hline 14 & UE-25 UZN \#18 & $\mathbf{M}^{*}$ & & & 50 & USW UZ-N50 & $\mathrm{M}^{*}$ & & & 87 & USW UZ-N98 & $\mathbf{M}^{*}$ & & \\
\hline 15 & UE-25 UZN\#19 & $\mathbf{M}^{*}$ & & & 50 & N50 4" Gage & NI & & & 87 & N98 4" Gage & $\dot{\mathrm{N} I}$ & & \\
\hline 16 & UE-25 UZN \#20 & $\mathbf{M}^{*}$ & & & 51 & USW UZ-N5 I & $\mathbf{M}^{*}$ & @ & & 88 & USW UZ-13 & $\mathbf{M}^{*}$ & & \\
\hline 17 & UE-25 UZN \#21 & $\mathrm{M}^{*}$ & & & 52 & USW UZ-N52 & $\mathbf{M}^{*}$ & & & 88 & UZ-13 4" Gage & $\mathbf{M}^{*}$ & & \\
\hline 17 & N21 4" Gage & NI & & & 53 & USW UZ-N53 & $\mathrm{M}^{*}$ & & & 89 & USW GA-1 & $\mathbf{M}^{*}$ & & \\
\hline 18 & UE-25 UZN \#22 & $\mathrm{M}^{*}$ & & & 54 & USW UZ-N54 & $\mathbf{M}^{*}$ & & & 90 & USW G-2 & $\mathrm{M}^{*}$ & & \\
\hline 19 & UE-25 UZN \#23 & $\mathrm{M}^{*}$ & & & 54 & N54 4" Gage & $\mathbf{M}^{*}$ & & & 91 & UE25 WT-4 & $\mathrm{M}^{*}$ & & \\
\hline 20 & UE-25 UZN \#28 & $\mathbf{M}^{*}$ & & & 55 & USW UZ-N55 & $\mathrm{M}^{*}$ & & & 92 & UE25 WT-18 & $\mathbf{M}^{*}$ & & \\
\hline 21 & UE-25 UZN \#29 & $\mathbf{M}^{*}$ & & & 56 & USW UZ-N57 & $\mathbf{M}^{*}$ & & & 93 & USW H-5 & $\mathrm{M}^{*}$ & & \\
\hline 22 & UE-25 UZN \#30 & $\mathbf{M}^{*}$ & @ & & 57 & USW UZ-N64 & $\mathrm{M}^{*}$ & & & 94 & HRF Wedge & $\mathbf{M}^{*}$ & & \\
\hline
\end{tabular}


\$S Table 2. Summary of precipitation data from the nonrecording gage network at Yucca Mountain, Nevada, for water years 1992 and 1993 --Continued

\begin{tabular}{|c|c|c|c|c|c|c|c|c|c|c|c|c|c|}
\hline $\begin{array}{l}\text { Loca- } \\
\text { tion } \\
\text { code }\end{array}$ & $\begin{array}{c}\text { Site } \\
\text { nomenclature }\end{array}$ & $\begin{array}{l}\text { Amount } \\
\text { (Inches) }\end{array}$ & $\begin{array}{l}\text { Date } \\
\text { read }\end{array}$ & Notes & $\begin{array}{l}\text { Loca- } \\
\text { tlon } \\
\text { code }\end{array}$ & $\begin{array}{c}\text { Site } \\
\text { nomenclature }\end{array}$ & $\begin{array}{l}\text { Amount } \\
\text { (inches) }\end{array}$ & $\begin{array}{l}\text { Date } \\
\text { read }\end{array}$ & $\begin{array}{l}\text { Loca- } \\
\text { tion } \\
\text { code }\end{array}$ & $\begin{array}{c}\text { Site } \\
\text { nomenclature }\end{array}$ & $\begin{array}{l}\text { Amount } \\
\text { (inches) }\end{array}$ & $\begin{array}{l}\text { Date } \\
\text { read }\end{array}$ & Notes \\
\hline \multicolumn{14}{|c|}{ STORM OF FEBRUARY 18-19, 1993-Continued } \\
\hline 23 & UE-25 UZN \#56 & $\mathbf{M}^{*}$ & & & 58 & USW UZ-N65 & $\mathbf{M}^{*}$ & & 94 & HRF 4" Gage & $\mathbf{M}^{*}$ & & \\
\hline 24 & UE-25 UZN \#60 & $\mathbf{M}^{*}$ & & & 59 & USW UZ-N66 & $\mathbf{M}^{*}$ & & 94 & HRF 8" Gage & $M^{*}$ & & \\
\hline 25 & UE-25 UZN \#85 & $\mathbf{M}^{*}$ & & & 60 & USW UZ-N67 & $\mathbf{M}^{*}$ & & 95 & USW H-3 & $M^{*}$ & & \\
\hline 26 & UE-25 UZN \#92 & .55 & $2 / 22$ & & 60 & N67 4" Gage & $\mathbf{M}^{*}$ & & 96 & Wea station & $\mathbf{M}^{*}$ & & \\
\hline 26 & N92 4" Gage & .61 & $2 / 22$ & & 61 & USW UZ-N68 & $\mathbf{M}^{*}$ & & 97 & Wea station & $\mathbf{M}$ & & \\
\hline 27 & UE-25 UZN \#97 & $\mathbf{M}^{*}$ & & & 62 & USW UZ-N69 & $\mathbf{M}^{*}$ & & 98 & Evaporation & $\mathbf{M}^{*}$ & & \\
\hline 28 & UE-29 UZN \#91 & .60 & $2 / 22$ & & 63 & USW UZ-N70 & $\mathrm{M}^{*}$ & & 99 & Wea station & $\mathbf{M}^{*}$ & & \\
\hline 28 & N91 4" Gage & .68 & $2 / 22$ & & 63 & N70 4" Gage & $\mathbf{M}^{*}$ & & 99 & Wea station & $M^{*}$ & & \\
\hline 29 & USW UZ-N11 & $\mathbf{M}^{*}$ & & & 64 & USW UZ-N71 & $\mathbf{M}^{*}$ & & 100 & G-3 & $\mathbf{M}^{*}$ & & \\
\hline 29 & N11 4" Gage & $\mathbf{M}^{*}$ & & & 64 & N71 4" Gage & $\mathbf{M}^{*}$ & & 100 & G-3 8" Gage & $\mathbf{M}^{*}$ & & \\
\hline 30 & USW UZ-N15 & $\mathbf{M}^{*}$ & & & 65 & USW UZ-N72 & $\mathbf{M}^{*}$ & & 101 & Wea station & $\mathbf{M}^{*}$ & & \\
\hline 31 & USW UZ-N16 & $\mathbf{M}^{*}$ & & & 66 & USW UZ-N73 & $\mathbf{M}^{*}$ & & 101 & Wea station & $\mathbf{M}^{*}$ & & \\
\hline 32 & USW UZ-N17 & $\mathbf{M}^{*}$ & & & 67 & USW UZ-N74 & $\mathbf{M}^{*}$ & & 102 & Wea station & $\mathbf{M}^{*}$ & & \\
\hline 33 & USW UZ-N24 & $\mathbf{M}^{*}$ & & & 68 & USW UZ-N75 & $\mathbf{M}^{*}$ & & 103 & Wea station & .50 & $2 / 22$ & \\
\hline 33 & N24 4" Gage & NI & & & 68 & N75 4" Gage & $\mathbf{M}^{*}$ & & 103 & Wea station & .54 & $2 / 22$ & \\
\hline 34 & USW UZ-N25 & $\mathbf{M}^{*}$ & & & 69 & USW UZ-N76 & $\mathbf{M}^{*}$ & & 104 & Fran Ridge & $\mathbf{M}^{*}$ & & \\
\hline 35 & USW UZ-N26 & $\mathbf{M}^{*}$ & & & 70 & USW UZ-N77 & $\mathbf{M}^{*}$ & & 104 & Fran Ridge (8") & $\mathbf{M}^{*}$ & & \\
\hline 36 & USW UZ-N27 & $\mathbf{M}^{*}$ & & & 71 & USW UZ-N78 & $\mathbf{M}^{*}$ & & 105 & Plug Hill & $\mathbf{M}^{*}$ & & \\
\hline 36 & N27 8" Gage & $\mathbf{M}^{*}$ & & & 72 & USW UZ-N79 & $\mathbf{M}^{*}$ & & 106 & 4JA (8") & $\mathbf{M}^{*}$ & & \\
\hline \multirow[t]{2}{*}{37} & USW UZ-N35 & $\mathbf{M}^{*}$ & & & 73 & USW UZ-N80 & $\mathbf{M}^{*}$ & & 107 & Knothead Gap & .63 & $2 / 19$ & \\
\hline & & & & & & & & & 108 & Upper Forty Mile & $\mathbf{M}^{*}$ & & \\
\hline
\end{tabular}


Table 2. Summary of precipitation data from the nonrecording gage network at Yucca Mountain, Nevada, for water years 1992 and 1993 --Continued

\begin{tabular}{|c|c|c|c|c|c|c|c|c|c|c|c|c|c|c|}
\hline $\begin{array}{l}\text { Loca- } \\
\text { tlon } \\
\text { code }\end{array}$ & $\begin{array}{c}\text { Site } \\
\text { nomenclature }\end{array}$ & $\begin{array}{l}\text { Amount } \\
\text { (inches) }\end{array}$ & $\begin{array}{l}\text { Date } \\
\text { read }\end{array}$ & Notes & $\begin{array}{l}\text { Loca- } \\
\text { tion } \\
\text { code }\end{array}$ & $\begin{array}{c}\text { Site } \\
\text { nomenclature }\end{array}$ & $\begin{array}{l}\text { Amount } \\
\text { (inches) }\end{array}$ & $\begin{array}{l}\text { Date } \\
\text { read }\end{array}$ & Notes & $\begin{array}{l}\text { Loca- } \\
\text { tion } \\
\text { code }\end{array}$ & $\begin{array}{c}\text { Site } \\
\text { nomenclature }\end{array}$ & $\begin{array}{l}\text { Amount } \\
\text { (inches) }\end{array}$ & $\begin{array}{l}\text { Date } \\
\text { read }\end{array}$ & Notes \\
\hline \multicolumn{15}{|c|}{ STORM OF FEBRUARY 23, 1993} \\
\hline 1 & UE-25 UZN \#1 & .30 & $2 / 23$ & & 38 & USW UZ-N36 & .90 & $2 / 25$ & ** & 73 & N80 4" Gage & $\mathrm{M}^{*}$ & & \\
\hline 2 & UE-25 UZN \#2 & .31 & $2 / 23$ & & 39 & USW UZ-N37 & .82 & $2 / 23$ & ** & 74 & USW UZ-N81 & $\mathbf{M}^{*}$ & & \\
\hline 3 & UE-25 UZN \#3 & .31 & $2 / 23$ & & 39 & N37 4" Gage & .92 & $2 / 23$ & ** & 75 & USW UZ-N82 & $\mathbf{M}^{*}$ & & \\
\hline 4 & UE-25 UZN \#4 & .38 & $2 / 23$ & & 40 & USW UZ-N40 & .82 & $2 / 24$ & ** & 76 & USW UZ-N83 & $\mathbf{M}^{*}$ & & \\
\hline 4 & N4 4" Gage & .34 & $2 / 23$ & & 40 & N40 4" Gage & NI & & & 77 & USW UZ-N84 & $\mathbf{M}^{*}$ & & \\
\hline 5 & UE-25 UZN \#5 & .28 & $2 / 23$ & & 41 & USW UZ-N41 & .86 & $2 / 24$ & $* *$ & 78 & USW UZ-N86 & $\mathrm{M}^{*}$ & & \\
\hline 6 & UE-25 UZN \#6 & .29 & $2 / 23$ & & 42 & USW UZ-N42 & .84 & $2 / 24$ & ** & 79 & USW UZ-N87 & $\mathbf{M}^{*}$ & & \\
\hline 7 & UE-25 UZN \#7 & .32 & $2 / 23$ & & 43 & USW UZ-N43 & .84 & $2 / 24$ & ** & 80 & USW UZ-N88 & $\mathbf{M}^{*}$ & & \\
\hline 8 & UE-25 UZN \#8 & .30 & $2 / 23$ & & 43 & N43 4" Gage & NI & & & 81 & USW UZ-N89 & $\mathbf{M}^{*}$ & & \\
\hline 9 & UE-25 UZN \#9 & .34 & $2 / 23$ & & 44 & USW UZ-N44 & .92 & $2 / 24$ & @ 1 & 82 & USW UZ-N90 & $\mathbf{M}^{*}$ & & \\
\hline 10 & UE-25 UZN \#10 & .30 & $2 / 23$ & & 45 & USW UZ-N45 & .87 & $2 / 24$ & *** & 82 & N90 4" Gage & $\mathrm{M}^{*}$ & & \\
\hline 10 & N10 4" Gage & .28 & $2 / 23$ & & 46 & USW UZ-N46 & .27 & $2 / 24$ & & 83 & USW UZ-N93 & .77 & & \\
\hline 11 & UE-25 UZN \#12 & .34 & $2 / 23$ & & 47 & USW UZ-N47 & .24 & $2 / 24$ & & 84 & USW UZ-N94 & .75 & $2 / 25$ & ** \\
\hline 12 & UE-25 UZN $\# 13$ & .35 & $2 / 23$ & & 48 & USW UZ-N48 & .82 & $2 / 24$ & *** & 85 & USW UZ-N95 & .69 & $2 / 25$ & \\
\hline 13 & UE-25 UZN \#14 & .31 & $2 / 23$ & & 49 & USW UZ-N49 & .70 & $2 / 24$ & $* *$ & 86 & USW UZ-N96 & .56 & $2 / 25$ & \\
\hline 14 & UE-25 UZN \#18 & .80 & $2 / 23$ & $* *$ & 50 & USW UZ-N50 & .75 & $2 / 24$ & $* *$ & 87 & USW UZ-N98 & .82 & & \\
\hline 15 & UE-25 UZN \#19 & .94 & $2 / 24$ & $* *$ & 50 & N50 4" Gage & NI & & & 87 & N98 4" Gage & NI & & \\
\hline 16 & UE-25 UZN \#20 & .84 & $2 / 24$ & $* *$ & 51 & USW UZ-N51 & .88 & $2 / 24$ & $* *$ & 88 & USW UZ-13 & .57 & & \\
\hline 17 & UE-25 UZN \#21 & .84 & $2 / 24$ & $* *$ & 52 & USW UZ-N52 & .75 & $2 / 24$ & ** & 88 & UZ-13 4" Gage & .32 & & \\
\hline 17 & N21 4" Gage & NI & & & 53 & USW UZ-N53 & .82 & $2 / 24$ & ** & 89 & USW GA-1 & .82 & $2 / 25$ & ** \\
\hline 18 & UE-25 UZN $\# 22$ & .89 & $2 / 24$ & *** & 54 & USW UZ-N54 & .80 & $2 / 24$ & ** & 90 & USW G-2 & .83 & & \\
\hline 19 & UE-25 UZN \#23 & .82 & $2 / 24$ & ** & 54 & N54 4" Gage & .86 & $2 / 24$ & $* *$ & 91 & UE25 WT-4 & .79 & & \\
\hline 20 & UE-25 UZN \#28 & .80 & $2 / 24$ & ** & 55 & USW UZ-N55 & .75 & $2 / 24$ & $* *$ & 92 & UE25 WT-18 & .81 & & \\
\hline 21 & UE-25 UZN \#29 & .80 & $2 / 24$ & ** & 56 & USW UZ-N57 & .79 & $2 / 25$ & ** & 93 & USW H-5 & .69 & & \\
\hline 22 & UE-25 UZN \#30 & .90 & $2 / 24$ & *** & 57 & USW UZ-N64 & .76 & $2 / 25$ & $* *$ & 94 & HRF Wedge & 1.13 & & \\
\hline
\end{tabular}




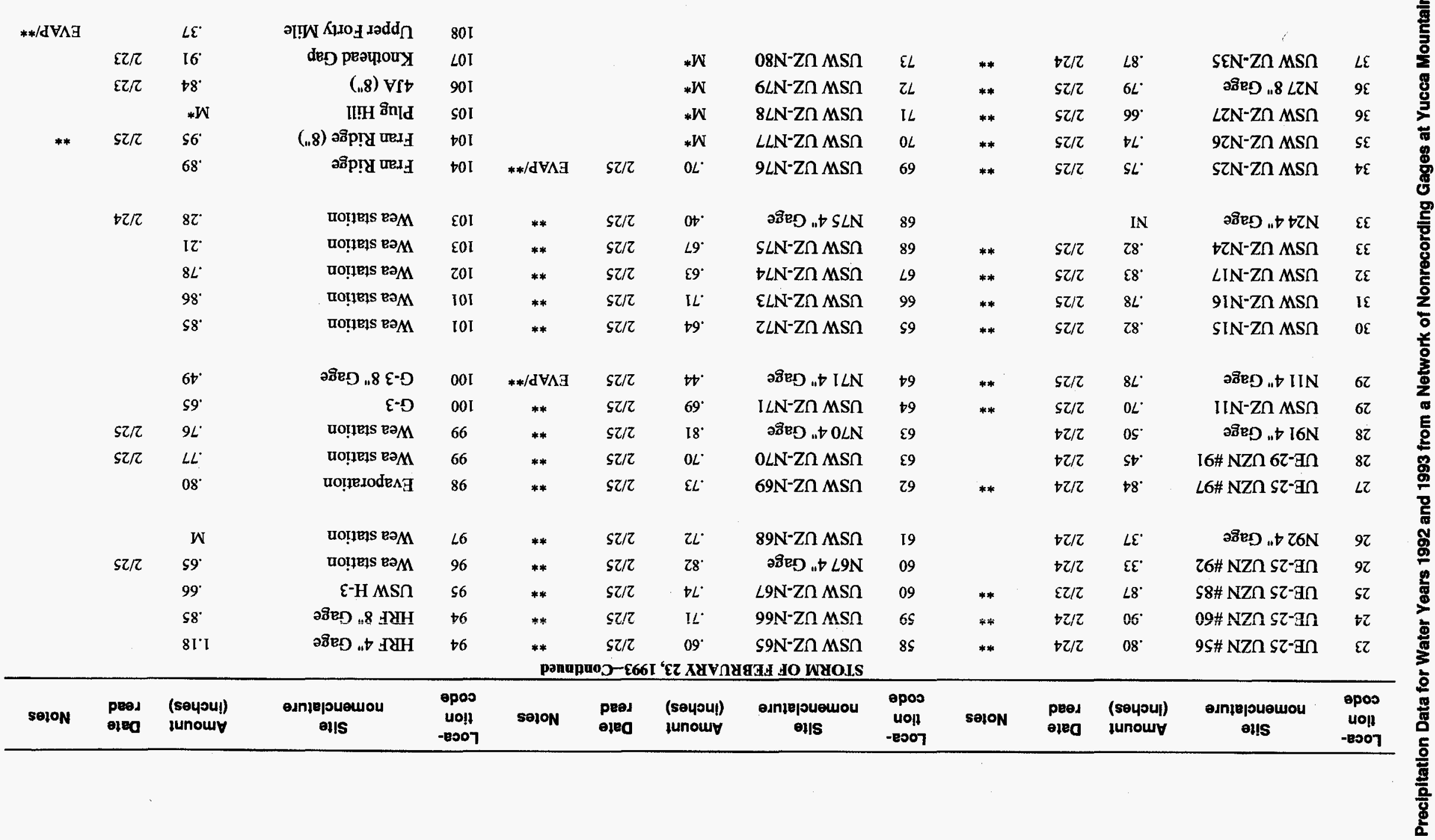


Table 2. Summary of precipitation data from the nonrecording gage network at Yucca Mountain, Nevada, for water years 1992 and 1993--Continued

\begin{tabular}{|c|c|c|c|c|c|c|c|c|c|c|c|c|c|c|}
\hline $\begin{array}{l}\text { Loca- } \\
\text { tion } \\
\text { code }\end{array}$ & $\begin{array}{c}\text { Site } \\
\text { nomenclature }\end{array}$ & $\begin{array}{l}\text { Amount } \\
\text { (inches) }\end{array}$ & $\begin{array}{l}\text { Date } \\
\text { read }\end{array}$ & Notes & $\begin{array}{l}\text { Loca- } \\
\text { tion } \\
\text { code }\end{array}$ & $\begin{array}{c}\text { Site } \\
\text { nomenclature }\end{array}$ & $\begin{array}{l}\text { Amount } \\
\text { (inches) }\end{array}$ & $\begin{array}{l}\text { Date } \\
\text { read }\end{array}$ & Notes & $\begin{array}{l}\text { Loca- } \\
\text { tion } \\
\text { code }\end{array}$ & $\begin{array}{c}\text { Slie } \\
\text { nomenclature }\end{array}$ & $\begin{array}{l}\text { Amount } \\
\text { (Inches) }\end{array}$ & $\begin{array}{l}\text { Date } \\
\text { read }\end{array}$ & Notes \\
\hline \multicolumn{15}{|c|}{ STORM OF FEBRUARY 26-28, 1993} \\
\hline 1 & UE-25 UZN \#1 & .96 & $3 / 1$ & & 38 & USW UZ-N36 & .59 & $3 / 12$ & sNow & 73 & N80 4" Gage & 1.72 & $3 / 12$ & \\
\hline 2 & UE-25 UZN \#2 & 1.00 & $3 / 1$ & & 39 & USW UZ-N37 & .93 & $3 / 12$ & & 74 & USW UZ-N81 & 1.70 & $3 / 12$ & $* *$ \\
\hline 3 & UE-25 UZN \#3 & .89 & $3 / 1$ & & 39 & N37 4" Gage & .95 & $3 / 12$ & & 75 & USW UZ-N82 & 1.62 & $3 / 12$ & ** \\
\hline 4 & UE-25 UZN \#4 & .93 & $3 / 1$ & & 40 & USW UZ-N40 & .93 & $3 / 8$ & & 76 & USW UZ-N83 & 1.75 & $3 / 12$ & ** \\
\hline 4 & N4 4" Gage & .91 & $3 / 1$ & & 40 & N40 4" Gage & NI & & & 77 & USW UZ-N84 & 1.55 & $3 / 12$ & \\
\hline 5 & UE-25 UZN \#5 & .90 & $3 / 1$ & & 41 & USW UZ-N4I & .99 & $3 / 8$ & & 78 & USW UZ-N86 & 1.75 & $3 / 12$ & ** \\
\hline 6 & UE-25 UZN \#6 & .92 & $3 / 1$ & & 42 & USW UZ-N42 & .91 & $3 / 8$ & & 79 & USW UZ-N87 & 1.65 & $3 / 12$ & ** \\
\hline 7 & UE-25 UZN \#7 & .95 & $3 / 1$ & & 43 & USW UZ-N43 & .91 & $3 / 8$ & & 80 & USW UZ-N88 & 1.48 & $3 / 12$ & $* *$ \\
\hline 8 & UE-25 UZN \#8 & .86 & $3 / 1$ & & 43 & N43 4" Gage & NI & & & 81 & USW UZ-N89 & 1.55 & $3 / 12$ & ** \\
\hline 9 & UE-25 UZN \#9 & .95 & $3 / 1$ & & 44 & USW UZ-N44 & .95 & $3 / 8$ & & 82 & USW UZ-N90 & 1.55 & $3 / 12$ & ** \\
\hline 10 & UE-25 UZN \#10 & .93 & $3 / 1$ & & 45 & USW UZ-N45 & 1.00 & $3 / 8$ & & 82 & N90 4" Gage & 1.62 & $3 / 12$ & ** \\
\hline 10 & N10 4" Gage & .90 & $3 / 1$ & & 46 & USW UZ-N46 & .83 & $3 / 1$ & & 83 & USW UZ-N93 & .55 & & \\
\hline 11 & UE-25 UZN \#12 & .87 & $3 / 1$ & & 47 & USW UZ-N47 & .84 & $3 / 1$ & & 84 & USW UZ-N94 & .62 & $3 / 1$ & SNOW \\
\hline 12 & UE-25 UZN \#13 & .94 & $3 / 12$ & & 48 & USW UZ-N48 & .98 & $3 / 6$ & & 85 & USW UZ-N95 & .80 & $3 / 1$ & SNOW \\
\hline 13 & UE-25 UZN \#14 & .89 & $3 / 12$ & & 49 & USW UZ-N49 & 1.00 & $3 / 6$ & & 86 & USW UZ-N96 & .55 & $3 / 1$ & SNOW \\
\hline 14 & UE-25 UZN \#18 & .95 & $3 / 1$ & & 50 & USW UZ-N50 & 1.00 & $3 / 6$ & & 87 & USW UZ-N98 & .98 & $3 / 17$ & EVAP \\
\hline 15 & UE-25 UZN \#19 & 1.00 & $3 / 17$ & & 50 & N50 4" Gage & $\mathrm{NI}$ & & & 87 & N98 4" Gage & Ní & & \\
\hline 16 & UE-25 UZN \#20 & .96 & $3 / 17$ & EVAP & 51 & USW UZ-N51 & .96 & $3 / 6$ & & 88 & USW UZ-13 & .34 & $3 / 1$ & \\
\hline 17 & UE-25 UZN \#21 & .94 & $3 / 17$ & EVAP & 52 & USW UZ-N52 & .95 & $3 / 6$ & & 88 & UZ-13 4" Gage & .50 & $3 / 1$ & \\
\hline 17 & N21 4" Gage & NI & & & 53 & USW UZ-N53 & .93 & $3 / 10$ & & 89 & USW GA-1 & .41 & $3 / 12$ & SNOW \\
\hline 18 & UE-25 UZN \#22 & 1.02 & $3 / 17$ & EVAP & 54 & USW UZ-N54 & .88 & $3 / 12$ & & 90 & USW G-2 & .39 & $3 / 12$ & \\
\hline 19 & UE-25 UZN \#23 & .93 & $3 / 17$ & EVAP & 54 & N54 4" Gage & .95 & $3 / 12$ & & 91 & UE25 WT-4 & .81 & $3 / 12$ & \\
\hline 20 & UE-25 UZN \#28 & .95 & $3 / 17$ & EVAP & 55 & USW UZ-N55 & .96 & $3 / 12$ & & 92 & UE25 WT-18 & .64 & $3 / 12$ & \\
\hline 21 & UE-25 UZN \#29 & .95 & $3 / 17$ & EVAP & 56 & USW UZ-N57 & .89 & $3 / 1$ & SNOW & 93 & USW H-5 & .49 & & \\
\hline 22 & UE-25 UZN \#30 & 1.05 & $3 / 17$ & EVAP & 57 & USW UZ-N64 & .64 & $3 / 1$ & SNOW & 94 & HRF Wedge & .60 & & \\
\hline
\end{tabular}


8 Table 2. Summary of precipitation data from the nonrecording gage network at Yucca Mountain, Nevada, for water years 1992 and 1993 --Continued

\begin{tabular}{|c|c|c|c|c|c|c|c|c|c|c|c|c|c|c|}
\hline $\begin{array}{l}\text { Loca- } \\
\text { tion } \\
\text { code }\end{array}$ & $\begin{array}{c}\text { Site } \\
\text { nomenclature }\end{array}$ & $\begin{array}{l}\text { Amount } \\
\text { (inches) }\end{array}$ & $\begin{array}{l}\text { Date } \\
\text { read }\end{array}$ & Notes & $\begin{array}{l}\text { Loca- } \\
\text { tion } \\
\text { code }\end{array}$ & $\begin{array}{c}\text { Site } \\
\text { nomenclature }\end{array}$ & $\begin{array}{l}\text { Amount } \\
\text { (Inches) }\end{array}$ & $\begin{array}{l}\text { Date } \\
\text { read }\end{array}$ & Notes & $\begin{array}{l}\text { Loce- } \\
\text { tion } \\
\text { code }\end{array}$ & $\begin{array}{c}\text { Site } \\
\text { nomenclature }\end{array}$ & $\begin{array}{l}\text { Amount } \\
\text { (inches) }\end{array}$ & $\begin{array}{l}\text { Date } \\
\text { read }\end{array}$ & Notes \\
\hline \multicolumn{15}{|c|}{ STORM OF FEBRUARY 26-28, 1993-Continued } \\
\hline 23 & UE-25 UZN \#56 & 1.00 & $3 / 6$ & & 58 & USW UZ-N65 & .85 & $3 / 1$ & & 94 & HRF 4" Gage & .70 & $3 / 1$ & \\
\hline 24 & UE-25 UZN \#60 & 1.02 & $3 / 12$ & & 59 & USW UZ-NNó6 & .79 & $3 / 1$ & & 94 & HRF 8" Gage & .70 & $3 / 1$ & \\
\hline 25 & UE-25 UZN \#85 & .85 & $3 / 1$ & & 60 & USW UZ-N67 & .91 & $3 / 1$ & & 95 & USW H-3 & .28 & $3 / 1$ & SNOW \\
\hline 26 & UE-25 UZN \#92 & .50 & $3 / 1$ & & 60 & N67 4" Gage & .98 & $3 / 1$ & & 96 & Wea station & .49 & $3 / 1$ & SNOW \\
\hline 26 & N92 4" Gage & .53 & $3 / 1$ & & 61 & USW UZ-N68 & .98 & $3 / 1$ & & 97 & Wea station & $\mathbf{M}$ & & \\
\hline 27 & UE-25 UZN \#97 & 1.02 & $3 / 17$ & & 62 & USW UZ-N69 & .93 & $3 / 1$ & & 98 & Evaporation & .66 & $3 / 12$ & \\
\hline 28 & UE-29 UZN \#91 & .50 & $3 / 1$ & & 63 & USW UZ-N70 & .91 & $3 / 17$ & EVAP & 99 & Wea station & .40 & $3 / 1$ & \\
\hline 28 & N91 4" Gage & .59 & $3 / 1$ & & 63 & N70 4" Gage & .85 & $3 / 17$ & EVAP & 99 & Wea station & .52 & $3 / 1$ & \\
\hline 29 & USW UZ-N11 & .42 & $3 / 12$ & SNOW & 64 & USW UZ-N71 & .56 & $3 / 1$ & SNOW & 100 & $\mathrm{G}-3$ & .57 & & \\
\hline 29 & N11 4" Gage & .38 & $3 / 12$ & SNOW & 64 & N71 4" Gage & .45 & $3 / 1$ & SNOW & 100 & G-3 8" Gage & .80 & & \\
\hline 30 & USW UZ-N15 & .51 & $3 / 12$ & SNOW & 65 & USW UZ-N72 & .51 & $3 / 1$ & SNOW & 101 & Wea station & 1.00 & & \\
\hline 31 & USW UZ-N16 & .50 & $3 / 12$ & SNOW & 66 & USW UZ-N73 & .54 & $3 / 1$ & SNOW & 101 & Wea station & 1.00 & & \\
\hline 32 & USW UZ-N17 & .44 & $3 / 12$ & SNOW & 67 & USW UZ-N74 & .57 & $3 / 1$ & SNOW & 102 & Wea station & .65 & & \\
\hline 33 & USW UZ-N24 & .93 & $3 / 17$ & EVAP & 68 & USW UZ-N75 & .56 & $3 / 1$ & SNOW & 103 & Wea station & .80 & & \\
\hline 33 & N24 4" Gage & NI & & & 68 & N75 4" Gage & .59 & $3 / 1$ & SNOW & 103 & Wea station & .94 & $3 / 1$ & SNOW \\
\hline 34 & USW UZ-N25 & .95 & $3 / 17$ & EVAP & 69 & USW UZ-N76 & .55 & $3 / 1$ & SNOW & 104 & Fran Ridge & .71 & & \\
\hline 35 & USW UZ-N26 & .94 & $3 / 17$ & EVAP & 70 & USW UZ-N77 & 1.60 & $3 / 9$ & $* *$ & 104 & Fran Ridge ( $\left.8^{\prime \prime}\right)$ & .87 & & \\
\hline 36 & USW UZ-N27 & .68 & $3 / 1$ & SNOW & 71 & USW UZ-N78 & 1.50 & $3 / 12$ & $m *$ & 105 & Plug Hill & 1.25 & & \\
\hline 36 & N27 8" Gage & .72 & $3 / 1$ & SNOW & 72 & USW UZ-N79 & 1.70 & $3 / 12$ & $* *$ & 106 & 4JA (8") & & & \\
\hline \multirow[t]{2}{*}{37} & USW UZ-N35 & 1.02 & $3 / 12$ & & 73 & USW UZ-N80 & 1.60 & $3 / 12$ & $* *$ & 107 & Knothead Gap & 1.02 & $3 / 12$ & \\
\hline & & & & & & & & & & 108 & Upper Forty Mile & .54 & $3 / 1$ & \\
\hline
\end{tabular}


Table 2. Summary of precipitation data from the nonrecording gage network at Yucca Mountain, Nevada, for water years 1992 and $1993-$ Continued

\begin{tabular}{|c|c|c|c|c|c|c|c|c|c|c|c|c|c|c|}
\hline $\begin{array}{l}\text { Loca- } \\
\text { tion } \\
\text { code }\end{array}$ & $\begin{array}{c}\text { Site } \\
\text { nomenclature }\end{array}$ & $\begin{array}{l}\text { Amount } \\
\text { (Inches) }\end{array}$ & $\begin{array}{l}\text { Date } \\
\text { read }\end{array}$ & Notes & $\begin{array}{l}\text { Loca- } \\
\text { tion } \\
\text { code }\end{array}$ & $\begin{array}{c}\text { Site } \\
\text { nomenclature }\end{array}$ & $\begin{array}{l}\text { Amount } \\
\text { (Inches) }\end{array}$ & $\begin{array}{l}\text { Date } \\
\text { read }\end{array}$ & Notes & $\begin{array}{l}\text { Loca- } \\
\text { tion } \\
\text { code }\end{array}$ & $\begin{array}{c}\text { Site } \\
\text { nomenelature }\end{array}$ & $\begin{array}{l}\text { Amount } \\
\text { (inches) }\end{array}$ & $\begin{array}{l}\text { Date } \\
\text { read }\end{array}$ & Notes \\
\hline \multicolumn{15}{|c|}{ STORM OF MARCH 26-28, 1993} \\
\hline 1 & UE-25 UZN \#1 & .94 & $3 / 30$ & & 38 & USW UZ-N36 & 1.00 & $3 / 29$ & & 73 & N80 4" Gage & 1.10 & $3 / 29$ & \\
\hline 2 & UE-25 UZN \#2 & .94 & $3 / 30$ & & 39 & USW UZ-N37 & .85 & $3 / 29$ & & 74 & USW UZ-N81 & .95 & $3 / 29$ & \\
\hline 3 & UE-25 UZN \#3 & .85 & $3 / 30$ & & 39 & N37 4" Gage & .94 & $3 / 30$ & & 75 & USW UZ-N82 & .87 & $3 / 29$ & \\
\hline 4 & UE-25 UZN \#4 & .90 & $3 / 30$ & & 40 & USW UZ-N40 & .90 & $3 / 30$ & & 76 & USW UZ-N83 & .98 & $3 / 29$ & \\
\hline 4 & N4 4" Gage & & & Removed & 40 & N40 4" Gage & NI & & & 77 & USW UZ-N84 & .99 & $3 / 29$ & \\
\hline 5 & UE-25 UZN \#5 & .85 & $3 / 30$ & & 41 & USW UZ-N41 & .95 & $3 / 30$ & & 78 & USW UZ-N86 & 1.05 & $3 / 29$ & \\
\hline 6 & UE-25 UZN \#6 & .80 & $3 / 30$ & & 42 & USW UZ-N42 & .92 & $3 / 30$ & & 79 & USW UZ-N87 & .97 & $3 / 29$ & \\
\hline 7 & UE-25 UZN \#7 & .95 & $3 / 30$ & & 43 & USW UZ-N43 & .90 & $3 / 30$ & & 80 & USW UZ-N88 & 1.08 & $3 / 29$ & \\
\hline 8 & UE-25 UZN \#8 & .90 & $3 / 30$ & & 43 & N43 4" Gage & NI & & & 81 & USW UZ-N89 & .97 & $3 / 29$ & \\
\hline 9 & UE-25 UZN \#9 & .92 & $3 / 30$ & & 44 & USW UZ-N44 & .95 & $3 / 30$ & & 82 & USW UZ-N90 & .93 & $3 / 29$ & \\
\hline 10 & UE-25 UZN \#10 & .96 & $3 / 30$ & & 45 & USW UZ-N45 & .99 & $3 / 30$ & & 82 & N90 4" Gage & 1.08 & $3 / 29$ & \\
\hline 10 & N10 4" Gage & & & Removed & 46 & USW UZ-N46 & 1.01 & $3 / 30$ & & 83 & USW UZ-N93 & .83 & $4 / 1$ & \\
\hline 11 & UE-25 UZN \#12 & .85 & $3 / 30$ & & 47 & USW UZ-N47 & .90 & $3 / 30$ & & 84 & USW UZ-N94 & .80 & $4 / 1$ & \\
\hline 12 & UE-25 UZN \#13 & .92 & $3 / 30$ & & 48 & USW UZ-N48 & .91 & $3 / 30$ & & 85 & USW UZ-N95 & .80 & $4 / 1$ & \\
\hline 13 & UE-25 UZN \#14 & .80 & $3 / 30$ & & 49 & USW UZ-N49 & .90 & $3 / 30$ & & 86 & USW UZ-N96 & .75 & $4 / 1$ & \\
\hline 14 & UE-25 UZN \#18 & .82 & $3 / 30$ & & 50 & USW UZ-N50 & .89 & $3 / 30$ & & 87 & USW UZ-N98 & .93 & $4 / 1$ & \\
\hline 15 & UE-25 UZN \#19 & .92 & $3 / 30$ & & 50 & N50 4" Gage & NI & & & 87 & N98 4" Gage & NI & & \\
\hline 16 & UE-25 UZN \#20 & .88 & $3 / 30$ & & 51 & USW UZ-N51 & .97 & $3 / 30$ & & 88 & USW UZ 13 & .83 & $4 / 1$ & \\
\hline 17 & UE-25 UZN \#21 & .85 & $3 / 30$ & & 52 & USW UZ-N52 & .86 & $3 / 30$ & & 88 & UZ-13 4" Gage & .90 & $4 / 1$ & \\
\hline 17 & N21 4" Gage & NI & & & 53 & USW UZ-N53 & .86 & $3 / 30$ & & 89 & USW GA-1 & .89 & $4 / 2$ & SNOW \\
\hline 18 & UE-25 UZN \#22 & .90 & $3 / 30$ & & 54 & USW UZ-N54 & .82 & $3 / 30$ & & 90 & USW G-2 & .92 & $4 / 2$ & SNOW \\
\hline 19 & UE-25 UZN \#23 & .90 & $3 / 30$ & & 54 & N54 4" Gage & .93 & $3 / 30$ & & 91 & UE25 WT-4 & .83 & $3 / 31$ & \\
\hline 20 & UE-25 UZN \#28 & .90 & $3 / 30$ & & 55 & USW UZ-N55 & .88 & $3 / 30$ & & 92 & UE25 WT-18 & .97 & $4 / 2$ & \\
\hline 21 & UE-25 UZN \#29 & .87 & $3 / 30$ & & 56 & USW UZ-N57 & 1.07 & $4 / 1$ & & 93 & USW H-5 & .71 & $4 / 1$ & \\
\hline 22 & UE-25 UZN \#30 & .99 & $3 / 30$ & & 57 & USW UZ-N64 & .88 & $4 / 1$ & & 94 & HRF Wedge & .60 & $3 / 29$ & \\
\hline
\end{tabular}




\begin{tabular}{|c|c|c|c|c|c|c|c|c|c|c|c|c|c|c|}
\hline & $6 z / \mathfrak{\varepsilon}$ & $60^{\circ} \mathrm{I}$ & 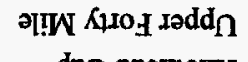 & 801 & & & & & & & & & & \\
\hline & $\mathfrak{I E} / \mathcal{E}$ & $z 8^{\prime}$ & deD peaqloty & LOI & & $6 Z / \mathfrak{E}$ & $\angle O^{\circ} I$ & 08N-Zn MSח & $\varepsilon L$ & & $z / t$ & $66^{\circ}$ & SEN-Zn MSก & $L \mathcal{E}$ \\
\hline & $0 \varepsilon / \varepsilon$ & $\varepsilon L^{\circ}$ & (..8) VIt & $90 \mathrm{I}$ & & $6 Z / \mathcal{E}$ & $00^{\prime} \mathrm{I}$ & $6 \angle N-Z \cap M S \cap$ & $\tau L$ & & $\mathrm{I} / \mathrm{t}$ & $16^{\circ}$ & 98RD „ , 8 LZN & $9 \varepsilon$ \\
\hline & $6 \tau / \mathfrak{\varepsilon}$ & IL' & II!H ${ }^{8 n}[d$ & SOI & & $6 z / \varepsilon$ & $s 6^{\circ}$ & $8 L N-Z n M S \cap$ & IL & & $I / t$ & $\varepsilon 8^{\circ}$ & $\angle Z N-Z \cap M S \Omega$ & $9 \mathcal{E}$ \\
\hline \multirow[t]{17}{*}{$d \forall \Lambda \exists$} & $I \varepsilon / \varepsilon$ & $08^{\circ}$ & 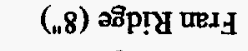 & t0I & & $6 z / \varepsilon$ & $\angle 8^{\circ}$ & LLN-Zn MS & $0 L$ & & $1 \varepsilon / \mathcal{E}$ & $68^{\circ}$ & $9 \mathrm{ZN}-\mathrm{Zn} \mathrm{MS} \Omega$ & $\mathfrak{s}$ \\
\hline & $\mathfrak{I \varepsilon / \varepsilon}$ & $\$ 8^{\circ}$ & 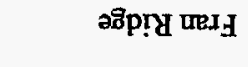 & toI & & $\mathrm{I} / \mathrm{t}$ & 0 & $9 L N-Z \cap M S \cap$ & 69 & & $\mathfrak{T \varepsilon / \varepsilon}$ & $\varepsilon 6^{\circ}$ & SZN-Zn MSn & $t \varepsilon$ \\
\hline & $\mathrm{I} \varepsilon / \mathcal{E}$ & $\varepsilon 6^{\circ}$ & นо!̣E१S вәM & E0t & & $1 / t$ & W & วฮิะD „ † SLN & 89 & & & IN & 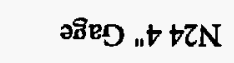 & $\mathfrak{E}$ \\
\hline & IE/E & $68^{\circ}$ & 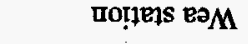 & E0I & & $\mathrm{I} / \mathrm{t}$ & $08^{\circ}$ & SLN-Zn MSก & 89 & & $I \varepsilon / \varepsilon$ & $\$ 6^{\circ}$ & $\forall Z N-Z \cap M S \cap$ & $\mathfrak{\varepsilon}$ \\
\hline & $0 \mathcal{E} / \mathfrak{E}$ & $\$ L$ & uo!̣e1S EOM & zoI & & $\mathrm{I} / \mathrm{t}$ & $O L^{\circ}$ & $t \angle N-Z \cap M S \cap$ & $\angle 9$ & MONS & $z / t$ & $26^{\circ}$ & LIN-Zn MSO & $\tau \varepsilon$ \\
\hline & $0 \varepsilon / \varepsilon$ & $88^{\circ}$ & पо!̣е१S вәM & I0I & & $\mathrm{I} / \mathrm{t}$ & $+8^{\circ}$ & $\varepsilon \angle N-Z \cap M S \cap$ & 99 & MONS & $i / /$ & $26^{\circ}$ & $9 \mathrm{IN}-\mathrm{Zn} \mathrm{MS} \Omega$ & I⿷ \\
\hline & $0 \varepsilon / \varepsilon$ & $\angle 8^{\circ}$ & पOIIETS ROM & tot & & $\mathrm{I} / \mathrm{t}$ & $\angle 9^{\circ}$ & $\tau \angle N-Z \cap M S \Omega$ & $\varsigma 9$ & MONS & $z / t$ & 16 & SIN-Zn MSR & $0 \varepsilon$ \\
\hline & $1 / t$ & $\varepsilon 0^{\circ} \mathrm{I}$ & วฮิย „8 $8-0$ & 00I & & $1 / t$ & $\angle L^{\circ}$ & aชిED . b ILN & t9 & MONS & $z / t$ & $6 L^{\circ}$ & วฮีषD „. IIN & 62 \\
\hline & $1 / t$ & $\mathfrak{\varepsilon} 6^{\circ}$ & $\varepsilon-D$ & 00I & & $\mathrm{I} / \mathrm{t}$ & SL & $\mathrm{I} \angle \mathrm{N}-\mathrm{Z} \cap \mathrm{MS} \Omega$ & t9 & MONS & $2 / t$ & $\varsigma L^{\circ}$ & IIN-Zn MSn & 62 \\
\hline & $\mathrm{I} / \mathrm{t}$ & $\varepsilon 6^{\circ}$ & UO!IEIS EәM & 66 & & I $\varepsilon / \mathcal{E}$ & 96 & aสైอ „ & $\varepsilon 9$ & & $6 z / \varepsilon$ & $80^{\circ} \tau$ & ว88D „ t I6N & 82 \\
\hline & $1 / t$ & $98^{\circ}$ & 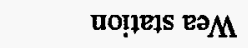 & 66 & & $I \varepsilon / \mathcal{E}$ & $\angle 8^{\circ}$ & $0 L N-Z \cap M S \cap$ & E9 & & $6 Z / \varepsilon$ & $09^{\circ}$ & I6\# NZח 62-3ก & 82 \\
\hline & $0 \varepsilon / \varepsilon$ & $O L^{\circ}$ & uọ̣exodeng & 86 & (0) & $\mathrm{I} / \mathrm{t}$ & $\angle 8$ & $69 \mathrm{~N}-\mathrm{Zn} \mathrm{MS} \Omega$ & 29 & & $0 \varepsilon / \varepsilon$ & $\varepsilon 6^{\circ}$ & L6\#NZП sZ-G & $\angle z$ \\
\hline & & W & uonjeis eəM & $L 6$ & & $\mathrm{I} / \mathrm{t}$ & 18 & 89N-Zn MSח & 19 & & $6 z / \varepsilon$ & $\angle I^{\prime} I$ & a8ed „. Z Z6N & 92 \\
\hline & $\mathrm{L} / \mathrm{t}$ & $0 L^{\circ}$ & uo!̣EIS вәM & 96 & & $\mathrm{I} / \mathrm{t}$ & 26 & 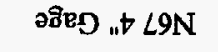 & 09 & & $6 Z / \mathcal{E}$ & $20^{\circ} \mathrm{I}$ & Z6\# NZก sZ-8ก & $9 z$ \\
\hline & $I / t$ & $\varepsilon L^{\circ}$ & $\varepsilon-H M S \cap$ & \$6 & & $I / t$ & $\$ 8^{\circ}$ & $\angle 9 N-Z \cap M S \cap$ & 09 & & $i / t$ & $L^{\circ}$ & 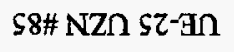 & $\varsigma z$ \\
\hline & $6 \tau / \varepsilon$ & $0 L^{\circ}$ & 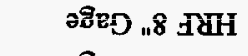 & $i 6$ & (0) & $\mathrm{I} / \mathrm{t}$ & $\$ 6$ & $99 \mathrm{~N}^{-} \mathrm{Z} \cap \mathrm{MS} \Omega$ & $6 S$ & & $0 \varepsilon / \varepsilon$ & $06^{\circ}$ & 09\# NZח sZ-3n & $t z$ \\
\hline & $6 \tau / \mathfrak{\varepsilon}$ & $Z L^{\circ}$ & ә8ิ80 „. & t6 & pont & $\frac{1 / t}{105-\varepsilon 6}$ & $\begin{array}{c}S L ' \\
' 8 z-9 \tau \text { HO }\end{array}$ & 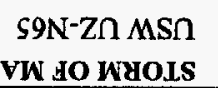 & $8 \varsigma$ & & $0 \mathcal{E} / \mathcal{E}$ & I8. & 9S\# NZn ऽZ-An & $\varepsilon z$ \\
\hline solon & $\begin{array}{l}\text { peed } \\
\text { eleo }\end{array}$ & $\begin{array}{l}\text { (seyou) } \\
\text { junowy }\end{array}$ & $\begin{array}{l}\text { esnje|puowou } \\
\text { elIs }\end{array}$ & $\begin{array}{l}\text { opos } \\
\text { แo!l } \\
-8007\end{array}$ & selon & $\begin{array}{l}\text { peed } \\
\text { oleo }\end{array}$ & $\begin{array}{l}\text { (soysul) } \\
\text { lunowy }\end{array}$ & $\begin{array}{l}\text { Odniepuewou } \\
\text { elIS }\end{array}$ & $\begin{array}{c}\text { epos } \\
\text { uolp } \\
-8007\end{array}$ & selon & $\begin{array}{l}\text { peed } \\
\text { elea }\end{array}$ & $\begin{array}{l}\text { (soupul) } \\
\text { funowy }\end{array}$ & 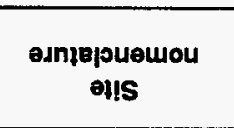 & $\begin{array}{l}\text { opos } \\
\text { Lo!l } \\
-8007\end{array}$ \\
\hline
\end{tabular}


Table 2. Summary of precipitation data from the nonrecording gage network at Yucca Mountain, Nevada, for water years 1992 and $1993--$ Continued

\begin{tabular}{|c|c|c|c|c|c|c|c|c|c|c|c|c|c|c|}
\hline $\begin{array}{l}\text { Loca- } \\
\text { tlon } \\
\text { code }\end{array}$ & $\begin{array}{c}\text { Site } \\
\text { nomenclature }\end{array}$ & $\begin{array}{l}\text { Amount } \\
\text { (inches) }\end{array}$ & $\begin{array}{l}\text { Date } \\
\text { read }\end{array}$ & Notes & $\begin{array}{l}\text { Loca- } \\
\text { tion } \\
\text { code }\end{array}$ & $\begin{array}{c}\text { Site } \\
\text { nomenclature }\end{array}$ & $\begin{array}{l}\text { Amount } \\
\text { (inches) }\end{array}$ & $\begin{array}{l}\text { Date } \\
\text { read }\end{array}$ & Notes & $\begin{array}{l}\text { Loca- } \\
\text { tion } \\
\text { code }\end{array}$ & $\begin{array}{c}\text { Site } \\
\text { nomenclature }\end{array}$ & $\begin{array}{l}\text { Amount } \\
\text { (inches) }\end{array}$ & $\begin{array}{l}\text { Date } \\
\text { read }\end{array}$ & Notes \\
\hline \multicolumn{15}{|c|}{ STORM OF JUNE 5-6, 1993} \\
\hline 1 & UE-25 UZN \#1 & .57 & $6 / 9$ & & 38 & USW UZ-N36 & 86 & $6 / 9$ & & 73 & N80 4" Gage & .73 & $6 / 9$ & \\
\hline 2 & UE-25 UZN \#2 & .57 & $6 / 9$ & & 39 & USW UZ-N37 & .61 & $6 / 9$ & & 74 & USW UZ-N81 & .67 & $6 / 9$ & \\
\hline 3 & UE-25 UZN \#3 & .55 & $6 / 9$ & & 39 & N37 4" Gage & .67 & $6 / 9$ & & 75 & USW UZ-N82 & .69 & $6 / 9$ & \\
\hline 4 & UE-25 UZN \#4 & .62 & $6 / 9$ & & 40 & USW UZ-N40 & .55 & $6 / 8$ & & 76 & USW UZ-N83 & $\mathrm{M}$ & $6 / 9$ & \\
\hline 4 & N4 4" Gage & & & Removed & 40 & N40 4" Gage & NI & & & 77 & USW UZ-N84 & .71 & $6 / 9$ & \\
\hline 5 & UE-25 UZN \#5 & .55 & $6 / 9$ & & 41 & USW UZ-N4I & .62 & $6 / 8$ & & 78 & USW UZ-N86 & .75 & $6 / 9$ & \\
\hline 6 & UE-25 UZN \#6 & .54 & $6 / 9$ & & 42 & USW UZ-N42 & .58 & $6 / 8$ & & 79 & USW UZ-N87 & .69 & $6 / 9$ & \\
\hline 7 & UE-25 UZN \#7 & .65 & $6 / 9$ & & 43 & USW UZ-N43 & .57 & $6 / 8$ & & 80 & USW UZ-N88 & .73 & $6 / 9$ & \\
\hline 8 & UE-25 UZN \#8 & .57 & $6 / 9$ & & 43 & N43 4" Gage & NI & & & 81 & USW UZ-N89 & .73 & $6 / 9$ & \\
\hline 9 & UE-25 UZN \#9 & .62 & $6 / 9$ & & 44 & USW UZ-N44 & .64 & $6 / 8$ & & 82 & USW UZ-N90 & .73 & $6 / 9$ & \\
\hline 10 & UE-25 UZN \#10 & .63 & $6 / 9$ & & 45 & USW UZ-N45 & .70 & $6 / 8$ & & 82 & N90 4" Gage & .78 & $6 / 9$ & \\
\hline 10 & N10 4" Gage & & & Removed & 46 & USW UZ-N46 & .88 & $6 / 9$ & & 83 & USW UZ-N93 & .67 & $6 / 14$ & \\
\hline 11 & UE-25 UZN \#12 & .55 & $6 / 9$ & & 47 & USW UZ-N47 & .78 & $6 / 9$ & & 84 & USW UZ-N94 & .70 & $6 / 14$ & \\
\hline 12 & UE-25 UZN \#13 & .60 & $6 / 9$ & & 48 & USW UZ-N48 & .55 & $6 / 17$ & & 85 & USW UZ-N95 & .72 & $6 / 14$ & \\
\hline 13 & UE-25 UZN \#14 & .52 & $6 / 9$ & & 49 & USW UZ-N49 & .55 & $6 / 17$ & & 86 & USW UZ-N96 & .58 & $6 / 14$ & \\
\hline 14 & UE-25 UZN \#18 & .52 & $6 / 9$ & & 50 & USW UZ-N50 & .51 & $6 / 17$ & & 87 & USW UZ-N98 & .67 & $6 / 7$ & \\
\hline 15 & UE-25 UZN \#19 & .59 & $6 / 21$ & & 50 & N50 4" Gage & NI & & & 87 & N98 4" Gage & NI & & \\
\hline 16 & UE-25 UZN \#20 & .55 & $6 / 21$ & & 51 & USW UZ-NS1 & .58 & $6 / 17$ & & 88 & USW UZ-13 & .62 & $6 / 14$ & \\
\hline 17 & UE-25 UZN \#21 & .51 & $6 / 21$ & & 52 & USW UZ-N52 & .55 & $6 / 17$ & & 88 & UZ-13 4" Gage & .66 & $6 / 14$ & \\
\hline 17 & N21 4" Gage & NI & & & 53 & USW UZ-N53 & .54 & $6 / 9$ & & 89 & USW GA-1 & .80 & $6 / 9$ & \\
\hline 18 & UE-25 UZN \#22 & .54 & $6 / 21$ & & 54 & USW UZ-N54 & .53 & $6 / 9$ & & 90 & USW G-2 & .79 & $6 / 9$ & \\
\hline 19 & UE-25 UZN \#23 & .51 & $6 / 21$ & & 54 & N54 4" Gage & .62 & $6 / 9$ & & 91 & UE25 WT-4 & $\mathbf{M}$ & & \\
\hline 20 & UE-25 UZN \#28 & .55 & $6 / 7$ & & 55 & USW UZ-N55 & .52 & $6 / 9$ & & 92 & UE25 WT-18 & $\mathbf{M}$ & & \\
\hline 21 & UE-25 UZN \#29 & .53 & $6 / 7$ & & 56 & USW UZ-N57 & .67 & $6 / 14$ & & 93 & USW H-5 & .74 & $6 / 24$ & \\
\hline 22 & UE-25 UZN \#30 & .63 & $6 / 7$ & & 57 & USW UZ-N64 & .79 & $6 / 24$ & & 94 & HRF Wedge & .88 & $6 / 7$ & \\
\hline
\end{tabular}


8 Table 2. Summary of precipitation data from the nonrecording gage network at Yucca Mountain, Nevada, for water years 1992 and 1993 --Continued

\begin{tabular}{|c|c|c|c|c|c|c|c|c|c|c|c|c|c|c|}
\hline $\begin{array}{l}\text { Loca- } \\
\text { tlon } \\
\text { code }\end{array}$ & $\begin{array}{c}\text { Slte } \\
\text { nomenclature }\end{array}$ & $\begin{array}{l}\text { Amount } \\
\text { (inches) }\end{array}$ & $\begin{array}{l}\text { Date } \\
\text { read }\end{array}$ & Notes & $\begin{array}{l}\text { Loca- } \\
\text { tion } \\
\text { code }\end{array}$ & $\begin{array}{c}\text { Site } \\
\text { nomenclature }\end{array}$ & $\begin{array}{l}\text { Amount } \\
\text { (inches) }\end{array}$ & $\begin{array}{l}\text { Date } \\
\text { read }\end{array}$ & Notes & $\begin{array}{l}\text { Loca- } \\
\text { tion } \\
\text { code }\end{array}$ & $\begin{array}{c}\text { Site } \\
\text { nomenclature }\end{array}$ & $\begin{array}{l}\text { Amount } \\
\text { (Inches) }\end{array}$ & $\begin{array}{l}\text { Date } \\
\text { read }\end{array}$ & Notes \\
\hline \multicolumn{15}{|c|}{ STORM OF JUNE 5-6, 1993-Continued } \\
\hline 23 & UE-25 UZN \#56 & .58 & $6 / 9$ & & 58 & USW UZ-N65 & $\mathrm{M}$ & $6 / 14$ & & 94 & HRF 4" Gage & .95 & $6 / 7$ & \\
\hline 24 & UE-25 UZN \#60 & .63 & $6 / 9$ & & 59 & USW UZ-N66 & .59 & $6 / 14$ & & 94 & HRF 8" Gage & .96 & $6 / 7$ & \\
\hline 25 & UE-25 UZN \#85 & .54 & $4 / 1$ & & 60 & USW UZ-N67 & .50 & $6 / 14$ & & 95 & USW H-3 & .62 & $6 / 16$ & \\
\hline 26 & UE-25 UZN \#92 & .43 & $6 / 7$ & & 60 & N67 4" Gage & .57 & $6 / 14$ & & 96 & Wea station & .78 & $6 / 24$ & \\
\hline 26 & N92 4" Gage & .49 & $6 / 7$ & & 61 & USW UZ-N68 & $\mathbf{M}$ & $6 / 14$ & & 97 & Wea station & M & & \\
\hline 27 & UE-25 UZN \#97 & .56 & $6 / 7$ & & 62 & USW UZ-N69 & .56 & $6 / 14$ & & 98 & Evaporation & .81 & $6 / 7$ & \\
\hline 28 & UE-29 UZN \#91 & .63 & $6 / 7$ & & 63 & USW UZ-N70 & .69 & $6 / 9$ & & 99 & Wea station & .66 & $6 / 14$ & \\
\hline 28 & N91 4" Gage & .73 & $6 / 7$ & & 63 & N70 4" Gage & .76 & $6 / 9$ & & 99 & Wea station & .66 & $6 / 14$ & \\
\hline 29 & USW UZ-N11 & .72 & $6 / 9$ & & 64 & USW UZ-N71 & .70 & $6 / 24$ & & 100 & G-3 & .49 & $6 / 14$ & \\
\hline 29 & N11 4" Gage & .71 & $6 / 9$ & & 64 & N71 4" Gage & .74 & $6 / 24$ & & 100 & G-3 8" Gage & .54 & $6 / 14$ & \\
\hline 30 & USW UZ-N15 & .80 & $6 / 9$ & & 65 & USW UZ-N72 & .80 & $6 / 24$ & & 101 & Wea station & .57 & $6 / 9$ & \\
\hline 31 & USW UZ-N16 & .74 & $6 / 9$ & & 66 & USW UZ-N73 & .76 & $6 / 24$ & & 101 & Wea station & .60 & $6 / 9$ & \\
\hline 32 & USW UZ-N17 & .79 & $6 / 9$ & & 67 & USW UZ-N74 & .67 & $6 / 24$ & & 102 & Wea station & .88 & $6 / 7$ & \\
\hline 33 & USW UZ-N24 & .71 & $6 / 9$ & & 68 & USW UZ-N75 & .70 & $6 / 24$ & & 103 & Wea station & .77 & $6 / 9$ & \\
\hline 33 & N24 4" Gage & NI & & & 68 & N75 4" Gage & .77 & $6 / 24$ & & 103 & Wea station & .80 & $6 / 9$ & \\
\hline 34 & USW UZ-N25 & .72 & $6 / 9$ & & 69 & USW UZ-N76 & .72 & $6 / 24$ & & 104 & Fran Ridge & .48 & $6 / 14$ & \\
\hline 35 & USW UZ-N26 & .74 & $6 / 9$ & & 70 & USW UZ-N77 & .69 & $6 / 9$ & & 104 & Fran Ridge (8") & .56 & $6 / 14$ & \\
\hline 36 & USW UZ-N27 & .74 & $6 / 16$ & & 71 & USW UZ-N78 & .61 & $6 / 9$ & & 105 & Plug Hill & .61 & $6 / 9$ & \\
\hline 36 & N27 8" Gage & .80 & $6 / 16$ & & 72 & USW UZ-N79 & .67 & $6 / 9$ & & 106 & 4JA (8") & .92 & $6 / 7$ & \\
\hline \multirow[t]{2}{*}{37} & USW UZ-N35 & .63 & $6 / 9$ & & 73 & USW UZ-N80 & .65 & $6 / 9$ & & 107 & Knothead Gap & .56 & $6 / 9$ & \\
\hline & & & & & & & & & & 108 & Upper Forty Mile & .50 & $6 / 7$ & \\
\hline
\end{tabular}


Table 2. Summary of precipitation data from the nonrecording gage network at Yucca Mountain, Nevada, for water years 1992 and 1993 --Continued

\begin{tabular}{|c|c|c|c|c|c|c|c|c|c|c|c|c|c|c|}
\hline $\begin{array}{l}\text { Loca- } \\
\text { tion } \\
\text { code }\end{array}$ & $\begin{array}{c}\text { Site } \\
\text { nomenclature }\end{array}$ & $\begin{array}{l}\text { Amount } \\
\text { (inches) }\end{array}$ & $\begin{array}{l}\text { Date } \\
\text { read }\end{array}$ & Notes & $\begin{array}{l}\text { Loca- } \\
\text { tion } \\
\text { code }\end{array}$ & $\begin{array}{c}\text { Silte } \\
\text { nomenclature }\end{array}$ & $\begin{array}{l}\text { Amount } \\
\text { (Inches) }\end{array}$ & $\begin{array}{l}\text { Date } \\
\text { read }\end{array}$ & Notes & $\begin{array}{l}\text { Loca- } \\
\text { tlon } \\
\text { code }\end{array}$ & $\begin{array}{c}\text { Site } \\
\text { nomenclature }\end{array}$ & $\begin{array}{l}\text { Amount } \\
\text { (inches) }\end{array}$ & $\begin{array}{l}\text { Date } \\
\text { read }\end{array}$ & Notes \\
\hline \multicolumn{15}{|c|}{ STORM OF AUGUST 4, 1993} \\
\hline 1 & UE-25 UZN \#1 & .14 & $8 / 9$ & & 38 & USW UZ-N36 & .16 & $8 / 9$ & & 73 & N80 4" Gage & .15 & $8 / 10$ & \\
\hline 2 & UE-25 UZN \#2 & .17 & $8 / 9$ & & 39 & USW UZ-N37 & .11 & $8 / 12$ & & 74 & USW UZ-N81 & .12 & $8 / 10$ & \\
\hline 3 & UE-25 UZN \#3 & .11 & $8 / 9$ & & 39 & N37 4" Gage & .15 & $8 / 12$ & & 75 & USW UZ-N82 & .11 & $8 / 10$ & \\
\hline 4 & UE-25 UZN \#4 & .16 & $8 / 9$ & & 40 & USW UZ-N40 & .08 & $8 / 12$ & & 76 & USW UZ-N83 & .11 & $8 / 10$ & \\
\hline 4 & N4 4" Gage & & & Removed & 40 & N40 4" Gage & NI & & & 77 & USW UZ-N84 & .14 & $8 / 10$ & \\
\hline 5 & UE-25 UZN \#5 & .10 & $8 / 9$ & & 41 & USW UZ-N41 & .07 & $8 / 12$ & & 78 & USW UZ-N86 & .09 & $8 / 10$ & \\
\hline 6 & UE-25 UZN \#6 & .12 & $8 / 9$ & & 42 & USW UZ-N42 & .06 & $8 / 12$ & & 79 & USW UZ-N87 & .12 & $8 / 10$ & \\
\hline 7 & UE-25 UZN \#7 & .15 & $8 / 9$ & & 43 & USW UZ-N43 & .07 & $8 / 12$ & & 80 & USW UZ-N88 & .09 & $8 / 10$ & \\
\hline 8 & UE-25 UZN \#8 & .10 & $8 / 9$ & & 43 & N43 4" Gage & NI & & & 81 & USW UZ-N89 & .12 & $8 / 10$ & \\
\hline 9 & UE-25 UZN \#9 & .15 & 8/9 & & 44 & USW UZ-N44 & .11 & $8 / 12$ & & 82 & USW UZ-N90 & .12 & $8 / 10$ & \\
\hline 10 & UE-25 UZN \#10 & .16 & 8/9 & & 45 & USW UZ-N45 & .12 & $8 / 12$ & & 82 & N90 4", Gage & .15 & $8 / 10$ & \\
\hline 10 & N10 4" Gage & & & Removed & 46 & USW UZ-N46 & .16 & $8 / 9$ & & 83 & USW UZ-N93 & .12 & $8 / 18$ & \\
\hline 11 & UE-25 UZN \#12 & .08 & $8 / 9$ & & 47 & USW UZ-N47 & .14 & $8 / 9$ & & 84 & USW UZ-N94 & .12 & $8 / 18$ & \\
\hline 12 & UE-25 UZN \#13 & .12 & $8 / 9$ & & 48 & USW UZ-N48 & .07 & $8 / 19$ & & 85 & USW UZ-N95 & .12 & $8 / 18$ & \\
\hline 13 & UE-25 UZN \#14 & .08 & $8 / 9$ & & 49 & USW UZ-N49 & .08 & $8 / 19$ & & 86 & USW UZ-N96 & .12 & $8 / 18$ & \\
\hline 14 & UE-25 UZN \#18 & .11 & $8 / 12$ & & 50 & USW UZ-N50 & .09 & $8 / 19$ & & 87 & USW UZ-N98 & .15 & $8 / 12$ & \\
\hline 15 & UE-25 UZN \#19 & .08 & $8 / 19$ & & 50 & N50 4" Gage & NI & & & 87 & N98 4" Gage & NI & & \\
\hline 16 & UE-25 UZN \#20 & .05 & $8 / 19$ & & 51 & USW UZ-N51 & .08 & $8 / 19$ & & 88 & USW UZ 13 & .05 & $8 / 26$ & \\
\hline 17 & UE-25 UZN \#21 & .05 & $8 / 19$ & & 52 & USW UZ-N52 & .07 & $8 / 19$ & & 88 & UZ-13 4" Gag & .09 & $8 / 26$ & \\
\hline 17 & N21 4" Gage & NI & & & 53 & USW UZ-N53 & .09 & $8 / 19$ & & 89 & USW GA-1 & .20 & $8 / 9$ & \\
\hline 18 & UE-25 UZN \#22 & .08 & $8 / 19$ & & 54 & USW UZ-N54 & .09 & $8 / 11$ & & 90 & USW G-2 & .17 & $8 / 9$ & \\
\hline 19 & UE-25 UZN \#23 & .07 & $8 / 19$ & & 54 & N54 4" Gage & .10 & $8 / 10$ & & 91 & UE25 WT-4 & .11 & $8 / 9$ & \\
\hline 20 & UE-25 UZN \#28 & .06 & $8 / 19$ & & 55 & USW UZ-NSS & .10 & $8 / 19$ & & 92 & UE25 WT-18 & .20 & $8 / 9$ & \\
\hline 21 & UE-25 UZN \#29 & .06 & $8 / 19$ & & 56 & USW UZ-N57 & 0 & $8 / 27$ & & 93 & USW H-5 & .14 & $8 / 27$ & \\
\hline 22 & UE-25 UZN \#30 & .09 & $8 / 19$ & & 57 & USW UZ-N64 & $\mathbf{0}$ & $8 / 27$ & & 94 & HRF Wedge & .02 & $8 / 5$ & \\
\hline
\end{tabular}




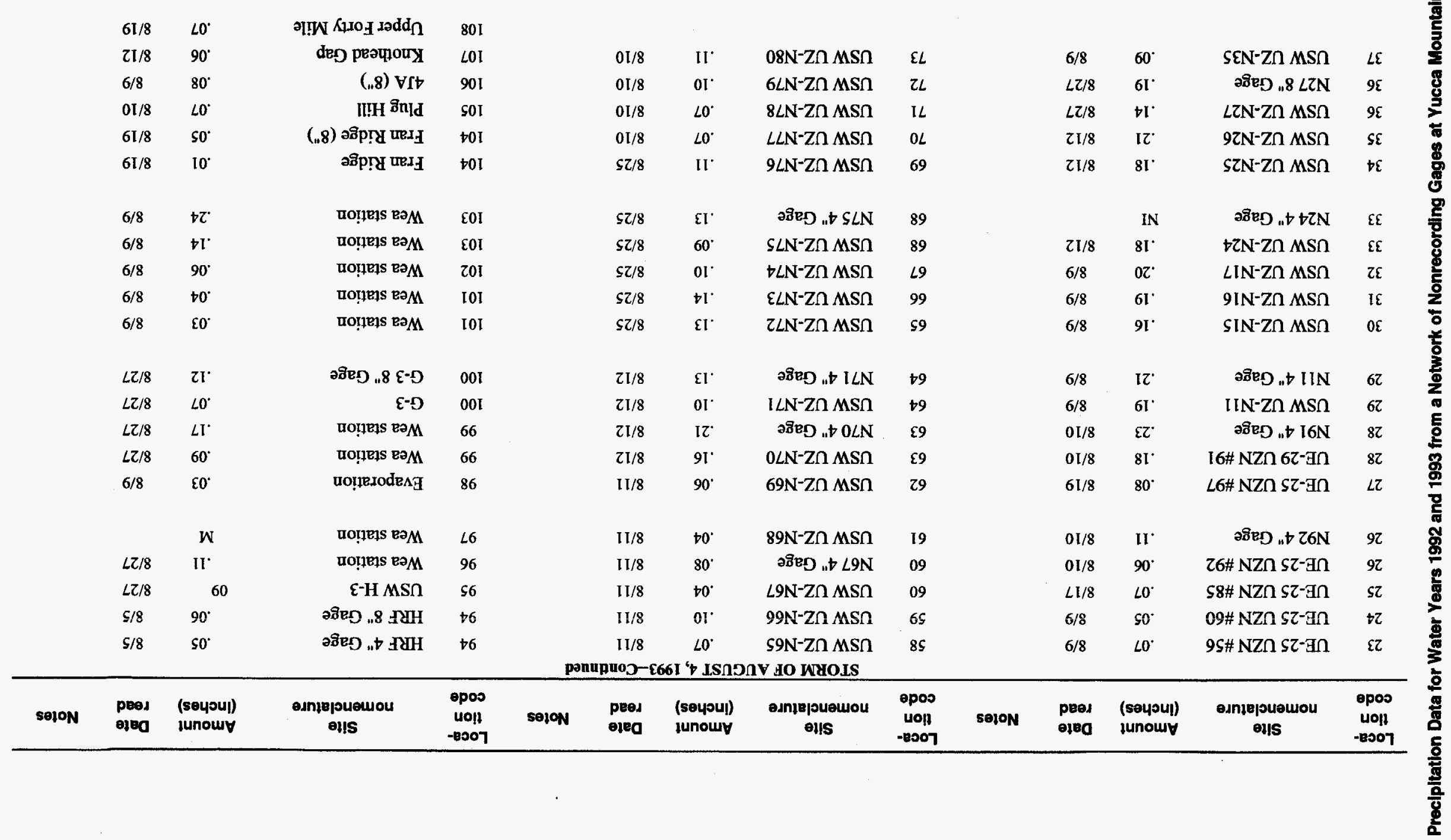


Table 2. Summary of precipitation data from the nonrecording gage network at Yucca Mountain, Nevada, for water years 1992 and 1993 --Continued

\begin{tabular}{|c|c|c|c|c|c|c|c|c|c|c|c|c|c|c|}
\hline $\begin{array}{l}\text { Loca- } \\
\text { tion } \\
\text { code }\end{array}$ & $\begin{array}{c}\text { Site } \\
\text { nomenclature }\end{array}$ & $\begin{array}{l}\text { Amount } \\
\text { (Inches) }\end{array}$ & $\begin{array}{l}\text { Date } \\
\text { read }\end{array}$ & Notes & $\begin{array}{l}\text { Loca- } \\
\text { tion } \\
\text { code }\end{array}$ & $\begin{array}{c}\text { Site } \\
\text { nomenclature }\end{array}$ & $\begin{array}{l}\text { Amount } \\
\text { (inches) }\end{array}$ & $\begin{array}{l}\text { Date } \\
\text { read }\end{array}$ & Notes & $\begin{array}{l}\text { Loca- } \\
\text { tion } \\
\text { code }\end{array}$ & $\begin{array}{c}\text { Site } \\
\text { nomenclature }\end{array}$ & $\begin{array}{l}\text { Amount } \\
\text { (inches) }\end{array}$ & $\begin{array}{l}\text { Date } \\
\text { read }\end{array}$ & Notes \\
\hline \multicolumn{15}{|c|}{ STORM OF AUGUST 27, 1993} \\
\hline 1 & UE-25 UZN \#1 & .02 & $8 / 30$ & & 38 & USW UZ-N36 & .08 & $8 / 30$ & & 73 & N80 4" Gage & .35 & $9 / 1$ & \\
\hline 2 & UE-25 UZN \#2 & .02 & $8 / 30$ & & 39 & USW UZ-N37 & 0 & $8 / 30$ & & 74 & USW UZ-N81 & .14 & $9 / 1$ & \\
\hline 3 & UE-25 UZN \#3 & .01 & $8 / 30$ & & 39 & N37 4" Gage & $\mathrm{T}$ & $8 / 30$ & & 75 & USW UZ-N82 & .14 & $9 / 1$ & \\
\hline 4 & UE-25 UZN \#4 & .01 & $8 / 30$ & & 40 & USW UZ-N40 & 0 & $8 / 30$ & & 76 & USW UZ-N83 & .07 & $9 / 1$ & \\
\hline 4 & N4 4" Gage & & & Removed & 40 & N40 4" Gage & $\mathrm{T}$ & $8 / 30$ & & 77 & USW UZ-N84 & .04 & $9 / 1$ & \\
\hline 5 & UE-25 UZN \#5 & 0 & $8 / 30$ & & 41 & USW UZ-N4I & $\mathrm{T}$ & $8 / 30$ & @ & 78 & USW UZ-N86 & .08 & 9/1 & \\
\hline 6 & UE-25 UZN \#6 & .01 & $8 / 30$ & & 42 & USW UZ-N42 & $\mathrm{T}$ & $8 / 30$ & $@$ & 79 & USW UZ-N87 & .05 & $9 / 1$ & \\
\hline 7 & UE-25 UZN \#7 & .02 & $8 / 30$ & & 43 & USW UZ-N43 & 0 & $8 / 30$ & & 80 & USW UZ-N88 & .03 & $9 / 1$ & \\
\hline 8 & UE-25 UZN \#8 & 0 & $8 / 30$ & & 43 & N43 4" Gage & $\mathbf{T}$ & $8 / 30$ & & 81 & USW UZ-N89 & .04 & $9 / 1$ & \\
\hline 9 & UE-25 UZN \#9 & .01 & $8 / 30$ & & 44 & USW UZ-N44 & .01 & $8 / 30$ & & 82 & USW UZ-N90 & .05 & $9 / 1$ & \\
\hline 10 & UE-25 UZN \#10 & .02 & $8 / 30$ & & 45 & USW UZ-N45 & .01 & $8 / 30$ & & 82 & N90 4" Gage & .07 & 9/1 & \\
\hline 10 & N10 4" Gage & & & Removed & 46 & USW UZ-N46 & .05 & $8 / 30$ & & 83 & USW UZ-N93 & .20 & $8 / 27$ & \\
\hline 11 & UE-25 UZN \#12 & 0 & $8 / 30$ & & 47 & USW UZ-N47 & .05 & $8 / 30$ & & 84 & USW UZ-N94 & .20 & $9 / 3$ & \\
\hline 12 & UE-25 UZN \#13 & 0 & $8 / 30$ & & 48 & USW UZ-N48 & .19 & $8 / 30$ & @ & 85 & USW UZ-N95 & .21 & $9 / 3$ & \\
\hline 13 & UE-25 UZN \#14 & 0 & $8 / 30$ & & 49 & USW UZ-N49 & .24 & $8 / 30$ & @ & 86 & USW UZ-N96 & .19 & $9 / 3$ & \\
\hline 14 & UE-25 UZN \#18 & 0 & $8 / 30$ & & 50 & USW UZ-NSO & .15 & $8 / 30$ & & 87 & USW UZ-N98 & 0 & $8 / 30$ & \\
\hline 15 & UE-25 UZN \#19 & .02 & $8 / 30$ & & 50 & N50 4" Gage & .15 & $8 / 30$ & & 87 & N98 4" Gage & $\mathrm{T}$ & $8 / 30$ & \\
\hline 16 & UE-25 UZN \#20 & .01 & $8 / 30$ & @ & 51 & USW UZ-N51 & .15 & $8 / 30$ & & 88 & USW UZ-13 & .39 & $8 / 27$ & \\
\hline 17 & UE-25 UZN \#21 & .01 & $8 / 30$ & & 52 & USW UZ-N52 & .15 & $8 / 30$ & & 88 & UZ-13 4" Gage & .44 & $8 / 27$ & \\
\hline 17 & N21 4" Gage & .02 & $8 / 30$ & & 53 & USW UZ-NS3 & .03 & $8 / 30$ & & 89 & USW GA-1 & .04 & $8 / 30$ & \\
\hline 18 & UE-25 UZN \#22 & .01 & $8 / 30$ & & 54 & USW UZ-N54 & .06 & $8 / 30$ & & 90 & USW G-2 & .05 & $8 / 30$ & \\
\hline 19 & UE-25 UZN \#23 & .02 & $8 / 30$ & @ & 54 & N54 4" Gage & .06 & $8 / 30$ & & 91 & UE25 WT-4 & 0 & $8 / 30$ & \\
\hline 20 & UE-25 UZN \#28 & .01 & $8 / 30$ & & 55 & USW UZ-N55 & .07 & $8 / 30$ & & 92 & UE25 WT-18 & .02 & $8 / 30$ & \\
\hline 21 & UE-25 UZN \#29 & .03 & $8 / 30$ & @ & 56 & USW UZ-N57 & .32 & $8 / 27$ & & 93 & USW H-5 & $\mathrm{T}$ & $8 / 27$ & \\
\hline 22 & UE-25 UZN \#30 & .04 & $8 / 30$ & & 57 & USW UZ-N64 & 0 & $9 / 3$ & & 94 & HRF Wedge & 0 & $8 / 27$ & \\
\hline
\end{tabular}


4 Table 2. Summary of precipitation data from the nonrecording gage network at Yucca Mountain, Nevada, for water years 1992 and 1993 --Continued

\begin{tabular}{|c|c|c|c|c|c|c|c|c|c|c|c|c|c|c|}
\hline $\begin{array}{l}\text { Loce- } \\
\text { tion } \\
\text { code }\end{array}$ & $\begin{array}{c}\text { Site } \\
\text { nomenclature }\end{array}$ & $\begin{array}{l}\text { Amount } \\
\text { (Inches) }\end{array}$ & $\begin{array}{l}\text { Date } \\
\text { read }\end{array}$ & Notes & $\begin{array}{l}\text { Loca- } \\
\text { tion } \\
\text { code }\end{array}$ & $\begin{array}{c}\text { Site } \\
\text { nomenclature }\end{array}$ & $\begin{array}{l}\text { Amount } \\
\text { (inches) }\end{array}$ & $\begin{array}{l}\text { Date } \\
\text { read }\end{array}$ & Notes & $\begin{array}{l}\text { Loce- } \\
\text { tion } \\
\text { code }\end{array}$ & $\begin{array}{c}\text { Site } \\
\text { nomenclature }\end{array}$ & $\begin{array}{l}\text { Amount } \\
\text { (Inches) }\end{array}$ & $\begin{array}{l}\text { Date } \\
\text { read }\end{array}$ & Notes \\
\hline \multicolumn{15}{|c|}{ STORM OF AUGUST 27, 1993-Contlmued } \\
\hline 23 & UE-25 UZN \#56 & .05 & $8 / 30$ & $@$ & 58 & USW UZ-N65 & .21 & $9 / 3$ & & 94 & HRF 4" Gage & 0 & $8 / 27$ & \\
\hline 24 & UE-25 UZN \#60 & .04 & $8 / 30$ & & $5 \hat{y}$ & USW UZZ-N66 & .19 & $9 / 3$ & & 94 & HRF 8" Gage & 0 & $8 / 27$ & \\
\hline 25 & UE-25 UZN \#85 & .06 & $8 / 27$ & & 60 & USW UZ-N67 & .11 & $8 / 27$ & & 95 & USW H-3 & .62 & $9 / 3$ & \\
\hline 26 & UE-25 UZN \#92 & .05 & $8 / 30$ & & 60 & N67 4" Gage & .19 & $8 / 27$ & & 96 & Wea station & .20 & $9 / 3$ & \\
\hline 26 & N92 4" Gage & .09 & $8 / 30$ & & 61 & USW UZ-N68 & .17 & $8 / 27$ & $@$ & 97 & Wea station & M & & \\
\hline 27 & UE-25 UZN \#97 & .03 & $8 / 30$ & & 62 & USW UZ-N69 & .17 & $8 / 27$ & & 98 & Evaporation & 0 & $8 / 27$ & \\
\hline 28 & UE-29 UZN \#91 & .03 & $8 / 30$ & & 63 & USW UZ-N70 & .05 & $8 / 30$ & & 99 & Wea station & .47 & $8 / 27$ & \\
\hline 28 & N91 4" Gage & .04 & $8 / 30$ & & 63 & N70 4" Gage & .06 & $8 / 30$ & & 99 & Wea station & .49 & $8 / 27$ & \\
\hline 29 & USW UZ-NII & 0 & $8 / 30$ & & 64 & USW UZ-N71 & .06 & $9 / 3$ & & 100 & G-3 & .29 & $8 / 27$ & \\
\hline 29 & N11 4" Gage & 0 & $8 / 30$ & & 64 & N71 4" Gage & .08 & $9 / 3$ & & 100 & G-3 8" Gage & .35 & $8 / 27$ & \\
\hline 30 & USW UZ-N15 & .10 & $8 / 30$ & & 65 & USW UZ-N72 & .06 & $9 / 3$ & & 101 & Wea station & .03 & $8 / 30$ & \\
\hline 31 & USW UZ-N16 & .09 & $8 / 30$ & & 66 & USW UZ-N73 & .08 & $9 / 3$ & & 101 & Wea station & .03 & $8 / 30$ & \\
\hline 32 & USW UZ-N17 & .11 & $8 / 30$ & & 67 & USW UZ-N74 & .07 & $9 / 3$ & & 102 & Wea station & 0 & $9 / 3$ & \\
\hline 33 & USW UZ-N24 & 0 & $8 / 30$ & & 68 & USW UZ-N75 & .08 & $9 / 3$ & & 103 & Wea station & .04 & $8 / 30$ & \\
\hline 33 & N24 4" Gage & $\mathrm{T}$ & $8 / 30$ & & 68 & N75 4" Gage & .08 & $9 / 3$ & & 103 & Wea station & .08 & $8 / 30$ & \\
\hline 34 & USW UZ-N25 & .01 & $8 / 30$ & @ & 69 & USW UZ-N76 & .08 & 9/3 & & 104 & Fran Ridge & 0 & $8 / 30$ & \\
\hline 35 & USW UZ-N26 & .02 & $8 / 30$ & $@$ & 70 & USW UZ-N77 & .65 & $9 / 1$ & & 104 & Fran Ridge (8") & 0 & $8 / 30$ & \\
\hline 36 & USW UZ-N27 & 0 & $9 / 3$ & & 71 & USW UZ-N78 & .29 & 9/1 & & 105 & Plug Hill & .30 & $9 / 1$ & \\
\hline 36 & N27 8" Gage & & 0 & $9 / 3$ & 72 & USW UZ-N79 & .25 & $9 / 1$ & & 106 & 4JA (8") & 0 & $9 / 3$ & \\
\hline \multirow[t]{2}{*}{37} & USW UZ-N35 & .10 & $8 / 30$ & & 73 & USW UZ-N80 & .25 & $9 / 1$ & & 107 & Knothead Gap & .01 & $8 / 30$ & \\
\hline & & & & & & & & & & 108 & Upper Forty Mile & .11 & $8 / 30$ & \\
\hline
\end{tabular}


Table 2. Summary of precipitation data from the nonrecording gage network at Yucca Mountain, Nevada, for water years 1992 and $1993--$ Continued

\begin{tabular}{|c|c|c|c|c|c|c|c|c|c|c|c|c|c|c|}
\hline $\begin{array}{l}\text { Loca- } \\
\text { tion } \\
\text { code }\end{array}$ & $\begin{array}{c}\text { Site } \\
\text { nomenclature }\end{array}$ & $\begin{array}{l}\text { Amount } \\
\text { (inches) }\end{array}$ & $\begin{array}{l}\text { Date } \\
\text { read }\end{array}$ & Notes & $\begin{array}{l}\text { Loca- } \\
\text { tlon } \\
\text { code }\end{array}$ & $\begin{array}{c}\text { Sile } \\
\text { nomenelature }\end{array}$ & $\begin{array}{l}\text { Amount } \\
\text { (inches) }\end{array}$ & $\begin{array}{l}\text { Date } \\
\text { read }\end{array}$ & Notes & $\begin{array}{l}\text { Loca- } \\
\text { tion } \\
\text { code }\end{array}$ & $\begin{array}{c}\text { Site } \\
\text { nomenclature }\end{array}$ & $\begin{array}{l}\text { Amount } \\
\text { (inches) }\end{array}$ & $\begin{array}{l}\text { Date } \\
\text { read }\end{array}$ & Notes \\
\hline \multicolumn{15}{|c|}{ STORM OF SEPTEMBER 17,1993} \\
\hline 1 & UE-25 UZN \#1 & .10 & 9/21 & & 38 & USW UZ-N36 & .25 & $10 / 4$ & & 73 & N80 4" Gage & $\mathbf{0}$ & $10 / 7$ & \\
\hline 2 & UE-25 UZN \#2 & .08 & 9/21 & & 39 & USW UZ-N37 & .05 & $10 / 5$ & & 74 & USW UZ-N81 & 0 & $10 / 7$ & \\
\hline 3 & UE-25 UZN \#3 & .06 & $9 / 21$ & & 39 & N37 4" Gage & .07 & $10 / 5$ & & 75 & USW UZ-N82 & 0 & $10 / 7$ & \\
\hline 4 & UE-25 UZN \#4 & .10 & 9/21 & & 40 & USW UZ-N40 & .05 & $9 / 21$ & & 76 & USW UZ-N83 & 0 & $10 / 7$ & \\
\hline 4 & N4 4" Gage. & & & Removed & 40 & N40 4" Gage & .06 & $9 / 21$ & & 77 & USW UZ-N84 & $\mathbf{0}$ & $10 / 7$ & \\
\hline 5 & UE-25 UZN \#5 & .08 & 9/21 & & 41 & USW UZ-N4I & .06 & 9/21 & & 78 & USW UZ-N86 & 0 & 9/1 & \\
\hline 6 & UE-25 UZN \#6 & .07 & 9/21 & & 42 & USW UZ-N42 & .07 & $9 / 21$ & & 79 & USW UZ-N87 & 0 & 9/1 & \\
\hline 7 & UE-25 UZN \#7 & .09 & 9/21 & & 43 & USW UZ-N43 & .04 & $9 / 21$ & & 80 & USW UZ-N88 & $\mathbf{0}$ & $9 / 1$ & \\
\hline 8 & UE-25 UZN \#8 & .06 & 9/21 & & 43 & N43 4" Gage & .06 & $9 / 21$ & & 81 & USW UZ-N89 & 0 & $9 / 1$ & \\
\hline 9 & UE-25 UZN \#9 & .10 & $9 / 21$ & & 44 & USW UZ-N44 & .05 & $9 / 21$ & & 82 & USW UZ-N90 & 0 & $9 / 1$ & \\
\hline 10 & UE-25 UZN \#10 & .11 & 9/21 & & 45 & USW UZ-N45 & .05 & $9 / 21$ & & 82 & N90 4" Gage & 0 & $9 / 1$ & \\
\hline 10 & N10 4" Gage & & & Removed & 46 & USW UZ-N46 & .15 & 9/20 & & 83 & USW UZ-N93 & .01 & $9 / 23$ & \\
\hline 11 & UE-25 UZN \#12 & .06 & 9/21 & & 47 & USW UZ-N47 & .13 & $9 / 20$ & & 84 & USW UZ-N94 & 0 & 9/23 & \\
\hline 12 & UE-25 UZN \#13 & .08 & 9/21 & & 48 & USW UZ-N48 & .01 & $9 / 21$ & & 85 & USW UZ-N95 & 0 & 9/23 & \\
\hline 13 & UE-25 UZN \#14 & .05 & 9/21 & & 49 & USW UZ-N49 & .01 & $9 / 21$ & & 86 & USW UZ-N96 & 0 & 9/23 & \\
\hline 14 & UE-25 UZN \#18 & .05 & $9 / 21$ & & 50 & USW UZ-N50 & 0 & 9/21 & & 87 & USW UZ-N98 & .05 & $9 / 20$ & \\
\hline 15 & UE-25 UZN \#19 & .06 & $9 / 21$ & & 50 & N50 4" Gage & .02 & $9 / 21$ & & 87 & N98 4" Gage & .14 & $9 / 20$ & \\
\hline 16 & UE-25 UZN \#20 & .05 & 9/21 & & 51 & USW UZ-NS1 & .03 & $9 / 21$ & & 88 & USW UZ-13 & 0 & 9/23 & \\
\hline 17 & UE-25 UZN \#21 & .05 & 9/21 & & 52 & USW UZ-NS2 & .02 & $9 / 21$ & & 88 & UZ-13 4" Gage & 0 & 9/23 & \\
\hline 17 & N21 4" Gage & .05 & $9 / 21$ & & 53 & USW UZ-N53 & .01 & $9 / 21$ & & 89 & USW GA-1 & .29 & $9 / 20$ & \\
\hline 18 & UE-25 UZN \#22 & .08 & $9 / 21$ & & 54 & USW UZ-N54 & 0 & $9 / 21$ & & 90 & USW G-2 & .44 & $9 / 20$ & \\
\hline 19 & UE-25 UZN \#23 & .06 & $9 / 21$ & & 54 & N54 4" Gage & .03 & $9 / 21$ & & 91 & UE25 WT-4 & .07 & 9/20 & \\
\hline 20 & UE-25 UZN \#28 & .04 & $9 / 21$ & & 55 & USW UZ-N55 & .02 & 9/21 & & 92 & UE25 WT-18 & .17 & $9 / 20$ & \\
\hline 21 & UE-25 UZN \#29 & .04 & 9/21 & & 56 & USW UZ-N57 & $\mathbf{T}$ & $10 / 8$ & & 93 & USW H-5 & 0 & $8 / 27$ & \\
\hline 22 & UE-25 UZN \#30 & .04 & 9/21 & & 57 & USW UZ-N64 & .06 & $9 / 23$ & & 94 & HRF Wedge & 0 & $9 / 20$ & \\
\hline
\end{tabular}


\& Table 2. Summary of precipitation data from the nonrecording gage network at Yucca Mountain, Nevada, for water years 1992 and $1993--$ Continued

\begin{tabular}{|c|c|c|c|c|c|c|c|c|c|c|c|c|c|}
\hline $\begin{array}{l}\text { Loca- } \\
\text { tlon } \\
\text { code }\end{array}$ & $\begin{array}{c}\text { Site } \\
\text { nomenclature }\end{array}$ & $\begin{array}{l}\text { Amount } \\
\text { (inches) }\end{array}$ & $\begin{array}{l}\text { Date } \\
\text { read }\end{array}$ & Notes & $\begin{array}{l}\text { Loca- } \\
\text { tion } \\
\text { code }\end{array}$ & $\begin{array}{c}\text { Site } \\
\text { nomenclature }\end{array}$ & $\begin{array}{l}\text { Amount } \\
\text { (inches) }\end{array}$ & $\begin{array}{l}\text { Date } \\
\text { read }\end{array}$ & Notes & $\begin{array}{l}\text { Loca- } \\
\text { tion } \\
\text { code }\end{array}$ & $\begin{array}{c}\text { Site } \\
\text { nomenclature }\end{array}$ & $\begin{array}{l}\text { Amount } \\
\text { (Inches) }\end{array}$ & $\begin{array}{l}\text { Date } \\
\text { read }\end{array}$ \\
\hline \multicolumn{14}{|c|}{ STORM OF SEPTEMBER 17, 1993-Continued } \\
\hline 23 & UE-25 UZN \#56 & .01 & 9/21 & & 58 & USW UZ-N65 & .01 & $9 / 23$ & & 94 & HRF 4" Gage & 0 & $9 / 20$ \\
\hline 24 & UE-25 UZN \#60 & .02 & $9 / 21$ & & 59 & USW UZ-N66 & .01 & $9 / 23$ & & 94 & HRF 8" Gage & .01 & 9/20 \\
\hline 25 & UE-25 UZN \#85 & .01 & $10 / 8$ & & 60 & USW UZ-N67 & 0 & $10 / 8$ & & 95 & USW H-3 & 0 & 9/23 \\
\hline 26 & UE-25 UZN \#92 & M & & & 60 & N67 4" Gage & $\mathrm{T}$ & $10 / 8$ & & 96 & Wea station & 0 & $9 / 23$ \\
\hline 26 & N92 4" Gage & M & & & 61 & USW UZ-N68 & $\mathrm{T}$ & $10 / 8$ & & 97 & Wea station & $\mathbf{M}$ & \\
\hline 27 & UE-25 UZN \#97 & .05 & 9/21 & & 62 & USW UZ-N69 & $\mathrm{T}$ & $10 / 8$ & & 98 & Evaporation & 0 & $10 / 5$ \\
\hline 28 & UE-29 UZN \#91 & M & & & 63 & USW UZ-N70 & .08 & 9/20 & & 99 & Wea station & .01 & 9/23 \\
\hline 28 & N91 4" Gage & M & & & 63 & N70 4" Gage & .10 & $9 / 20$ & & 99 & Wea station & .01 & $9 / 23$ \\
\hline 29 & USW UZ-N11 & .22 & $9 / 20$ & & 64 & USW UZ-N71 & .01 & $\mathscr{B}$ & & 100 & G-3 & 0 & $9 / 23$ \\
\hline 29 & N11 4" Gage & .22 & $9 / 20$ & & 64 & N71 4" Gage & .02 & $\mathscr{B}$ & & 100 & G-3 8" Gage & 0 & $9 / 23$ \\
\hline 30 & USW UZ-N15 & .45 & $9 / 20$ & & 65 & USW UZ-N72 & 0 & $\mathscr{B}$ & & 101 & Wea station & .02 & $9 / 21$ \\
\hline 31 & USW UZ-N16 & .51 & $9 / 20$ & & 66 & USW UZ-N73 & .01 & $\mathscr{B}$ & & 101 & Wea station & .03 & 9/21 \\
\hline 32 & USW UZ-N17 & .61 & 9/20 & & 67 & USW UZ-N74 & .02 & $\mathscr{B}$ & & 102 & Wea station & .01 & $10 / 5$ \\
\hline 33 & USW UZ-N24 & .07 & 9/20 & & 68 & USW UZ-N75 & .01 & $\mathscr{B}$ & & 103 & Wea station & .07 & 9/20 \\
\hline 33 & N24 4" Gage & .10 & 9/20 & & 68 & N75 4" Gage & .02 & $\mathscr{B}$ & & 103 & Wea station & .08 & $9 / 20$ \\
\hline 34 & USW UZ-N25 & .10 & 9/20 & & 69 & USW UZ-N76 & .01 & $\mathscr{B}$ & & 104 & Fran Ridge & .02 & $9 / 21$ \\
\hline 35 & USW UZ-N26 & .11 & 9/20 & & 70 & USW UZ-N77 & 0 & $10 / 7$ & & 104 & Fran Ridge (8") & .03 & 9/21 \\
\hline 36 & USW UZ-N27 & .10 & $9 / 23$ & & 71 & USW UZ-N78 & 0 & $10 / 7$ & & 105 & Plug Hill & 0 & $9 / 1$ \\
\hline 36 & N27 8" Gage & .13 & $9 / 23$ & & 72 & USW UZ-N79 & 0 & $10 / 7$ & & 106 & 4JA (8") & .01 & $9 / 21$ \\
\hline 37 & USW UZ-N35 & .04 & 9/21 & & 73 & USW UZ-N80 & 0 & $10 / 7$ & & 107 & Knothead Gap & .04 & $9 / 21$ \\
\hline & & & & & & & & & & 108 & Upper Forty Mile & $\mathbf{M}$ & \\
\hline
\end{tabular}


Table 3. Network precipitation totals for water year 1992

[Includes only sites that have a complete annual total]

\begin{tabular}{|c|c|c|c|c|c|}
\hline $\begin{array}{l}\text { Location } \\
\text { code }\end{array}$ & $\begin{array}{c}\text { Site } \\
\text { nomenclature }\end{array}$ & $\begin{array}{c}\text { Total } \\
\text { (inches) }\end{array}$ & $\begin{array}{l}\text { Location } \\
\text { code }\end{array}$ & $\begin{array}{c}\text { Site } \\
\text { nomenclature }\end{array}$ & $\begin{array}{c}\text { Total } \\
\text { (inches) }\end{array}$ \\
\hline 1 & UE-25 UZN \#1 & 8.17 & 33 & USW UZ-N24 & 9.36 \\
\hline 3 & UE-25 UZN \#3 & 8.40 & 34 & USW UZ-N25 & 9.22 \\
\hline 4 & UE-25 UZN \#4 & 8.54 & 35 & USW UZ-N26 & 9.18 \\
\hline 5 & UE-25 UZN \#5 & 8.40 & 36 & USW UZ-N27 & 8.75 \\
\hline 6 & UE-25 UZN \#6 & 8.49 & 39 & USW UZ-N37 & 7.16 \\
\hline 7 & UE-25 UZN \#7 & 8.61 & 40 & USW UZ-N40 & 8.06 \\
\hline 8 & UE-25 UZN \#8 & 8.59 & 41 & USW UZ-N41 & 8.43 \\
\hline 9 & UE-25 UZN \#9 & 8.51 & 42 & USW UZ-N42 & 8.42 \\
\hline 10 & UE-25 UZN \#10 & 8.49 & 43 & USW UZ-N43 & 8.35 \\
\hline 11 & UE-25 UZN \#12 & 8.53 & 45 & USW UZ-N45 & 8.34 \\
\hline 12 & UE-25 UZN \#13 & 7.48 & 46 & USW UZ-N46 & 10.34 \\
\hline 15 & UE-25 UZN \#19 & 8.50 & 47 & USW UZ-N47 & 9.45 \\
\hline 16 & UE-25 UZN \#20 & 8.41 & 48 & USW UZ-N48 & 8.77 \\
\hline 17 & UE-25 UZN \#21 & 8.27 & 49 & USW UZ-N49 & 8.38 \\
\hline 18 & UE-25 UZN \#22 & 8.22 & 50 & USW UZ-N50 & 8.38 \\
\hline 19 & UE-25 UZN \#23 & 8.10 & 51 & USW UZ-N51 & 8.45 \\
\hline 20 & UE-25 UZN \#28 & 8.27 & 52 & USW UZ-N52 & 8.34 \\
\hline 21 & UE-25 UZN \#29 & 8.06 & 55 & USW UZ-N55 & 7.59 \\
\hline 22 & UE-25 UZN \#30 & 8.07 & 56 & USW UZ-N57 & 8.41 \\
\hline 23 & UE-25 UZN \#56 & 8.15 & 58 & USW UZ-N65 & 6.71 \\
\hline 24 & UE-25 UZN \#60 & 7.91 & 59 & USW UZ-N66 & 7.74 \\
\hline 25 & UE-25 UZN \#85 & 7.13 & 60 & USW UZ-N67 & 8.73 \\
\hline 26 & UE-25 UZN \#92 & 7.63 & 61 & USW UZ-N68 & 8.69 \\
\hline 27 & UE-25 UZN \#97 & 8.18 & 63 & USW UZ-N70 & 8.21 \\
\hline 28 & UE-29 UZN \#91 & 9.64 & 64 & USW UZ-N71 & 7.44 \\
\hline 65 & USW UZ-N72 & 7.60 & 85 & USW UZ-N95 & 8.25 \\
\hline 66 & USW UZ-N73 & 8.54 & 87 & USW UZ-N98 & 9.22 \\
\hline 67 & USW UZ-N74 & 7.53 & 88 & USW UZ 13 & 7.63 \\
\hline 68 & USW UZ-N75 & 8.11 & 89 & USW GA-1 & 11.19 \\
\hline 69 & USW UZ-N76 & 8.02 & 91 & UE25 WT-4 & 8.00 \\
\hline 70 & USW UZ-N77 & 8.21 & 93 & USW H-5 & 8.17 \\
\hline 71 & USW UZ-N78 & 8.30 & 94 & $\operatorname{HRF}\left(4^{n}\right)$ & 6.03 \\
\hline 72 & USW UZ-N79 & 9.14 & 95 & USW H-3 & 7.49 \\
\hline 73 & USW UZ-N80 & 8.90 & 98 & Evaporation pan & 5.95 \\
\hline 74 & USW UZ-N81 & 9.16 & 99 & Weather station 4 & 8.22 \\
\hline
\end{tabular}


Table 3. Network precipitation totals for water year 1992--Continued

\begin{tabular}{cccccc}
\hline $\begin{array}{c}\text { Location } \\
\text { code }\end{array}$ & $\begin{array}{c}\text { Site } \\
\text { nomenclature }\end{array}$ & $\begin{array}{c}\text { Total } \\
\text { (inches) }\end{array}$ & $\begin{array}{c}\text { Location } \\
\text { code }\end{array}$ & $\begin{array}{c}\text { Site } \\
\text { nomenclature }\end{array}$ & $\begin{array}{c}\text { Total } \\
\text { (inches) }\end{array}$ \\
\hline 75 & USW UZ-N82 & 8.65 & 100 & G-3 & Weather station 1 \\
76 & USW UZ-N83 & 9.43 & 101 & 7.61 \\
77 & USW UZ-N84 & 8.71 & 103 & Weather station 3 & 8.27 \\
79 & USW UZ-N87 & 8.79 & 105 & Plug Hill & 7.84 \\
80 & USW UZ-N88 & 9.07 & & & \\
& & & & & \\
81 & USW UZ-N89 & 8.83 & & & \\
82 & USW UZ-N90 & 8.69 & & & \\
82 & USW UZ-N90(4") & 8.01 & & & \\
83 & USW UZ-N93 & 8.31 & & & \\
84 & USW UZ-N94 & 8.47 & & & \\
\hline
\end{tabular}


Table 4. Network precipitation totals for water year 1993

[Includes only sites that have a complete annual total]

\begin{tabular}{|c|c|c|c|c|c|}
\hline $\begin{array}{l}\text { Location } \\
\text { code }\end{array}$ & $\begin{array}{c}\text { Site } \\
\text { nomenclature }\end{array}$ & $\begin{array}{c}\text { Total } \\
\text { (inches) }\end{array}$ & $\begin{array}{l}\text { Location } \\
\text { code }\end{array}$ & Site nomenclature & $\begin{array}{c}\text { Total } \\
\text { (Inches) }\end{array}$ \\
\hline 1 & UE-25 UZN \#1 & 11.91 & 29 & USW UZ-N11 & 8.66 \\
\hline 2 & UE-25 UZN \#2 & 11.84 & 29 & USW UZ-N11(4") & 8.63 \\
\hline 3 & UE-25 UZN \#3 & 11.23 & 30 & USW UZ-N15 & 10.18 \\
\hline 4 & UE-25 UZN \#4 & 12.09 & 31 & USW UZ-N16 & 10.22 \\
\hline 5 & UE-25 UZN \#5 & 11.18 & 32 & USW UZ-N17 & 9.97 \\
\hline 6 & UE-25 UZN \#6 & 11.27 & 33 & USW UZ-N24 & 11.60 \\
\hline 7 & UE-25 UZN \#7 & 12.00 & 35 & USW UZ-N25 & 11.58 \\
\hline 8 & UE-25 UZN \#8 & 11.28 & 35 & USW UZ-N26 & 11.26 \\
\hline 9 & UE-25 UZN \#9 & 12.03 & 36 & USW UZ-N27 & 8.75 \\
\hline 10 & UE-25 UZN \#10 & 12.01 & 36 & USW UZ-N27(8") & 9.95 \\
\hline 11 & UE-25 UZN \#12 & 11.44 & 37 & USW UZ-N35 & 11.35 \\
\hline 13 & UE-25 UZN \#14 & 10.92 & 39 & USW UZ-N37 & 11.33 \\
\hline 14 & UE-25 UZN \#18 & 10.98 & 39 & USW UZ-N37(4") & 10.28 \\
\hline 15 & UE-25 UZN \#19 & 11.14 & 40 & USW UZ-N40 & 10.87 \\
\hline 16 & UE-25 UZN \#20 & 11.25 & 41 & USW UZ-N41 & 11.37 \\
\hline 17 & UE-25 UZN \#21 & 11.09 & 42 & USW UZ-N42 & 11.20 \\
\hline 18 & UE-25 UZN \#22 & 11.56 & 43 & USW UZ-N43 & 11.38 \\
\hline 19 & UE-25 UZN \#23 & 11.05 & 44 & USW UZ-N44 & 11.62 \\
\hline 20 & UE-25 UZN \#28 & 11.14 & 45 & USW UZ-N45 & 11.63 \\
\hline 21 & UE-25 UZN \#29 & 10.98 & 46 & USW UZ-N46 & 10.21 \\
\hline 22 & UE-25 UZN \#30 & 11.62 & 47 & USW UZ-N47 & 10.97 \\
\hline 23 & UE-25 UZN \#56 & 11.00 & 48 & USW UZ-N48 & 11.51 \\
\hline 24 & UE-25 UZN \#60 & 11.27 & 49 & USW UZ-N49 & 10.84 \\
\hline 25 & UE-25 UZN \#85 & 9.79 & 50 & USW UZ-N50 & 10.80 \\
\hline 27 & UE-25 UZN \#97 & 11.24 & 51 & USW UZ-N51 & 11.15 \\
\hline 52 & USW UZ-N52 & 10.70 & 82 & USW UZ-N90 & 9.87 \\
\hline 53 & USW UZ-N53 & 10.84 & 82 & USW UZ-N90(4") & 10.83 \\
\hline 56 & USW UZ-N57 & 11.45 & 83 & USW UZ-N93 & 9.01 \\
\hline 60 & USW UZ-N67 & 10.44 & 85 & USW UZ-N95 & 9.03 \\
\hline 60 & USW UZ-N67(4") & 10.28 & 86 & USW UZ-N96 & 8.35 \\
\hline 62 & USW UZ-N69 & 10.59 & 87 & USW UZ-N98 & 11.72 \\
\hline 63 & USW UZ-N70 & 10.48 & 88 & USW UZ 13 & 7.99 \\
\hline 64 & USW UZ-N71 & 7.21 & 93 & USW H-5 & 6.54 \\
\hline 65 & USW UZ-N72 & 6.94 & 94 & HRF Wedge & 9.38 \\
\hline 66 & USW UZ-N73 & 7.69 & 94 & HRF (4") & 10.15 \\
\hline
\end{tabular}


Table 4. Network precipitation totals for water year 1993--Continued

\begin{tabular}{clcclc}
\hline $\begin{array}{c}\text { Location } \\
\text { code }\end{array}$ & $\begin{array}{c}\text { Site } \\
\text { nomenclature }\end{array}$ & $\begin{array}{c}\text { Total } \\
\text { (inches) }\end{array}$ & $\begin{array}{c}\text { Location } \\
\text { code }\end{array}$ & Site nomenclature & $\begin{array}{c}\text { Total } \\
\text { (inches) }\end{array}$ \\
\hline 67 & USW UZ-N74 & 6.97 & 94 & HRF (8") & 9.87 \\
68 & USW UZ-N75 & 7.67 & 95 & USW H-3 & 6.62 \\
69 & USW UZ-N76 & 8.23 & 96 & Weather station 4 (old) & 7.38 \\
70 & USW UZ-N77 & 10.26 & 98 & Evaporation pan & 9.14 \\
71 & USW UZ-N78 & 10.23 & 100 & G-3 & 9.00 \\
72 & USW UZ-N79 & 10.98 & 100 & G-3 (8") & 9.58 \\
73 & USW UZ-N80 & 10.71 & 101 & Weather station 1(8") & 11.18 \\
73 & USW UZ-N80(4") & 11.68 & 102 & Weather station 2 & 9.35 \\
74 & USW UZ-N81 & 10.24 & 103 & Weather station 3 & 10.57 \\
75 & USW UZ-N82 & 9.75 & 103 & Weather station 3(8") & 11.66 \\
77 & & & & & 9.24 \\
78 & USW UZ-N84 & 9.81 & 104 & Fran Ridge & 9.83 \\
79 & USW UZ-N86 & 10.78 & 104 & Fran Ridge (8") & 8.16 \\
80 & USW UZ-N87 & 9.93 & 105 & Plug Hill & 8.32 \\
81 & USW UZ-N88 & 10.42 & 106 & 4JA & 11.50 \\
\hline
\end{tabular}

